\title{
FLIP THEOREM AND THE EXISTENCE OF MINIMAL MODELS FOR 3-FOLDS
}

\author{
SHIGEFUMI MORI \\ To Professor M. Nagata on his 60th birthday
}

\begin{tabular}{|c|c|}
\hline$\S 0$. & Introduction \\
\hline$\S 1$. & Preliminaries and basic definitions \\
\hline $1 \mathrm{a}$ & (Appendix 1a). Results on 3-fold terminal singularities \\
\hline $1 \mathrm{~b}$ & (Appendix 1b). Deformation of extremal nbds \\
\hline$\S 2$. & Numerical invariants $i_{P}(n), w_{P}(0)$, and $w_{P}^{*}(n)$ \\
\hline 3. & Embedding dimension of $\left(C^{\sharp}, P^{\sharp}\right)$ \\
\hline 4. & Classification of $X \supset C$ at $P$ into cases \\
\hline 5. & Numerical calculations for $(\mathrm{IA}),(\mathrm{IC}),\left(\mathrm{IA}^{\vee}\right)$, and $\left(\mathrm{IC}^{\vee}\right)$ \\
\hline 6. & Possible singularities on an extremal nbd $X \supset C \simeq \mathbb{P}^{1}$ \\
\hline$\S 7$. & Existence of "good" anti(bi)canonical divisor (easy case) \\
\hline$\S 8$. & $J$-filtration and lifting structure \\
\hline$\S 9$. & Existence of "good" anticanonical divisor (delicate case) \\
\hline$\S 10$. & A slight generalization of $(0.4 .5)$ and comments on (1a.7) \\
\hline \multicolumn{2}{|c|}{ Appendix A. Summary of local classification of $X \supset C \ni P$} \\
\hline \multicolumn{2}{|c|}{ Appendix B. Possible singularities on an extremal nbd } \\
\hline \multicolumn{2}{|c|}{ List of notation and terminology } \\
\hline & \\
\hline
\end{tabular}

\section{INTRODUCTION}

One of the main achievements of the 19th century Italian school of algebraic geometry was a complete understanding of the birational geometry of surfaces, including the construction of minimal models. During the past several years, a program has emerged to construct minimal models in higher dimensions using something called extremal rays. This paper completes the final step of that Minimal Model Program (MMP) in dimension 3 (cf. an excellent introduction [Ko2] for nonexperts).

We should mention that, even in dimension 3 , there are several things yet to be done in classification theory (e.g. [R3, §4]).

Before formulating our main theorem $(0.2 .5)$, let us explain some corollaries and the background.

We will work over the field $\mathbb{C}$ of complex numbers.

Received by the editors January 29, 1987.

1980 Mathematics Subject Classification (1985 Revision). Primary 14E30, 14E05, 14 E35. 
(0.1) Corollaries to Minimal Model Program for 3-folds. Here are three important corollaries indicating that the canonical divisor $K_{X}$ of a 3-fold $X$ has decisive information.

(0.1.1) Theorem. For every nonsingular projective 3-fold $X$, the graded canonical ring $\bigoplus_{\nu \geq 0} H^{0}\left(X, \mathscr{O}\left(\nu K_{X}\right)\right)$ is finitely generated.

This follows from MMP by virtue of the works of Fujita [Ft], Benveniste [B1] and Kawamata [Kal], and it is considered to be a necessary step in the construction of moduli of 3-folds.

(0.1.2) Theorem. A nonsingular projective 3-fold $X$ is uniruled if and only if $H^{0}\left(X, \mathscr{O}\left(\nu K_{X}\right)\right)=0$ for all $\nu>0$, where an $n$-fold $Z$ is uniruled if there is $a$ dominating rational map $\mathbb{P}^{1} \times Y \rightarrow Z$ for some $(n-1)$-fold $Y$.

This follows from MMP by virtue of the works of Miyaoka [My1, My2, My3], and Miyaoka and Mori [MM]. Indeed some model $X^{\prime}$ birational to $X$ is shown to have a finer structure (cf. (0.3)).

(0.1.3) Theorem. Every birational morphism $f: X \rightarrow Y$ between nonsingular projective 3-folds is a composition of divisorial contractions and directed flips $(c f .(0.2))$.

This gives (in principle) a factorization of birational morphisms of 3-folds with mild singularities (cf. (0.3.11)), and generalizes the first half of Danilov's factorization [Da] of toric birational morphisms of 3-folds to arbitrary birational morphisms.

We should point out that the development of the theory of extremal rays was originally motivated by Hironaka's approach $[\mathrm{H}]$ to the factorization problem using "cones" (although the techniques were based on Kleiman's criterion of ampleness [Kl]).

Let us look at the Minimal Model Conjecture more closely.

(0.2) The Minimal Model Conjecture. We will review the necessary material only briefly and more detailed treatment is given in [KMM, Mr4, Wi].

(0.2.1) Definition. We say that a Weil divisor $D$ on a normal variety $X$ is $\mathbb{Q}$-Cartier if $\nu D$ is Cartier for some $\nu>0$. We note that $X$ with only canonical or terminal singularities has $\mathbb{Q}$-Cartier $K_{X}$, leaving the technical definition to (1.0). We say that $X$ has only $\mathbb{Q}$-factorial singularities if every Weil divisor is Q-Cartier.

Let $X$ be a normal projective variety. Let $\rho(X)$ denote the Picard number $(\in \mathbb{N})$, the rank of the Neron-Severi group $\mathrm{NS}(X)$ of $X$. We say that $X$ is a canonical (resp. minimal) model if $X$ has only canonical (resp. terminal) singularities and $K_{X}$ is ample (resp. nef), where a $\mathbb{Q}$-Cartier divisor $H$ is nef iff $(H \cdot C) \geq 0$ for every irreducible curve $C$. 
We say that $X$ has a canonical (resp. minimal) model if there is a canonical (resp. minimal) model birational to $X$. We remark that such a canonical model is unique while a minimal model is not.

(0.2.2) MMC, in short, claims that every variety $X$ has a minimal model iff $X$ is not uniruled. To make it more explicit, we will review the theory of extremal rays built by Benveniste, Kawamata, Kollár, Mori, Reid, Shokurov (cf. [KMM, Mr4, R3, or Wi]. To simplify the exposition, we treat the core of the theory as a black box; it implies the following.

(0.2.3) Theorem. Let $X$ be a projective variety with only $\mathbb{Q}$-factorial terminal singularities. If $X$ is not a minimal model, then there is a surjective morphism $f: X \rightarrow Y$ to a normal projective variety $Y$ with connected fibers such that $\rho(X)=\rho(Y)+1,-K_{X}$ is $f$-ample and one of the following holds.

(a) $\operatorname{dim} X>\operatorname{dim} Y(\mathbb{Q}$-Fano fibering).

(b) $f$ is birational and contracts a divisor (divisorial contraction).

(c) $f$ is birational and contracts no divisors (small contraction).

(0.2.4) Let the notation and the assumptions be as in (0.2.3). If $f$ is a divisorial contraction, it is easy to see that $Y$ has only $\mathbb{Q}$-factorial terminal singularities. The case of a small contraction is treated by our main theorem if $X$ is a 3-fold.

(0.2.5) Main Theorem (Flip Theorem). Let $f: X \rightarrow Y$ be a birational morphism between normal projective 3-folds such that $X$ has only $\mathbb{Q}$-factorial terminal singularities, $\rho(X)=\rho(Y)+1,-K_{X}$ is $f$-ample and $f$ contracts no divisors (to curves or points). Then there is a birational morphism $f^{\prime}: X^{\prime} \rightarrow Y$ from a projective 3-fold $X^{\prime}$ with only $\mathbb{Q}$-factorial terminal singularities such that $f^{\prime}$ contracts no divisors and $K_{X^{\prime}}$ is $f^{\prime}$-ample. (Let us emphasize the change in the sign of the canonical divisor. )

The map $X \rightarrow X^{\prime}$ is isomorphic in codimension 1 (i.e. induces $X-Z \simeq$ $X^{\prime}-Z^{\prime}$ for some closed subsets $Z$ and $Z^{\prime}$ of codim $\left.\geq 2\right), \rho(X)=\rho\left(X^{\prime}\right)$, and $f^{\prime}$ (or $X^{\prime}$ ) is called the directed flip (or flip) of $f$. If $\operatorname{dim} X \geq 4$, the existence of the directed flip is unknown.

There are a couple of ways to put (0.2.3)-(0.2.5) together to state the Minimal Model Program. One of the formulations is the following. We note that (c) is conjectural if $\operatorname{dim} X \geq 4$.

(0.2.6) Minimal Model Program (Reid [R2]: an observation based on [Mr2] and Francia [Fr]). Let $X$ be a projective 3-fold with only $\mathbb{Q}$-factorial terminal singularities. If $X$ is not a minimal model, then there is a morphism $f: X \rightarrow Y$ which is a $\mathbb{Q}$-Fano fibering, a divisorial contraction or a small contraction. We treat three cases.

Case (a). No divisorial or small contractions exist: $f$ is a $\mathbb{Q}$-Fano fibering.

Case (b). $f$ is a divisorial contraction: Since $Y$ has only $\mathbb{Q}$-factorial terminal singularities $(0.2 .4)$, we may continue the program by replacing $X$ with $Y$. We note $\rho(Y)=\rho(X)-1$. 
Case (c). $f$ is a small contraction: Since the directed flip $X^{\prime}$ of $X \rightarrow Y$ has only $\mathbb{Q}$-factorial terminal singularities $(0.2 .5)$, we may continue by replacing $X$ with $X^{\prime}$. We note $\rho\left(X^{\prime}\right)=\rho(X)$.

Since $\rho(X)$ is a natural number, (b) does not occur infinitely many times, and the following theorem of Shokurov shows that (c) does not repeat infinitely many times either.

(0.2.7) Theorem (Shokurov [Sh]). To each algebraic 3-fold with only terminal singularities is associated a nonnegative integer $d(X)$ called the difficulty, and one has $d\left(X^{\prime}\right)<d(X)$ for $X$ and $X^{\prime}$ as in $(0.2 .6(\mathrm{c}))$.

Though the existence of flip is unknown if $\operatorname{dim} X \geq 4$, the termination of flips (0.2.7) is generalized to 4-folds $X$ by Kawamata, Matsuda, and Matsuki [KMM].

These can be put together into the flow chart for 3-folds.

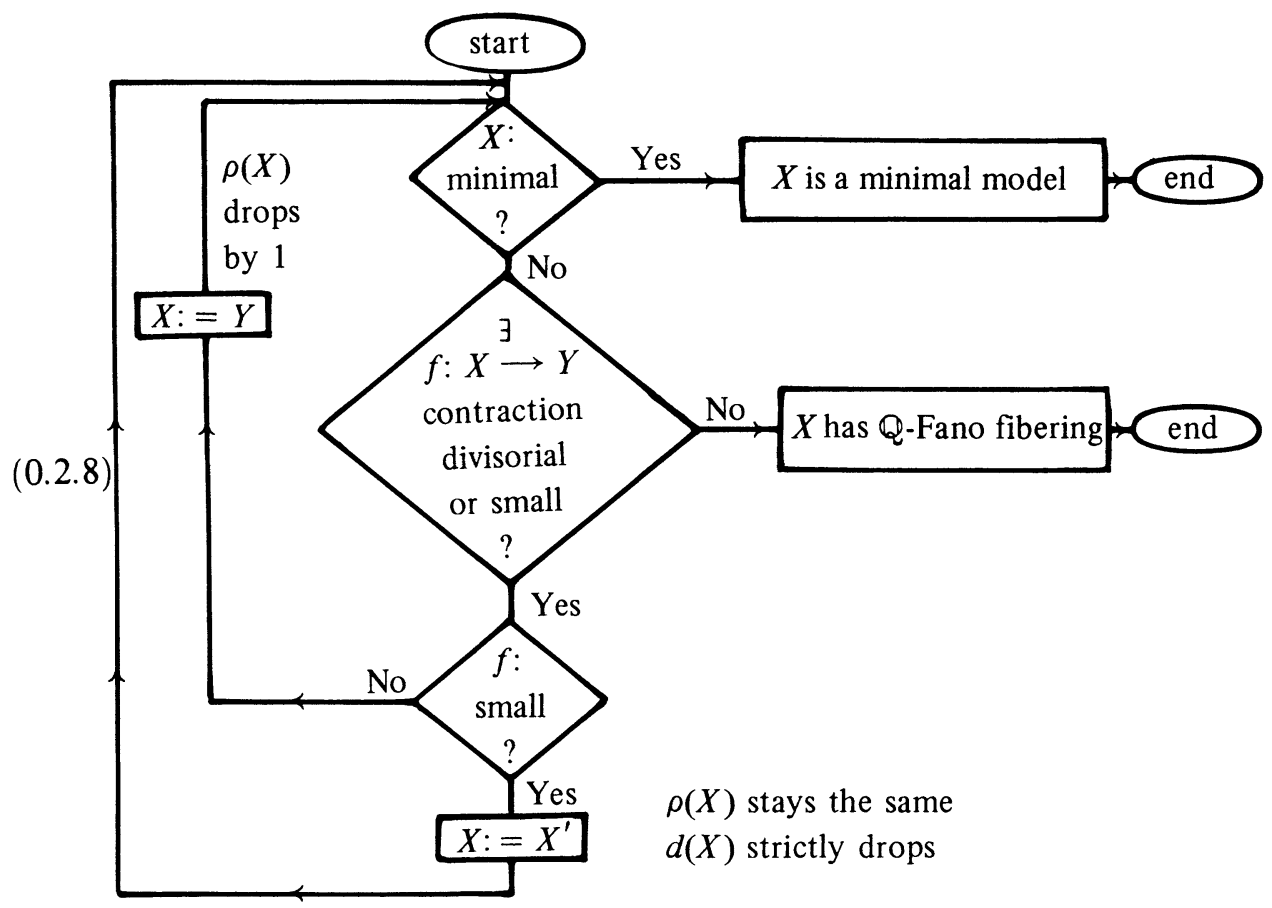

We will show how the results in (0.1) follow from the Minimal Model Program for 3-folds and state some more corollaries.

(0.3) Results in (0.1) and further corollaries. The Minimal Model Program is thus completed for 3 -folds and

(0.3.1) Theorem. Every 3-fold has a minimal model or a model with a $\mathbb{Q}$-Fano fibering. 
We recall that a nonsingular projective variety $X$ is of general type if the rational mapping associated to $H^{0}\left(X, \mathscr{O}\left(\nu K_{X}\right)\right)$ is birational for some $\nu>0$, and that a $\mathbb{Q}$-Cartier divisor $D$ is semiample if $\nu D$ is a Cartier divisor such that $\mathscr{O}(\nu D)$ is generated by global sections for some $\nu>0$.

We quote several results.

(0.3.2) Theorem (Kawamata [Ka1, KMM] and Benveniste [B1]). Let $X$ be a nonsingular projective variety of general type which has a minimal model (say, $Y)$. Then $K_{Y}$ is semiample, and hence $X$ has a canonical model and the canonical ring of $X$ is finitely generated.

This implies $(0.1 .1)$, since [Ft] settles $(0.1 .1)$ when $X$ is a 3 -fold which is not of general type. We will explain (0.1.2).

(0.3.3) Theorem (Miyaoka [My1, My2, My3]). If a nonsingular projective 3-fold $X$ has a minimal model, then $H^{0}\left(X, \mathscr{O}\left(\nu K_{X}\right)\right) \neq 0$ for some $\nu>0$.

We note that the generalization of $(0.3 .3)$ to the case of $\operatorname{dim} X \geq 4$ is unknown.

Thus if we start from a nonsingular projective 3-fold $X$ such that

$$
H^{0}\left(X, \mathscr{O}\left(\nu K_{X}\right)\right)=0 \text { for all } \nu>0
$$

then we never get a minimal model and hence we get a model $X^{\prime}$ with a $\mathbb{Q}$-Fano fibering. Then we apply the following to get (0.1.2).

(0.3.4) Theorem (Miyaoka and Mori [MM]). If $f: X \rightarrow Y$ is a $\mathbb{Q}$-Fano fibering, then $X$ is uniruled.

Most of the above results in the absolute cases have been generalized to the relative case based on Kollár's vanishing theorem [Kol]. For instance, (0.3.2) is a special case of the following (0.3.5), which is also a special case of Kawamata's relative Base Point Free Theorem.

(0.3.5) Theorem (Kawamata [KMM, Theorem 3-3-1]). Let $f: X \rightarrow S$ be a projective morphism with connected fibers from a variety $X$ with only terminal singularities to a variety $S$. If a general fiber of $f$ is a variety of general type and $K_{X}$ is $f$-nef, then $K_{X}$ is $f$-semiample.

(0.3.6) Remark. In the above, a divisor $D$ on $X$ is $f$-nef if $(D \cdot C) \geq 0$ for all irreducible curves $C$ on $X$ such that $f(C)$ is a point, and it is $f$-semiample if the natural map $f^{*} f_{*} \mathscr{O}(n D) \rightarrow \mathscr{O}(n D)$ is surjective for sufficiently divisible $n>0$.

Unlike the absolute case, there are sometimes only trivial minimal models in the relative case. 
(0.3.7) Proposition. Let $g: Z \rightarrow X$ be a birational projective morphism of normal varieties such that $K_{Z}$ is $\mathbb{Q}$-Cartier and $X$ has only $\mathbb{Q}$-factorial terminal singularities. If $g$ is not an isomorphism, then $K_{Z}$ is not $g$-nef.

(0.3.8) Remark. This is proved for smooth projective 3-folds $X$ and $Z$ in [Mr2, (3.40)], whose proof works for (0.3.7) modulo trivial changes.

(0.3.9) The Minimal Model Program works equally well for 3-folds with $\mathbb{Q}$-factorial terminal singularities which are projective over a fixed irreducible reduced algebraic variety $S$ as mentioned after $(0.3 .4)$ because $(0.2 .5)$ is proved in a more general form (0.4.1). We state a theorem which is an immediate corollary to the Minimal Model Program for 3-folds in the relative setting.

(0.3.10) Theorem. Let $f: X \rightarrow S$ be a projective morphism from a 3-fold $X$ with only $\mathbb{Q}$-factorial terminal singularities to a variety $S$. Then via a composition of divisorial contractions and directed flips, $X$ is $S$-birational to an $S$-projective 3-fold $f^{\prime}: X^{\prime} \rightarrow S$ such that $X^{\prime}$ has only $\mathbb{Q}$-factorial terminal singularities and satisfies one of the following.

(a) $K_{X^{\prime}}$ is $f^{\prime}$-nef, or

(b) $X^{\prime}$ has a surjective $S$-morphism to an $S$-projective variety $Z \rightarrow S$ such that $\operatorname{dim} Z<\operatorname{dim} X,-K_{X^{\prime}}$ is relatively ample over $Z$, and the relative Picard number $\rho\left(X^{\prime} / Z\right)$ of $X^{\prime}$ over $Z$ is 1 .

(0.3.10.1) Remark. In the important special case where $f$ is a semistable projective morphism onto a smooth curve, we note (i) "minimal" models (maybe nonalgebraic) over $S$ were constructed by Kulikov [Ku] and Persson and Pinkham [PP] if the general fibers of $f$ are surfaces with $K \sim 0$ (the first breakthrough in this direction) and by Morrison [Mrrl] if the general fibers of $f$ are surfaces with $2 K \sim 0$ and $K \not 0$ (they did not use extremal rays), (ii) Tsunoda [Mn, T], Shokurov and Mori independently proved (0.3.10) if the general fibers of $f$ are minimal surfaces, and (iii) Kawamata [Ka2] proved (0.3.10) with no assumptions on the general fibers.

By virtue of (0.3.7), the following holds.

(0.3.11) Theorem. Every birational projective morphism $f: X \rightarrow S$ between 3-folds with only $\mathbb{Q}$-factorial terminal singularities is a product of divisorial contractions and directed flips.

We note that $(0.1 .3)$ is only a special case of $(0.3 .11)$.

By (0.3.5) quoted earlier, we obtain

(0.3.12) Theorem. Let $S$ be a 3-fold. Then there exist projective birational morphisms $f: X \rightarrow S$ and $g: Y \rightarrow S$ such that

(i) $X$ has only $\mathbb{Q}$-factorial terminal singularities and $K_{X}$ is $f$-semiample, and

(ii) $Y$ has only canonical singularities, $K_{Y}$ is $f$-ample, and there is an $S$ morphism $h: X \rightarrow Y$ such that $K_{X}=h^{*} K_{Y}$. 
We note that such $Y$ is unique while such $X$ is unique only in codimension 1 , and we call $X$ a $\mathbb{Q}$-factorial terminal modification and $Y$ the canonical modification of $S$.

These results have two kinds of rather technical generalizations.

(0.3.13) Remark. When $S$ is the germ $(S, s)$ of an analytic space at a point $s$ or a compact analytic space, the results corresponding to $(0.3 .10)-(0.3 .12)$ hold by Nakayama $[N]$ because $(0.2 .5)$ is proved in the analytic space case $(0.4 .1)$, where an analytic space $Z$ over $S$ is said to have only $\mathbb{Q}$-factorial singularities if every global Weil divisor on $Z$ is $\mathbb{Q}$-Cartier.

(0.3.14) Remark. By virtue of the definition of the extremal ray, the results in (0.2) extend to $G$-threefolds in Manin's sense [Ma] (hence to 3-folds over any field $k$ of characteristic 0 ) as indicated by [Mr2, Chapter 2, §3]. Let us take a finite group $G$ for simplicity. The results in $(0.2)$ hold true if we interpret varieties (resp. subvarieties, morphisms) as varieties with regular $G$-action (resp. $G$-stable subvarieties, $G$-equivariant morphisms). Here, $G \mathbb{Q}$-factoriality (the modified $\mathbb{Q}$-factoriality) means that all the $G$-stable Weil divisors are $\mathbb{Q}$-Cartier, and $G \rho(X)$ (the modified $\rho(X))$ denotes the rank of the $G$-invariant part of $\operatorname{NS}(X)$. This way, $(0.3 .1),(0.3 .10),(0.3 .11)$, and $(0.3 .12)$ hold true for $G$-threefolds.

Let us refine (0.3.1) (cf. [I, Ma, and Ko3, (3.6)]), where $p G 3 f t$ is an abbreviation of "projective $G$-threefold with only $G \mathbb{Q}$-factorial terminal singularities."

(0.3.14.1) Theorem. Any $p G 3 f t$ is G-birational to either

(i) a pG3ft $X$ with nef $K_{X}$ (G-minimal model ), or

(ii) a pG3ft $X$ with a surjective $G$-morphism $f$ to a normal projective $G$-variety $Y$ with connected fibers such that $G \rho(X)=G \rho(Y)+1,-K_{X}$ is $f$-ample and $\operatorname{dim} Y<\operatorname{dim} X \quad(G \mathbb{Q}$-Fano fibering $)$.

Let us explain what is done in this paper to prove $(0.2 .5)$.

(0.4) What is to be done to prove the Flip Theorem. Let us modify $(0.2 .5)$ to a statement which is formulated in both algebraic and analytic contexts without Q-factoriality.

(0.4.1) Flip Theorem. Let $f: X \rightarrow Y$ be a proper birational morphism of normal algebraic (resp. analytic) 3-folds such that $X$ has only terminal singularities, $f$ contracts no divisors, and $-K_{X}$ is $f$-ample. Then there is a proper birational morphism $f^{\prime}: X^{\prime} \rightarrow Y$ from an algebraic (resp. analytic) 3-fold $X^{\prime}$ with only terminal singularities such that $f^{\prime}$ contracts no divisors and $K_{X^{\prime}}$ is $f^{\prime}$-ample.

We note that $X^{\prime}$ is unique and its existence is equivalent to the finite generation of $\bigoplus_{n \geq 0} f_{*} \mathscr{O}\left(n K_{X}\right)$ as an $\mathscr{O}_{X}$-algebra. The birational map $X \rightarrow X^{\prime}$ is isomorphic in codimension 1 and $f^{\prime}$ (or $X^{\prime}$ ) is called the directed flip (or flip) of $f$. 
(0.4.1.1) Remark. (i) The conclusion of (0.4.1) holds even if $X$ has canonical singularities. This can be proved by the arguments as in [Ka2, Ko3], or by applying the Minimal Model Program over $Y$, which are both based on the original (0.4.1), and

(ii) the two versions of $(0.4 .1)$, the algebraic $(0.4 .1)_{\mathrm{alg}}$ and the analytic $(0.4 .1)_{\text {an }}$, are equivalent as in [Ka2, Proposition 8.4]. It is based on the rationality of the singularities of $Y$ and Shokurov's termination of directed flips (0.2.7).

$(0.4 .2)$ It is easy to see $(0.4 .1)_{\mathrm{alg}} \Rightarrow(0.2 .5)$, and $(0.4 .1)_{\text {an }}$ is to be proved. The advantage is the following: The fundamental set $\Sigma$ on $Y$ for $f^{-1}$ is discrete and we may set $X^{\prime}-f^{\prime^{-1}} \Sigma=Y-\Sigma$. Thus the problem is local at each point of $\Sigma$ and we may assume $Y$ is a germ $(Y, y)$ at a point. For $f: X \rightarrow(Y, y)$ as in (0.4.1), we call the germ $X \supset f^{-1}(y)_{\text {red }}$ of an analytic 3-fold $X$ along $f^{-1}(y)_{\text {red }}$ an extremal nbd (as in (1.1)).

By treating irreducible components of $f^{-1}(y)$ one by one [Ka2, Proposition 8.4 , one can reduce $(0.4 .1)_{\text {an }}$ to

(0.4.3) Lemma. $(0.4 .1)_{\text {an }}$ holds if we assume further that $Y$ is the germ $(Y, y)$ at a point $y$ and $f^{-1}(y)$ is irreducible.

We should mention that $f^{-1}(y) \simeq \mathbb{P}^{1}$ above. This follows from the rationality of the singularity of $(Y, y)(1.3)$.

Kawamata's approach [Ka2] to the directed flip is as follows:

(0.4.4) Take a double cover $V$ of $X$ with branch locus $D \in\left|-2 K_{X}\right|$. If $V$ has only canonical singularities then so does the double cover $W$ of $Y$ with branch locus $f(D)$ and one can use finite generation of $\bigoplus_{n \geq 0} \mathscr{O}_{W}\left(n K_{Y}\right)[\mathrm{Ka} 2$, Theorem 6.1'] (a simpler proof is given in [Ko3]) to get finite generation of its $\mathbb{Z}_{2}$-invariant part $\bigoplus_{n \geq 0} \mathscr{O}_{Y}\left(n K_{Y}\right)=\bigoplus_{n \geq 0} f_{*} \mathscr{O}_{X}\left(n K_{X}\right)$.

Thus [Ka2, Proposition 8.7] reduces (0.4.3) to the following.

(0.4.5) Theorem. Let $X \supset C \simeq \mathbb{P}^{1}$ be an extremal nbd. Then one of the following on the linear system $\left|-a K_{X}\right|(a=1$ or 2$)$ holds.

(i) $\left|-K_{X}\right|$ has a member $D$ with only rational double points, or

(ii) $\left|-2 K_{X}\right|$ has a member $D$ so that the double cover $Z$ of $X$ with branch locus $D$ has only canonical singularities.

Kawamata [Ka2] proved (0.4.5) in the case of 1-parameter semistable family of surfaces to prove the result mentioned in (0.3.10.1(iii)).

It is (0.4.5) that is actually proved in this paper (Theorems (7.3) and (9.10), cf. also Appendix B). The main information we use for the extremal nbd $X \supset$ $C \simeq \mathbb{P}^{1}$ is

$$
H^{1}\left(\mathscr{O}_{Z}\right)=H^{1}\left(\omega_{X} \otimes \mathscr{O}_{Z}\right)=0 \forall Z \subset X \text { with } Z_{\text {red }}=C
$$


These conditions are similar to those in [Mr2, §9] (cf. (1.2.1), §10), and so are some of our arguments.

The following is the smooth case of (0.4.5). We will illustrate our approach by proving $(0.4 .7)$ via $(0.4 .6)$. We should emphasize that we are interested in the arguments not the results. The results show that the smooth $X$ is the blowup of a smooth 3-fold $(Y, Q)$ along a smooth curve $(Z, Q)$ and $C$ is the fiber over $Q$, which means that extremal nbds must have singular points (cf. $\S 10)$.

(0.4.7) Proposition. Let $X \supset C \simeq \mathbb{P}^{1}$ be a smooth extremal nbd. Then $\mathscr{O}_{C}\left(K_{X}\right)$ $\simeq \mathscr{O}_{C}(-1), I_{C} / I_{C}{ }^{2} \simeq \mathscr{O}_{C} \oplus \mathscr{O}_{C}(1)$, and $\left|-K_{X}\right|$ has a smooth member, where $I_{C}$ is the ideal sheaf of $C$ in $X$.

(0.4.8) Proof. We see $\left(K_{X} \cdot C\right)=-1$ from $\left(K_{X} \cdot C\right)<0$ and $H^{1}\left(\mathscr{O}_{C}\left(K_{X}\right)\right)=0$ (0.4.6). By the standard exact sequence

$$
0 \rightarrow I_{C} / I_{C}^{2} \rightarrow \Omega_{X}^{1} \otimes \mathscr{O}_{C} \rightarrow \mathscr{O}\left(K_{C}\right) \rightarrow 0,
$$

one has a natural isomorphism

$$
\alpha_{1}: \bigwedge^{2}\left(I_{C} / I_{C}^{2}\right) \stackrel{\sim}{\longrightarrow} \mathscr{O}\left(K_{X}\right) \otimes \mathscr{O}_{C}\left(-K_{C}\right) \text {, }
$$

and $\operatorname{deg} I_{C} / I_{C}{ }^{2}=\left(K_{X} \cdot C\right)-\operatorname{deg} K_{C}=1$. By the exact sequence

$$
0 \rightarrow I_{C} / I_{C}^{2} \otimes \mathscr{O}_{C}(-1) \rightarrow \mathscr{O}_{X} / I_{C}{ }^{2} \otimes \mathscr{O}_{X}\left(K_{X}\right) \rightarrow \mathscr{O}_{C}(-1) \rightarrow 0
$$

and (0.4.6), we see $H^{1}\left(I_{C} / I_{C}{ }^{2} \otimes \mathscr{O}_{C}(-1)\right)=0$. Whence $I_{C} / I_{C}{ }^{2} \otimes \mathscr{O}_{C}(-1) \simeq$ $\mathscr{O}_{C} \oplus \mathscr{O}_{C}(-1)$ since it has degree $1-2=-1$. Hence follows the second assertion. Let $x \in C$. Let $(D, x)$ be a smooth divisor in the germ $(X, x)$ intersecting transversally with $(C, x)$, then $(D, x)$ extends to a divisor $D^{\prime}$ of $X$ since $X$ is the germ along the curve $C$. Then $\left(D^{\prime} \cdot C\right)=1=\left(-K_{X} \cdot C\right)$. Hence $D^{\prime} \in\left|-K_{X}\right|$ since Pic $X \ni L \mapsto(L \cdot C) \in \mathbb{Z}$ induces $\operatorname{Pic} X \simeq \mathbb{Z}$ by (0.4.6) (cf. $(0.1 .3))$.

(0.4.9) The arguments for singular extremal nbds $X \supset C \simeq \mathbb{P}^{1}$ are more elaborate. We will indicate how the arguments in (0.4.8) are modified to obtain results similar to (0.4.7).

We start with easy definitions.

(0.4.9.1) Definition. For a coherent sheaf $\mathscr{F}$, let $\operatorname{gr}_{C}^{0}(\mathscr{F})=\mathscr{F} \otimes \mathscr{O}_{C} / T$, where $T\left(\subset \mathscr{F} \otimes \mathscr{O}_{C}\right)$ is the maximal subsheaf of finite length. The sheaf $\operatorname{gr}_{C}^{0}(\mathscr{F})$ satisfies $\mathscr{F} \otimes \mathscr{O}_{C} \simeq \operatorname{gr}_{C}^{0}(\mathscr{F}) \oplus T$. For $n>0$, let $I_{C}{ }^{(n)}$ be the $n$th symbolic power of $I_{C}$ which is determined by

$$
\mathscr{O}_{X} / I_{C}{ }^{(n)}=\left(\mathscr{O}_{X} / I_{C}{ }^{n}\right) /(\text { maximal subsheaf of finite length }) \text {. }
$$

(0.4.10) Analogues of “ $\omega_{X} \otimes \mathscr{O}_{C} \simeq \mathscr{O}_{C}(-1)$." Let us look at $\operatorname{gr}_{C}^{0} \omega$ $\left(=\operatorname{gr}_{C}^{0}\left(\omega_{X}\right)\right)$. Let $m$ be a positive integer such that $m K_{X}$ is a Cartier divisor. 
Since

induces an injection

$$
\left(\omega_{X} \otimes \mathscr{O}_{C}\right)^{\otimes m} \rightarrow \mathscr{O}_{C}\left(m K_{X}\right)
$$

$$
\beta_{0}:\left(\operatorname{gr}_{C}^{0} \omega\right)^{\otimes m} \hookrightarrow \mathscr{O}_{C}\left(m K_{X}\right)
$$

we see $\operatorname{deg} \operatorname{gr}_{C}^{0} \omega<0$ by $\left(K_{X} \cdot C\right)<0$. Thus we see one analogue

$$
\operatorname{gr}_{C}^{0} \omega \simeq \mathscr{O}_{C}(-1)
$$

by $H^{1}\left(\omega_{X} \otimes \mathscr{O}_{C}\right)=0(0.4 .6)$, and another

$$
\left(K_{X} \cdot C\right)=-1+\sum_{P} w_{P}(0)
$$

by $\beta_{0}: \mathscr{O}(-m) \hookrightarrow \mathscr{O}_{C}\left(m K_{X}\right)$, where $w_{P}(0)=\left(\operatorname{len}_{P}\right.$ Coker $\left.\beta_{0}\right) / m(\geq 0)$ for $P \in C$. In particular, we have

(0.4.11.1) $-1 \leq\left(K_{X} \cdot C\right)<0$ (cf. [B2, Théorème 0]).

(0.4.11) Analogues of “ $I_{C} / I_{C}{ }^{2} \simeq \mathscr{O} \oplus \mathscr{O}(1)$." Let us look at $\operatorname{gr}_{C}^{0} I \quad\left(=\operatorname{gr}_{C}^{0}\left(I_{C}\right)\right)$. (It is equal to $\operatorname{gr}_{C}^{1} \mathscr{O}=I_{C} / I_{C}{ }^{(2)}(2.2)$ in the text.) It is a locally free $\mathscr{O}_{C}$-module of rank 2. Instead of $\alpha_{1}$ in (0.4.8.1), we have a natural injection

$$
\alpha_{1}: \bigwedge^{2} \operatorname{gr}_{C}^{0} I \hookrightarrow \operatorname{gr}_{C}^{0} \omega \otimes \mathscr{O}_{C}\left(-K_{C}\right) \simeq \mathscr{O}(1)
$$

For $P \in C$, let $i_{P}(1)=\operatorname{len}_{P} \operatorname{Coker} \alpha_{1}$ so that

$$
\operatorname{deg} \operatorname{gr}_{C}^{0} I=1-\sum_{P} i_{P}(1)
$$

A local computation shows

(0.4.11.3) Lemma $(=(2.15))$. If $P$ is a singular point of $X$ then $i_{P}(1)>0$.

Our proof for the existence of good members of $\left|-K_{X}\right|$ or $\left|-2 K_{X}\right|$ is based on two kinds of classifications of singularities of $X \supset C$, one local and the other global.

Let us see several results without going into technical details.

(0.4.12) Classification of germs $(X, P) \supset(C, P)$. Using $w_{P}(0)$ and $i_{P}(1)$ and a similar invariant $w_{P}^{*}(1)$, one can give a classification of $(X, P) \supset(C, P)$ without too much trouble (it is done in $\S 3$ and (4.2), cf. Appendix A for results).

Let $P$ be a singular point on an extremal nbd $X \supset C \simeq \mathbb{P}^{1}$. We recall that the index of $P$ is the smallest positive integer $m$ such that $m K_{X}$ is Cartier at $P$. The index $m$ is invariant under deformation and has a topological interpretation:

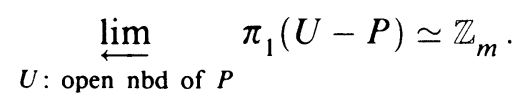

We say that $P$ is ordinary $(4.5)$ if $(X, P)$ is an ordinary double point or a cyclic quotient terminal singularity. The classification has a simple but important 
(0.4.12.1) Corollary. An arbitrary singular point $P$ of $X \supset C$ has an open neighborhood $U$ so that general deformations of the pair $U \supset C \cap U$ have only ordinary points.

Any deformation of the pair $U \supset C \cap U$ can be extended to a deformation of the pair $X \supset C(\S 1 \mathrm{~b}, \S 10)$.

(0.4.12.2) Deformation Lemma. Arbitrary extremal $n b d s \quad X \supset C \simeq \mathbb{P}^{1}$ deform to extremal nbds $X^{\prime} \supset C^{\prime}$ with only ordinary points.

To be rigorous, the lemma is wrong as it is: The deformation $X^{\prime}$ of $X$ often contains finitely many compact curves other than $C^{\prime}$ but it is true that the germ $X^{\prime \circ} \supset C^{\prime}$ of $X^{\prime}$ along $C^{\prime}$ is an extremal nbd. This is, however, a matter of technical details.

(0.4.12.3) Remark. The strategy behind (0.4.12.2) is that, for certain problems on extremal nbds, it is enough to treat extremal nbds with only ordinary points. Though we cannot apply the strategy directly to the flip theorem, we can still apply it to various auxiliary problems (e.g. (0.4.13.1)).

Here is an easy example to use in our introduction.

(0.4.12.4) Example. Let $\boldsymbol{\mu}_{m}$ act on $\left(X^{\sharp}, P^{\sharp}\right)=\left(\mathbb{C}^{3}, 0\right)$ by

$$
\zeta(x, y, z)=\left(\zeta x, \zeta^{a} y, \zeta^{-1} z\right) \quad\left(\zeta \in \mu_{m}\right),
$$

where $a$ is an integer prime to $m$ such that $0<a<m$. Let $C^{\sharp} \subset X^{\sharp}$ be the $x$-axis. Then $(X, P)=\left(X^{\sharp}, P^{\sharp}\right) / \mu_{m} \supset(C, P)=\left(C^{\sharp}, P^{\sharp}\right) / \mu_{m}$ is an example of an ordinary point of index $m$.

We note that any ordinary $(X, P)$ of index $m>1$ is of the above form, but in general $C^{\sharp}$ is singular at $P^{\sharp}$.

The following are easy explicit computations.

(i) $\mathscr{O}_{C, P}=\mathbb{C}\left\{x^{m}\right\}$,

(ii) $\operatorname{gr}_{C}^{0} \omega=\mathscr{O}_{C}\left(x^{m-a} d x \wedge d y \wedge d z\right)$, $\mathscr{O}_{C}\left(m K_{X}\right)=\mathscr{O}_{C}(d x \wedge d y \wedge d z)^{m}$ near $P$,

(iii) $w_{P}(0)=(m-a) / m$,

(iv) $\operatorname{gr}_{C}^{0} I=\mathscr{O}_{C}\left(x^{m-a} y\right) \oplus \mathscr{O}_{C}(x z)$ near $P$, and

(v) $i_{P}(1)=1$.

(0.4.13) Classification of extremal nbds $X \supset C$. This part is done in $\S 6$. Arguments involving $w_{P}(0), i_{P}(1)$ and other higher order numerical invariants prove the following coarse classification of extremal nbds $X \supset C$ (cf. Appendix $B$ for the precise results).

(0.4.13.1) Proposition. Let $X \supset C \simeq \mathbb{P}^{1}$ be an extremal nbd. Then we have

(i) $X$ has at most one singular point with index 1 on $C$,

(ii) $X$ has at most two points with index $>1$ on $C$, and

(iii) if $X$ has three singular points, one of them has index 2 . 
We present two arguments concerning (0.4.13.1).

(0.4.13.2) Here, only to give the idea about our approach, let us prove that $X$ has at most three singular points on $C$. The complex $I_{C} / I_{C}{ }^{2} \rightarrow \mathscr{O}_{X} / I_{C}{ }^{2} \rightarrow$ $\mathscr{\sigma}_{C} \rightarrow 0$ induces an exact sequence

$$
0 \rightarrow \mathrm{gr}_{C}^{0} I \rightarrow \mathscr{O}_{X} / I_{C}^{(2)} \rightarrow \mathscr{O}_{C} \rightarrow 0
$$

Since $H^{0}\left(\mathscr{O}_{X} / I_{C}{ }^{(2)}\right) \rightarrow H^{0}\left(\mathscr{O}_{C}\right)=\mathbb{C}$ is surjective, one has $H^{1}\left(\operatorname{gr}_{C}^{0} I\right)=0$ by $H^{1}\left(\mathscr{O}_{X} / I_{C}{ }^{(2)}\right)=0(0.4 .6)$. Thus $\operatorname{deg} \operatorname{gr}_{C}^{0} I \geq-2$ and it is now immediate to see that $X$ has at most three singular points on $C$ by (0.4.11.2) and (0.4.11.3).

(0.4.13.3) We sketch an easy topological proof of (ii) of (0.4.13.1). We will disprove the case of an extremal nbd $X \supset C \simeq \mathbb{P}^{1}$ with exactly three singular points $P, Q, R$, which are cyclic quotient singular points of indices $a, b, c>$ 1. (The general case can be either reduced to this case by Deformation Lemma (0.4.12.2), or treated in a similar way using a certain nbd $U$ for each $P$ such that $\pi_{1}(U-P) \simeq \mathbb{Z}_{(\text {index } P)}$.)

Here is a homotopical description of $X-\{P, Q, R\}:$ In $C-\{P, Q, R\}=$ $S^{2}-\{P, Q, R\}$, let $\rho$ (resp. $\sigma, \tau$ ) be small loops around $P$ (resp. $Q, R$ ) in $S^{2}$. Embed $\rho, \sigma, \tau$ into lens spaces in certain ways: $\rho \subset S^{5} / \mu_{a}, \sigma \subset S^{5} / \mu_{b}$, $\tau \subset S^{5} / \mu_{c}$. Then $S^{2}-\{P, Q, R\}, S^{5} / \mu_{a}, S^{5} / \mu_{b}, S^{5} / \mu_{c}$ glued together by $\rho, \sigma, \tau$ become homotopically equivalent to $X-\{P, Q, R\}$.

The (class of the) loop $\rho$ need not generate $\pi_{1}\left(S^{5} / \mu_{a}\right)$. But the case $\pi_{1}\left(S^{5} / \mu_{a}\right) \neq\langle\rho\rangle$ (cf. $\left.(0.4 .16)\right)$ can be reduced to the case $\pi_{1}\left(S^{5} / \mu_{a}\right)=\langle\rho\rangle$ in an easy way $((1.13)$ and $(1.16))$. Hence we may assume that $\pi_{1}\left(S^{5} / \mu_{a}\right)=$ $\langle\rho\rangle, \ldots, \pi_{1}\left(S^{5} / \mu_{c}\right)=\langle\tau\rangle$. Thus

$$
\pi_{1}(X-\{P, Q, R\}) \simeq\langle\rho, \sigma, \tau\rangle /\left\{\rho^{a}=1, \sigma^{b}=1, \tau^{c}=1, \rho \sigma \tau=1\right\}
$$

by Van Kampen's Theorem. This group is known to have a finite quotient group in which the image of $\rho$ (resp. $\sigma, \tau$ ) is exactly of order $a$ (resp. $b, c$ ). The associated covering space of $X-\{P, Q, R\}$ is extended to a finite covering of $X$ which is a smooth extremal nbd. This is impossible by the comment preceding (0.4.7).

Based on these, our case-dependent arguments go as follows.

(0.4.14) Local methods to find members $\in\left|-K_{X}\right|,\left|-2 K_{X}\right|$. This part is done in $\S 7$ and generalizes an argument in (0.4.8). For each $P \in C$ of index $>1$ (say $m$ ) of $X$, let $D_{P}$ be a local divisor defined by a local section of $\mathscr{O}\left(-K_{X}\right)$ at $P$ so that $D_{P} \cap C=\{P\}$. Then $-K_{X}-\sum_{P} D_{P}$ is a Cartier divisor and

$$
\sum_{P}\left(D_{P} \cdot C\right) \equiv\left(-K_{X} \cdot C\right) \text { modulo } \mathbb{Z} \text {. }
$$

If one can choose $D_{P}$ 's such that $\sum\left(D_{P} \cdot C\right) \leq 1$, then $\sum\left(D_{P} \cdot C\right)=\left(-K_{X} \cdot C\right)$ (0.4.10.4) and this shows that $\sum D_{P} \in\left|-K_{X}\right|$. 
Let us work on Example (0.4.12.4).

\section{(0.4.14.2) Local description.}

(i) Let $P$ be as in Example (0.4.12.4). Then $\mathscr{O}\left(-K_{X}\right)$ near $P$ is the invariant part of $\mathscr{O}_{X^{\sharp}}(d x \wedge d y \wedge d z)^{-1}$. Since $\left(y-x^{a}\right) /(d x \wedge d y \wedge d z)$ is $\boldsymbol{\mu}_{m}$-invariant, it is a local section of $\mathscr{O}\left(-K_{X}\right)$ and defines $D_{P}=\left\{y=x^{a}\right\} / \boldsymbol{\mu}_{m}$. Then

$$
\left(D_{P} \cdot C\right)=\frac{1}{m}\left(\left\{y=x^{a}\right\} \cdot(x \text {-axis })\right)=\frac{a}{m}<1 .
$$

(ii) If $P$ in (i) is the only point of $X \supset C$ with index $>1$, then $D_{P} \in\left|-K_{X}\right|$ and $D_{P} \simeq\left(\mathbb{C}^{2}, 0\right) / \mu_{m}$ with $\zeta(x, z)=\left(\zeta x, \zeta^{-1} z\right)$. So $D_{P}$ has a rational double point of type $A_{m-1}$ at $P$.

(0.4.14.3) One can argue similarly with $\mathscr{O}\left(-2 K_{X}\right)$. These methods work when $X$ has at most one point $P$ with index $>2$ on $C$. We should point out that the computation is needed only at $P$ (note: $-2 K_{X}$ is Cartier on $X-P$ ) and it is similar to $(0.4 .14 .2)$.

(0.4.15) A global method to find members $\in\left|-K_{X}\right|$. This part is done in $\S 9$. In view of the classification (0.4.13.1) and the local methods (0.4.14.3), we only treat $X \supset C \simeq \mathbb{P}^{1}$ with exactly two singular points, which are of indices $\geq 3$. In this case, an isomorphism

$$
\operatorname{gr}_{C}^{0} I \simeq \mathscr{O} \oplus \mathscr{O}(-1)
$$

is proved and the singularities of $X \supset C$ and the infinitesimal structure of $X$ along $C$ are further studied by the arguments (9.1)-(9.8) which are similar to but more elaborate than (0.4.13.2). Let us concentrate on the ordinary case for simplicity. (The nonordinary case is treated similarly.) Here are the results.

(0.4.15.1) Further classification. Let $X \supset C \simeq \mathbb{P}^{1}$ be an extremal nbd with two ordinary points of indices $\geq 3$. Then

(i) $X \supset C$ at each ordinary point $P$ is given by Example (0.4.12.4) for suitable $a$, and furthermore for suitable choices of coordinates in (0.4.12.4) at these points one has

(ii) the (unique) direct summand $\mathscr{L}=\mathscr{O} \subset \operatorname{gr}_{C}^{0} I \simeq \mathscr{O} \oplus \mathscr{O}(-1)$ restricts to $\mathscr{O}_{C}(x z) \subset \mathscr{O}_{C}\left(x^{m-a} y\right) \oplus \mathscr{O}_{C}(x z)$ at each $P$, and

(iii) there is a direct summand $\mathscr{M} \simeq \mathscr{O}(-1)$ of $\operatorname{gr}_{C}^{0} I$ restricting to $\mathscr{O}_{C}\left(x^{m-a} y\right)$ at each $P$.

This might not sound strong enough to be called a classification. However, there is an explicit description of all the extremal nbds $X \supset C \simeq \mathbb{P}^{1}$ with two points of indices $\geq 3$ based on (0.4.15.1), which will be published elsewhere.

We note that an explicit local description of this kind is the core of the $\ell$-structure introduced in $\S 8$. Out of this, one can pick up a homomorphism $\omega_{X} \rightarrow \operatorname{gr}_{C}^{0} I$ inducing an isomorphism $\operatorname{gr}_{C}^{0} \omega \simeq \mathscr{M}$. Then we extend it to $\omega_{X} \rightarrow I_{C} / I_{C}{ }^{(n)}$ for all $n>0$ in (9.9). 
Let us illustrate the extension mechanism by arguing backward. From now on till the end of (0.4.15.5), the sheaves $\mathscr{L}$ and $\mathscr{M}$ are chosen and fixed as in (0.4.15.1).

(0.4.15.2) What is to be expected. Assume that a member $D(\supset C)$ of $\left|-K_{X}\right|$ is the zero locus of a homomorphism $\omega_{X} \rightarrow I_{C}$ extending $\omega_{X} \rightarrow \mathscr{M} \subset \operatorname{gr}_{C}^{0} I$ above. Then, for suitable (formal) coordinates as in $(0.4 .15 .1), D$ restricts to $D_{P}=\{y=0\} / \mu_{m}$ near each $P$ (an exercise using (0.4.15.1(iii))). This $D_{P}$ has only rational double points as in (ii) of (0.4.14.2) and $D$ is smooth outside the ordinary points.

The Weil divisors $n C$ of $D(n>0)$ are defined in $X$ (not $D$ ) by ideals $J_{n}\left(\subset \mathscr{O}_{X}\right)$. For an ideal $J \subset \mathscr{O}_{X}$, we denote by $F^{1} J$ the kernel of the natural map $J \rightarrow \operatorname{gr}_{C}^{0}(J)$. We note that $\operatorname{gr}_{C}^{0}(J)=J / F^{1} J$ and $F^{1} J \supset I_{C} J$.

By the construction, $J_{2}$ is the ideal uniquely determined by $I_{C} \supset J_{2} \supset I_{C}{ }^{(2)}$ and $J_{2} / I_{C}{ }^{(2)}=\mathscr{M} \subset \operatorname{gr}_{C}^{0} I$, and the ideals

$$
J_{2} \supset J_{3} \supset \cdots \supset J_{n} \supset \cdots
$$

enjoy the following properties $(n \geq 2)$.

(a) ${ }_{n} \operatorname{gr}_{C}^{0}\left(J_{n}\right)=J_{n} / F^{1} J_{n}$ is a locally free $\mathscr{O}_{C}$-module of rank 2 ,

(b) ${ }_{n}$ the inclusion $J_{n} \subset J_{2}$ induces a natural surjection

$$
\lambda_{n}: \operatorname{gr}_{C}^{0}\left(J_{n}\right) \rightarrow \mathscr{M} \quad\left(\subset \operatorname{gr}_{C}^{0} I\right)
$$

( $\lambda_{n}$ corresponds to $\alpha_{n}$ in (9.9.6)) fitting in the exact sequence

$$
E_{n}: 0 \rightarrow \operatorname{Ker} \lambda_{n} \rightarrow \operatorname{gr}_{C}^{0}\left(J_{n}\right) \stackrel{\lambda_{n}}{\longrightarrow} \mathscr{M} \rightarrow 0,
$$

(c) ${ }_{n+1} J_{n} \supset J_{n+1} \supset F^{1} J_{n}$, and $J_{n+1} / F^{1} J_{n}$ is an invertible subsheaf $\mathscr{M}_{n}$ of $\mathrm{gr}_{C}^{0}\left(J_{n}\right)$ giving a splitting of $E_{n}$.

Remark. We note that $\operatorname{deg} \operatorname{Ker} \lambda_{n} \geq 0$ by $(\mathrm{a})_{n},(\mathrm{~b})_{n},(\mathrm{c})_{\nu}(\nu \leq n)$. Indeed $J_{n} \supset I_{C}{ }^{n}$ by $(\mathrm{c})_{\nu}$ 's, and the multiplication $\otimes^{n}\left(I_{C}\right) \rightarrow J_{n}$ induces a homomorphism $S^{n}\left(\operatorname{gr}_{C}^{0} I\right) \rightarrow \operatorname{gr}_{C}^{0}\left(J_{n}\right)$ and an injection $\mathscr{O} \simeq \mathscr{L}^{n} \hookrightarrow \operatorname{Ker} \lambda_{n}$ (0.4.15.1). In particular, $\operatorname{deg} \operatorname{Ker} \lambda_{n}>\operatorname{deg} \mathscr{M}=-1$ hence $E_{n}$ is split.

Here is an explicit description at each ordinary point $P$.

(0.4.15.3) Local description. There is a (formal) coordinate system $(x, y, z)$ as in (0.4.15.1) with the following for all $n$ :

(d) ${ }_{n} J_{n}$ is the invariant part of $\left(y, z^{n}\right)$ at $P$, and

(e) ${ }_{n} \operatorname{gr}_{C}^{0}\left(J_{n}\right)=\mathscr{O}_{C}\left(x^{m-a} y\right) \oplus \mathscr{O}_{C}\left(x^{n-m[n / m]} z^{n}\right)$ at $P$, and $\lambda_{n}$ is the projection to the first factor $\mathscr{M}=\mathscr{O}_{C}\left(x^{m-a} y\right)$.

We note that $(e)_{n}$ follows from (d) $)_{n}$ easily.

(0.4.15.4) The extension step. Now without $D$, we inductively construct $J_{n}$ 's from $J_{2}$ with the properties $(\mathrm{a})_{n}, \ldots,(\mathrm{d})_{n}$. If $J_{\nu}$ with the properties $(\mathrm{a})_{\nu}, \ldots$, $(\mathrm{d})_{\nu}$ are chosen $(\nu \leq n)$, then $\operatorname{Ker} \lambda_{n} \geq 0$ and $E_{n}$ is split by the remark in 
(0.4.15.2). Let $\mathscr{M}_{n} \subset \operatorname{gr}_{C}^{0}\left(J_{n}\right)$ be an arbitrary splitting submodule for $E_{n}$, and $J_{n+1}$ as in $(\mathrm{c})_{n+1}$. Then $(\mathrm{a})_{n+1}$ and $(\mathrm{b})_{n+1}$ are satisfied. Hence to satisfy $(\mathrm{d})_{n+1}$, we will choose a proper $\mathscr{M}_{n}$ using $\operatorname{deg} \operatorname{Ker} \lambda_{n} \geq 0$ above.

(0.4.15.5) Local description. Let us illustrate this using the coordinates in $(0.4 .15 .1)$. Let $n \geq 2$. Assume that $(\mathrm{d})_{n}$ holds. $\left((\mathrm{d})_{2}\right.$ follows from (iii) of $(0.4 .15 .1)$.) Via $(e)_{n}$, we have

$$
\mathscr{M}_{n}=\mathscr{O}_{C}\left(x^{m-a} y+c x^{n-m[n / m]} z^{n}\right) \text { near } P
$$

for some $c \in \mathbb{C}\left\{x^{m}\right\}$. For $(\mathrm{d})_{n+1}$ to hold, $c$ has to satisfy certain conditions. Let us illustrate it when $n \equiv 0(m)$ by treating the cases $c=0,1$ to avoid inessential coordinate changes.

Case $c=0$. One has $\mathscr{M}_{n}=\mathscr{O}_{C}\left(x^{m-a} \cdot y\right)$ and $J_{n+1}$ is the invariant part of $\left(y, z^{n+1}\right)=(y)+(y, z)\left(y, z^{n}\right)$.

Case $c=1$. One has $\mathscr{M}_{n}=\mathscr{O}_{C}\left(x^{m-a} y+z^{n}\right)$ and $J_{n+1}$ is the invariant part of $\left(y^{2}, y z, z^{n+1}, x^{m-a} y+z^{n}\right)=\left(x^{m-a} y+z^{n}\right)+(y, z)\left(y, z^{n}\right)$.

Thus $(\mathrm{d})_{n+1}$ does not hold in the case $c=1$ when $n \equiv 0 \quad(m)$, and the choice of $\mathscr{M}_{n}$ should not be arbitrary. In general, one can see that $(\mathrm{d})_{n+1}$ is satisfied if $c(0)=0$. Hence we can choose an $\mathscr{M}_{n}$ satisfying the two conditions (there are two points) because $\mathscr{M}_{n}$ depends on two parameters by $\operatorname{deg} \operatorname{Ker} \lambda_{n}>$ $\operatorname{deg} \mathscr{M}=-1$.

Though we use these $J_{n}$ 's to compute the general sections of $\omega_{X}{ }^{*} \otimes I_{C} / I_{C}{ }^{(n)}$ for $n \gg 0$ in (9.9.6)-(9.9.13), it is intuitively clearer to say that the good member $D$ of $\left|-K_{X}\right|$ can be recovered (cf. (0.4.15.2)) as the formal Weil divisor $\lim C_{n}$ of the completion $X^{-}$of $X$ along $C$, where $C_{n}$ is the closed subscheme defined by $J_{n}$.

(0.4.16) Comment on imprimitive points. A point $P$ on an extremal nbd $X \supset$ $C \simeq \mathbb{P}^{1}$ is imprimitive (1.7) if

$$
\stackrel{\lim }{\longleftarrow} \pi_{1}(U \cap C-P) \simeq \mathbb{Z} \rightarrow \underset{\lim }{\longleftarrow} \pi_{1}(U-P) \simeq \mathbb{Z} /(\text { index } P)
$$

(cf. $(0.4 .12))$ is not surjective.

If $X \supset C \simeq \mathbb{P}^{1}$ has an imprimitive point $P$, then $X \supset C$ has a finite cover $X^{\prime} \supset C^{\prime}$ which is an extremal nbd primitive everywhere along any irreducible component of $C^{\prime}$ ( $C^{\prime}$ is reducible). Therefore we may work on $X^{\prime}$ instead of $X$. Nevertheless we treat imprimitive points as extra cases and classify them. It is not just because we want to classify them. Our proof of the flip theorem needs them. Indeed if we do not flip the imprimitive $X$ directly, we have to flip several curves on $X^{\prime}$ instead, since $C^{\prime}$ above is reducible. If the 3 -fold picks up an imprimitive point after one flip, then one has to go up to a finite cover of the flipped 3-fold. Therefore, we need to disprove the possibility:

(0.4.16.1) One has to go up to covers for infinitely many times and hence the processes do not stop. 
The impossibility of (0.4.16.1) is not obvious to us. We can settle it, but our proof even uses the flip theorem.

(0.4.17) The descriptions of the sections. Since $\S \S 6,7$, and 9 are described in (0.4.13), (0.4.14), and (0.4.15), let us now describe other sections. $\S 1$ gives basic definitions and the corollaries to the vanishing $(0.4 .6)$. $\$ 1$ a reviews the necessary material on terminal singularities. The terminal lemma (1a.6) in it is used all over in this paper and is the key in the number matching calculation mentioned above. $\S 1 \mathrm{~b}$ gives us some tools to deform extremal nbds (cf. (0.4.12.2)), and it says that being an extremal nbd is an open condition. $\S \S 2$ and 8 prepare basic formulas (cf. (0.4.10) and (0.4.11)) and terminology (e.g. $l$-structures mentioned in $(0.4 .15 .1)$ ), and $\$ 2$ gives formulas covering all the cases while $\S 8$ gives carefully tuned formulas covering only the delicate case. $\S \S 3$ and 4 classify the local structure of $X \supset C$ at a singular point $x$ of $X$ into several cases (cf. (0.4.12) and Appendix A), and $\S 5$ gives practical formulas to work with in $\S \S 6$ and $9 . \S 10$ states a slight generalization of our main theorem (0.4.5) together with a short explanation and it also makes comments on (1a.7).

We put a diagram indicating logical dependence among sections:

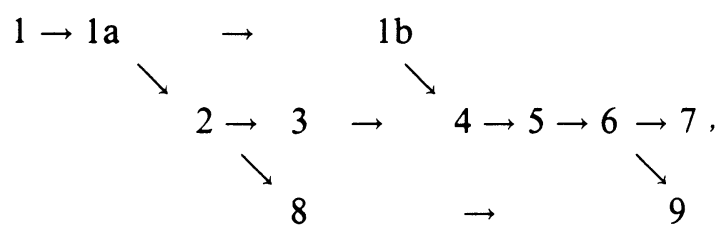

where $X \rightarrow \cdots \rightarrow Y$ means that $\S \mathrm{Y}$ depends on some results in $\S \mathrm{X}$. For instance, it does not mean that the results in $\S 1 \mathrm{~b}$ are quoted only in $\S 4$; they are also quoted in $\S 9$.

Acknowledgments . I would like to express my sincere thanks to Professor Kollár, a conversation with whom was invaluable for finishing the final step. I am grateful to Professors T. Farrell, Kuranishi, Moishezon, and Mok who helped me to work in the category of analytic spaces (rather than formal schemes), and also to Professor Shepherd-Barron who helped me in §7. I am grateful to Professor D. Morrison for his generous linguistic advice.

I would like to express my indebtedness to Professor Mumford for communicating to me his insight (which has inspired my work for eight years) that the method of [Mrl] should lead to a numerical condition for uniruledness.

During the past six years, I have been working on this problem at the following institutions: Harvard University, under the partial support of Japan U.S. Foundation; the Institute for Advanced Study at Princeton, under the partial support of the Alfred P. Sloan Foundation; the Max-Planck Institut für Mathematik, under the partial support of the SFB-40; Nagoya University; and Columbia University. I am very grateful to them for their support and hospitality; to Professors Hironaka, Hirzebruch, and Pinkham for helping with arrangements; and finally to Nagoya University for giving me these opportunities. 
I should add gratefully that the referee's constructive criticism and patience helped me revise the introduction very much.

Conventions. A variety (or an algebraic variety) means an irreducible reduced

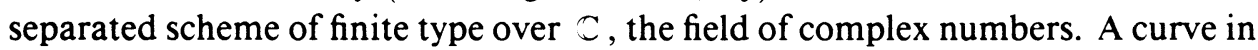
a variety means a 1-dimensional closed subscheme. An analytic space is meant to be Hausdorff and have countable open bases. The word "bimeromorphic" is used interchangeably with "birational" by abuse of language.

"Complex" and " 0 -sequence" are used interchangeably.

The set of rational numbers is denoted by $\vec{\xi}$; the set of real numbers is denoted by $\mathbb{Z}$; the set of integers is denoted by $\mathbb{Z}$; the set of nonnegative integers $\{z \in \mathbb{Z} \mid z \geq 0\}$ is denoted by $\mathbb{Z}_{+}$; and the set of positive integers $\{z \in \mathbb{Z} \mid z>0\}$ is denoted by $\mathbb{Z}_{++}$and it is equal to $\mathbb{I !}$ which denotes the set of natural numbers.

For $r \in \mathbb{Z},[r]$ denotes the integer $d$ such that $d \leq r<d+1$, and $\lceil r\rceil$ the integer $u$ such that $u-1<r \leq u$. For $i, j \in \mathbb{Z}, \delta_{i, j}$ denotes Kronecker's $\delta$, i.e.

$$
\delta_{i, j}= \begin{cases}1 & \text { if } i=j, \\ 0 & \text { if } i \neq j .\end{cases}
$$

For $m \in \mathbb{I}$, we set $\mathbb{Z}_{m}=\mathbb{Z} / m \mathbb{Z}$ and $\boldsymbol{\mu}_{m}=\left\{z \in \mathcal{I} \mid z^{m}=1\right\}$.

List of notation and terminology. This list can be found at the end of the paper.

\section{Preliminaries and basic Definitions}

The main purpose of this section is to recall some known results and make basic definitions together with auxiliary results.

(1.0) Definition (Reid [R1]). Let $(X, P)$ be a germ of a normal analytic (or algebraic) variety. Let $f: Y \rightarrow(X, P)$ be a resolution, i.e. a proper bimeromorphic morphism from a complex manifold $Y$. We say that the singularity $(X, P)$ is rational if $R^{i} f_{*} \mathscr{O}_{Y}=0$ for all $i>0$ and all resolutions $f: Y \rightarrow(X, P)$. We say that $(X, P)$ is a canonical (resp. terminal) singularity if

(i) there is an integer $r>0$ such that $r K_{X}$ is a Cartier divisor (the smallest such $r$ is called the index of $(X, P))$, and

(ii) let $f: Y \rightarrow(X, P)$ be an arbitrary resolution, and let $E_{1}, \ldots, E_{n}$ be all the exceptional divisors. Then one has $r K_{Y}=f^{*}\left(r K_{X}\right)+\sum_{i} a_{i} E_{i}$ with all $a_{i} \geq 0$ (resp. $a_{i}>0$ ).

Let us introduce the notion of an extremal nbd which we will study throughout this paper.

(1.1) Let $X$ be an analytic 3-fold with at most terminal singularities and $C \subset X$ a reduced connected curve, and we consider the germ of $X$ along $C$. We say that $X \supset C$ is an extremal $n b d$ if there is a proper bimeromorphic morphism $f: X \rightarrow(Y, Q)$ so that $f_{*} \mathscr{O}_{X}=\mathscr{O}_{Y}, f^{-1}(Q)=C$ (as sets), $\left.f\right|_{X-C}$ 
is an isomorphism, and $-K_{X}$ is $f$-ample. We call $f: X \rightarrow(Y, Q)$ the total contraction. (In case of reducible $C, f$ contracts all the components of $C$, which is why we put the adjective "total.") We note that $X$ is the blow-up of $(Y, Q)$ by a divisorial ideal $f_{*} \mathscr{O}\left(-m K_{X}\right) \simeq \mathscr{H} m\left(\omega_{Y}^{\otimes_{m}}, \mathscr{O}_{Y}\right)$ for sufficiently divisible $m \in \mathbb{N}$, because $X \rightarrow Y$ is an isomorphism in codimension 1 . Since $(Y, Q)$ is an isolated singularity, it is algebraic by Artin [A2], hence so is $f: X \rightarrow Y$. We note that our notion of total contraction of extremal nbd coincides with Kawamata's flipping singularity [Ka2, $\S 8]$. We quote his result $[\mathrm{Ka} 2, \S 8]$ with the Grauert-Riemenshneider vanishing theorem [GR].

(1.2) Theorem. $R^{i} f_{*} \mathscr{O}_{X}=R^{i} f_{*} \omega_{X}=0 \quad(i>0)$.

(1.2.1) Remark. For an arbitrary ideal $J$ such that $\operatorname{Supp} \mathscr{O}_{X} / J \subset C$, one sees

$$
H^{1}\left(\mathscr{O}_{X} / J\right)=H^{1}\left(\omega_{X} / J \omega_{X}\right)=H^{1}\left(\left(\omega_{X} / J \omega_{X}\right) / \mathscr{T}_{\circ r}\right)=0
$$

by (1.2) because fibers of $f$ have dimension $\leq 1$, where $\mathscr{T}_{o r}$ denotes the largest submodule of $\omega_{X} / J \omega_{X}$ of finite length.

The following two corollaries are in [BS, $\S 8, \mathrm{Ka} 2, \S 3]$. We give the proofs for the readers' convenience.

(1.3) Corollary. (i) $C$ is a union of $\mathbb{P}^{1} s$, whose configuration is a tree.

(ii) If $C$ is a union of $r \mathbb{P}^{1,} s(r \geq 1)$, then

$$
\operatorname{Pic} X \simeq H^{2}(C, \mathbb{Z}) \simeq \mathbb{Z}^{\oplus r} .
$$

Since (1.3(ii)) is used in this paper only to compare a finite number of divisors at a time, we simply shrink $X \supset C$ if needed and we need not worry about the inductive limit.

Proof. (i) follows from the exact sequence $R^{1} f_{*} \mathscr{O}_{X} \rightarrow H^{1}\left(C, \mathscr{O}_{C}\right) \rightarrow 0$. From (1.2) and the exact sequence

$$
0 \rightarrow \mathbb{Z}_{X} \rightarrow \mathscr{O}_{X} \rightarrow \mathscr{O}_{X}^{*} \rightarrow 1
$$

we have Pic $X \simeq R^{2} f_{*} \mathbb{Z}_{X} \simeq H^{2}\left(C, \mathbb{Z}_{C}\right) \simeq \mathbb{Z}^{\oplus r}$, since $X$ is a germ along $C$ [Go, Théorème 4.11.1].

(1.4) Corollary. If $L$ is an invertible sheaf on $X$ such that $\left(L \cdot C_{i}\right) \geq 0$ for all irreducible components $C_{i}$, then $f^{*} f_{*} L \rightarrow L$ and $R^{1} f_{*} L=0$.

Proof. For each $i$, choose $P_{i} \in C_{i}$ so that $P_{i} \notin C_{j}$ for all $j \neq i$, and a Cartier divisor $H_{i}$ of $X$ such that $H_{i} \cdot C_{i}=P_{i}$ and $H_{i} \cap C_{j}=\varnothing$ for all $j \neq i$ (note that $X$ is a germ along curve $C$ ). Then $L \simeq \mathscr{O}\left(\sum_{i}\left(L \cdot C_{i}\right) H_{i}\right)$ by (1.3). By choosing different $P_{i}$ 's, one obtains a surjection $\mathscr{O}_{X}{ }^{\oplus 2} \rightarrow L$ and hence $f^{*} f_{*} L \rightarrow L$. Since $\operatorname{dim} f^{-1}(Q)=1$, one has $0=R^{1} f_{*} \mathscr{O}_{X}{ }^{\oplus 2} \rightarrow R^{1} f_{*} L$.

(1.5) Corollary. If $C^{\prime}$ is a curve contained in $C$, then the germ of $X$ along $C^{\prime}$ is an extremal $n b d$. 
Proof. Under the notation of proof of (1.4), the morphism associated to the base-point-free linear system $\left|\sum_{i \in I} H_{i}\right|$ contracts components of $C^{\prime}$ only, where $I=\left\{i \in I \mid C_{i} \not \subset C^{\prime}\right\}$.

We want to study the subgroup of the divisor class group $\mathrm{Cl} X$ consisting of Weil divisor classes $D$ which are $\mathbb{Q}$-Cartier (i.e. $n D$ is Cartier for some $n \in \mathbb{N}$ ). We call this the semi-Cartier divisor class group and denote it by $\mathrm{Cl}^{\mathrm{sc}} X$. Then we quote

(1.6) Proposition (Reid, Ue [Ka2, Lemma 5.1]). If $(V, P)$ is a 3-fold terminal singularity of index $m$, then $\mathrm{Cl}^{\mathrm{sc}}(V, P) \simeq \mathbb{Z} /(m)$ and it is generated by the class $\left[\omega_{V}\right]$ of $\omega_{V}$.

We note that (1.6) implies that if $P$ is a terminal singular point of index $m$ of an algebraic (or even formal) 3 -fold $V$ then $\mathbb{Z} /(m)\left[\omega_{V}\right]$ is equal to the torsion part of $\mathrm{Cl} \mathscr{O}_{V, P}$.

(1.7) Corollary-Definition. Under the notation and assumptions of (1.6), let $(W, P)$ be a smooth curve $\subset(V, P)$. Then

(i) for an arbitrary $\xi \in \mathrm{Cl}^{\mathrm{sc}}(V, P)$, there exists an effective divisor $D(\ni P)$ such that $[D]=\xi$ and $D \cap W=\{P\}$.

(ii) $\xi \mapsto(m D \cdot C) / m$ induces a well-defined homomorphism

$$
\operatorname{cl}(W, P): \mathrm{Cl}^{\mathrm{sc}}(V, P) \rightarrow \frac{1}{m} \mathbb{Z} / \mathbb{Z} \subset \mathbb{Q} / \mathbb{Z} .
$$

We say that $V \supset W$ is locally primitive at $P$ (and $P$ is a primitive point) if $\operatorname{cl}(W, P)$ is an isomorphism and that an extremal nbd $X \supset C \simeq \mathbb{P}^{1}$ is locally primitive if it is so at each point of $C$. A point $P \in W$ is called imprimitive if $V \supset W$ is not primitive at $P$, and

(iii) let

$$
\pi:\left(V^{\sharp}, P^{\sharp}\right) \rightarrow(V, P)
$$

be the canonical cover (cf. [R2]) and $W^{\sharp}=\pi^{-1}(W)_{\text {red }}$. Then $W^{\sharp}$ has exactly $|\operatorname{Ker} \mathrm{cl}(W, P)|$ irreducible components. In particular, $V \supset W$ is locally primitive iff $W^{\sharp}$ is irreducible. We call $|\operatorname{Kercl}(W, P)|($ resp. $m /|\operatorname{Kercl}(W, P)|)$ the splitting degree (resp. subindex) of $V \supset W$ at $P$ (or simply of $P$ ).

Proof. Let $I \subset \mathscr{O}_{V, P}$ be a divisorial ideal representing $-\xi$. Let $\phi$ be an element of $\mathscr{H}=m\left(I, \mathscr{O}_{V}\right)$ which does not vanish along any irreducible components of $(W, P)$. Then the divisor $D$ defined by $\phi I$ satisfies the conditions in (i). Let $D$ be an effective Weil divisor with class $\left[\omega_{V}\right]$ such that $D \cap W=\{P\}$ as in (i). Let $\phi \in \mathscr{O}_{V, P}$ be such that $(\phi)=m D$ and $n=(m D \cdot W)$. Then $\left.\phi\right|_{W}=t^{n}$ for some coordinate $t$ of $(W, P)$, and $|\operatorname{Kercl}(W, P)|=(m, n)$. One sees that $\mathscr{O}_{W^{\sharp}, P} \supset \mathscr{O}_{W, P}[z] /\left(z^{m}-\left.\phi\right|_{W}\right)=\mathbb{C}\{t\}[z] /\left(z^{m}-t^{n}\right)$ is birational because $V^{\sharp} \rightarrow\left\{(v, z) \in V \times \mathbb{C} \mid z^{m}=\phi\right\}$ is isomorphic above general points of $W$. Thus $W^{\sharp}$ has $(m, n)$ irreducible components. 
(1.8) Let $X \supset C \simeq \mathbb{P}^{1}$ be an extremal nbd. Let $H$ be an effective Cartier divisor on $X$ such that $(H \cdot C)=1$ and $H \cap C$ is a smooth point of $X$. Let $P_{1}, \ldots, P_{n} \in C$ be all the points of $X$ of indices $>1$ (say, $m_{1}, \ldots, m_{n}$ ) and $D_{1}, \ldots, D_{n}$ effective $\mathbb{Q}$-Cartier divisors such that $D_{i} \cap C=\left\{P_{i}\right\}$ and $\left[D_{i}\right]$ is a generator of $\mathrm{Cl}^{\mathrm{sc}}\left(X, P_{i}\right)$ for all $i$ (1.7). Then these imply that the following natural 0 -sequence is an exact sequence

$$
0 \rightarrow \operatorname{Pic} X \rightarrow \mathrm{Cl}^{\mathrm{sc}} X \rightarrow \bigoplus_{i} \mathrm{Cl}^{\mathrm{sc}}\left(X, P_{i}\right) \rightarrow 0,
$$

and one has

(1.9) Corollary. $\mathrm{Cl}^{\mathrm{sc}}(X)$ is generated by $\left[D_{1}\right], \ldots,\left[D_{n}\right]$ and $[H]$, and $m_{i}\left[D_{i}\right]-\left(m_{i} D_{i} \cdot C\right)[H]=0 \quad(1 \leq i \leq n)$ are the generating relations, and

(1.10) Corollary. Under the notation and assumptions of (1.8), the following are equivalent.

(i) $(D \cdot C)=1 / m_{1} \cdots m_{n}$ for some $D \in \mathrm{Cl}^{\mathrm{sc}}(X)$.

(ii) $\mathrm{Cl}^{\mathrm{sc}}(X) \simeq \mathbb{Z}$.

(iii) $\mathrm{Cl}^{\mathrm{sc}}(X)$ is torsion-free.

(iv) $X \supset C$ is locally primitive (i.e. $\left(m_{i},\left(m_{i} D_{i} \cdot C\right)\right)=1$ for all $\left.i\right)$ and $\left(m_{i}, m_{j}\right)=1$ for all $i$ and $j$ such that $i \neq j$.

Proof of (1.10). By $\left[\mathrm{Cl}^{\mathrm{sc}}(X)\right.$ : $\left.\mathrm{Pic} X\right]=m_{1} \cdots m_{n}$, (i) implies (ii), and (ii) $\Rightarrow$ (iii) is obvious. It remains to prove (iii) $\Rightarrow$ (iv) and (iv) $\Rightarrow$ (i). By (1.9), it is clear that $\left(m_{i},\left(m_{i} D_{i} \cdot C\right)\right)=1$ for all $i$ if $\mathrm{Cl}^{\mathrm{sc}}(X)$ is torsion-free. Thus we may assume $\left(m_{i},\left(m_{i} D_{i} \cdot C\right)\right)=1$ for all $i$, to prove (iii) $\Rightarrow$ (iv) and (iv) $\Rightarrow(\mathrm{i})$. Let $k_{i}, n_{i} \in \mathbb{Z}$ be such that $m_{i} k_{i}+\left(m_{i} D_{i} \cdot C\right) n_{i}=1$. Then $\delta_{i}=n_{i}\left[D_{i}\right]+k_{i}[H]$ $(i \in[1, n])$ and $[H]$ form a set of generators of $\mathrm{Cl}^{\mathrm{sc}}(X)$ such that $m_{i} \delta_{i}=[H]$, whence we see that the generating relations are $m_{a} \delta_{a}=[H](1 \leq a \leq n)$. Then (iii) $\Rightarrow$ (iv) is obvious by $m_{i} \delta_{i}=m_{j} \delta_{j}$. If $m_{1}, \ldots, m_{n}$ are pairwise prime, then $n_{a}=\prod_{i \neq a} m_{i}(a \in[1, n])$ generate the unit ideal $\mathbb{Z}$ and hence $\left(\prod m_{i}\right) \delta_{a}=n_{a} H_{a} \quad(a \in[1, n])$ generate Pic $X$, whence (iv) $\Rightarrow$ (i).

(1.11) Proposition-Definition. Let $X$ be a normal analytic space, $D$ a Weil divisor and $d \in \mathbb{N}$ such that $d D$ is a principal divisor $(\phi)$ for some meromorphic function $\phi$ on $X$. Let $\pi: X^{\prime} \rightarrow X$ be the normalization of $\mathscr{O}_{X}[z] /\left(z^{d}-\phi\right)$ on $X$, which is a finite Galois $\boldsymbol{\mu}_{d}$-morphism. We call it the $\boldsymbol{\mu}_{d}$-cover associated to $[D, \phi]$ and denote it by $\pi[D, d, \phi]: X[D, d, \phi] \rightarrow X$ (we may use the divisor class of $D$ instead of $D$ and may omit $\phi$ in the expression if there is no danger of confusion $)$. One has

(i) $\pi\left[\left.D\right|_{U}, d,\left.\varphi\right|_{U}\right]=\pi[D, d, \varphi] \times_{X} U$ for open sets $U$ of $X$,

(ii) the pull back of $D$ by the finite morphism $\pi[D, d]$ is the principal divisor $(z)$,

(iii) if $X$ is connected and if the exponential map $t \mapsto e^{t}$ induces a surjection exp: $H^{0}\left(\mathscr{O}_{X}\right) \rightarrow H^{0}\left(\mathscr{O}_{X}^{*}\right)$, then the number of connected components of $X[D, d]$ is exactly $d /($ order of $[D]$ in $\mathrm{Cl} X)$, and 
(iv) the morphism $\pi[D, d]$ is étale in codim 1 . Furthermore, the ramification index of $\pi[D, d]$ at points above $P \in X$ is the order of the class $(D, P) \in \mathrm{Cl}(X, P)$ induced by $D$.

Proof. (i) and (ii) are obvious. For (iii), let $n$ be the order of [D] in $\mathrm{Cl} X$ and $c$ be the number of connected components of $X[D, d]$. By the hypothesis, the $d / n$th root of $\phi$ is a meromorphic function on $X$ because $n D$ is a principal divisor. Hence $z^{d}-\phi$ has at least $d / n$ prime factors, and it is clear that $c \geq$ $d / n$. Let $Y$ be a connected component of $X[D, d]$. Then $Y \rightarrow X$ is a Galois $\boldsymbol{\mu}_{d / c}$-morphism and the norm $N_{Y / X}(z)$ of $z$ for $Y / X$ satisfies $\left(N_{Y / X}(z)\right)=$ $(d / c) D$, whence $d / c \geq n$. Thus $c=d / n$ and (iii) is done. (iv) follows from (iii) applied to a small contractible nbd of $P$.

(1.12) Corollary. Let $X \supset C$ be an extremal $n b d, f: X \rightarrow(Y, Q)$ the total contraction, and $D \in \mathrm{Cl} X$ be of order $d$. Then $X^{\sharp}=X[D, d] \supset C^{\sharp}=$ $\pi[D, d]^{-1}(C)_{\text {red }}$ is an extremal nbd and $f^{\sharp}: X[D, d] \rightarrow\left(Y^{\sharp}, Q^{\sharp}\right)=Y\left[f_{*} D, d\right]$ is the total contraction. Using the notation of $(1.8)$, we consider $\pi^{\sharp}=\pi[D, d]$ : $X^{\sharp} \rightarrow X$ in two special cases when $C \simeq \mathbb{P}^{1}(c f$. (1.10)).

(1.12.1) Case $d=\left(m_{a},\left(m_{a} D_{a} \cdot C\right)\right)>1$ for some $a \in[1, n]$ and $D=$ $\left(m_{a} D_{a}-\left(m_{a} D_{a} \cdot C\right) H\right) / d$. In this case, $P_{a}$ is an imprimitive point of splitting degree $d$, subindex $\bar{m}=m_{a} / d, \pi^{\sharp}$ is étale over $X-\left\{P_{a}\right\}, \pi^{\sharp-1}\left(P_{a}\right)$ is one point $P_{a}^{\sharp}$ of index $\bar{m}, C^{\sharp}$ is a union of $d \mathbb{P}^{1}$ 's meeting only at $P_{a}^{\sharp}$, and each irreducible component is locally primitive at $P_{a}^{\sharp}$. In this case, we call the extremal $n b d X^{\sharp} \supset C^{\sharp}$ the splitting cover of $X \supset C$ associated to $P_{a}$, and

(1.12.2) Case $X \supset C$ is locally primitive, $d=\left(m_{a}, m_{b}\right)>1$ for some distinct $a, b \in[1, n]$, and $D=\alpha D_{a}+\beta D_{b}+\gamma H$, where $\alpha, \beta, \gamma \in \mathbb{Z}$ satisfy $\left(\alpha, m_{a}\right)=$ $m_{a} / d$ and $\left(\beta, m_{b}\right)=m_{b} / d$ (cf. proof of $(1.10)$, (iii) $\Rightarrow$ (iv)). In this case, $\pi^{\sharp}$ is étale over $X-\left\{P_{a}, P_{b}\right\}, \pi^{\sharp-1}\left(P_{a}\right)$ (resp. $\pi^{\sharp-1}\left(P_{b}\right)$ ) is one point $P_{a}^{\sharp}$ (resp. $\left.P_{b}^{\sharp}\right)$ of index $m_{a} / d \quad$ (resp. $\left.m_{b} / d\right)$, and $C^{\sharp} \simeq \mathbb{P}^{1}$.

(1.12.3) Remark. In (1.12), it is easy to see that one can choose $D$ and $d$ as in (1.12.1) or (1.12.2) if $\mathrm{Cl}^{\mathrm{sc}}(X)$ has a nonzero torsion (cf. (1.10)).

Proof. We note that exp: $H^{0}\left(\mathscr{O}_{X}\right)=H^{0}\left(\mathscr{O}_{Y}\right) \rightarrow H^{0}\left(\mathscr{O}_{X}{ }^{*}\right)=H^{0}\left(\mathscr{O}_{Y}{ }^{*}\right)$ is surjective since $(Y, Q)$ is a germ. Since $D$ and $f_{*} D$ are of order $d, X^{\sharp}$ and $Y^{\sharp}$ are connected by (1.11(iii)), whence there is only one point $Q^{\sharp} \in Y^{\sharp}$ over $Q$. By construction, $f^{\sharp}$ is bimeromorphic and étale over $Y^{\sharp}-\left\{Q^{\sharp}\right\}$, whence $f^{\sharp}$ is isomorphic outside $C^{\sharp}=f^{\sharp-1}\left(Q^{\sharp}\right)$. Since $\left(Y^{\sharp}, Q^{\sharp}\right)$ is normal, $C^{\sharp}$ is connected (ZMT). Since $\pi^{\sharp}$ is étale in codimension 1 , one sees $\pi^{\sharp *} K_{X}=K_{X^{\sharp}}$, whence $X^{\sharp} \supset C^{\sharp}$ is an extremal nbd and $f^{\sharp}$ is the total contraction.

In (1.12.1), $D$ is Cartier outside $\left\{P_{a}\right\}$, and of order $d$ at $P_{a}$. Thus (1.11(iv)) implies the first part and $\pi_{1}\left(C-P_{a}\right)=0$ implies the second part. The 
last assertion follows because $\left(X^{\sharp}, P_{a}^{\sharp}\right)$ and $\left(X, P_{a}\right)$ have isomorphic canonical covers.

In (1.12.2), the proof is the same except for the assertion $C^{\sharp} \simeq \mathbb{P}^{1}$. If $C^{\sharp}$ is reducible, then each irreducible component meets some component at $P_{a}$ and some others at $P_{b}$, because $\pi^{\sharp}$ is Galois. Then $C^{\sharp}$ contains a cycle of $\mathbb{P}^{1}$ 's, whence $H^{1}\left(\mathscr{O}_{C^{\sharp}}\right) \neq 0$, which contradicts $(1.3(\mathrm{i}))$. Thus $C^{\sharp} \simeq \mathbb{P}^{1}(1.3(\mathrm{i}))$.

(1.13) Corollary. Let $X \supset C \simeq \mathbb{P}^{1}$ be an extremal nbd. Then $X \supset C$ does not have more than one imprimitive point. If it has an imprimitive point $P$, then the splitting cover $X^{\sharp} \supset C^{\sharp}$ of $X \supset C$ associated to $P$ is locally primitive along an arbitrary irreducible component of $C^{\sharp}$.

Proof. Assume that $X \supset C$ has two imprimitive points $P, P^{\prime}\left(P \neq P^{\prime}\right)$ with splitting degrees $d, d^{\prime}(>1)$, respectively. Let $D$ (resp. $D^{\prime}$ ) be the divisor as given in $(1.12 .1)$ for $P$ (resp. $\left.P^{\prime}\right)$. Let $X^{\sharp} \supset C^{\sharp}$ (resp. $X^{b} \supset C^{b}$ ) be the extremal nbd obtained from $D$ (resp. $D^{\prime}$ ) as in (1.12). Let $\pi^{\sharp}=\pi[D, d]: X^{\sharp} \rightarrow$ $X$. Since $D^{\prime}$ has order $d^{\prime}$ globally and also at $P^{\prime}, \pi^{\sharp *} D^{\prime}$ has order $d^{\prime}$ on $X^{\sharp}$ because $\pi^{\sharp}$ is étale over $X-\{P\}$ (1.12.1). Hence $X^{\dagger}=X^{\sharp}\left[\pi^{\sharp *} D^{\prime}, d^{\prime}\right]=X^{\sharp} \times_{X}$ $X^{b} \supset C^{\dagger}=C^{\sharp} \times{ }_{X} C^{b}$ is an extremal nbd. By (1.12.1), $C^{\dagger}$ is a Galois $\mu_{d} \times \mu_{d^{\prime}}-$ cover of $\mathbb{P}^{1}$ with $d d^{\prime}$ irreducible components such that each component meets $d-1$ (resp. $d^{\prime}-1$ ) other components at every point above $P$ (resp. $P^{\prime}$ ). Thus $C^{\dagger}$ contains a cycle of $\mathbb{P}^{1}$ 's and $H^{1}\left(\mathscr{O}_{C^{\dagger}}\right) \neq 0$, which contradicts $(1.3(\mathrm{i}))$. Hence $X \supset C$ has at most 1 imprimitive point, and the last assertion follows from (1.12.1).

Let $X \supset C$ be an extremal nbd and $D(\subset C)$ an arbitrary reduced curve. Let $\operatorname{gr}_{D}^{0} \omega$ be $\omega_{X} \otimes \mathscr{O}_{D}$ modulo the maximal subsheaf of finite length (which is compatible with the definition in (2.2)).

(1.14) Proposition. Let $X \supset C$ be an extremal nbd. Then

(i) $\operatorname{gr}_{D}^{0} \omega \simeq \mathscr{O}_{D}(-1)$ for each irreducible component $D\left(\simeq \mathbb{P}^{1}\right)$ of $C$, and

(ii) if $C(1), \ldots, C(r)$ are the irreducible components of $C$, then

$$
\operatorname{gr}_{C}^{0} \omega \simeq \bigoplus_{1 \leq i \leq r} \operatorname{gr}_{C(i)}^{0} \omega .
$$

Proof. (i) Let $m \in \mathbb{N}$ be such that $m K_{X}$ is a Cartier divisor. Then $\omega_{X}^{\otimes m} \rightarrow$ $\mathscr{O}\left(m K_{X}\right) \otimes \mathscr{O}_{D}$ induces $\left(\omega_{X} \otimes \mathscr{O}_{D}\right)^{\otimes m} \rightarrow\left(\operatorname{gr}_{D}^{0} \omega\right)^{\otimes m} \hookrightarrow \mathscr{O}_{D}\left(m K_{X}\right)$. Thus $\operatorname{deg} \operatorname{gr}_{D}^{0} \omega<0$ by $\left(K_{X} \cdot D\right)<0$. On the other hand, one has $H^{1}\left(\operatorname{gr}_{D}^{0} \omega\right)=0$ by (1.2.1), whence $\operatorname{gr}_{D}^{0} \omega \simeq \mathscr{O}_{D}(-1)$.

(ii) By construction, we have an injection

$$
\operatorname{gr}_{C}^{0} \omega \rightarrow \bigoplus_{1 \leq i \leq r} \operatorname{gr}_{C(i)}^{0} \omega
$$


whose cokernel $F$ is of finite length. Since $H^{1}\left(\operatorname{gr}_{C}^{0} \omega\right)=0(1.2 .1)$, we have $0 \leq \chi\left(\operatorname{gr}_{C}^{0} \omega\right)=-$ length $(F)$ by (i). Thus $F=0$.

By (1.14(ii)), we have

(1.15) Corollary. Let $X \supset C$ be an extremal nbd such that two irreducible components of $C$ meet at $P$. Then $P$ has index $>1$.

(1.16) Corollary. Let $X \supset C \simeq \mathbb{P}^{1}$ be an extremal nbd and $P$ an imprimitive point. Then $P$ has subindex $>1$.

Indeed, we immediately see (1.16) by applying (1.15) to the splitting cover of $X \supset C$ associated to $P$.

1a (APPENDiX 1a). RESUltS ON 3-FOLD TERMinAl SINGULARITIES

In Appendix 1a, we list only some of the necessary results and definitions on 3-fold terminal singularities for convenience of quotation. We refer the reader to [Mr3], or [R4] for more polished treatments.

We start by quoting a general

(1a.1) Theorem ([Fl, El2, SB]). Canonical (hence terminal) singularities are rational in all dimensions.

Let $(X, P)$ be a 3 -fold terminal singularity of index $m \geq 1$, and let $\pi^{\sharp}:\left(X^{\sharp}, P^{\sharp}\right) \rightarrow(X, P)$ be the canonical cover, which is a Galois $\boldsymbol{\mu}_{m}$-cover. Then $\left(X^{\sharp}, P^{\sharp}\right)$ is a 3-fold terminal singularity of index $1[\mathrm{R} 1,(3.1)]$, and the $\boldsymbol{\mu}_{m}$-action on $X^{\sharp}-\left\{P^{\sharp}\right\}$ is free because the singularity of $(X, P)$ is isolated. We will fix the meaning of these symbols.

The essential result is

(1a.2) Definition-Theorem (Reid [R1]). A 3-fold hypersurface singularity $(Y, Q)$ is a cDV point if a general hyperplane section $H$ through $Q$ has at most a rational double point at $Q$. Depending on the type of $H,(Y, Q)$ is said to be of type $c A, c D$, or $c E$. Then

(i) the canonical cover $\left(X^{\sharp}, P^{\sharp}\right)$ introduced above is an isolated $\mathrm{CDV}$ point. Depending on the type of $\left(Y^{\sharp}, P^{\sharp}\right),(X, P)$ is said to be of type $c A$, $c D$, or $c E$, and

(ii) a $c D V$ point $(Y, Q)$ is a canonical singularity, and if it isolated then it is a terminal singularity.

We note that $(X, P)$ is of $c A$ type iff $\left(X^{\sharp}, P^{\sharp}\right)$ is smooth $\left(c A_{0}\right.$ type) or the quadratic part of the defining equation of the normal hypersurface singularity $\left(X^{\sharp}, P^{\sharp}\right)$ has rank $\geq 2$ as a quadratic form.

From now on we assume that $m>1$. Then the $\mu_{m}$-action on $\left(X^{\sharp}, P^{\sharp}\right)$ is to be analyzed. We fix a character $\chi$ generating $X\left(\boldsymbol{\mu}_{m}\right)=\operatorname{Hom}\left(\boldsymbol{\mu}_{m}, \mathbb{C}^{*}\right)=\mathbb{Z}_{m}$. For a $\mu_{m}$-semi-invariant $v$, we write as wt $v \equiv a(\bmod m)$ if $g(v)=\chi(g)^{a} \cdot v$ for all $g \in \boldsymbol{\mu}_{m}$. For a sequence of semi-invariants $v=\left(v_{1}, \ldots, v_{r}\right)$, we write as 
wt $v \equiv\left(a_{1}, \ldots, a_{r}\right)(\bmod m)$ if $w t v_{i} \equiv a_{i}(\bmod m)$ for all $i$. (We note that $\chi$ will be chosen to be an $\ell$-character (2.5) in the text.)

The combinatorially hardest $c A_{0}$-singularity is analyzed by

(1a.3) Theorem (Danilov [Da], Morrison and Stevens [MS]). If $\left(X^{*}, P^{z}\right)$ is smooth, it is $\boldsymbol{\mu}_{m}$-isomorphic to (x-space, 0$)$ such that

$$
\text { wt } x \equiv(a, b,-a) \quad(\bmod m)
$$

for some $a, b \in \mathbb{Z}$ prime to $m$. Conversely $(x$-space, 0$) / \boldsymbol{\mu}_{m}$ for every such action is terminal.

Other cases are combinatorially easier, though one has to take the defining equation into account.

(1a.4). Theorem ([Mr3, Theorems 12, 23, 25]). If $\left(X^{*}, P^{*}\right)$ is singular, it is $\boldsymbol{\mu}_{m}$-isomorphic to a hypersurface $\phi=0$ in (x-space, 0$)$ such that

(i) $\operatorname{wt}(x, \phi) \equiv(a, b,-a, 0,0)(\bmod m)$, or

(ii) $m=4$, and $\operatorname{wt}(x, \phi) \equiv(a, b,-a, 2,2)(\bmod m)$ for some $a, b \in \mathbb{Z}$ prime to $m$.

We introduce the following notion to give a unified treatment of these three cases in (1a.3) and (1a.4).

(1a.5) Definition-Corollary. Let us consider $\boldsymbol{\mu}_{m}$-equivariant embeddings

$$
e_{x}:\left(X^{*}, P^{*}\right) \stackrel{\sim}{\longrightarrow}\{\phi=0\} \subset(x \text {-space }, 0)=\left(\mathcal{(}_{x}^{4}, 0\right)
$$

such that $x=\left(x_{1}, \ldots, x_{4}\right)$ and $\phi$ are $\boldsymbol{\mu}_{m}$-semi-invariants and wt $x_{i} \equiv 0$ $(\bmod m)$ for some $i$ if $\left(X^{*}, P^{*}\right)$ is smooth. We call such $x$ an $\ell$-coordinate system and $\phi \in \mathcal{I}\{x\}$ an $\ell$-equation of $(X, P)$. For an $\ell$-coordinate system $x$, we call the set of points in $\widetilde{\sim}_{x}^{4}$ at which the $\boldsymbol{\mu}_{m}$-action is not free the fixed axis and denote it by $F_{x}$. Then

(i) given an $\ell$-coordinate system $x, F_{x}$ is the $x_{i}$-axis for some $i$. Thus the name of fixed axis is justified, and

(ii) given two $\ell$-coordinate systems $x$ and $y$, there exists $a \mu_{m}$-isomorphism

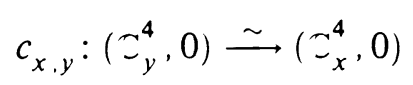

such that $c_{x, y} \circ e_{y}=e_{x}$ and hence $c_{x, y}\left(F_{y}\right)=F_{x}$. Thus the fixed axis may be denoted simply by $F$ without causing confusion, whence

(iii) given an $\ell$-coordinate system $x$ and an $\ell$-equation $\phi$, the multiplicity of 0 in the equation $\left.\phi\right|_{F}=0$ makes sense and is independent of choice of $x$ and $\phi$. We call it the axial multiplicity of $(X, P)$.

We note that this axial multiplicity coincides with Morrison's weight [Mrr2] when the singularity is not "exceptional," that is if we are not in case (1a.4(ii)). 
Proof. Indeed (i) follows from (1a.3) and (1a.4) because there exists exactly one $i$ such that wt $x_{i}$ is not prime to $m$, and (iii) immediately follows from (i) and (ii). Let $x$ and $y$ be two $\ell$-coordinate systems. Then modulo permutation of $y_{i}$ 's, we may assume that wt $x \equiv$ wt $y(\bmod m)$ and that wt $x_{4} \equiv$ wt $y_{4} \equiv 0$ $(\bmod m)$ if $\left(X^{\sharp}, P^{\sharp}\right)$ is smooth. We treat two cases for (ii).

(1a.5.1) Case $\left(X^{\sharp}, P^{*}\right)$ is smooth. We see by (1a.3) that $\mathscr{O}_{X:, P}=\mathcal{C}\left\{x^{\prime}\right\}=$ $\overparen{C}\left\{y_{1}, y_{2}, y_{3}\right\}$, and there exist semi-invariant functions $f=\left(f_{1}, \ldots, f_{4}\right) \in$ $\overparen{T}\left\{x^{\prime}\right\}$ such that $f_{i}\left(x^{\prime}\right)+\delta_{i, 4} x_{4}=y_{i}$, where $x^{\prime}=\left(x_{1}, x_{2}, x_{3}\right)$, and $\delta_{a, b}$ is Kronecker's delta. Then we set $c_{y, x}(x)=f\left(x^{\prime}\right)+\left(0,0,0, x_{4}\right)$. Since wt $f_{4} \equiv 0$ $(\bmod m)$ and $\left(\right.$ wt $\left.x_{i}, m\right)=1$ for all $i \in[1,3]$, we see $f_{4} \in\left(x^{\prime}\right)^{2}$ by $m>1$. Since $\partial\left(f_{1}, f_{2}, f_{3}\right) / \partial x^{\prime}(0) \neq 0$, we see that $c_{y, x}$ is a $\boldsymbol{\mu}_{m}$-isomorphism.

(1a.5.2) Case $\left(X^{*}, P^{\sharp}\right)$ is singular. Both $x$ and $y$ are minimal sets of generators of the maximal ideal of $\mathscr{O}_{X: P:}$. Thus there exist semi-invariant functions $f=$ $\left(f_{1}, \ldots, f_{4}\right) \in \mathbb{S}\{x\}$ such that $f_{i}(x)=y_{i}$ in $\mathscr{O}_{X:, P}$. Then $\operatorname{det} \partial f / \partial x(0) \neq 0$, and we set $c_{y, x}(x)=f(x)$. It is clear that this is a $\boldsymbol{\mu}_{m}$-isomorphism.

By (1a.3) and [Mr3, Theorems 12, 23, 25], we have

(1a.6) Theorem. Let $x$ and $\phi$ be an $\ell$-coordinate system and an $\ell$-equation of $(X, P)$. Then modulo permutation of $x_{i}$ 's we may assume that

(*) $\quad\left(\right.$ wt $\left.x_{i}, m\right)=1 \quad(i \in[1,3]), \quad$ wt $x_{1} x_{3} \equiv 0, \quad$ wt $\phi \equiv$ wt $x_{4}(m)$,

and we have two cases: (i) wt $\phi \equiv 0(m)$, or (ii) $m=4$ and wt $\phi \equiv 2$ (4). Assuming $\phi \in(x)^{2}$, we have one of the following.

(i) Case wt $\phi \equiv 0(m)$.

(i.a) $P$ is a cA-point, and up to $\boldsymbol{\mu}_{m}$-change of $\ell$-coordinate system and multiplication of $\phi$ by invariant units, we have

$$
\phi=x_{1} x_{3}+f\left(x_{2}^{m}, x_{4}\right) \text {. }
$$

(i.b) $m=3, P$ is a $c D$-point, wt $x \equiv \pm(1,1,2,0)$ ( $\bmod 3)$ modulo permutation, and $x_{4}{ }^{2}$ and $x_{3}{ }^{3}$ must appear in $\phi$ (i.e. $\partial^{2} \phi / \partial x_{4}{ }^{2}(0) \neq 0$ and $\left.\partial^{3} \phi / \partial x_{3}^{3}(0) \neq 0\right)$.

(i.c) $m=2, P$ is a $c A, c D$, or $c E_{6}$-point, wt $x \equiv(1,1,1,0)(\bmod 2)(c f$. [Mr3, Theorems 23, 25] for details ).

(ii) Case $m=4$ and wt $\phi \equiv 2$ (4). $P$ is a cA-point, wt $x \equiv \pm(1,1,3,2)$ (mod 4) modulo permutation of $x_{i}$ 's, and then $x_{3}{ }^{2}$ must appear in $\phi$ (i.e. $\left.\partial^{2} \phi / \partial x_{3}{ }^{2}(0) \neq 0\right)$.

We note that (1a.6) is only a necessary condition. Sufficient conditions are found in [Mr3, Remarks 12.1, 23.1, 25.1] and [KSB]. We quote only what we need (cf. (10.7)-(10.9)). 
(1a.7) Theorem. Let $\boldsymbol{\mu}_{m}$ act diagonally on $x$-space with $x=\left(x_{1}, \ldots, x_{4}\right)$ and $\phi \in(x)^{2}$ a $\mu_{m}$-semi-invariant so that (wt $\left.x_{i}, m\right)=1$ for $i \in[1,3]$ and wt $x_{4} \equiv$ wt $\phi(m)$. Let $(X, P)=(\{\phi=0\}, 0) / \mu_{m}$. Then

(i) ([KSB, (6.7) ], cf. also (10.7)-(10.9)) if wt $x_{4} \equiv 0 \quad(m)$ and the quadratic part $g$ of $\phi\left(x_{1}, x_{2}, x_{3}, 0\right)$ has rank $\geq 2$ as a quadratic form and if $(X, P)$ has an isolated singularity, then $(X, P)$ is terminal and of index $m$, and

(ii) ([Mr3, Remark 12.1]) if $m=4$ and $w \mathrm{t}(x, \phi) \equiv \pm(1,1,3,2,2) \bmod 4$ and if $\phi=x_{1} x_{2}+x_{3}{ }^{2}+x_{4}{ }^{2 n+1}(n \in \mathbb{N})$, then $(X, P)$ is a terminal singularity of index 4 as in (1a.6(ii)).

Idea of proof. Since (ii) is stated in [Mr3, Remark 12.11], we only comment on how to reduce (i) to a result in [KSB, (6.7)].

First we show, modulo permutation of $x_{1}, x_{2}$, and $x_{3}$ and $\boldsymbol{\mu}_{m}$-change of $x_{1} x_{2} x_{3}$-space, $g\left(x_{1}, x_{2}, 0,0\right)=x_{1} x_{2}$ in two cases.

Case 1. $m=2$ : Since wt $x_{i} \equiv 1$ (2) for $i \in[1,3]$, we may assume $g=$ $x_{1} x_{2}+\lambda x_{3}^{2}$ for some $\lambda \in \mathbb{C}$ modulo a linear change of $x_{1}, x_{2}$, and $x_{3}$, which is a $\boldsymbol{\mu}_{m}$-change of coordinates.

Case 2. $m>2$ : Since (wt $\left.x_{i}, m\right)=1$ for $i \in[1,3]$, we see no $x_{i}{ }^{2}$ appear in $g$ and that at most two of $x_{1} x_{2}, x_{2} x_{3}$, and $x_{3} x_{1}$ appear in $g$. Modulo a permutation of $x_{1}, x_{2}$, and $x_{3}$, we may write $g=\mu x_{1} x_{2}+\nu x_{1} x_{3} \quad(\mu, \nu \in \mathbb{C}$, $\mu \neq 0)$. We note that wt $x_{2} \equiv$ wt $x_{3}$ if $\nu \neq 0$. Thus $\left(x_{1}, x_{2}, x_{3}\right) \mapsto\left(x_{1}, \bar{x}_{2}, x_{3}\right)$ with $\bar{x}_{2}=\mu x_{2}+\nu x_{3}$ is a $\mu_{m}$-change of coordinates, and $g(\bar{x})=x_{1} \bar{x}_{2}$.

Then one can show that one can write

$$
\phi\left(x_{1}, x_{2}, x_{3}, x_{4}\right)=x_{1} x_{2}+f\left(x_{3}^{m}, x_{4}\right)
$$

modulo a $\boldsymbol{\mu}_{m}$-change of coordinates by approximation for some convergent power series $f[\mathrm{~A} 1]$. Now $[\mathrm{KSB},(6.7)]$ shows that $(X, P)$ is a terminal singularity of index $m$.

\section{lb (APPENDiX 1b). Deformation of eXtREMAL NBDS}

In Appendix 1b, we will study how to construct a small deformation of an extremal nbd and obtain a nearby extremal nbd from it in three steps: local process, local-to-global process, and global process. We should note that only the local description ( $1 \mathrm{~b} .3$ ) is needed for many arguments in the text but the explicit construction in (1b.8.1) is also needed for some delicate arguments (e.g. in §9). The formal scheme case is treated by [Wa].

(1b.1) Let $X \supset C \simeq \mathbb{P}^{1}$ be an extremal nbd. Let $P \in C$ be a point of $X$ of index $m$ and $\pi^{\sharp}:\left(X^{\sharp}, P^{\sharp}\right) \rightarrow(X, P)$ the canonical cover and $C^{\sharp}=$ $\pi^{\sharp-1}(C)_{\text {red }}$. Fix an embedding $(X, P) \subset\left(\mathbb{C}^{N}, P\right)$ and a $\boldsymbol{\mu}_{m}$-equivariant embedding $\left(X^{\sharp}, P^{\sharp}\right) \subset\left(\mathbb{C}^{4}, 0\right)$. 
The following are the three steps to construct a small deformation of extremal nbd $X \supset C \simeq \mathbb{P}^{1}$ with parameter $\lambda$.

(L) Construct a small embedded $\boldsymbol{\mu}_{m}$-equivariant deformation of the germs

$$
\left(X^{\sharp}, P^{\sharp}\right) \supset\left(C^{\sharp}, P^{\sharp}\right)
$$

in $\left(\mathbb{C}^{4}, P\right)$ which, if $m>1$, satisfies $(1 \mathrm{~b} .3(\mathrm{i}))$ and one of $(1 \mathrm{~b} .3(\mathrm{ii})),\left(1 \mathrm{~b} .5\left(\mathrm{ii}^{\prime}\right)\right)$ or $\left(1 \mathrm{~b} .5\left(\mathrm{ii}^{\prime \prime}\right)\right)$.

As the $\boldsymbol{\mu}_{m}$-quotients, one has a small embedded deformation $X_{P, \lambda} \supset C_{P, \lambda}$ of $(X, P) \supset C$ in $\left(\mathbb{C}^{N}, P\right)=\left(\mathbb{C}^{4}, P\right) / \mu_{m}$ with only terminal singularities, by (1b.3) and (1b.5) if $m>1$, and by Elkik's Theorem [El1] if $m=1$. Then

(LG) extend the deformation $X_{P, \lambda} \supset C_{P, \lambda}$ of the germs $(X, P) \supset(C, P)$ to a deformation $X_{\lambda} \supset C_{\lambda}$ of $X \supset C$, which is trivial outside a small nbd of $P$ (1b.8).

If $X_{\lambda} \supset C_{\lambda}$ is a small deformation of $X \supset C \simeq \mathbb{P}^{1}$, then

(G) $X_{\lambda}$ is a modification of some Stein variety if $0<|\lambda| \ll 1$ by Grauert's theorem [Gra]. Thus we have a proper bimeromorphic morphism $f_{\lambda}: X_{\lambda} \rightarrow Y_{\lambda}$ so that $C_{\lambda}$ is a part of the exceptional set $E_{\lambda}$, and $f_{\lambda}$ is the total contraction of the extremal nbd $X_{\lambda} \supset E_{\lambda}$ by (1b.10). Thus by (1.5), the germ $X_{\lambda}^{\circ} \supset C_{\lambda}$ of $X_{\lambda} \supset C_{\lambda}$ along $C_{\lambda}$ is an extremal nbd if $0<|\lambda| \ll 1$.

We call such an extremal nbd a nearby extremal nbd of $X \supset C$.

(1b.2) Let $(X, 0)$ be a 3-fold terminal singularity of index $m>1$, and $\left(X^{\sharp}, 0\right)$ the $\boldsymbol{\mu}_{m}$-canonical cover. Let $(x)=\left(x_{1}, \ldots, x_{4}\right)$ and $\phi$ be an $\ell$-coordinate system and an $\ell$-equation of $(X, P)$, and let $F^{\sharp} \subset x$-space be the fixed axis (1a.5).

(1b.3) Proposition. Let $\psi$ be a semi-invariant $\in(x) \mathbb{C}\{x\}$ such that wt $\psi \equiv$ wt $\phi(m)$ and $\{\phi=\psi=0\}$ is a surface. Let $X_{\lambda}^{\sharp}=\{\phi+\lambda \psi=0\},\left(X_{\lambda}, 0\right)=$ $\left(X_{\lambda}^{\sharp}, 0\right) / \mu_{m} \quad(\lambda \in \mathbb{C}), S^{\sharp}=\operatorname{Sing}\{\phi=\psi=0\}$, and let $S, F_{\lambda} \subset X_{\lambda}$ the images of $S^{\sharp}, F^{\sharp} \cap X_{\lambda}^{\sharp}$, respectively. Assume that

(i) $S^{\sharp} \cap F^{\sharp} \subset\{0\}$, and

(ii) $\left(X_{\lambda}, 0\right)$ is terminal if $0<|\lambda| \ll 1$.

For $\varepsilon>0$ and $\lambda \in \mathbb{C}$ such that $1 \gg \varepsilon \gg|\lambda|$, let

$$
X_{\lambda, \varepsilon}^{\sharp}=\left\{(x) \in X_{\lambda}^{\sharp}|| x_{i} \mid<\varepsilon \forall i\right\} \quad \text { and } X_{\lambda, \varepsilon}=X_{\lambda, \varepsilon}^{\sharp} / \mu_{m} \text {. }
$$

Then $X_{\lambda, \varepsilon}$ has only terminal singularities; $X_{\lambda, \varepsilon}-\left(S \cup F_{\lambda}\right)$ is smooth; $X_{\lambda, \varepsilon} \cap$ $S-F_{\lambda}$ are Gorenstein points ; $X_{\lambda, \varepsilon} \cap F_{\lambda}-(S \cup\{0\})$ consists of cyclic quotient singular points of the same index $m^{\prime}$ which is a factor of $m ; 0$ is a point of index $m$.

We note that $m^{\prime} \neq m$ in (1b.3) only if $(X, P)$ is exceptional, i.e. in case (1a.6(ii)), where $m=4$ and $m^{\prime}=2$.

Remark (1b.3.1). Under the notation and assumptions of $(1 \mathrm{~b} .3)$, let $\left(C^{\sharp}, 0\right) \subset$ $\left(X^{\sharp}, 0\right)$ be a $\boldsymbol{\mu}_{m}$-stable reduced curve such that $(C, 0)=\left(C^{\sharp}, 0\right) / \boldsymbol{\mu}_{m}$ is smooth 
and $\left(C^{\sharp}, 0\right) \mu_{m}$-equivariantly deforms with $\left(X^{\sharp}, 0\right)$ in $\mathbb{C}^{4}$ so that $C_{\lambda}^{\sharp} \subset X_{\lambda}^{\sharp}$. Then for $\varepsilon \ll 1$ and $P_{\lambda} \in C_{\lambda, \varepsilon}=\left(C_{\lambda}^{\sharp} \cap X_{\lambda, \varepsilon}^{\sharp}\right) / \mu_{m}, X_{\lambda, \varepsilon} \supset C_{\lambda, \varepsilon}$ is locally primitive at $P_{\lambda}$ iff $\left(C_{\lambda}^{\sharp}, P_{\lambda}^{\sharp}\right)$ is irreducible, where $P_{\lambda}^{\sharp} \in C_{\lambda}^{\sharp}$ is a point above $P_{\lambda}$.

Indeed this follows from $(1.7$ (iii) $)$, because $\left(X_{\lambda, \varepsilon}^{\sharp}, P_{\lambda}^{\sharp}\right) \rightarrow\left(X_{\lambda, \varepsilon}, P_{\lambda}\right)$ is the canonical cover and $\left(C_{\lambda}^{\sharp}, P_{\lambda}^{\sharp}\right)$ is the preimage of $C_{\lambda}$.

We recall a special case of Bertini's Theorem.

(1b.4) Lemma (Bertini). Let $\phi$ and $\psi$ be convergent power series in $x_{1}, \ldots$, $x_{n}$, which are prime to each other. Then $\{\phi+\lambda \psi=0\} \cap B_{\varepsilon}$ is smooth outside the singular locus of $\{\phi=\psi=0\} \cap B_{\varepsilon}$ if $0<|\lambda| \ll \varepsilon \ll 1$, where $B_{\varepsilon}=\{(x) \mid$ $\left.\left|x_{i}\right|<\varepsilon(\forall i)\right\}$.

Proof. Let $(x, \lambda)=(x(t), \lambda(t)) \quad(|t| \ll 1)$ be a parametrized curve with parameter $t$ such that $(x(0), \lambda(0))=0$ and $x(t)$ is a singular point of $\phi+$ $\lambda(t) \psi=0$ and $\lambda(t) \not \equiv 0$. It is enough to show that $x(t)$ is a singular point of $\{\phi=\psi=0\}$ for all $t$. By the hypothesis, one has

$$
\frac{\partial \phi}{\partial x_{i}}(x(t))+\lambda(t) \frac{\partial \psi}{\partial x_{i}}(x(t))=0 \quad(i \in[1, n]) .
$$

From $\phi(x(t))+\lambda(t) \psi(x(t))=0$, one has

$$
\sum_{i}\left(\frac{\partial \phi}{\partial x_{i}}+\lambda \frac{\partial \psi}{\partial x_{i}}\right) \frac{d x_{i}}{d t}+\frac{d \lambda}{d t} \psi=0
$$

whence $(d \lambda / d t) \psi(x(t))=0$ and $\psi(x(t))=0$ by $\lambda(t) \not \equiv$ const. Thus one has $\phi(x(t))=\psi(x(t))=0$, and $(*)$ above implies

$$
\operatorname{rank}\left(\left.\frac{\partial(\phi, \psi)}{\partial x}\right|_{x=x(t)}\right) \leq 1
$$

which means that $x(t)$ is a singular point of $\{\phi=\psi=0\}$ by Jacobian criterion.

Proof of (1b.3). We may assume wt $x_{4} \equiv \mathrm{wt} \phi$, wt $x_{1} x_{2} \equiv 0(m)$, (wt $\left.x_{4}, m\right)$ $\neq 1$, and wt $x_{1}$, wt $x_{2}$, wt $x_{3}$ are prime to $m$ (modulo a permutation of $\left.x_{1}, \ldots, x_{4}\right)$, so that $F^{\sharp}=x_{4}$-axis (1a.6). Since the $\mu_{m}$-action on $X^{\sharp}-\{0\}$ is free, $\left.\phi\right|_{F^{\sharp}} \not \equiv 0$. By (1b.4), one sees that $\left.(\phi+\lambda \psi)\right|_{F^{\sharp}}=0$ has no multiple roots $x_{4}$ such that $0<\left|x_{4}\right| \ll 1$ if $|\lambda| \ll 1$. Let $\varepsilon \in \mathbb{R}$ be small enough and $B_{\varepsilon}=\left\{\left(x_{1}, \ldots, x_{4}\right)|| x_{1}|, \ldots,| x_{4} \mid<\varepsilon\right\}$. Then

(1) $X_{\lambda, \varepsilon}^{\sharp}=X_{\lambda}^{\sharp} \cap B_{\varepsilon}$ is smooth in $B_{\varepsilon}-S^{\sharp}$,

(2) $X_{\lambda, \varepsilon}^{\sharp}$ has only isolated rational singularities, and

(3) $X_{\lambda, \varepsilon}^{\sharp}$ intersects transversally with $F^{\sharp}$ in $B_{\varepsilon}-\{0\}$,

if $|\lambda| \ll \varepsilon \ll 1$, where (1) follows from (1b.4), and (2) follows from [El1]. Let $\pi_{\lambda}^{\sharp}: X_{\lambda, \varepsilon}^{\sharp} \rightarrow X_{\lambda, \varepsilon}$ be the quotient map. Let $Q^{\sharp} \in X_{\lambda, \varepsilon}^{\sharp}-\{0\}$ for $\lambda$ such that $|\lambda| \ll \varepsilon$. If $Q^{\sharp} \notin F^{\sharp}$, then $\pi_{\lambda}^{\sharp}$ is étale at $Q^{\sharp}$ and $X_{\lambda, \varepsilon}$ is smooth at $\pi_{\lambda}^{\sharp}\left(Q^{\sharp}\right)$ 
by (1) if $Q^{\sharp} \notin F^{\sharp} \cup S^{\sharp}$, and has Gorenstein terminal singularity at $\pi_{\lambda}^{\sharp}\left(Q^{\sharp}\right)$ by (2) if $Q^{\sharp} \in S^{\sharp}-F^{\sharp}$. If $Q^{\sharp} \in F^{\sharp}-\{0\}$, then $x_{1}\left(Q^{\sharp}\right)=x_{2}\left(Q^{\sharp}\right)=x_{3}\left(Q^{\sharp}\right)=0$ and $x_{1}, x_{2}, x_{3}$ form a $G_{Q^{\sharp}}$-semi-invariant coordinate system for $\left(X_{\lambda, \varepsilon}^{\sharp}, Q^{\sharp}\right)$ by (3), where $G_{Q^{\sharp}}\left(\subset \mu_{m}\right)$ is the stabilizer at $Q^{\sharp}$ and $\left|G_{Q^{\sharp}}\right|=\left(\right.$ wt $\left.x_{4}, m\right)$. Since wt $x_{1} x_{2} \equiv 0(m)$ and wt $x_{1}$, wt $x_{2}$, wt $x_{3}$ are prime to $m, X_{\lambda, \varepsilon}$ has at most a terminal cyclic quotient singularity at $\pi_{\lambda}^{\sharp}\left(Q^{\sharp}\right)$ by (1a.3).

(1b.5) Corollary. In (1b.3) above, (ii) follows from either one of the following:

(ii') $(\phi, \psi) \not \subset\left(x_{1}, \ldots, x_{4}\right)^{2}$,

(ii') $(\phi, \psi) \subset\left(x_{1}, \ldots, x_{4}\right)^{2}$, wt $x_{4} \equiv 0(m),(\phi+\lambda \psi)\left(x_{1}, x_{2}, x_{3}, 0\right)$ has a quadratic part of rank $\geq 2$ in $x_{1}, x_{2}, x_{3}$ if $0<|\lambda| \ll 1$.

Proof. By (1a.3), (ii') implies (ii). Let us assume (ii"). By (1a.4(i)), (wt $\left.x_{i}, m\right)$ $=1$ for $i \in[1,3]$. Since $(X, 0)$ has an isolated singularity, $\left(X_{\lambda}, 0\right)$ has an isolated singularity if $|\lambda| \ll 1$. Then such $\left(X_{\lambda}, 0\right)$ is terminal by (1a.7(i)).

(1b.6) Proposition. Let $\mathscr{X} \supset \mathscr{C}$ be analytic subspaces of germ $\left(\mathbb{C}^{N} \times \mathbb{C}_{\lambda}^{1}, P \times 0\right)$ flat over $\left(\mathbb{C}_{\lambda}^{1}, 0\right)$ such that $C=\mathscr{C} \cap\left(\mathbb{C}^{N} \times 0\right) \subset \mathbb{C}^{N}$ is a smooth curve. Then there is a biholomorphic automorphism $\sigma$ of $\left(\mathbb{C}^{N} \times \mathbb{C}_{\lambda}^{1}, P \times 0\right)$ over $\left(\mathbb{C}_{\lambda}^{1}, 0\right)$ such that

$$
\left.\sigma\right|_{\mathbb{C}^{N} \times 0}=\mathrm{id} \text { and } \sigma(\mathscr{C})=C \times \mathbb{C}_{\lambda}^{1}
$$

Proof. We choose coordinates $\left(x_{1}, \ldots, x_{N}\right)$ of $\left(\mathbb{C}^{N}, P\right)$ such that $x(P)=0$ and $C=x_{1}$-axis. Then by flatness $\mathscr{C} \subset \mathbb{C}^{N} \times \mathbb{C}_{\lambda}^{1}$ is defined by $x_{i}-\lambda f_{i}(x, \lambda)=0$ $(i \in[2, N])$ for some convergent power series $f_{i}$. We set

$$
\sigma(x, \lambda)=\left(x_{1}, x_{2}-\lambda f_{2}, \ldots, x_{N}-\lambda f_{N}, \lambda\right) \text {. }
$$

Let $y=\left(y_{1}, \ldots, y_{N}\right)$, and let $(X, P) \subset(t y$-space, 0$) \simeq\left(\mathbb{C}^{N+1}, 0\right)$ be the germ of a pure $r$-dimensional analytic subspace such that $X \supset C=t$-axis and $(\operatorname{Sing} X) \cap C \subset\{P\}$. Then $I_{C, P} / I_{C, P}{ }^{2}$ is a rank $(r-1) \mathscr{O}_{C, P}$-module, where $I_{C, P} \subset \mathscr{O}_{X, P}$ is the defining ideal of $C$ in $(X, P)$. Let $\left(\mathscr{X}^{\prime}, P \times 0\right) \subset$ $($ ty $\lambda$-space, 0$) \simeq\left(\mathbb{C}^{N+2}, 0\right)$ be the germ of an analytic subspace defined by ideal $J \subset \mathbb{C}\{t, y, \lambda\}$ such that the projection $f:(\mathscr{X}, P \times 0) \rightarrow(\lambda$-line, 0$)$ is flat, $X=f^{-1}(0)$, and $\mathscr{X} \supset \mathscr{C}=t \lambda$-plane. Then

$$
\frac{(y)}{\lambda(y)+(y)^{2}+J} \stackrel{\sim}{\longrightarrow} \frac{(y)+(\lambda)}{(\lambda)+(y)^{2}+J} \simeq I_{C, P} / I_{C, P}{ }^{2} \quad \text { (isomorphism theorem). }
$$

Here and in (1b.7), ideals (e.g. (y)) mean ideals in $\mathbb{C}\{t, y, \lambda\}$ unless otherwise denoted. 
(1b.7) Proposition. Under the above notation and assumptions, let $u=$ $\left(u_{1}, \ldots, u_{r-1}\right)$ be elements of the ideal $(y)$ such that $I_{C, P} / I_{C, P}{ }^{2}+(u) \mathscr{O}_{X, P}$ is of finite length. Then for small enough $\alpha>\beta>0$, the natural map

$$
\mathscr{X}\left(\begin{array}{c}
I \times \gamma \times \delta \\
u<\delta
\end{array}\right)=\left\{\begin{array}{c}
(t, y, \lambda) \in \mathscr{X} ; \\
|t| \in I,|y|<\gamma \\
|u|<\delta,|\lambda|<\delta
\end{array}\right\} \rightarrow \mathscr{U}\left(\begin{array}{c}
I \times \delta \times \delta \\
u
\end{array}\right)=\left\{\begin{array}{c}
(t, u, \lambda) ; \\
|t| \in I \\
|u|,|\lambda|<\delta
\end{array}\right\}
$$

is biholomorphic for all $\gamma, \delta \in \mathbb{R}$ such that $0<\delta \ll \gamma \ll \beta$, where $|y|=$ $\operatorname{Max}_{i}\left|y_{i}\right|,|u|=\operatorname{Max}_{j}\left|u_{j}\right|$, and $I$ is the interval $(\beta, \alpha)$.

Proof. By the natural isomorphisms

$$
\begin{aligned}
\frac{J+\lambda(y)+(y)^{2}}{\lambda(y)+(y)^{2}} & \simeq \operatorname{Ker}\left(\frac{y}{\lambda(y)+(y)^{2}} \rightarrow \frac{(y)+(\lambda)}{(\lambda)+(y)^{2}+J}\right) \\
& \simeq \operatorname{Ker}\left(\bigoplus_{1 \leq i \leq N} \mathscr{O}_{C, P} y_{i} \rightarrow I_{C, P} / I_{C, P}{ }^{2}\right)
\end{aligned}
$$

one sees that $J+\lambda(y)+(y)^{2} / \lambda(y)+(y)^{2}$ is a free $\mathscr{O}_{C, P}$-module of rank $N$ $r+1$. Thus it is generated by images of some $f=\left(f_{r}, \ldots, f_{N}\right) \in J$. Let $h_{i j} \in \mathbb{C}\{t, y, \lambda\} \quad(i, j \in[1, N])$ be such that $u_{i}=\sum_{j} h_{i j} y_{j} \quad(i<r)$ and $f_{i}=\sum_{j} h_{i j} y_{j}(i \geq r)$. Since $(y) / \lambda(y)+(y)^{2}+(u)+(f)$ is of finite length, $\operatorname{det}\left(h_{i j}\right)(t, 0,0)$ is not identically zero. For arbitrary small enough $\alpha>\beta>0$, one sees $(\partial(u, f) / \partial y)(t, y, \lambda) \neq 0$ if $\alpha \geq|t| \geq \beta$ and $|y|,|\lambda| \ll \beta$, because

$$
\frac{\partial(u, f)}{\partial y}(t, 0,0)=\operatorname{det}\left(h_{i j}\right)(t, 0,0) \text {. }
$$

By the Implicit Function Theorem, $y_{j}$ 's are expressed as convergent power series $y_{j}=y_{j}(u, f)$ in $(u)$ and $(f)$ such that $y_{j}(0,0)=0$ depending holomorphically on $t$ and $\lambda$ such that $\alpha>|t|>\beta$ and $\delta>|\lambda|$ if $0<\delta \ll \beta$. In other words, $(t, u, f, \lambda)$ forms a coordinate system for the open set $\{(t, y, \lambda) \mid$ $\beta<|t|<\alpha,|y|<\gamma,|\lambda|<\delta\}$ of $\mathbb{C}^{N+1}$ if $0<\delta \ll \gamma \ll \beta$. Imposing extra conditions $f=0$ and $|u|<\delta$ and making $\delta$ smaller, we see (1b.7).

(1b.8) Definition. Let $X \supset C$ be the germ of a normal analytic space $X$ along a smooth irreducible compact curve $C$ such that $C \not \subset \operatorname{Sing} X$. Let $P \in C$ and $(X, P) \subset(t y$-space, 0$) \simeq\left(\mathbb{C}^{N+1}, 0\right)$ an embedding of a germ so that $(C, P)$ is the $t$-axis, where $y=\left(y_{1}, \ldots, y_{N}\right)$. Let $X_{\lambda} \supset C_{\lambda}$ be a deformation of pair $X \supset C$ of germs at $P$. By (1b.6), we may embed $X_{\lambda} \supset C_{\lambda}$ as $\left(\mathbb{C}^{N+1} \times \mathbb{C}_{\lambda}^{1}\right.$, $P \times 0) \supset \mathscr{X} \supset \mathscr{C}=t \lambda$-plane which are flat over $\left(\mathbb{C}_{\lambda}^{1}, 0\right)$.

Let $u=\left(u_{1}, \ldots, u_{r-1}\right)$ be elements of $(y) \mathbb{C}\{t, y, \lambda\}$ such that

$$
I_{C} / I_{C}{ }^{2}+(u) \mathscr{O}_{X} \text { is of finite length at } P \text {, }
$$

where $I_{C}$ is the sheaf of defining ideals of $C$ in $X$. Let $u(\cdot, c)=\left(u_{1}(\cdot, c), \ldots\right.$, $\left.u_{r-1}(\cdot, c)\right) \in \mathbb{C}\{t, y\}$ be the restrictions of $u$ to $\lambda=c$. 
Then applying (1b.7) to both $\mathscr{X}$ and the trivial deformation $\left(X \times \mathbb{C}_{\lambda}^{1}, P \times 0\right) \subset$ $(t y \lambda$-space, 0$)$, we see that

$$
\rho: \mathscr{X}\left(\begin{array}{c}
(\beta, \alpha) \times \gamma \times \delta \\
u<\delta
\end{array}\right) \underset{\rightarrow}{\longrightarrow}\left(X \times \mathbb{C}_{\lambda}^{1}\right)\left(\begin{array}{c}
(\beta, \alpha) \times \gamma \times \delta \\
u(\cdot, 0)<\delta
\end{array}\right)
$$

such that

$$
\rho^{*} u_{i}(\cdot, 0)=u_{i} \quad(i \in[1, r-1]),\left.\quad \rho\right|_{\mathscr{C}}=\mathrm{id}: \mathscr{C} \rightarrow t \lambda \text {-plane }
$$

by the identification

$$
\mathscr{U}\left(\begin{array}{c}
(\beta, \alpha) \times \gamma \times \delta \\
u
\end{array}\right)=\mathscr{U}\left(\begin{array}{c}
(\beta, \alpha) \times \gamma \times \delta \\
u(\cdot, 0)
\end{array}\right) \text { if } 1 \gg \alpha>\beta \gg \gamma \gg \delta>0 .
$$

Thus if $1 \gg \alpha^{\prime}>\alpha>\beta \gg \gamma \gg \delta>0$, one may patch

$$
\mathscr{X}\left(\begin{array}{c}
{[0, \alpha) \times \gamma \times \delta} \\
u<\delta
\end{array}\right) \text { and }\left(X \times \mathbb{C}_{\lambda}^{1}\right)\left(\begin{array}{c}
\left(\beta, \alpha^{\prime}\right) \times \gamma \times \delta \\
u(\cdot, 0)<\delta
\end{array}\right)
$$

via the above isomorphism to get a deformation of $X\left(\begin{array}{l}{\left[0, \alpha^{\prime}\right) \times \gamma} \\ u(\cdot, 0)<\delta\end{array}\right)$ which is trivial on $X\left(\begin{array}{c}\left(\beta, \alpha^{\prime}\right) \times \gamma \\ u(\cdot, 0)<\delta\end{array}\right)$, where

$$
X\left(\begin{array}{l}
I \times \gamma \\
v<\delta
\end{array}\right)=\{(t, y) \in(X, P)|| t|\in I,| y|<\gamma,| v \mid<\delta\}
$$

for an interval $I$. Thus replacing $X$ with a smaller nbd of $C$, we can extend this deformation to the deformation $X_{\lambda} \supset C_{\lambda}=C$ of $X \supset C$. For simplicity of notation, we set (omitting $\alpha, \beta, \gamma, \delta$ )

$$
\begin{aligned}
\mathscr{X}_{\text {out }}(u) & =X \times\{\lambda|| \lambda \mid<\delta\}-\left(X \times \mathbb{C}_{\lambda}^{1}\right)\left(\begin{array}{c}
{[0, \beta] \times \gamma \times \delta} \\
u(\cdot, 0)<\delta
\end{array}\right) \supset \mathscr{C}_{\text {out }}(u), \\
\mathscr{X}_{\text {in }}(u) & =\mathscr{X}\left(\begin{array}{c}
{[0, \alpha) \times \gamma \times \delta} \\
u<\delta
\end{array}\right) \supset \mathscr{C}_{\text {in }}(u), \\
\mathscr{X}_{\text {mid }}(u) & =\mathscr{X}_{\text {out }}(u) \cap \mathscr{X}_{\text {in }}(u) \supset \mathscr{C}_{\text {mid }}(u), \quad \text { and } \\
\mathscr{X}_{\text {all }}(u) & =\mathscr{X}_{\text {out }}(u) \cup \mathscr{X}_{\text {in }}(u) \supset \mathscr{C}_{\text {all }}(u),
\end{aligned}
$$

where $\mathscr{C}_{\text {out }}(u), \ldots, \mathscr{C}_{\text {all }}(u)$ are defined in the obvious way.

We note that $X_{\lambda} \supset C_{\lambda}=C$ is obtained by patching

$$
X_{\lambda, \text { in }}=X_{\lambda}\left(\begin{array}{c}
{[0, \alpha) \times \gamma} \\
u(\cdot, \lambda)<\delta
\end{array}\right) \quad \text { and } \quad X_{\lambda, \text { out }}=X-X\left(\begin{array}{c}
{[0, \beta] \times \gamma} \\
u(\cdot, 0)<\delta
\end{array}\right)
$$

via

$$
\rho_{\lambda}: X\left(\begin{array}{c}
(\beta, \alpha) \times \gamma \\
u(\cdot, \lambda)<\delta
\end{array}\right) \stackrel{\sim}{\longrightarrow} X\left(\begin{array}{c}
(\beta, \alpha) \times \gamma \\
u(\cdot, 0)<\delta
\end{array}\right)
$$

such that $\rho_{\lambda}{ }^{*} u_{i}(\cdot, 0)=u_{i}(\cdot, \lambda) \quad(i \in[1, r-1])$, on $X_{\lambda \text {,mid }}{ }^{\text {" }}=" X_{\lambda, \text { in }} \cap X_{\lambda \text {,out }}$, and $C_{\lambda, \text { in }}, \ldots, C_{\lambda \text {,mid }}$ are defined similarly.

We call $\mathscr{X}_{\text {all }}(u) \supset \mathscr{C}_{\text {all }}(u)$ (or $X_{\lambda} \supset C_{\lambda}$ ) the twisted extension of $\mathscr{X} \supset \mathscr{C}$ (or $\left.\left(X_{\lambda}, P\right) \supset\left(C_{\lambda}, P\right)\right)$ by $u$. 
(1b.8.2) Corollary. Let $X \supset C$ be the germ of a normal analytic space along a complete smooth irreducible curve such that $C \not \subset \operatorname{Sing} X$. Let $P \in C$. Then every deformation of germs $(X, P) \supset(C, P)$ can be extended to a deformation of $X \supset C$ so that the deformation is trivial outside some small nbd of $P$.

(1b.8.3) Proposition. Let the notation and assumptions be as in (1b.8.1). Let $I$ ( resp. $I_{\lambda}$ ) be the sheaf of defining ideals of $C\left(\right.$ resp. $\left.C_{\lambda}\right)$ in $X\left(\right.$ resp. $\left.X_{\lambda}\right)$, and let $I^{(2)}$ be the symbolic square of $I$. Then

(i) the closed subscheme of $X$ defined by $I^{(2)}$ deforms in a flat family as subschemes defined by $\left(I^{(2)}\right)_{\lambda}$ iff $\chi\left(I_{\lambda} /\left(I_{\lambda}\right)^{(2)}\right)=\chi\left(I / I^{(2)}\right)$ for each $\lambda>0$ such that $|\lambda| \ll 1$. If so, then $\left(I^{(2)}\right)_{\lambda}=\left(I_{\lambda}\right)^{(2)}$ (hence denoted by $I_{\lambda}^{(2)}$ ) and $I_{\lambda} / I_{\lambda}^{(2)}$ is a flat family of locally free sheaves on $C_{\lambda}$ over $\left(\mathbb{C}_{\lambda}^{1}, 0\right)$, and

(ii) assume that the closed subscheme defined by $I^{(2)}$ deforms and that $I / I^{(2)}$ contains an invertible subsheaf $L$ such that $L_{P}$ is generated by (the image of ) $u_{1}(\cdot, 0)$. Then $L$ deforms as invertible subsheaf $L_{\lambda}$ of $I_{\lambda} / I_{\lambda}^{(2)}$ and $L_{\lambda}$ is generated at $P_{\lambda} \quad(=P)$ by $u_{1}(\cdot, \lambda)$.

Proof. (i) The only-if part is the invariance of $\chi$ in a flat family. Assume that $\chi\left(I_{\lambda} /\left(I_{\lambda}\right)^{(2)}\right)=\chi\left(I / I^{(2)}\right)$ if $0<|\lambda| \ll 1$. Let $J$ be the sheaf of defining ideals of $\mathscr{C}_{\text {all }}(u)$ in $\mathscr{X}_{\text {all }}(u)$. Then $J \mathscr{O}_{X}=I, J^{(2)} \mathscr{O}_{X}$ is a subsheaf of $I^{(2)}$ such that len $I^{(2)} / J^{(2)} \mathscr{O}_{X}<\infty$, and

$$
J \mathscr{O}_{X_{\lambda}}=I_{\lambda} \text { and } J^{(2)} \mathscr{O}_{X_{\lambda}}=\left(I_{\lambda}\right)^{(2)} \text { if } 0<|\lambda| \ll 1 .
$$

Then $F=J / J^{(2)}$ is flat over $\left(\mathbb{C}_{\lambda}^{1}, 0\right)$ and $F_{\lambda}=F \otimes \mathscr{O}_{X_{\lambda}}=I_{\lambda} /\left(I_{\lambda}\right)^{(2)}$ if $0<$ $|\lambda| \ll 1$, and we have a surjection $F_{0} \rightarrow I / I^{(2)}$. By $\chi\left(I_{\lambda} /\left(I_{\lambda}\right)^{(2)}\right)=\chi\left(I / I^{(2)}\right)$, we see that $\operatorname{Ker}\left[F_{0} \rightarrow I / I^{(2)}\right]$ is a sheaf supported on a finite set such that

$$
\chi\left(\operatorname{Ker}\left[F_{0} \rightarrow I / I^{(2)}\right]\right)=\chi\left(F_{0}\right)-\chi\left(I / I^{(2)}\right)=\chi\left(F_{\lambda}\right)-\chi\left(I_{\lambda} /\left(I_{\lambda}\right)^{(2)}\right)=0,
$$

whence $F_{0} \simeq I / I^{(2)}, J^{(2)} \mathscr{O}_{X}=I^{(2)}$, and (i) is proved.

(ii) Since $L$ and $\mathscr{O} u_{1}(\cdot, 0)$ patch on some $X\left(\begin{array}{c}(\beta, \alpha) \times \gamma \\ u(\cdot, 0)<\delta\end{array}\right)$, the trivial extension of $L$ on $\mathscr{X}_{\text {out }}(u)$ and $\mathscr{O} u_{1}$ on $\mathscr{X}_{\text {in }}(u)$ patch on $\mathscr{X}_{\text {mid }}(u)$ because $\rho^{*} u_{1}(\cdot, 0)=$ $u_{1}$.

(1b.9) Proposition. Let $X$ be an analytic space and $C$ a compact subvariety which is a smooth irreducible curve. Then there is an open neighborhood $V$ of $C$ in $X$ and an effective Cartier divisor $E$ on $V$ such that every irreducible compact subvariety $C^{\prime}$ in $V$ with dimension $>0$ is a curve and $\left(C^{\prime} \cdot E\right)>0$.

Proof. Let $\varepsilon>\delta>0$. For each $P \in C$, let $\widetilde{U}_{P}$ be a neighborhood of $P$ in $X$ such that $\tilde{U}_{P}$ is identified with an analytic set of $D_{\varepsilon} \times D_{\varepsilon}{ }^{N}$ such that $\widetilde{C}_{P}=\tilde{U}_{P} \cap C$ and $P$ are equal to $D_{\varepsilon} \times 0$ and $0 \times 0$, respectively, where $D_{\varepsilon}=\{z \in \mathbb{C}|| z \mid<\varepsilon\}$. Let $U_{P}=\widetilde{U}_{P} \cap D_{\varepsilon} \times D_{\delta}{ }^{N}$ and $C_{P}=D_{\delta} \times 0 \subset U_{P} \cap C$. 
Since $C=\cup_{P} C_{P}$ and $C$ is compact, there are finitely many $C_{P}$ 's covering $C$. We use the notation $\widetilde{U}_{i}, U_{i}, \widetilde{C}_{i}, C_{i}, P_{i}(i=1, \ldots, r)$ for these (cf. (1b.9.1)). In $D_{\varepsilon} \times D_{\varepsilon}{ }^{N}$ containing $\widetilde{U}_{i}$, the closed set $\bar{C}_{i} \times\left(\bar{D}_{\delta}{ }^{N}-D_{\delta / 2}{ }^{N}\right)$ is compact and disjoint from $\widetilde{C}_{i}$. Hence in $\cup \widetilde{U}_{i}, \cup \bar{C}_{i} \times\left(\bar{D}_{\delta}^{N}-D_{\delta / 2}^{N}\right)$ is compact and disjoint from $C$. Thus there is an open neighborhood $V \subset \bigcup U_{i}$ of $C$ such that $\bar{V}$ is disjoint from $\cup \bar{C}_{i} \times\left(\bar{D}_{\delta}^{N}-D_{\delta / 2}^{N}\right)$. In particular $\left(\bar{V} \cap U_{i}\right) \cap \bar{C}_{i} \times D_{\delta}{ }^{N} \subset \bar{C}_{i} \times D_{\delta / 2}{ }^{N}$ for all $i$. Since $D_{\delta / 2}{ }^{N} \subset \subset D_{\delta}{ }^{N},\left(\bar{V} \cap U_{i}\right) \cap\left(\bar{C}_{i} \times D_{\delta}{ }^{N}\right)$ is compact for all $i$. We note that the first projections $f_{i}: U_{i} \rightarrow \widetilde{C}_{i}$ are not necessarily compatible with each other.

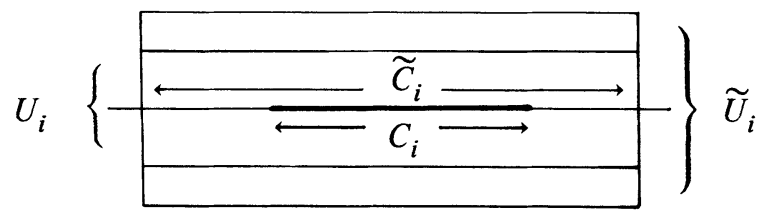

By replacing $V$ with a smaller open neighborhood of $C$, one may assume that $V \subset \cup f_{i}^{-1}\left(C_{i}\right)$, and that $E_{i}=f_{i}^{-1}\left(P_{i}\right) \cap V$ is closed in $V$ and hence an effective Cartier divisor. Let $E=E_{1}+\cdots+E_{r}$. Let $C^{\prime}$ be an irreducible compact subvariety in $V$. Then $\left(C^{\prime} \cap U_{i}\right) \cap f_{i}^{-1}\left(\bar{C}_{i}\right)$ is closed in the compact set $\left(\bar{V} \cap U_{i}\right) \cap\left(\bar{C}_{i} \times D_{\delta}{ }^{N}\right)$ hence is compact. Thus the induced map $f_{i}^{\prime}: C_{i}^{\prime} \rightarrow C_{i}$ is proper for all $i$, where $C_{i}^{\prime}=\left(C^{\prime} \cap U_{i}\right) \cap f_{i}^{-1}\left(C_{i}\right)$. Since fibers of $f_{i}^{\prime}$ lie in $D_{\delta / 2}{ }^{N}$ and compact, $f_{i}^{\prime}$ must be finite. Thus $\operatorname{dim} C^{\prime}=1$ and $\left(C^{\prime} \cdot E_{i}\right) \geq 0$ by $C^{\prime}=\bigcup C_{i}^{\prime}$ and $f_{i}^{\prime}$ is open and proper. Let $j$ be such that $C_{j}^{\prime} \neq \varnothing$. Then $f_{j}\left(C_{j}^{\prime}\right)=C_{j}$ by connectedness of $C_{j}$ and $\left(E_{j} \cdot C^{\prime}\right)=\left(f_{j}^{-1}\left(P_{j}\right) \cdot C^{\prime}\right)>0$. Hence $\left(E \cdot C^{\prime}\right) \geq\left(E_{j} \cdot C^{\prime}\right)>0$.

(1b.10) Corollary. Under the notation and assumptions of (1b.9), assume that there is a line bundle $L$ on $X$ such that $(L \cdot C)>0$. Then there is an open neighborhood $W$ of $C$ in $X$ such that every irreducible compact subvariety $C^{\prime}$ in $W$ with dimension $>0$ is a curve and $\left(L \cdot C^{\prime}\right)>0$.

Proof. Let $V$ and $E$ be as in (1b.9). By [Go, Théorème 4.11.1], one has

$$
\lim _{U \text { open } \supset C} H^{2}\left(U, \mathbb{Z}_{U}\right) \simeq H^{2}\left(C, \mathbb{Z}_{C}\right) \simeq \mathbb{Z}
$$

Thus on a sufficiently small neighborhood $W$ of $C,(L \cdot C) E_{W}$ and $L_{W} \otimes(E \cdot C)$ induce the same class in $H^{2}\left(W, \mathbb{Z}_{W}\right)$, where $E_{W}$ and $L_{W}$ are restrictions of $E$ and $L$ to $W$, respectively. Hence for $C^{\prime} \subset W$, one has $\left(L_{W} \cdot C^{\prime}\right)=\left(L \cdot C^{\prime}\right)>0$ from $\left(E_{W} \cdot C^{\prime}\right)=\left(E \cdot C^{\prime}\right)>0$. 
2. NUMERICAL INVARIANTS $i_{P}(n), w_{P}(0)$, AND $w_{P}^{*}(n)$

The main purpose of this section is to introduce numerical invariants which will be the main tools of our study.

We will use the results and definitions in $\S 1$ a on 3-fold terminal singularities.

(2.1) Let $X$ be an analytic normal Cohen-Macaulay 3-fold and $C \subset X$ a reduced curve such that $K_{X}$ is $\mathbb{Q}$-Cartier and no irreducible component of $C$ is contained in the singular locus of $X$.

(2.2) Definition. For coherent $\mathscr{O}_{X}$-modules $\mathscr{S} \supset \mathscr{T}$, we say that $\mathscr{T}$ is saturated in $\mathscr{S}$ (or, $\mathscr{S}$-saturated) iff $\mathscr{S} / \mathscr{T}$ has no $\mathscr{O}_{X}$-submodule of finite length $>0$. For each $\mathscr{T} \subset \mathscr{S}$, there is a smallest $\mathscr{S}$-saturated submodule $\mathscr{V}$ containing $\mathscr{T}$, and one sees that $\operatorname{Supp}(\mathscr{V} / \mathscr{T})$ is 0 -dimensional and $\operatorname{len}_{P} \mathscr{V} \mid \mathscr{T}<\infty$ at all $P \in X$. We call such $\mathscr{V}$ the $\mathscr{S}$-saturation of $\mathscr{T}$ and denote it by $\operatorname{Sat}_{\mathscr{S}} \mathscr{T}$.

Let $I_{C}$ (resp. $I_{C}^{(n)}$ ) be the sheaf of ideals defining $C$ in $X$ (resp. the symbolic $n$th power of $I_{C}$, that is $\left.\operatorname{Sat}_{\mathscr{O}} I_{C}{ }^{n}\right)$. For a coherent $\mathscr{O}_{X}$-module $\mathscr{S}$, let $F^{n} \mathscr{S}=$ $\operatorname{Sat}_{\mathscr{S}} I_{C}{ }^{n} \mathscr{S}=\operatorname{Sat}_{\mathscr{S}} I_{C}^{(n)} \mathscr{S}(n \geq 0)$ and $\operatorname{gr}_{C}^{n}(\mathscr{S})=F^{n} \mathscr{S} / F^{n+1} \mathscr{S}$. Let $\operatorname{gr}_{C}^{n} \mathscr{O}=$ $\operatorname{gr}_{C}^{n}\left(\mathscr{O}_{X}\right)$ and $\operatorname{gr}_{C}^{n} \omega=\operatorname{gr}_{C}^{n}\left(\omega_{X}\right)$. We note that $\mathrm{gr}^{*} \mathscr{O}=\bigoplus_{n \geq 0} \mathrm{gr}_{C}^{n} \mathscr{O}$ is naturally a graded $\mathscr{O}_{C}$-algebra and $\operatorname{gr}_{C}(\mathscr{S})=\bigoplus_{n \geq 0} \operatorname{gr}_{C}^{n}(\mathscr{S})$ is naturally a graded gr $\mathscr{O}$ module, and one has a natural homomorphism

$$
\gamma_{C}^{n}(\mathscr{S}): S^{n}\left(\mathrm{gr}_{C}^{1} \mathscr{O}\right) \otimes \operatorname{gr}_{C}^{0}(\mathscr{S}) \rightarrow \operatorname{gr}_{C}^{n}(\mathscr{S}) \quad(n \geq 1),
$$

where $S^{n}\left(\operatorname{gr}_{C}^{1} \mathscr{O}\right)$ denotes the symmetric $n$th power of $\operatorname{gr}_{C}^{1} \mathscr{O}$.

It is easy to see the following

(2.2.1) Lemma. Via the natural map $\Omega_{X}^{3} \rightarrow \omega_{X}$, there is a map

$$
\begin{gathered}
\operatorname{gr}_{C}^{1} \mathscr{O} \times \operatorname{gr}_{C}^{1} \mathscr{O} \times \Omega_{C}^{1} \rightarrow \Omega_{X}^{3} \otimes \mathscr{O}_{C} \rightarrow \operatorname{gr}_{C}^{0} \omega, \\
x \times y \times z d u \mapsto z d x \wedge d y \wedge d u
\end{gathered}
$$

which induces a homomorphism $\Lambda^{2}\left(\mathrm{gr}_{C}^{1} \mathscr{O}\right) \otimes \Omega_{C}^{1} \rightarrow \mathrm{gr}_{C}^{0} \omega$.

Thus one can define homomorphisms

$$
\begin{gathered}
\alpha_{1}: \bigwedge^{2}\left(\mathrm{gr}_{C}^{1} \mathscr{O}\right) \rightarrow \mathscr{H}_{m_{\mathscr{O}_{C}}}\left(\Omega_{C}^{1}, \operatorname{gr}_{C}^{0} \omega\right), \\
\alpha_{n}=\gamma_{C}^{n}\left(\mathscr{O}_{X}\right): S^{n}\left(\operatorname{gr}_{C}^{1} \mathscr{O}\right) \rightarrow \operatorname{gr}_{C}^{n} \mathscr{O} \quad(n \geq 2) .
\end{gathered}
$$

We denote by $i_{P}(n)$ the length $\operatorname{len}_{P} \operatorname{Coker} \alpha_{n}$ of $\operatorname{Coker} \alpha_{n}$ at $P \in C$. We note that $i_{P}(n) \in \mathbb{Z}_{+}(P \in C)$ and that $i_{P}(n)=0$ if $X$ and $C$ are smooth at $P$.

Let $m \in \mathbb{N}$ be such that $m K_{X}$ is a Cartier divisor, and let

$$
\begin{gathered}
\beta_{0}:\left(\operatorname{gr}_{C}^{0} \omega\right)^{\otimes m} \rightarrow \omega_{X}^{(m)} \otimes \mathscr{O}_{C}, \\
\beta_{n}=\gamma_{C}^{n}\left(\omega_{X}\right): \operatorname{gr}_{C}^{0} \omega \otimes S^{n}\left(\operatorname{gr}_{C}^{1} \mathscr{O}\right) \rightarrow \operatorname{gr}_{C}^{n} \omega \quad(n \geq 1)
\end{gathered}
$$


be the natural homomorphisms, where $\omega_{X}^{(m)}$ is the double dual of $\omega_{X}^{\otimes m}$.

We denote by $w_{P}(n)$ for $n \geq 1$ (resp. $\left.w_{P}(0)\right)$ len ${ }_{P}$ Coker $\beta_{n}$ (resp. $\left.\left(\operatorname{len}_{P} \operatorname{Coker} \beta_{0}\right) / m \in \mathbb{Q}\right)$, and define

$$
w_{P}^{*}(n)=\left(\begin{array}{c}
n+1 \\
2
\end{array}\right) i_{P}(1)-w_{P}(n) \text { for } n \geq 1 .
$$

We note that $w_{P}(n) \in \mathbb{Z}_{+}, w_{P}^{*}(n) \in \mathbb{Z} \quad(P \in C)$, and $w_{P}(n)=w_{P}^{*}(n)=0$ $(n \geq 1)$ if $X$ and $C$ are smooth at $P$. We also note that $w_{P}(0)$ is independent of the choice of $m$ if $C$ is smooth at $P$.

(2.3) From the above constructions, one can see

(2.3.1) (Formulae) If $C \simeq \mathbb{P}^{1}$, then

$$
\begin{aligned}
\operatorname{deg} \operatorname{gr}_{C}^{1} \mathscr{O} & =2+\operatorname{deg}_{C}^{0} \omega-\sum_{P} i_{P}(1), \\
\operatorname{deg} \operatorname{gr}_{C}^{n} \mathscr{O} & =\frac{1}{2} n(n+1) \operatorname{deg}_{C}^{1} \mathscr{O}+\sum_{P} i_{P}(n) \quad(n \geq 2), \\
\left(\omega_{X} \cdot C\right) & =\operatorname{deg} \operatorname{gr}_{C}^{0} \omega+\sum_{P} w_{P}(0), \quad \text { and } \\
\operatorname{deg} \operatorname{gr}_{C}^{n} \omega & =\frac{1}{2}(n+1)(n+2) \operatorname{deggr}_{C}^{0} \omega+n(n+1)-\sum_{P} w_{P}^{*}(n) \quad(n \geq 1) .
\end{aligned}
$$

(2.3.2) (Formulae) If $X \supset C \simeq \mathbb{P}^{1}$ is an extremal nbd, then $\operatorname{gr}_{C}^{0} \omega \simeq \mathscr{O}_{C}(-1)$, and

$$
\begin{aligned}
\operatorname{deg}_{C} \operatorname{gr}_{C}^{1} \mathscr{O} & =1-\sum_{P} i_{P}(1), \\
\operatorname{deg} \operatorname{gr}_{C}^{n} \mathscr{O} & =\frac{1}{2} n(n+1) \operatorname{deggr}_{C}^{1} \mathscr{O}+\sum_{P} i_{P}(n) \quad(n \geq 2), \\
\left(\omega_{X} \cdot C\right) & =-1+\sum_{P} w_{P}(0), \quad \text { and } \\
\operatorname{deg} \operatorname{gr}_{C}^{n} \omega & =\frac{1}{2}(n+1)(n-2)-\sum_{P} w_{P}^{*}(n) \quad(n \geq 1) .
\end{aligned}
$$

(2.3.3) (Necessary conditions) If $X \supset C \simeq \mathbb{P}^{1}$ is an extremal nbd, then

$$
\begin{aligned}
& \sum_{P} w_{P}(0)<1, \\
& \sum_{1 \leq i \leq n} \operatorname{deg} \operatorname{gr}_{C}^{i} \mathscr{O} \geq-\sum_{1 \leq i \leq n}(i+1) \quad \text { for all } n \in \mathbb{N}, \\
& \sum_{1 \leq i \leq n} \operatorname{deg}_{C}^{i} \omega \geq-\sum_{1 \leq i \leq n}(i+1) \quad \text { for all } n \in \mathbb{N},
\end{aligned}
$$

and when $n=1$, these reduce to

$$
\sum_{P} i_{P}(1) \leq 3 \text { and } \quad \sum_{P} w_{P}^{*}(1) \leq 1 .
$$


Proof of (2.3). Indeed (2.3.1) follows immediately from the constructions of $\alpha_{1}, \alpha_{n}, \beta_{0}$, and $\beta_{n}$. By $(1.14(\mathrm{i}))$, we have $\operatorname{gr}_{C}^{0} \omega \simeq \mathscr{O}_{C}(-1)$, hence the assertions in (2.3.2) follow. For (2.3.3), one has $H^{1}\left(\mathscr{O}_{X} / I_{C}^{(n+1)}\right)=H^{1}\left(\omega_{X} / F^{n+1} \omega_{X}\right)$ $=0$ by (1.2.1). Then $H^{1}\left(I_{C} / I_{C}^{(n+1)}\right)=0$ from the exact sequence

$$
0 \rightarrow I_{C} / I_{C}^{(n+1)} \rightarrow \mathscr{O}_{X} / I_{C}^{(n+1)} \rightarrow \mathscr{O}_{C} \rightarrow 0
$$

and $H^{0}\left(\mathscr{O}_{X} / I_{C}^{(n+1)}\right) \rightarrow H^{0}\left(\mathscr{O}_{C}\right)=\mathbb{C}$. Then from the exact sequences:

$$
\begin{aligned}
& 0 \rightarrow \mathrm{gr}_{C}^{n} \mathscr{O} \rightarrow I_{C} / I_{C}^{(n+1)} \rightarrow I_{C} / I_{C}^{(n)} \rightarrow 0 \quad(n \geq 1), \\
& 0 \rightarrow \mathrm{gr}_{C}^{n} \omega \rightarrow \omega_{X} / F^{n+1} \omega_{X} \rightarrow \omega_{X} / F^{n} \omega_{X} \rightarrow 0 \quad(n \geq 0),
\end{aligned}
$$

one has

$$
\chi\left(I_{C} / I_{C}^{(n+1)}\right)=\sum_{i=1}^{n} \chi\left(\mathrm{gr}_{C}^{i} \mathscr{O}\right) \text { and } \chi\left(\omega_{X} / F^{n+1} \omega_{X}\right)=\sum_{i=0}^{n} \chi\left(\mathrm{gr}_{C}^{i} \omega\right) .
$$

Now (2.3.3) follows from these.

(2.3.4) Remark. Let $n \in \mathbb{Z}_{+}$. The proof shows that if $\operatorname{deggr}_{C}^{d} \mathscr{O} \leq-d-$ 1 (resp. $\operatorname{deg}_{C}^{d} \omega \leq-d-1$ ) for $d \in[1, n]$, then $\operatorname{gr}_{C}^{d} \mathscr{O} \simeq \mathscr{O}_{C}(-1)^{\oplus(d+1)}$ (resp. $\operatorname{gr}_{C}^{d} \omega \simeq \mathscr{O}_{C}(-1)^{\oplus(d+1)}$ ) for $d \in[1, n]$ and $H^{1}\left(\mathrm{gr}_{C}^{n+1} \mathscr{O}\right)=0$ (resp. $\left.H^{1}\left(\operatorname{gr}_{C}^{n+1} \omega\right)=0\right)$.

(2.4) In the remainder of this section, we assume that $(X, P)$ is a germ of a 3-fold analytic terminal singularity of index $m$ and $C \subset(X, P)$ is a smooth curve such that $P$ has subindex $\bar{m}$ and splitting degree $s$ (1.7). We note that $m=\bar{m} \cdot s$. Let $\left(X^{\sharp}, P^{\sharp}\right) \rightarrow(X, P)$ be the canonical $\mu_{m}$-cover and $C^{\sharp}=$ $\left(C \times_{X} X^{\sharp}\right)_{\text {red }} \subset\left(X^{\sharp}, P^{\sharp}\right)$. Then $\left(C^{\sharp}, P^{\sharp}\right)$ has $s$ irreducible components (1.7), and $C^{\sharp}$ is not contained in the singular locus of $X^{\sharp}$.

(2.5) Definition. Let $\left(C^{\dagger}, P^{\dagger}\right)$ be an irreducible component of the normalization of one of the irreducible components $C^{\sharp}(i) \quad(i \in[1, s])$ of $\left(C^{\sharp}, P^{\sharp}\right)$. Then $\boldsymbol{\mu}_{m}$ naturally acts on $\left(X^{\sharp}, P^{\sharp}\right)$ and $\left(C^{\sharp}, P^{\sharp}\right)$, and so does $\boldsymbol{\mu}_{\dot{m}}$ on $\left(C^{\dagger}, P^{\dagger}\right)(1.7)$, and let

$$
\tau: \mathscr{O}_{X^{\sharp}, P^{\sharp}} \rightarrow \mathscr{O}_{C^{\dagger}, P^{\dagger}}
$$

be the natural map. Since $(X, P)$ and $(C, P)$ are normal, one has

$$
\mathscr{O}_{X, P}=\left(\mathscr{O}_{X^{\sharp}, P^{\sharp}}\right)^{\boldsymbol{\mu}_{m}} \text {, and } \mathscr{O}_{C, P}=\left(\mathscr{O}_{C^{\dagger}, P^{\dagger}}\right)^{\boldsymbol{\mu}_{\bar{m}}} \text {. }
$$

Since $\mu_{m}$ acts freely on $X^{\sharp}-\left\{P^{\sharp}\right\}$, so does it on $C^{\sharp}-\left\{P^{\sharp}\right\}$ and so does $\mu_{\bar{m}}$ on $C^{\dagger}-\left\{P^{\dagger}\right\}$. Hence $\mathscr{O}_{C^{\dagger}, P^{\dagger}}$ has a uniformizing parameter $t$ (i.e. $\mathscr{O}_{C^{\dagger}, P^{\dagger}}=\mathbb{C}\{t\}$ ) such that $t$ is a $\mu_{m}$-semi-invariant, and let $\chi$ be a generator of $X\left(\mu_{m}\right)=$ $\operatorname{Hom}\left(\boldsymbol{\mu}_{m}, \mathbb{C}^{*}\right)=\mathbb{Z}_{m}$ such that the restriction $\bar{\chi}$ of $\chi$ to $\mu_{\bar{m}}$ is the character associated to $t$. Hence $\mathscr{O}_{C, P}=\mathbb{C}\left\{t^{\bar{m}}\right\}$, and we call such $t$ an $\ell$-coordinate of 
$(C, P)$, and $\chi$ an $\ell$-character of $(C, P)$. We note that $\bar{\chi} \equiv \chi \bmod \bar{m} \mathbb{Z}_{m}$ is determined independent of choice of $\ell$-coordinate $t$.

For a semi-invariant $v \neq 0$, let $C^{\sharp}$-wt $v$ (or simply wt $v$ if there is no danger of confusion) be $n \in \mathbb{Z}_{m}$ such that $n \chi$ (or $\chi^{n}$ if written multiplicatively) is the character associated to $v$. We note that changing $\ell$-character $\chi$ to $a \chi \quad\left(a \in \mathbb{Z}_{m}\right.$ is a unit such that $a \equiv 1 \bmod \bar{m})$ changes $C^{\sharp}$-wt $v$ to $a^{-1} C^{\sharp}$-wt $v$.

If $C^{\dagger}$ dominates $C^{\sharp}(i)$, then for $z \in \mathscr{O}_{X^{\sharp}, P^{\sharp}}$, let

$$
C^{\sharp}(i)-\operatorname{ord}(z)=\sup \left\{n \in \mathbb{Z}_{+} \mid \tau(z) \in t^{n} \mathbb{C}\{t\}\right\} .
$$

If $z$ is a $\mu_{m}$-semi-invariant, then $C^{\sharp}(i)-\operatorname{ord}(z)=C^{\sharp}(j)-\operatorname{ord}(z)$ for all $i, j$, since $\mu_{m}$ acts transitively on $C^{\sharp}(i)$ 's. In this case, we may write $C^{\sharp}(i)$-ord $(z)$ as $C^{\sharp}$-ord $(z)$ or even as $\operatorname{ord}(z)$, if it does not cause confusion.

For a $\mu_{m}$-semi-invariant $z \in \mathscr{O}_{X^{\sharp}, P^{\sharp}}$, we define $C^{\sharp}$-ow $(z) \quad($ or ow $(z))$ as $\operatorname{ow}(z)=(\operatorname{ord}(z), \mathrm{wt}(z)) \in\left(\mathbb{Z}_{+} \cup\{\infty\}\right) \times \mathbb{Z}_{m}$. We note that $\operatorname{ord}(z) \equiv \mathrm{wt}(z)$ $(\bmod \bar{m})$ if $\operatorname{ord}(z)<\infty$. We define semigroups

$$
\begin{aligned}
\operatorname{ord}\left(C^{\sharp}\right) & =\left\{\operatorname{ord}(w) \mid w \in \mathscr{O}_{C^{\sharp}, P^{\sharp}}-I_{C^{\sharp}, P^{\sharp}}\right\} \subset \mathbb{Z}_{+}, \quad \text { and } \\
\operatorname{ow}\left(C^{\sharp}\right) & =\left\{\operatorname{ow}(w) \mid w \in \mathscr{O}_{C^{\sharp}, P^{\sharp}}-I_{C^{\sharp}, P^{\sharp}}\right\} \subset \mathbb{Z}_{+} \times \mathbb{Z}_{m} .
\end{aligned}
$$

Assume that $\boldsymbol{\mu}_{m}$ acts on a $\mathbb{C}$-algebra $R$ via $\mathbb{C}$-algebra automorphisms, and that $M$ is a $\mu_{m}-R$-module. Let $n \in \mathbb{Z}_{m}$. Let $\mathbb{C}[n]$ be the 1-dimensional $\mathbb{C}$-vector space on which $\boldsymbol{\mu}_{m}$ acts by $n \chi$, where $\chi$ is an $\ell$-character of $(C, P)$, and let $M[n]=M \otimes_{\mathbb{C}} \mathbb{C}[n]$ be the $R$-module with the naturally induced $\boldsymbol{\mu}_{m}$-action. Let

$$
\begin{aligned}
M_{\{n\}} & =\operatorname{Hom}_{\boldsymbol{\mu}_{m}}(\mathbb{C}[n], M) \\
& =\left\{x \in M \mid \gamma(x)=\chi(\gamma)^{n} x \text { for all } \gamma \in \boldsymbol{\mu}_{m}\right\} .
\end{aligned}
$$

Then $M_{\{n\}}=M[-n]_{\{0\}}$ is an $R_{\{0\}}$-module.

(2.6) Definition. Let $(x)$ be an $\ell$-coordinate system for $(X, P)$ (1a.5). We note that $\mathrm{wt}(x)$ up to permutation does not depend on choice of $(x)$. Let $a_{i}=\operatorname{ord}\left(x_{i}\right) \quad\left(i \in[1,4], 0<a_{i} \leq \infty\right)$. We say that $(x)$ is minimal if $a_{i}$ is the smallest positive integer such that $\left(a_{i}\right.$, wt $\left.x_{i}\right) \in \operatorname{ow}\left(C^{\sharp}\right)$ (in particular $\left.a_{i}<\infty\right)$ for all $i$. Thus $\operatorname{ord}(x)$ and $\operatorname{ow}(x)$ up to permutation do not depend on choice of minimal $(x)$. We say that $(x)$ and an $\ell$-coordinate $t$ of $(C, P)$ are normalized $\ell$-coordinates if $(x)$ is minimal and $\tau\left(x_{i}\right)=t^{a_{i}}$ for all $i$ (cf. $(2.7$ (iv))). Then we have

(2.7) Lemma. Let $(X, P) \supset C$ be as in (2.4), and $\chi$ an $\ell$-character. Then

(i) if $(x)$ and $t$ are an $\ell$-coordinate system of $(X, P)$ and an $\ell$-coordinate of $(C, P)$, then there exists an $\ell$-coordinate system $(y)$ of $(X, P)$ such that

$$
\mathrm{ow}\left(y_{i}\right)=\mathrm{ow}\left(x_{i}\right) \text { and } \tau\left(y_{i}\right)=t^{a_{i}} \text { for all } i,
$$


(ii) for $(y)$ and $t$ as in (i), let $\Lambda=\left\{i \mid \operatorname{ord}\left(y_{i}\right) \neq \infty\right\}$. Then $y_{i}(i \in \Lambda)$ give an embedding $\left(C^{\sharp}, P^{\sharp}\right) \subset\left(\mathbb{C}^{\Lambda}, 0\right)$ such that the images of $s$ irreducible components are parametrized by $t$ as

$$
\left(\chi(g)^{\operatorname{wt}\left(y_{i}\right)} t^{\operatorname{ord}\left(y_{i}\right)}\right)_{i \in \Lambda} \quad\left(g \in \boldsymbol{\mu}_{m} / \boldsymbol{\mu}_{\bar{m}} \simeq \boldsymbol{\mu}_{s}\right),
$$

ow induces an isomorphism of semigroups

$$
\mathrm{ow}_{y}:\left\{\begin{array}{l}
\text { functions } \neq 0 \text { on }\left(C^{\sharp}, P^{\sharp}\right) \text { which are } \\
\text { monomials in } y \text { with coefficients } 1
\end{array}\right\} \stackrel{\rightarrow}{\mathrm{ow}}\left(C^{\sharp}\right),
$$

(iii) $(\bar{m}, 0) \in \operatorname{ow}\left(C^{\sharp}\right)$, and $(u, v) \in \operatorname{ow}\left(C^{\sharp}\right)$ for all large enough $u \in \mathbb{N}$ and all $v \in \mathbb{Z}_{m}$ such that $u \equiv v(\bmod \bar{m})$, and

(iv) $(X, P) \supset C$ have normalized $\ell$-coordinates.

Proof. Since $\tau$ is $\mu_{m}$-equivariant, we have $\tau\left(x_{i}\right)=t^{a_{i}} g_{i}$ for some units $g_{i} \in$ $\mathbb{C}\left\{t^{\bar{m}}\right\}$. Since

$$
\operatorname{Im}\left(\mathscr{O}_{X, P} \rightarrow \mathscr{O}_{C^{\dagger}, P^{\dagger}}\right)=\operatorname{Im}\left(\mathscr{O}_{C, P} \rightarrow \mathscr{O}_{C^{\dagger}, P^{\dagger}}\right)=\mathbb{C}\left\{t^{\bar{m}}\right\},
$$

we can choose units $h_{i} \in \mathscr{O}_{X, P}$ such that $\tau\left(h_{i}\right)=g_{i}$ for all $i$. Then it is enough to take $y_{i}=x_{i} h_{i}^{-1}$ for (i). Since $\mu_{m}$ acts transitively on irreducible components of $C^{\sharp}$, the first assertion of (ii) follows easily. Whence ow ${ }_{y}$ is injective, and we see that, for each $\boldsymbol{\mu}_{m}$-semi-invariant $u$, there exists a monomial $v$ in $y$ such that $\operatorname{ow}(u)=\mathrm{ow}(v)$. Hence follows (ii). As for (iii), $(\bar{m}, 0) \in \mathrm{ow}\left(C^{\sharp}\right)$ follows from $\mathscr{O}_{C, P} \stackrel{\sim}{\longrightarrow} \mathbb{C}\left\{t^{\bar{m}}\right\}$, and we see (wt $\left.y_{i}, m\right)=1$ for some $i \in \Lambda$ given in (ii) because the $\mu_{m}$-action on $C^{\sharp}-\left\{P^{\sharp}\right\}$ is free. Whence the second assertion follows because $\mathbb{Z}_{+}(\bar{m}, 0)+\mathbb{Z}_{+}$ow $\left(y_{i}\right)$ contains $(u, v)$ given in (iii). For (iv), let $(x)$ and $t$ be as in (i). For each $i$, let $f_{i} \in \mathbb{C}\{x\}$ be a $\mu_{m}$-semi-invariant with $\operatorname{wt}\left(f_{i}\right) \equiv \mathrm{wt}\left(x_{i}\right) \quad(m)$, minimizing $\operatorname{ord}\left(f_{i}\right)$. For some suitable $\varepsilon \in \mathbb{C}$, $\left(x^{\sharp}\right)=\left(x_{1}+\varepsilon f_{1}, \ldots, x_{4}+\varepsilon f_{4}\right)$ is an $\ell$-coordinate system such that $\operatorname{ord}\left(x^{\sharp}\right)$ is the smallest. Then (i) gives normalized $\ell$-coordinates.

(2.8) Definition. Under the notation and assumptions of (2.4), let $z=(x, w) \in$ $\mathbb{Z}_{+} \times \mathbb{Z}_{m}$ be such that $x \equiv w(\bmod \bar{m})$.

(i) We define $U(z), R(w) \in \mathbb{Z}$ as

$$
\begin{aligned}
U(z) & =\min \left\{k \in \mathbb{Z} \mid k(\bar{m}, 0)-z \in \operatorname{ow}\left(C^{\sharp}\right)\right\} \\
& =1+\max \left\{k \in \mathbb{Z} \mid k(\bar{m}, 0)-z \notin \operatorname{ow}\left(C^{\sharp}\right)\right\}, \quad \text { and } \\
R(w) & =\min \left\{u \in \mathbb{Z}_{+} \mid(u,-w) \in \operatorname{ow}\left(C^{\sharp}\right)\right\} .
\end{aligned}
$$

We may write $R(w)$ as $R(z)$. These are well defined since second projection ow $\left(C^{\sharp}\right) \rightarrow \mathbb{Z}_{m}$ is surjective and the equality for $U(z)$ follows by $(\bar{m}, 0) \in$ ow $\left(C^{\sharp}\right)(2.7(\mathrm{iii}))$. For a $\mu_{m}$-semi-invariant $v$, we denote $(R($ wt $v),-$ wt $v) \in$ ow $\left(C^{\sharp}\right)$ by $C^{\sharp}$ ow $^{*}(v)\left(\right.$ or ow ow $\left.^{*}(v)\right)$, 
(ii) we see $\bar{m} \cdot U(z)=x+R(z)$ and $U(z+(\bar{m}, 0))=U(z)+1$. We may write $U(x, x \bmod m)$ as $U(x)$ for $x \in \mathbb{Z}$. In particular,

(iii) if $P$ is a primitive point, then $s=1$ and $\bar{m}=m$, whence $U(z)$ and $R(z)$ reduce to

$$
\begin{aligned}
U(x) & =\min \left\{k \in \mathbb{Z} \mid m k-x \in \operatorname{ord}\left(C^{\sharp}\right)\right\} \\
& =1+\max \left\{k \in \mathbb{Z} \mid m k-x \notin \operatorname{ord}\left(C^{\sharp}\right)\right\}, \\
R(x) & =R(x \bmod m)=\min \left\{u \in \operatorname{ord}\left(C^{\sharp}\right) \mid u \equiv-x(m)\right\},
\end{aligned}
$$

with the properties $m \cdot U(x)=x+R(x), U(x+m)=U(x)+1$.

(2.9) Lemma. One has

$$
I_{C}^{(n)}=\left(I_{C^{\sharp}}^{(n)}\right)_{\{0\}} \quad \text { and } \quad F^{n} \omega_{X}=\left(F^{n} \omega_{X^{\sharp}}\right)_{\{0\}},
$$

whence

$$
\operatorname{gr}_{C}^{n}\left(\mathscr{O}_{X}\right)=\operatorname{gr}_{C^{\sharp}}^{n}\left(\mathscr{O}_{X^{\sharp}}\right)_{\{0\}} \text { and } \operatorname{gr}_{C}^{n}\left(\omega_{X}\right)=\operatorname{gr}_{C^{\sharp}}^{n}\left(\omega_{X^{\sharp}}\right)_{\{0\}} \text {. }
$$

Proof. Since $\left(I_{C^{\sharp}}^{(n)}\right)_{\{0\}}=\mathscr{O}_{X, P} \cap I_{C^{\sharp}}^{(n)}$, one sees that $\left(I_{C^{\sharp}}^{(n)}\right)_{\{0\}}$ is a primary ideal associated to $C$. Since $X^{\sharp}$ is étale over $X$ at general points of $C^{\sharp}, I_{C}^{(n)}=$ $\left(I_{C^{\sharp}}^{(n)}\right)_{\{0\}}$ at general points of $C^{\sharp}$, whence $I_{C}^{(n)}=\left(I_{C^{\sharp}}^{(n)}\right)_{\{0\}}$. Since $\omega_{X}=\left(\omega_{X^{\sharp}}\right)_{\{0\}}$ (via the trace map), the exact sequence

$$
0 \rightarrow F^{n} \omega_{X^{\sharp}} \rightarrow \omega_{X^{\sharp}} \rightarrow \operatorname{gr}_{C^{\sharp}}^{n} \omega_{X^{\sharp}} \rightarrow 0
$$

gives the exact sequence

$$
0 \rightarrow\left(F^{n} \omega_{X^{\sharp}}\right)_{\{0\}} \rightarrow \omega_{X} \rightarrow\left(\operatorname{gr}_{C^{\sharp}}^{n} \omega_{X^{\sharp}}\right)_{\{0\}} \rightarrow 0 .
$$

Since $X^{\sharp}$ is étale over $X$ at general points of $C^{\sharp}$, one has $I_{C}^{(n)} \omega_{X}=\left(F^{n} \omega_{X^{\sharp}}\right)_{\{0\}}$ at general points of $C$. Since $\omega_{X} /\left(F^{n} \omega_{X^{\sharp}}\right)_{\{0\}}$ is torsion-free, one has $F^{n} \omega_{X}=$ $\left(F^{n} \omega_{X^{\sharp}}\right)_{\{0\}}$.

Let $x$ be an $\ell$-coordinate system and $\phi$ an $\ell$-equation (1a.5).

(2.10) Corollary. One has $\omega_{X^{\sharp}} \otimes \mathscr{O}_{C^{\sharp}} \simeq \mathscr{O}_{C^{\sharp}}[k]$, and $w_{P}(0)=R(k) / \bar{m}$, where $k=C^{\sharp}-\mathrm{wt}\left(x_{1} x_{2} x_{3} x_{4} / \phi\right)$.

Proof. By the residue formula, $\omega_{X^{\sharp}} \otimes \mathscr{O}_{C^{\sharp}}$ is an invertible $\mathscr{O}_{C^{\sharp}, P^{\sharp}}$-module whose generator is the image $\bar{\omega}$ of

$$
\operatorname{Res}_{X^{\sharp}} \frac{d x_{1} \wedge d x_{2} \wedge d x_{3} \wedge d x_{4}}{\phi}
$$

which has $C^{\sharp}$-wt $\equiv k$, whence $\omega_{X^{\sharp}} \otimes \mathscr{O}_{C^{\sharp}} \simeq \mathscr{O}_{C^{\sharp}}[k]$. We see that $\operatorname{gr}_{C}^{0}(\omega)=$ $u \mathbb{C}\left\{t^{\bar{m}}\right\} \bar{\omega}$ for some semi-invariant $u \in \mathscr{O}_{X^{\sharp}, P^{\sharp}}$ with the minimal ord $u$ under the condition that wt $u+k \equiv 0(m)$. Hence $w_{P}(0)=R(k) / \bar{m}$ by definition of $R(k)$. 
(2.11) We introduce the following construction to compute $i_{P}(1)$ and $w_{P}^{*}(1)$ for the terminal singularity $(X, P)$ in (2.4).

Let $x$ and $\phi$ be an $\ell$-coordinate system and an $\ell$-equation at $(X, P)$ (1a.5), and $t$ an $\ell$-coordinate of $(C, P)(2.5)$. We will use the symbols introduced in (2.5) and (2.6). Let $h_{i}(t)=\tau\left(x_{i}\right) \in \mathbb{C}\{t\}$ and $\phi_{i}=\partial \phi / \partial x_{i} \quad(i \in[1,4])$. By symmetry among $x_{1}, \ldots, x_{4}$, we will assume that $h_{4}(t) \neq 0$.

Let $\left(X^{\sharp}, P^{\sharp}\right) \subset\left(Z^{\sharp}, P^{\sharp}\right)=\left(x_{1} x_{2} x_{3} x_{4}\right.$-space, 0$)$ be an embedding by the $\ell$ coordinate system $x$. Let $\left(C^{\dagger}, P^{\dagger}\right) \subset\left(Z^{\dagger}, P^{\dagger}\right)=\left(y_{1} y_{2} y_{3} y_{4}\right.$-space, 0$)$ be the embedding $(t) \mapsto\left(h_{1}(t), h_{2}(t), h_{3}(t), t\right)$. Then $(y) \mapsto\left(y_{1}, y_{2}, y_{3}, h_{4}\left(y_{4}\right)\right)$ defines a morphism $\left(Z^{\dagger}, P^{\dagger}\right) \mapsto\left(Z^{\sharp}, P^{\sharp}\right)$ fitting in the commutative diagram

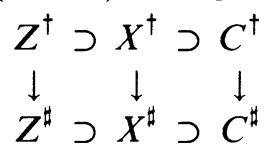

where $X^{\dagger} \subset Z^{\dagger}$ is defined by $\psi(y) \equiv \phi\left(y_{1}, y_{2}, y_{3}, h_{4}\left(y_{4}\right)\right)=0$.

We note that $\mu_{\bar{m}}$ naturally acts on $Z^{\dagger}, X^{\dagger}, C^{\dagger}$, and that $C^{\dagger}$ is not contained in the singular locus of $X^{\dagger}$ because $X^{\dagger} \rightarrow X^{\sharp}$ is étale outside $\left\{y_{4}=0\right\}$ and $C^{\dagger}=\left\{\bar{y}_{1}=\bar{y}_{2}=\bar{y}_{3}=0\right\}$, where $\bar{y}_{i}=y_{i}-h_{i}\left(y_{4}\right)$ for $i=1,2,3$.

Let $J^{\sharp}$ (resp. $J^{\dagger}$ ) be the ideal defining $C^{\sharp}$ in $Z^{\sharp}$ (resp. $C^{\dagger}$ in $Z^{\dagger}$ ). Let $J^{\sharp(n)}$ and $J^{\dagger(n)}$ be the symbolic $n$th power of $J^{\sharp}$ and $J^{\dagger}$, respectively. Then $J^{\dagger}=\left(\bar{y}_{1}, \bar{y}_{2}, \bar{y}_{3}\right)$, and

$$
J^{\dagger} / J^{\dagger(2)} \simeq \mathbb{C}\{t\} \bar{y}_{1} \oplus \mathbb{C}\{t\} \bar{y}_{2} \oplus \mathbb{C}\{t\} \bar{y}_{3}
$$

(we identify $y_{4}=t$ in $\mathscr{O}_{C^{\dagger}, P^{\dagger}}$ ) and let

$$
\sigma: J^{\sharp} \rightarrow J^{\dagger} / J^{\dagger(2)}
$$

be the natural map. For $g_{1}, g_{2}, g_{3} \in J^{\dagger} / J^{\dagger(2)}$, let $\left[g_{1}, g_{2}, g_{3}\right] \in \mathbb{Z}_{+} \cup\{\infty\}$ be the length of

$$
\left(J^{\dagger} / J^{\dagger(2)}\right) / \mathbb{C}\{t\} g_{1}+\mathbb{C}\{t\} g_{2}+\mathbb{C}\{t\} g_{3},
$$

and for subsets $A, B, C \subset J^{\dagger} / J^{\dagger(2)}$, let

$$
[A, B, C]=\inf \{[a, b, c] \mid a \in A, b \in B, c \in C\} .
$$

By abuse of notation, one may denote $[\ldots, \sigma a, \ldots]$ by $[\ldots, a, \ldots]$ when $a \in$ $J^{\sharp}\left(\operatorname{resp} .[\ldots, \sigma A, \ldots]\right.$ by $[\ldots, A, \ldots]$ when $\left.A \subset J^{\sharp}\right)$. We note that $[*, *, *]$ has semiadditivity

$$
\left[g_{1}, g_{2}, g_{3}+g_{3}^{\prime}\right] \geq \min \left\{\left[g_{1}, g_{2}, g_{3}\right],\left[g_{1}, g_{2}, g_{3}^{\prime}\right]\right\}
$$

because $\left[g_{1}, g_{2}, g_{3}\right]=$ ord $g_{1} \wedge g_{2} \wedge g_{3}$.

(2.12) Proposition. Under the notation of (2.11), one has

$$
\begin{aligned}
i_{P}(1) & =\bar{m}^{-1} \cdot\left(\bar{m}-R(k)-\operatorname{ord}\left(x_{4}\right)+\left[\phi, J_{\{0\}}^{\sharp}, J_{\{0\}}^{\sharp}\right]\right) \quad \text { and } \\
w_{P}^{*}(1) & =\bar{m}^{-1} \cdot\left(\bar{m}-3 R(k)-\operatorname{ord}\left(x_{4}\right)+\left[\phi, J_{\{-k\}}^{\sharp}, J_{\{-k\}}^{\sharp}\right]\right)
\end{aligned}
$$

if $\operatorname{ord}\left(x_{4}\right)<\infty$, where $k=C^{\sharp}-\mathrm{wt}\left(x_{1} x_{2} x_{3} x_{4} / \phi\right)$. 
Proof. By symmetry among $x_{1}, x_{2}, x_{3}$, one may assume

$$
\tau\left(\phi_{2}\right), \tau\left(\phi_{3}\right) \in \tau\left(\phi_{1}\right) \mathbb{C}\{t\} \text {. }
$$

In this proof, we denote tensor products over $\mathscr{O}_{X}\left(\right.$ resp. $\left.\mathscr{O}_{X^{\sharp}}, \mathscr{O}_{X^{\dagger}}\right)$ by $\otimes($ resp. $\left.\otimes^{\sharp}, \otimes^{\dagger}\right)$.

We put $\alpha_{1}$ and $\beta_{1}$ in the commutative diagrams of natural homomorphisms

$$
\begin{array}{cc}
\left(\bigwedge^{2} \operatorname{gr}_{C}^{1} \mathscr{O}\right) \otimes \omega_{C} \otimes \mathscr{O}_{C^{\dagger}} \stackrel{\alpha_{1} \otimes \mathscr{O}_{C^{\dagger}}}{\longrightarrow} \operatorname{gr}_{C}^{0} \omega \otimes \mathscr{O}_{C^{\dagger}} & \downarrow f_{3} \\
\downarrow\left(\bigwedge^{2} f_{1}\right) \otimes^{\dagger} f_{2} & \\
\left(\bigwedge^{2} \operatorname{gr}_{C^{\dagger}}^{1} \mathscr{O}\right) \otimes^{\dagger} \omega_{C^{\dagger}} \stackrel{f_{4}}{\longrightarrow} \omega_{X^{\dagger}} \otimes^{\dagger} \mathscr{O}_{C^{\dagger}}
\end{array}
$$

and

$$
\begin{array}{cc}
\left(\mathrm{gr}_{C}^{0} \omega\right) \otimes\left(\mathrm{gr}_{C}^{1} \mathscr{O}\right) \otimes \mathscr{O}_{C^{\dagger}} \stackrel{\beta_{1} \otimes \mathscr{O}_{C^{\dagger}}}{\longrightarrow} & \left(\mathrm{gr}_{C}^{1} \omega\right) \otimes \mathscr{O}_{C^{\dagger}} \\
\downarrow^{g_{2} \otimes^{\dagger} f_{1}} & \downarrow g_{1} \\
\omega_{X^{\sharp}} \otimes^{\sharp} \operatorname{gr}_{C^{\dagger}}^{1} \mathscr{O} & \stackrel{\sim}{\longrightarrow} \operatorname{gr}_{C^{\dagger}}^{1}\left(\omega_{X^{\sharp}} \otimes^{\sharp} \mathscr{O}_{C^{\dagger}}\right)
\end{array}
$$

where

$$
\begin{aligned}
& f_{1}: \operatorname{gr}_{C}^{1} \mathscr{O} \otimes \mathscr{O}_{C^{\dagger}} \rightarrow \operatorname{gr}_{C^{\dagger}}^{1} \mathscr{O}, \\
& f_{2}: \omega_{C} \otimes \mathscr{O}_{C^{\dagger}} \rightarrow \omega_{C^{\dagger}}, \\
& g_{2}:\left(\operatorname{gr}_{C}^{0} \omega\right) \otimes \mathscr{O}_{C^{\dagger}} \rightarrow \omega_{X^{\sharp}} \otimes \mathscr{O}_{C^{\dagger}},
\end{aligned}
$$

and $f_{4}$ is defined similarly to $\alpha_{1}$. Thus

$$
\bar{m} \cdot i_{P}(1)=\sum_{i=1,2,4} \text { len Coker } f_{i}-\operatorname{len} \operatorname{Coker} f_{3},
$$

and

$$
\bar{m} \cdot w_{P}(1)=2 \cdot \operatorname{len} \text { Coker } g_{2}+\operatorname{len} \text { Coker } f_{1}-\text { len Coker } g_{1} \text {, }
$$

because $\left(C^{\dagger}, P^{\dagger}\right) \rightarrow(C, P)$ is of degree $\bar{m}$. We note

$$
\begin{aligned}
\sigma(\phi) & =\phi\left(y_{1}, y_{2}, y_{3}, h_{4}\left(y_{4}\right)\right) \bmod J^{\dagger(2)} \\
& =\phi\left(h_{1}+\bar{y}_{1}, h_{2}+\bar{y}_{2}, h_{3}+\bar{y}_{3}, h_{4}\right) \bmod J^{\dagger(2)} \\
& =\tau\left(\phi_{1}\right) \bar{y}_{1}+\tau\left(\phi_{2}\right) \bar{y}_{2}+\tau\left(\phi_{3}\right) \bar{y}_{3} .
\end{aligned}
$$

Since $C^{\sharp} \not \subset$ Sing $X^{\sharp}(2.4)$, one has $\sigma(\phi) \neq 0$. Hence $\tau\left(\phi_{1}\right) \neq 0$, and $\sigma(\phi) / \tau\left(\phi_{1}\right)$ is a part of a free basis of $J^{\dagger} / J^{\dagger(2)}$. Then we claim

$$
\operatorname{gr}_{C^{\dagger}}^{1}\left(\mathscr{O}_{X^{\dagger}}\right) \simeq\left(J^{\dagger} / J^{\dagger(2)}\right) / \mathbb{C}\{t\} \sigma(\phi) / \tau\left(\phi_{1}\right) .
$$

Indeed, since $\sigma(\phi)=\left(\sigma(\phi) / \tau\left(\phi_{1}\right)\right) \cdot \tau\left(\phi_{1}\right) \in J^{\dagger} / J^{\dagger(2)}$ is sent to 0 in $\operatorname{gr}_{C^{\dagger}}^{1}\left(\mathscr{O}_{X^{\dagger}}\right)$ and $\tau\left(\phi_{1}\right) \neq 0$ in $\mathscr{O}_{C^{+}}$, one sees that $\sigma(\phi) / \tau\left(\phi_{1}\right)$ is also sent to 0 . Thus it induces a surjective homomorphism

$$
\left(J^{\dagger} / J^{\dagger(2)}\right) / \mathbb{C}\{t\} \sigma(\phi) / \tau\left(\phi_{1}\right) \rightarrow \operatorname{gr}_{C^{\dagger}}^{1}\left(\mathscr{O}_{X^{\dagger}}\right),
$$


which is an isomorphism because both are free of rank 2 , and (2.12.1) is checked. Since we have $\mathscr{O}_{C}$-module surjections

$$
\operatorname{gr}_{C}^{1} \mathscr{O} \simeq\left(\operatorname{gr}_{C^{\sharp}}^{1} \mathscr{O}\right)_{\{0\}} \nleftarrow J_{\{0\}}^{\sharp}
$$

and

$$
\operatorname{gr}_{C}^{1} \omega \simeq\left(\operatorname{gr}_{C^{\sharp}}^{1} \mathscr{O} \otimes^{\sharp} \omega_{X^{\sharp}}\right)_{\{0\}} \simeq\left(\operatorname{gr}_{C^{\sharp}}^{1} \mathscr{O}\right)_{\{-k\}} \nleftarrow J_{\{-k\}}^{\sharp}
$$

by (2.9) and (2.10), we can use (2.12.1) to calculate the cokernels of $f_{1}$ and $g_{1}$ as

$$
\text { Coker } f_{1} \simeq\left(J^{\dagger} / J^{\dagger(2)}\right) / \mathbb{C}\{t\} \sigma\left(J_{\{0\}}^{\sharp}\right)+\mathbb{C}\{t\}\left(\sigma(\phi) / \tau\left(\phi_{1}\right)\right),
$$

and

$$
\text { Coker } g_{1} \simeq\left(J^{\dagger} / J^{\dagger(2)}\right) / \mathbb{C}\{t\} \sigma\left(J_{\{-k\}}^{\sharp}\right)+\mathbb{C}\{t\}\left(\sigma(\phi) / \tau\left(\phi_{1}\right)\right) .
$$

Hence we have

$$
\operatorname{len}\left(\operatorname{Coker} f_{1}\right)=\left[\phi, J_{\{0\}}^{\sharp}, J_{\{0\}}^{\sharp}\right]-\operatorname{ord} \tau\left(\phi_{1}\right),
$$

and

$$
\operatorname{len}\left(\text { Coker } g_{1}\right)=\left[\phi, J_{\{-k\}}^{\sharp}, J_{\{-k\}}^{\sharp}\right]-\text { ord } \tau\left(\phi_{1}\right),
$$

because $\sigma(\phi) / \tau\left(\phi_{1}\right)$ is a part of a free basis of $J^{\dagger} / J^{\dagger(2)}$.

It is clear that len Coker $f_{2}=\bar{m}-1$, because $C^{\dagger} \rightarrow C$ is given by $t \mapsto t^{\bar{m}}$. One sees that the cokernel of

$$
f_{3}: \operatorname{gr}_{C}^{0} \omega \otimes \mathscr{O}_{C^{\dagger}} \rightarrow \omega_{X^{\sharp}} \otimes^{\sharp} \mathscr{O}_{C^{\dagger}} \rightarrow \omega_{X^{\dagger}} \otimes^{\dagger} \mathscr{O}_{C^{\dagger}}
$$

has length $R(k)+a_{4}-1$ because the cokernel of $g_{2}: \operatorname{gr}_{C}^{0} \omega \otimes \mathscr{O}_{C^{\dagger}} \rightarrow \omega_{X^{\sharp}} \otimes^{\sharp} \mathscr{O}_{C^{\dagger}}$ has length $R(k)$ by the proof of $(2.10)$ and

$$
\begin{aligned}
\omega_{X^{\sharp}} \otimes^{\sharp} \mathscr{O}_{C^{\dagger}} & =\mathscr{O}_{C^{\dagger}} \frac{d x_{2} \wedge d x_{3} \wedge d x_{4}}{\phi_{1}} \\
& =t^{a_{4}-1} \mathscr{O}_{C^{\dagger}} \frac{d \bar{y}_{2} \wedge d \bar{y}_{3} \wedge d y_{4}}{\psi_{1}}=t^{a_{4}-1} \omega_{X^{\dagger}} \otimes^{\dagger} \mathscr{O}_{C^{\dagger}} .
\end{aligned}
$$

One sees that len Coker $f_{4}=\operatorname{ord}\left(\tau \phi_{1}\right)$ because we have

$$
\operatorname{Im} f_{4}=\mathscr{O}_{C^{\dagger}} d \bar{y}_{2} \wedge d \bar{y}_{3} \wedge d y_{4}=\psi_{1} \omega_{X^{\dagger}} \otimes^{\dagger} \mathscr{O}_{C^{\dagger}}=\tau \phi_{1} \omega_{X^{\dagger}} \otimes^{\dagger} \mathscr{O}_{C^{\dagger}}
$$

by $\operatorname{gr}_{C^{+}}^{1} \mathscr{O}=\mathscr{O}_{C^{+}} \bar{y}_{2} \oplus \mathscr{O}_{C^{+}} \bar{y}_{3}$ (2.12.1). Thus one has

$$
\bar{m} \cdot i_{P}(1)=\bar{m}-R(k)-a_{4}+\left[\phi, J_{\{0\}}^{\sharp}, J_{\{0\}}^{\sharp}\right] .
$$

Since len Coker $g_{2}=R(k)$ as above, one also has

$$
\bar{m} \cdot w_{P}(1)=2 \cdot R(k)+\left[\phi, J_{\{0\}}^{\sharp}, J_{\{0\}}^{\sharp}\right]-\left[\phi, J_{\{-k\}}^{\sharp}, J_{\{-k\}}^{\sharp}\right],
$$

whence

$$
\bar{m} \cdot w_{P}^{*}(1)=\bar{m}-3 \cdot R(k)-a_{4}+\left[\phi, J_{\{-k\}}^{\sharp}, J_{\{-k\}}^{\sharp}\right] .
$$


(2.13) Definition. For $b \in \mathbb{Z}_{+}^{4}$, let

$$
x^{b}=x_{1}^{b_{1}} x_{2}^{b_{2}} x_{3}^{b_{3}} x_{4}^{b_{4}}, \quad \text { where } b=\left(b_{1}, b_{2}, b_{3}, b_{4}\right) .
$$

For normalized $\ell$-coordinates $(x)$ and $t$, we define

(2.13.1) for $b, c \in \mathbb{Z}_{+}{ }^{4}$ such that $\operatorname{ow}\left(x^{b}\right)=\operatorname{ow}\left(x^{c}\right)$, let $P(b, c)=x^{b}-$ $x^{c},\|P(b, c)\|=\operatorname{ord}\left(x^{b}\right) \in \mathbb{Z}_{+}$if $b \neq c \quad(\infty$ if $b=c)$, and let us define $\operatorname{ex}_{4}(P(b, c)) \in \mathbb{Z}^{3}$ by $\operatorname{ex}_{4}(P(b, c))_{i}=b_{i}-c_{i}(i \in[1,3])$. Then all such $P(b, c)$ generate the ideal $J^{\sharp}$, and

$$
P(b, c)=P(b, d)-P(c, d),
$$

$$
P\left(b+b^{\prime}, c+c^{\prime}\right)=P(b, c) x^{b^{\prime}}-P\left(b^{\prime}, c^{\prime}\right) x^{c}
$$

if $b, c, d, b^{\prime}, c^{\prime} \in \mathbb{Z}_{+}^{4}$ satisfy $\operatorname{ow}\left(x^{b}\right)=\operatorname{ow}\left(x^{c}\right)=\operatorname{ow}\left(x^{d}\right)$ and ow $\left(x^{b^{\prime}}\right)=$ ow $\left(x^{c^{\prime}}\right)$.

The following allows us to calculate $[*, *, *]$ in (2.11).

(2.14) Proposition. Assume that $(x)$ and $t$ are normalized and let us use the notation of (2.11). Then for $P$ as in (2.13.1), one has

$$
\sigma(P)=t^{\|P\|} \sum_{i=1}^{3} \operatorname{ex}_{4}(P)_{i} Y_{i} \quad\left(Y_{i}=\bar{y}_{i} / t^{a_{i}}\right)
$$

and hence

$$
\left[P_{1}, P_{2}, P_{3}\right]=\left\{\begin{array}{l}
\infty \text { if } \operatorname{ex}_{4}\left(P_{1}\right), \operatorname{ex}_{4}\left(P_{2}\right), \mathrm{ex}_{4}\left(P_{3}\right) \text { are dependent } \\
\sum_{i=1,2,3}\left\|P_{i}\right\|-\sum_{i=1,2,3} a_{i} \text { otherwise }
\end{array}\right.
$$

for such $P_{1}, P_{2}, P_{3}$ as in (2.13.1).

Proof. One sees

$$
\begin{aligned}
\sigma(P) & \equiv t^{a_{4} b_{4}} \prod_{i=1}^{3}\left(t^{a_{i}}+\bar{y}_{i}\right)^{b_{i}}-t^{a_{4} c_{4}} \prod_{i=1}^{3}\left(t^{a_{i}}+\bar{v}_{i}\right)^{c_{i}} \quad\left(J^{\dagger(2)}\right) \\
& \equiv t^{\|P\|}\left(\prod_{i=1}^{3}\left(1+Y_{i}\right)^{b_{i}}-\prod_{i=1}^{3}\left(1+Y_{i}\right)^{c_{i}}\right) \\
& \equiv t^{\|P\|} \sum_{i=1}^{3}\left(b_{i}-c_{i}\right) Y_{i}=t^{\|P\|} \sum_{i=1}^{3} \operatorname{ex}_{4}(P)_{i} Y_{i}, \quad \square
\end{aligned}
$$

(2.15) Corollary. Under the notation and assumptions of (2.4), assume that $(X, P)$ is singular. Then $i_{P}(1) \geq 1$.

Proof. If $m=1$, then $C^{\sharp} \simeq C$ is smooth and the assertion follows from (2.16). Let us assume $m>1$ and choose normalized $\ell$-coordinates $(x)$ and $t(2.7)$ 
and an $\ell$-equation $\phi(1 \mathrm{a} .5)$ so that wt $x_{4} \equiv \mathrm{wt} \phi$ and wt $x_{1} x_{2} \equiv 0(\mathrm{~m})$ by the classification of terminal singularities (1a.5), and we will follow (2.11). By (1a.6), we see that wt $x_{1}$, wt $x_{2}$, wt $x_{3}$ are prime to $m$, and treat two cases.

Case where $C^{\sharp}-\mathrm{wt} \phi \equiv 0(m)$. One has $a_{4}=\bar{m}$ since $(x)$ and $t$ are normalized and $(\bar{m}, 0) \in \operatorname{ow}\left(C^{\sharp}\right)$. One has

$$
\bar{m} \cdot i_{P}(1) \geq\left[J_{\{0\}}^{\sharp}, J_{\{0\}}^{\sharp}, J_{\{0\}}^{\sharp}\right]-R\left(\text { wt } x_{3}\right)
$$

by (2.12). Hence we see $\bar{m} \cdot i_{p}(1) \geq\left[P_{1}, P_{2}, P_{3}\right]-R\left(\right.$ wt $\left.x_{3}\right)$ for suitable invariant $P_{1}, P_{2}, P_{3}$ in $(2.13 .1)$. Hence $\operatorname{wt}\left(P_{i}\right) \equiv 0(\bmod m)$ for all $i$. Since $\operatorname{ex}_{4}\left(P_{1}\right), \ldots, \operatorname{ex}_{4}\left(P_{3}\right)$ are independent, one sees that there is a permutation $\gamma \in \mathfrak{S}_{3}$ such that $\operatorname{ex}_{4}\left(P_{\gamma(i)}\right)_{i} \neq 0$ for all $i \in[1,3]$ by expanding the determinant. Thus one has monomials $v_{i}$ in $x$ such that ow $\left(v_{i} x_{i}\right)=\left(\left\|P_{\gamma(i)}\right\|, 0\right)$, and we have $v_{i} \neq 1$ by wt $x_{i} \not \equiv 0(m)$ for all $i \in[1,3]$. Hence $\left\|P_{\gamma(i)}\right\|>a_{i}$ for $i=1,2$ and $\left\|P_{\gamma(3)}\right\| \geq a_{3}+R\left(\right.$ wt $\left.x_{3}\right)$ (2.8). By (2.14), one has

$$
\bar{m} \cdot i_{P}(1) \geq \sum_{i=1,2}\left\{\left\|P_{\gamma(i)}\right\|-a_{i}\right\}+\left\|P_{\gamma(3)}\right\|-a_{3}-R\left(\text { wt } x_{3}\right)>0 \text {. }
$$

Case where $m=4$ and $C^{\sharp}$-wt $\phi \equiv 2$ (4). Then $(4,0) \in \mathrm{ow}\left(C^{\sharp}\right)$ by $(2.7(\mathrm{iii}))$, and $(4,0) \in\left(\operatorname{ow}\left(C^{\sharp}\right)-\{0\}\right)+\left(\operatorname{ow}\left(C^{\sharp}\right)-\{0\}\right)$ by $(2.7(\mathrm{ii}))$ because no $x_{i}$ has wt $\equiv 0$ (4), whence $(1, \pm 1),(2,0)$, or $(2,2) \in \mathrm{ow}\left(C^{\sharp}\right)$ by $\bar{m}=2,4$ (1.16). If $(2,0) \in \operatorname{ow}\left(C^{\sharp}\right)$, then we can repeat the same argument to get $(1, \pm 1) \in \operatorname{ow}\left(C^{\sharp}\right)$. If $(1, \pm 1) \in \operatorname{ow}\left(C^{\sharp}\right)$, then we have $(2,2)=2(1, \pm 1) \in \operatorname{ow}\left(C^{\sharp}\right)$. Thus we have $(2,2) \in \operatorname{ow}\left(C^{\sharp}\right)$ anyway, and hence $a_{4}=\operatorname{ord} x_{4}=2$ by normalizedness. Since $x_{4} \phi \in J_{\{0\}}^{\sharp},(2.12)$ implies the same formula as (2.15.1):

$$
\begin{aligned}
m \cdot i_{P}(1) & \geq m-R\left(\text { wt } x_{3}\right)-a_{4}+\left[J_{\{0\}}^{\sharp}, J_{\{0\}}^{\sharp}, J_{\{0\}}^{\sharp}\right]-a_{4} \\
& =\left[J_{\{0\}}^{\sharp}, J_{\{0\}}^{\sharp}, J_{\{0\}}^{\sharp}\right]-R\left(\text { wt } x_{3}\right),
\end{aligned}
$$

where we used that $m=4=2 a_{4}$. The rest is the same as the previous case.

(2.16) Lemma. Assume that $\left(C^{\sharp}, P^{\sharp}\right)$ is smno l-coordinate system $(x)$ and assumptions of (2.4). Then there exists notation and assumptions of (2.4). Then there exists an l-coordinate system $(x)$ and an $\ell$-equation $\phi$ (1a.5) of $(X, P)$ such that $C^{\sharp}$ is the $x_{4}$-axis and $\phi \equiv x_{4}{ }^{r} x_{1} \bmod \left(x_{1}, x_{2}, x_{3}\right)^{2} \quad(r \geq 0)$. Furthermore we have

(i) $\operatorname{len}_{P^{\sharp}} I_{C^{\sharp}}^{(2)} / I_{C^{\sharp}}{ }^{2}=r$,

(ii) if wt $\phi \equiv 0 \quad(m)$ then

$$
i_{P}(1)= \begin{cases}r & \text { if } m=1 \\ {[r / m]+1} & \text { if } m>1\end{cases}
$$

and

(iii) if $m=4$ and wt $\phi \equiv 2$ (4) then $i_{P}(1)=[(r+6) / 4]$. 
Proof. We note that $s=1$ and $\bar{m}=m$ by smoothness of $C^{\sharp}$. The first assertion is obvious, and (i) follows from $I_{C^{\sharp}}{ }^{(2)} / I_{C^{\sharp}}{ }^{2} \simeq\left(\mathscr{O}_{C^{\sharp}} /\left(x_{4}{ }^{r}\right)\right) \cdot x_{1} \simeq$ $\mathbb{C}\left\{x_{4}\right\} /\left(x_{4}^{r}\right)$. From $(2.12)$ and the definition of $[*, *, *]$ follows

$$
\begin{aligned}
i_{P}(1) & =\frac{1}{m}\left\{m-R\left(\sum_{i=1}^{4} \mathrm{wt} x_{i}-\mathrm{wt} \phi\right)-1+\left[x_{4}^{r} x_{1}, x_{4}^{R\left(\mathrm{wt} x_{2}\right)} x_{2}, x_{4}^{R\left(\mathrm{wt} x_{3}\right)} x_{3}\right]\right\} \\
& =\frac{1}{m}\left\{\sum_{i=1}^{4} R\left(\mathrm{wt} x_{i}\right)+r-R\left(\mathrm{wt} x_{1}\right)-R\left(\sum_{i=1}^{4} \mathrm{wt} x_{i}-\mathrm{wt} \phi\right)\right\} .
\end{aligned}
$$

If $m=1$, then we are done because $R(z)=0$ for $z \in \mathbb{Z}$. Assume that $m>1$ and wt $\phi=0(m)$. By (1a.6), there exists $\sigma \in \mathfrak{S}_{4}$ such that $R\left(\right.$ wt $\left.x_{\sigma(1)}\right)=0$, $R\left(\right.$ wt $\left.x_{\sigma(2)}\right)+R\left(\right.$ wt $\left.x_{\sigma(3)}\right)=m$, whence

$$
\sum_{1 \leq i \leq 4} R\left(\text { wt } x_{i}\right)-R\left(\sum_{1 \leq i \leq 4} \mathrm{wt} x_{i}-\mathrm{wt} \phi\right)=m,
$$

and

$$
i_{P}(1)=\frac{1}{m}\left\{m+r-R\left(\text { wt } x_{1}\right)\right\}=[r / m]+1,
$$

because $R\left(\right.$ wt $\left.x_{1}\right) \in[0, m)$. This proves (ii). Assume that $m=4$ and wt $\phi \equiv 2$ (4). By (1a.6), there exists $\sigma \in \mathfrak{S}_{4}$ such that $R\left(\right.$ wt $\left.x_{\sigma(1)}\right)=2, R\left(\right.$ wt $\left.x_{\sigma(2)}\right)+$ $R\left(\right.$ wt $\left.x_{\sigma(3)}\right)=4$, whence $\sum_{1 \leq i \leq 4} R\left(\right.$ wt $\left.x_{i}\right)-R\left(\sum_{1 \leq i \leq 4}\right.$ wt $x_{i}-$ wt $\left.\phi\right)=6$, and

$$
i_{P}(1)=\frac{1}{4}\left\{r+6-R\left(\text { wt } x_{1}\right)\right\}=[(r+6) / 4] \text {, }
$$

because $R\left(\right.$ wt $\left.x_{1}\right) \in[0,4)$.

\section{EMBEDDING Dimension of $\left(C^{\sharp}, P^{\sharp}\right)$}

The main purpose of this section is to prove that the pull back $C^{\sharp}(2.4)$ of $C$ by the canonical cover $\left(X^{\sharp}, P^{\sharp}\right) \rightarrow(X, P)$ has only planar singularities if $X \supset C \simeq \mathbb{P}^{1}$ is an extremal nbd. We treat primitive points and imprimitive points separately.

Part I. Primitive point $P$. The following is the main theorem.

(3.1) Theorem. If $X \supset C \simeq \mathbb{P}^{1}$ is an extremal nbd and $P$ a primitive point of index $m$, then emb $\operatorname{dim}_{P^{\sharp}} C^{\sharp} \leq 2$, where $\left(C^{\sharp}, P^{\sharp}\right)$ is the pull back of $C$ to the canonical cover $\left(X^{\sharp}, P^{\sharp}\right)$.

(3.2) Under the above notation and assumptions, let us choose normalized $\ell$-coordinates $(x)$ and $t(2.7)$, and an $\ell$-equation $\phi$ so that $C^{\sharp}$-wt $\phi \equiv$ $C^{\sharp}$-wt $x_{4}, C^{\sharp}$-wt $x_{2} x_{3} \equiv 0(m) \quad(1 \mathrm{a} .5)$. (We note that we may permute $x_{2}$ and $\left.x_{3}.\right)$

We note that emb $\operatorname{dim}_{P^{\sharp}} C^{\sharp} \leq 2$ is equivalent to "the semigroup $\operatorname{ord}\left(C^{\sharp}\right)$ is generated by two elements (2.7)." It is clear that ord $\left(C^{\sharp}\right)$ is generated by 2 and 
the smallest odd number if $2 \in \operatorname{ord}\left(C^{\sharp}\right)$. So we assume that $m, a_{1}, \ldots, a_{4} \geq 3$ and use the notation of $\S 2$ in Part I of this section.

First we treat the exceptional terminal singularities (1a.6).

(3.3) Lemma. If $m=4$ and $C^{\sharp}-\mathrm{wt}(\phi) \equiv 2$ (4), then one has $\operatorname{emb} \operatorname{dim}_{P^{\sharp}} C^{\sharp}$ $\leq 2$.

Proof. One has $a_{4} \equiv 2$ (4). Then $a_{1}, a_{2}, a_{3}, a_{4} \not \equiv 0$ (4) and

$$
4 \in\left(\operatorname{ord}\left(C^{\sharp}\right)-\{0\}\right)+\left(\operatorname{ord}\left(C^{\sharp}\right)-\{0\}\right)
$$

Whence $2 \in \operatorname{ord}\left(C^{\sharp}\right)$, and we are done by the remark in (3.2).

(3.4) Lemma. Assume that $w_{P}(0)<1, i_{P}(1) \leq 3$, and emb $\operatorname{dim}_{P \sharp} C^{\sharp} \geq 3$. Then $i_{P}(1)=3, a_{1}>m, a_{2}=3, a_{4}=m, R\left(a_{1}\right)=3 n$ for some integer $n \in[1, m / 3)$ modulo permutation of $x_{2}$ and $x_{3}$.

Proof. By normalizedness of $(x)$ and $t$, one has $a_{4}=m$ and $\left(a_{1} a_{2} a_{3}, m\right)=$ 1. Since $m \cdot w_{P}(0)=R\left(a_{1}\right)$ (2.10), one has $R\left(a_{1}\right)<m$ by (2.3.3). From $R\left(a_{1}\right) \in \operatorname{ord}\left(C^{\sharp}\right)$, one has $R\left(a_{1}\right) \in \mathbb{Z}_{+} a_{1}+\mathbb{Z}_{+} a_{\alpha} \quad(\alpha=2$ or 3$)$, because $a_{2}+a_{3}$, $a_{4} \equiv 0 \quad(m)$.

(3.4.1) We claim that $a_{1}>m$ and thus $R\left(a_{1}\right) \in \mathbb{Z} a_{\alpha}$. To see this, let us assume $a_{1} \leq m$. Then $a_{1}<m$ by $\left(a_{1}, m\right)=1$. Thus $m=a_{1}+R\left(a_{1}\right)$ by $R\left(a_{1}\right)$, $a_{1}<m$, and $a_{1}+R\left(a_{1}\right) \equiv 0(m)$. Whence $m \in \mathbb{Z}_{++} a_{1}+\mathbb{Z}_{+} a_{\alpha}$. Since $a_{1}>1$ and $\left(m, a_{1}\right)=1$, one sees $m \notin \mathbb{Z}_{+} a_{1}$ and hence $a_{4}=m \in \mathbb{Z}_{++} a_{1}+\mathbb{Z}_{++} a_{\alpha}$. Thus $a_{2}+a_{3} \in \mathbb{Z}_{++} a_{1}+\mathbb{Z}_{++} a_{\alpha}$ by $a_{2}+a_{3} \equiv 0(m)$, whence $a_{5-\alpha} \in \mathbb{Z}_{++} a_{1}+\mathbb{Z}_{+} a_{\alpha}$. Thus $a_{1}$ and $a_{\alpha}$ generate $\operatorname{ord}\left(C^{\sharp}\right)$, which is a contradiction, and (3.4.1) is proved.

(3.4.2) We claim that $m \geq 4$, and that $a_{2}$ or $a_{3}=3$ implies (3.4) if $i_{P}(1)=3$. First by a remark in (3.2), $m>R\left(a_{1}\right) \geq 3$, which implies $m \geq 4$. If one has $a_{3} \geq a_{2}=3$ modulo permutation, then $2 a_{3} \geq a_{2}+a_{3} \geq m$, whence $R\left(a_{1}\right)=a_{3}$ $(\alpha=3)$ or $R\left(a_{1}\right) \in 3 \mathbb{Z}_{+}(\alpha=2)$. If $R\left(a_{1}\right)=a_{3}$, then $a_{3}<m$. Thus one sees $a_{3}=m-3, a_{1} \equiv 3(m)$, and hence $a_{1}, a_{4} \in \mathbb{Z}_{+} a_{2}+\mathbb{Z}_{+} a_{3}$. This is a contradiction, and $R\left(a_{1}\right) \in 3 \mathbb{Z}_{+}$. Thus (3.4.2) is proved.

(3.4.3) Let $S$ be the set of $b=\left(b_{1}, b_{2}, b_{3}\right) \in \mathbb{Z}_{+}^{3}-\{0\}$ such that $a_{1} b_{1}+$ $a_{2} b_{2}+a_{3} b_{3} \equiv 0(\mathrm{~m})$. We say that $b \in S$ is reduced if $b \notin S+S$. Let $\|b\|=a_{1} b_{1}+a_{2} b_{2}+a_{3} b_{3}$.

Let $\lambda=(0,1,1)$. We note that $b=\lambda$ if $b \in S$ is reduced and satisfies $b_{2}, b_{3}>0$. For $b=\left(b_{1}, b_{2}, b_{3}\right) \in S$, let

$$
Q(b)=P\left(\left(b_{1}, b_{2}, b_{3}, 0\right),(0,0,0,\|b\| / m)\right),
$$

with the notation of (2.13.1). Then $\operatorname{ex}_{4}(Q(b))=b$ and $\|Q(b)\|=\|b\|$. One sees that $J_{\{0\}}^{\sharp}$ is generated by $Q(b) \quad(b \in S)$ by (2.13.2) and that by $Q(b)$ 's such that $b \in S$ is reduced because (2.13.3) reduces to

$$
Q\left(b+b^{\prime}\right)=Q(b) x^{b^{\prime}}-Q\left(b^{\prime}\right) x_{4}^{\|b\| / m} \quad\left(b, b^{\prime} \in S\right) .
$$


By subadditivity of $[*, *, *](2.11),(2.12)$ implies

$$
\begin{aligned}
3 m \geq m \cdot i_{P}(1) & =-R\left(a_{1}\right)+\left[\phi, J_{\{0\}}^{\sharp}, J_{\{0\}}^{\sharp}\right] \\
& \geq-R\left(a_{1}\right)+\left[Q\left(b^{1}\right), Q\left(b^{2}\right), Q\left(b^{3}\right)\right]
\end{aligned}
$$

for some reduced $b^{1}, b^{2}, b^{3} \in S$. Throughout Part I of this section, $b^{i}$ denotes such a vector not a power of $b$. By (2.14), one has

$$
3 m \geq \sum_{i=1}^{3}\left(\left\|b^{i}\right\|-a_{i}\right)-R\left(a_{1}\right)
$$

and that $b^{1}, b^{2}, b^{3}$ are independent. Let $b^{i}=\left(b_{j}^{i}\right)$. We note

$$
\left\|b^{i}\right\| \geq\left(b_{1}^{i}-1\right) m+a_{1}+R\left(a_{1}\right) \quad \text { if } b_{1}^{i} \geq 1 .
$$

Indeed one sees $\left\|b^{i}\right\| \geq\left(b_{1}^{i}-1\right) a_{1}+a_{1} \geq\left(b_{1}^{i}-1\right) m+a_{1}$ by (3.4.1), whence $\left\|b^{i}\right\|-\left(b_{1}^{i}-1\right) m \geq a_{1}$. Thus $\left\|b^{i}\right\|-\left(b_{1}^{i}-1\right) m \geq a_{1}+R\left(a_{1}\right)$ because $\left\|b^{i}\right\| \equiv 0$ ( $m)$ and $a_{1}+R\left(a_{1}\right)=m \cdot\left\lceil a_{1} / m\right\rceil$ by $R\left(a_{1}\right)<m$.

(3.4.6) We treat three cases.

$$
\left(\begin{array}{ccc}
+ & & 0 \\
+ & 0 \\
0 & +
\end{array}\right) \quad\left(\begin{array}{lll}
+ & & 0 \\
+ & & \\
0 & 1 & 1
\end{array}\right) \quad\left(\begin{array}{ccc}
+ & & \\
0 & + & 0 \\
0 & 1 & 1
\end{array}\right) \quad \begin{gathered}
b^{i} \text { is the } i \text { th row. } \\
+ \text { at }(i, j) \text { means }
\end{gathered}
$$

Figure (3.4.A) Figure (3.4.B) Figure (3.4.C)

Case (A). None of $b^{1}, b^{2}, b^{3}$ is $\lambda$. We will derive a contradiction in this case. Expanding $\operatorname{det}\left(b_{j}^{i}\right)$, one sees that there is a $\sigma \in \mathfrak{S}_{3}$ such that $b_{i}^{\sigma(i)} \neq 0$ for all $i$. Since $b^{\sigma(1)} \neq \lambda$, one has $b_{\tau(3)}^{\sigma(1)}=0$ for some $\tau \in \mathfrak{S}_{3}$ fixing 1 (3.4.3).

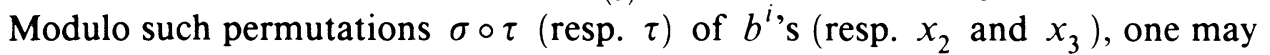
assume that $b_{i}^{i} \neq 0$ for all $i$ and $b_{3}^{1}=0$. Since $b^{2}$ and $b^{3}$ are reduced and $b^{2}, b^{3} \neq \lambda$, one sees that $b_{3}^{2}=b_{2}^{3}=0(3.4 .3)$. Now $\left(b_{j}^{i}\right)$ is as in Figure (3.4.A) above.

By (3.4.5), we rewrite (3.4.4) as

$$
\begin{aligned}
3 m & \geq\left(\left\|b^{1}\right\|-a_{1}-R\left(a_{1}\right)\right)+\left(\left\|b^{2}\right\|-a_{2}\right)+\left(\left\|b^{3}\right\|-a_{3}\right) \\
& \geq\left(b_{1}^{1}-1\right) m+\left(\left\|b^{2}\right\|-a_{2}\right)+\left(\left\|b^{3}\right\|-a_{3}\right) .
\end{aligned}
$$

Let $i \in[2,3]$. If $b_{1}^{i}=0$, then $b_{i}^{i}=m$ by $b_{3}^{2}=b_{2}^{3}=0$, whence $\left\|b^{i}\right\|-$ $a_{i}=(m-1) a_{i} \geq 3(m-1)>2 m$ by $a_{i} \geq 3$ and $m \geq 4$. If $b_{1}^{i} \geq 1$, then $\left\|b^{i}\right\|-a_{i} \geq b_{1}^{i} a_{1}>b_{1}^{i} m$. Hence from (3.4.6.1), one has $b_{1}^{2}=b_{1}^{3}=1$. Then (3.4.6.1) implies $3 m>\left(b_{1}^{1}-1\right) m+2 m$, and one has $b_{1}^{1}=1$. Since $b^{1}$ and $b^{2}$ are reduced and $b_{1}^{1}=b_{1}^{2}=1$, one has $b_{2}^{1}, b_{2}^{2} \in[0, m-1]$. By $b^{1}, b^{2} \in S$ and $b_{1}^{1}=b_{1}^{2}=1$ and $b_{3}^{1}=b_{3}^{2}=0$, one has $b_{2}^{1} \equiv b_{2}^{2}(m)$, whence $b_{2}^{1}=b_{2}^{2}$ and $b^{1}=b^{2}$, whence $b^{1}, b^{2}, b^{3}$ are dependent. This is a contradiction. 
Case (B). $b^{3}=\lambda$ and $b_{1}^{1}, b_{1}^{2}>0$ modulo permutation of $b^{1}, b^{2}, b^{3}$. We will also derive a contradiction in this case. Modulo permutation of $b^{1}$ and $b^{2}$ one may assume $\left\|b^{1}\right\| \leq\left\|b^{2}\right\|$, and modulo further permutation of $x_{2}$ and $x_{3}$ one may also assume $b_{3}^{1}=0$ because $b^{1}$ is reduced and $b^{1} \neq \lambda$ (3.4.3). Now $\left(b_{j}^{i}\right)$ is as in (3.4.B).

Since $a_{1}>m$, one has $2 m \leq a_{1}+R\left(a_{1}\right)$, whence $m \geq 3 m-a_{1}-R\left(a_{1}\right)$. Then (3.4.4) gives

$$
m \geq 3 m-\left(a_{1}+R\left(a_{1}\right)\right) \geq \sum_{i=1,2}\left\{\left\|b^{i}\right\|-\left(a_{1}+R\left(a_{1}\right)\right)\right\} .
$$

Since $\left\|b^{i}\right\|-\left(a_{1}+R\left(a_{1}\right)\right) \in m \mathbb{Z}_{+}$(3.4.5), one has $\left\|b^{1}\right\|=a_{1}+R\left(a_{1}\right)$ by $\left\|b^{1}\right\| \leq$ $\left\|b^{2}\right\|$. Then the inequality gives $\left\|b^{2}\right\| \leq 3 m$. Hence one has $b_{1}^{1}=1, b_{1}^{2}=1,2$ by (3.4.5). Since $b_{1}^{1}=1$ and $b_{3}^{1}=0$, one has $b_{2}^{1}>0$. Since $\left\|b^{1}\right\|=a_{1}+R\left(a_{1}\right)$, one has $b_{2}^{1}=R\left(a_{1}\right) / a_{2}<m / 3$. Since $b_{1}^{2}=1$ or 2 and since $b^{2}$ is reduced, one easily sees $b^{2}=\left(b_{1}^{2}, b_{1}^{2} \cdot b_{2}^{1}, 0\right)$ or $\left(b_{1}^{2}, 0, m-b_{1}^{2} \cdot b_{2}^{1}\right)$ by $b_{2}^{2} \cdot b_{3}^{2}=0$ and $0<b_{1}^{2} \cdot b_{2}^{1}<m$. We have the second case since $b^{2}$ is independent of $b^{1}$. Then

$$
\begin{aligned}
\left\|b^{2}\right\| & =b_{1}^{2} \cdot a_{1}+\left(m-b_{1}^{2} \cdot b_{2}^{1}\right) a_{3} \geq b_{1}^{2} \cdot a_{1}+3\left(m-b_{1}^{2} \cdot b_{2}^{1}\right) \\
& =3 m+b_{1}^{2}\left(a_{1}-3 b_{2}^{1}\right)>3 m,
\end{aligned}
$$

because $a_{1}>m>3 b_{2}^{1}$. This contradicts $\left\|b^{2}\right\| \leq 3 m$ obtained above.

Case (C). $b^{3}=\lambda, b_{1}^{1}>0$, and $b_{1}^{2}=0$ modulo permutation of $b^{1}, b^{2}, b^{3}$. Modulo permutation of $x_{2}$ and $x_{3}$, one may further assume $b_{3}^{2}=0$. Now $\left(b_{j}^{i}\right)$ is as in (3.4.C). Then $b^{2}=(0, m, 0)$ since it is reduced. By (3.4.5) applied to $b^{1}$ and (3.4.4), one has $3 m \geq\left(b_{1}^{1}-1\right) m+a_{2} m$. Thus $a_{2}=3$ and $i_{P}(1)=3$ from the inequality. Thus the proof of (3.4) is finished.

(3.5) We now prove (3.1). We will derive a contradiction assuming that there is a point $P \in C$ at which emb $\operatorname{dim}_{P \sharp} C^{\sharp} \geq 3$. By (2.3.3), one sees $w_{P}(0)<1$ and $i_{P}(1) \leq 3$, since $w_{Q}(0) \geq 0$ and $i_{Q}(1) \geq 0$ for all $Q \in C$. By (3.4), one has $i_{P}(1)=3$. One has $\sum_{Q \neq P} i_{Q}(1)=0$ by (2.3.3), whence $P$ is the only singular point of $X$ on $C$ by (2.15). Thus by (2.3.3), one has $w_{P}^{*}(1) \leq 1$. We will work at $P$ using the notation of (3.2), and estimate $w_{P}^{*}(1)$ by an argument similar to (3.4). By (3.4), one has $a_{2}=3, a_{4}=m, R\left(a_{1}\right)=3 n$ for some $n \in[1, m / 3)$, and $a_{1}>m$. By $x_{2}{ }^{n} \phi \in J_{\{3 n\}}^{\sharp}$, one sees from (2.12) that

$$
\begin{aligned}
m \geq m \cdot w_{P}^{*}(1) & =-9 n+\left[\phi, J_{\{3 n\}}^{\sharp}, J_{\{3 n\}}^{\sharp}\right] \\
& =-12 n+\left[x_{2}^{n} \phi, J_{\{3 n\}}^{\sharp}, J_{\{3 n\}}^{\sharp}\right] \\
& \geq\left[J_{\{3 n\}}^{\sharp}, J_{\{3 n\}}^{\sharp}, J_{\{3 n\}}^{\sharp}\right]-12 n .
\end{aligned}
$$

Let $\nu=(0, n, 0)$ and, following (3.4.3), let $T$ be the set of $b \in \mathbb{Z}_{+}{ }^{3}-\{\nu\}$ such that $\|b\| \equiv 3 n(m)$. We say that $b \in T$ is reduced if $b \notin T+S$ (3.4.3). 
For $b=\left(b_{1}, b_{2}, b_{3}\right) \in T$, let

$$
\bar{Q}(b)=P\left(\left(b_{1}, b_{2}, b_{3}, 0\right),(0, n, 0,(\|b\|-3 n) / m)\right) .
$$

Then $\operatorname{ex}_{4}(\bar{Q}(b))=b-\nu$ and $\|\bar{Q}(b)\|=\|b\|$. One sees that $J_{\{3 n\}}^{\sharp}$ is generated by $\bar{Q}(b) \quad(b \in T)$ by $(2.13 .2)$ because $3 n<m$, and that by $\bar{Q}(b)$ 's such that $b \in T$ is reduced, because (2.13.3) reduces to

$$
\bar{Q}\left(b+b^{\prime}\right)=\bar{Q}(b) x^{b^{\prime}}-\bar{Q}\left(b^{\prime}+\nu\right) x_{4}^{(\|b\|-3 n) / m} \quad\left(b \in T, b^{\prime} \in S\right) .
$$

By subadditivity of $[*, *, *](2.11),(2.14)$ and (3.5.1) imply

$$
m \geq \sum_{i=1}^{3}\left(\left\|b^{i}\right\|-a_{i}\right)-12 n
$$

for some reduced $b^{1}, b^{2}, b^{3} \in T$ such that $b^{1}-\nu, b^{2}-\nu, b^{3}-\nu$ are independent. Let $b^{i}=\left(b_{j}^{i}\right)$. As in case (A) of (3.4.6), we may assume $\left(b^{i}-\nu\right)_{i} \neq 0$ for all $i$.

(3.5.3) We note that an integer $z \in(0, m)$ belongs to ord $\left(C^{\sharp}\right)$ iff $z=a_{3}=m-3$ or $z \in 3 \mathbb{Z}_{+}$, and there is only one way to express such $z$ as sum of $a_{1}, a_{2}$, $a_{3}, a_{4}$.

(3.5.4) We claim $\left\|b^{1}\right\| \geq a_{1}+6 n$. One sees $6 n-m \notin \operatorname{ord}\left(C^{\sharp}\right)$ by (3.5.3), because $6 n-m<m$ by $m>3 n, 6 n-m \neq 0(3)$ by $(3, m)=1$, and $6 n-m \neq m-3$. On the other hand, one sees that $\left\|b^{1}\right\|-a_{1} \equiv 6 n-m(m)$ by $\left\|b^{1}\right\| \equiv 3 n \quad(m)$, and that $\left\|b^{1}\right\|-a_{1} \in \operatorname{ord}\left(C^{\sharp}\right)$ by $\left(b^{1}-\nu\right)_{1}=b_{1}^{1}>0$. Thus $\left\|b^{1}\right\|-a_{1} \geq 6 n$ (cf. (2.8)), which is (3.5.4).

(3.5.5) We claim $\left\|b^{3}\right\| \geq a_{3}+3+3 n$ and that the equality holds only when $b^{3}=(0, n+1,1)$. Let us see that $a_{1} z_{1}+a_{2} z_{2}+a_{3} z_{3}=3+3 n\left(z \in \mathbb{Z}_{+}{ }^{3}\right)$ has a unique solution $z=(0, n+1,0)$. We claim

$$
3+3 n \neq a_{1}, i a_{3} \quad(i=1,2) \text {. }
$$

Indeed $3 n+3=a_{1}$ or $i a_{3}$ implies $2 n+1$ or $n+1+i \equiv 0(m)$ because $a_{1} \equiv-3 n, i a_{3} \equiv-3 i(m)$ and $(3, m)=1$. By $m>3 n$, one sees that this is possible only when $i=2, m=4, n=1$, whence $a_{3}=6 / 2=3$, which implies a contradiction $a_{2}+a_{3} \not \equiv 0(\mathrm{~m})$. Hence $(3.5 .5 .1)$ is proved. Thus one sees $3+3 n-a_{1} \notin \operatorname{ord}\left(C^{\sharp}\right)$ because $3+3 n-a_{1} \leq 1$ by $a_{1}>m>3 n$. Hence $z_{1}=0$. If $z_{2}>0$, then (3.5.3) implies $z=(0, n+1,0)$, because $a_{2}\left(z_{2}-1\right)+a_{3} z_{3}=3 n<m$. Hence we may assume $z_{2}=0$. Then $z_{3} \geq 3$ by (3.5.5.1). Hence by $a_{3}>m / 2 \quad\left(a_{3} \geq 3, a_{3}+3 \geq m\right.$, and $\left.\left(a_{3}, m\right)=1\right)$, one sees that $z_{3} a_{3}>3 m / 2 \geq m+2 \geq 3+3 n$. Thus $z=(0, n+1,0)$ is the only solution. Let us prove (3.5.5). Since $\left(b^{3}-\nu\right)_{3}=b_{3}^{3} \geq 1,\left\|b^{3}\right\|-a_{3} \in \operatorname{ord}\left(C^{\sharp}\right)$. From $\left\|b^{3}\right\|-a_{3} \equiv 3+3 n$ and $3+3 n-m \notin \operatorname{ord}\left(C^{\sharp}\right) \quad(3+3 n-m \leq 2)$, one sees $\left\|b^{3}\right\|-a_{3} \geq 3+3 n$. The rest follows from the above. 
(3.5.6) We claim that $\left\|b^{2}\right\| \geq m+3 n$, and that the equality holds only if $b^{2}=(0, n+1,1)$. By $a_{1}+6 n>m+3 n$ and $a_{3}+3+3 n \geq m+3 n$, one may reduce (3.5.6) to (3.5.4) or (3.5.5) if $b_{1}^{2}$ or $b_{3}^{2}>0$. Hence we assume $b_{1}^{2}=b_{3}^{2}=0$. Then by $b^{2} \neq \nu$, one sees $b_{2}^{2} \geq n+m$ and $\left\|b^{2}\right\| \geq 3 n+3 m$, which proves (3.5.6).

(3.5.7) From (3.5.4)-(3.5.6), one sees

$$
\sum_{i=1}^{3}\left(\left\|b^{i}\right\|-a_{i}\right) \geq m+12 n
$$

and that the equality holds only if $b^{2}=b^{3}=(0, n+1,1)$. This contradicts (3.5.2). Thus (3.1) is proved.

Part II. Imprimitive point $P$. The main result is (3.8).

(3.6) Let $X \supset C \simeq \mathbb{P}^{1}$ be an extremal nbd with an imprimitive point $P$ of index $m$, subindex $\bar{m}(>1)$, and splitting degree $s$. Let $X^{b} \supset C^{b}=C^{b}(1) \cup$ $\ldots \cup C^{b}(s) \ni P^{b}$ be the splitting cover of $X \supset C$ associated to $P(1.12)$. We denote by $X^{b}(i) \supset C^{b}(i) \ni P^{b}(i)$ the extremal nbd obtained from $X^{b} \supset C^{b} \supset P^{b}$ by restriction to a small nbd of $C^{b}(i)$. Let $\left(X^{\sharp}, P^{\sharp}\right) \supset C^{\sharp}=C^{\sharp}(1) \cup \cdots \cup C^{\sharp}(s)$ be the canonical cover of $(X, P)$ and the irreducible components of the pull back $C^{\sharp}$ of $C$ (with reduced structure) such that $C^{\sharp}(i)$ dominates $C^{b}(i)$ for each $i$. We note that $\left(X^{\sharp}, P^{\sharp}\right) \rightarrow\left(X^{b}, P^{b}\right)$ is a $\mu_{m}$-canonical cover. Let $(x)$ and $t$ be normalized $\ell$-coordinates for $X \supset C$ at $P$ and $\phi$ an $\ell$-equation such that wt $x_{1}+$ wt $x_{3} \equiv 0$ and wt $x_{4} \equiv$ wt $\phi(m)$. By (1.14) and (2.9), we have

$$
\operatorname{gr}_{C^{b}}^{0} \omega \simeq\left(\mathscr{O}_{C^{\sharp}} \bar{\omega}\right)^{\mu_{\bar{m}}} \stackrel{\sim}{\longrightarrow} \bigoplus_{i} \operatorname{gr}_{C^{b}(i)}^{0} \omega \simeq \bigoplus_{i}\left(\mathscr{O}_{C^{\sharp}(i)} \bar{\omega}\right)^{\mu_{\bar{m}}}
$$

where $\bar{\omega}$ is a $\mu_{m}$-semi-invariant generator of $\omega_{X^{\sharp}}$ at $P^{\sharp}$.

Hence we have $\boldsymbol{\mu}_{\bar{m}}$-semi-invariants $u_{i} \in \mathscr{O}_{X^{\sharp}, P^{\sharp}}$ for $i \in[1, s]$ such that $\left.u_{i}\right|_{C^{\sharp}(j)}=0$ for $j \in[1, s] \backslash\{i\}$ and $\left(\left.u_{i}\right|_{C^{\sharp}(i)}\right) \bar{\omega}$ is a generator of $\operatorname{gr}_{C^{b}(i)}^{0} \omega$. We also have a $\mu_{m}$-semi-invariant $u \in \mathscr{O}_{X^{\sharp}, P^{\sharp}}$ such that $u \bar{\omega}$ is a generator of $\operatorname{gr}_{C}^{0} \omega$ at $P$. It is easy to see that we may further assume that $u$ is a monomial in $x$ with coefficient 1 . For each $i$, we note that $\bar{m} \cdot w_{P^{b}(i)}(0), \bar{m} \cdot w_{P}(0)$ are nonnegative integers such that $\bar{m} \cdot w_{P^{b}(i)}(0) \equiv \bar{m} \cdot w_{P}(0)(\bmod \bar{m})$ by $(2.10)$ and $\bar{m} \cdot w_{P^{b}(i)}(0), \bar{m} \cdot w_{P}(0)<\bar{m}$ by $(2.3 .3)$. Whence we have

(3.7) Proposition. $w_{P^{b}(i)}(0)=w_{P}(0)$ for all $i$.

Hence we see $C^{\sharp}(i)$-ord $\left(u_{i}\right)=\operatorname{ord}(u)=\bar{m} \cdot w_{P}(0)<\bar{m}$ (2.10). Thus if we consider the induced map

$$
\Phi:\left(\mathscr{O}_{C^{\sharp}, P^{\sharp}} \bar{\omega}\right)^{\mu_{\bar{m}}} \rightarrow \bigoplus_{i} \operatorname{gr}_{C^{b}(i)}^{0} \omega \otimes \mathbb{C}\left(P^{b}(i)\right)=\bigoplus_{i} \mathbb{C}\left(P^{b}\right) u_{i} \bar{\omega},
$$

we see $\Phi(v \bar{\omega})=0$ for $\mu_{m}$-semi-invariants $v$ with $\operatorname{ord}(v)>\bar{m} \cdot w_{P}(0)$. 
(3.8) Proposition. Modulo permutations of $x_{1}, \ldots, x_{4}$ and modulo changes of $\ell$-character $\chi$ (2.5), we have

$$
\text { ow }\left(C^{\sharp}\right)=\mathbb{Z}_{+} \text {ow }\left(x_{1}\right)+\mathbb{Z}_{+} \text {ow }\left(x_{2}\right)
$$

(in particular $\left(C^{\sharp}, P^{\sharp}\right)$ is a planar singularity (2.7)) and one of the following holds :

(3.8.1) wt $\phi \equiv 0 \quad(m)$, wt $x_{1}+$ wt $x_{3} \equiv 0 \quad(m)$, ow $\left(x_{4}\right)=(\bar{m}, 0),(\bar{m}, 0) \in$ $\mathbb{Z}_{++}$ow $\left(x_{1}\right)+\mathbb{Z}_{++}$ow $\left(x_{2}\right)$, and $w_{P}(0) \geq 1 / 2$,

(3.8.2) wt $\phi \equiv 0(m), s=2, \bar{m}$ is an even integer $\geq 4$, and a list of wt and ord for $(x)$ :

$$
\begin{array}{cccccc} 
& x_{1} & x_{2} & x_{3} & x_{4} & \\
\text { wt } & 1 & -1 & 0 & \bar{m}+1 & \bmod m, \\
\text { ord } & 1 & \bar{m}-1 & \bar{m} & \bar{m}+1 &
\end{array}
$$

(3.8.3) $s=\bar{m}=2$, wt $\phi \equiv 2$ (4), and

$$
\begin{array}{cccccc} 
& x_{1} & x_{2} & x_{3} & x_{4} & \\
\text { wt } & 1 & 3 & 3 & 2 & \bmod 4 . \\
\text { ord } & 1 & 1 & 1 & 2 &
\end{array}
$$

Proof. We treat three cases.

(3.8.4) Case wt $\phi \equiv 0(m)$ and ord $x_{2}<\bar{m}$. Then we claim that $u$ is not a power of $x_{2}$. Indeed if $u=x_{2}{ }^{\alpha}$ for some $\alpha$, then $C^{\sharp}$-wt $x_{2}{ }^{\alpha+1} \equiv 0(m)$, whence $\alpha+1 \geq m \geq 2 \bar{m}$ by (wt $\left.x_{2}, m\right)=1$ (1a.5), and ord $u \geq \bar{m}$. This is a contradiction. Thus up to a permutation of $x_{1}$ and $x_{3}$, we may assume $u=x_{1} v$ for some monomial $v$. Then wt $x_{2} u \equiv 0(m)$, whence ord $x_{2} u \equiv 0(\bar{m})$ and ord $x_{2} u<2 \bar{m}$. Hence ord $x_{2} u=\bar{m}$, i.e. $\operatorname{ow}\left(x_{2} u\right)=(\bar{m}, 0)$. By normalizedness, ow $\left(x_{4}\right)=\mathrm{ow}\left(x_{2} u\right)$. We also see wt $x_{2} v \equiv$ wt $x_{2} u-$ wt $x_{1} \equiv$ wt $x_{3} \quad(m)$ and ord $x_{2} v<\operatorname{ord} x_{2} u=\bar{m}$, whence $\operatorname{ow}\left(x_{2} v\right)=\operatorname{ow}\left(x_{3}\right)$ by normalizedness. Thus we have ow $\left(X^{\sharp}\right)=\mathbb{Z}_{+}$ow $\left(x_{1}\right)+\mathbb{Z}_{+}$ow $\left(x_{2}\right)$.

We claim $(\bar{m}, 0) \in \mathbb{Z}_{++}$ow $\left(x_{1}\right)+\mathbb{Z}_{++}$ow $\left(x_{2}\right)$. Indeed if otherwise, we have $(\bar{m}, 0)=\alpha \cdot \operatorname{ow}\left(x_{1}\right) \quad(\alpha \in \mathbb{Z})$ up to permutation of $x_{1}$ and $x_{2}$, whence $\alpha \equiv 0$ $(m)$ by $\left(\right.$ wt $\left.x_{1}, m\right)=1$, which contradicts $\bar{m}=\alpha \cdot \operatorname{ord} x_{1}$ and $m>\bar{m}$. Thus the claim is proved.

We also claim that $w_{P}(0) \geq 1 / 2$. Indeed if otherwise, we have $\bar{m} / 2>$ $\bar{m} \cdot w_{P}(0)=\operatorname{ord} u$. Hence $\operatorname{ord} x_{2}=\bar{m}-\operatorname{ord} u>\bar{m} \cdot w_{P}(0)$ and $\operatorname{ord} x_{3} \geq$ $\bar{m}-\operatorname{ord} x_{1} \geq \bar{m}-\operatorname{ord} u>\bar{m} \cdot w_{P}(0)$, because $x_{1} \mid u$. Thus $\Phi$ factors through

$$
\left(\mathscr{O}_{C^{\sharp}, P^{\sharp}} \bar{\omega} /\left(x_{1}^{\bar{m}}, x_{2}, x_{3}, x_{4}\right)\right)^{\mu_{\bar{m}}} \simeq \mathbb{C}\left(P^{b}\right) x_{1}^{\lambda} \bar{\omega}
$$

for a unique $\lambda \in(0, \bar{m})$ such that $\lambda$ wt $x_{1} \equiv-$ wt $\bar{\omega}(\bar{m})$. This contradicts the surjectivity of $\Phi$ and $s \geq 2$. Thus $w_{P}(0) \geq 1 / 2$ as claimed, and we have case (3.8.1).

(3.8.5) Case ord $x_{2} \geq \bar{m}$ and wt $\phi \equiv 0(m)$. We note first that ord $x_{2}>\bar{m}$ by $\left(\operatorname{ord} x_{2}, \bar{m}\right)=\left(\right.$ wt $\left.x_{2}, \bar{m}\right)=1$ and $\bar{m}>1$. We may assume ord $x_{1} \leq \operatorname{ord} x_{3}$ up 
to a permutation of $x_{1}$ and $x_{3}$, whence $\operatorname{ord}\left(x_{3}{ }^{2}\right) \geq \bar{m}$. Since $x_{1}^{\bar{m}}, x_{2}, x_{1} x_{3}$, $x_{3}{ }^{2}, x_{4}$ have ord $\geq \bar{m}$, we see that $\Phi$ factors through

$$
\left(\mathscr{O}_{C^{\sharp}, P^{\sharp}} \bar{\omega} /\left(x_{1}^{\bar{m}}, x_{1} x_{3}, x_{3}{ }^{2}, x_{2}, x_{4}\right)\right)^{\mu_{\bar{m}}} \simeq \mathbb{C}\left(P^{b}\right) x_{1}^{\lambda} \bar{\omega} \oplus\left(\mathbb{C}\left(P^{b}\right) x_{3} \bar{\omega}\right)^{\mu_{\bar{m}}}
$$

for a unique $\lambda \in(0, \bar{m})$ such that $\lambda \mathrm{wt} x_{1}+\mathrm{wt} \bar{\omega} \equiv 0(\bar{m})$. Hence by surjectivity of $\Phi$, we have $s=2$ and wt $x_{3}+$ wt $\bar{\omega} \equiv 0(\bar{m})$. Since wt $x_{1} \equiv-$ wt $x_{3}(m)$, we see $\lambda \equiv-1(\bar{m})$, whence $\lambda=\bar{m}-1$ and ord $x_{1}=1$ by ord $x_{1}{ }^{\lambda}<\bar{m}$. Thus ord $x_{3}=\bar{m}-1$ by wt $x_{3} \equiv-1$ and ord $x_{3}<\bar{m}$ because $\Phi\left(x_{3}\right) \neq 0$. Hence ow $\left(x_{1}\right)+\mathrm{ow}\left(x_{3}\right)=(\bar{m}, 0)$, whence it is equal to ow $\left(x_{4}\right)$ by the normalizedness as before. Since $\Phi$ factors through $\mathbb{C} x_{1}{ }^{\bar{m}-1} \bar{\omega} \oplus \mathbb{C} x_{3} \bar{\omega}$, we see that $u=x_{1}^{\bar{m}-1}$ or $x_{3}$.

We claim that $u=x_{1}{ }^{\bar{m}-1}$. Indeed if $u=x_{3}$, then wt $x_{2}+$ wt $x_{3} \equiv 0(m)$, and wt $x_{2} \equiv$ wt $x_{1}(m)$ which contradicts normalizedness by ord $x_{2}>1$ and ord $x_{1}=1$. Hence $u=x_{1}^{\bar{m}-1}$.

Thus we have wt $x_{2} \equiv-(\bar{m}-1)$ wt $x_{1} \equiv(\bar{m}+1)$ wt $x_{1}(m)$. Since ord $x_{2}>\bar{m}$ and ord $x_{1}{ }^{\bar{m}+1}=\bar{m}+1$, we have ow $\left(x_{2}\right)=(\bar{m}+1)$ ow $\left(x_{1}\right)$ by normalizedness, whence ow $\left(C^{\sharp}\right)=\mathbb{Z}_{+}$ow $\left(x_{1}\right)+\mathbb{Z}_{+}$ow $\left(x_{3}\right)$. By $\left(\right.$ wt $\left.x_{2}, m\right)=$ $\left((\bar{m}+1)\right.$ wt $\left.x_{1}, m\right)=1$, we see $\bar{m} \equiv 0 \quad(2)$. By changing $\ell$-character $\chi$ with $(1+\bar{m}) \chi$ if necessary, we may assume ow $\left(x_{1}\right)=(1,1)$, ow $\left(x_{2}\right)=(\bar{m}+1, \bar{m}+1)$, $\operatorname{ow}\left(x_{4}\right)=(\bar{m}, 0)$. Since $\mathrm{ow}\left(x_{3}\right) \notin \mathbb{Z}_{+}$ow $\left(x_{1}\right)$ (otherwise $\left(C^{\sharp}, P^{\sharp}\right)$ is smooth (2.7)), we have ow $\left(x_{3}\right)=(\bar{m}-1,-1)$. We note that if $\bar{m}=2$, then permuting $x_{2}$ and $x_{3}$, we have case (3.8.1). Hence we may assume $\bar{m} \geq 4$, and by permuting $x_{i}$ 's, we have case (3.8.2).

(3.8.6) Case $m=4, \bar{m}=s=2$, wt $\phi \equiv 2$ (4). Then we have wt $x \equiv$ $(1,3,3,2) \bmod 4$ up to permutation of $x_{1}, \ldots, x_{4}$ and a choice of $\ell$-character $\chi$ (1a.5). Then we have wt $u \equiv 1$ (4) and ord $u=1$. Hence $u=x_{1}$ and ord $x_{1}=1$, whence ow $\left(x_{4}\right)=\operatorname{ow}\left(x_{1}{ }^{2}\right)$. Since $\bar{m} \cdot w_{P}(0)=1, \Phi$ factors through $\mathbb{C} x_{1} \bar{\omega}+\mathbb{C} x_{2} \bar{\omega}+\mathbb{C} x_{3} \bar{\omega}$. Thus ord $x_{2}=1$ or ord $x_{3}=1$ by surjectivity of $\Phi$, and ord $x_{2}=$ ord $x_{3}=1$ by normalizedness. Hence ow $\left(C^{\sharp}\right)=\mathbb{Z}_{+}$ow $\left(x_{1}\right)+$ $\mathbb{Z}_{+}$ow $\left(x_{2}\right)$, and we have case (3.8.3).

\section{Classification of $X \supset C$ at $P$ into cases}

After classifying $X \supset C \ni P$ into several cases, we will study the deformation processes using results in $\S \S 1 \mathrm{a}$ and $1 \mathrm{~b}$. These processes allow us to treat fewer cases with only ordinary singular points $(4.5)$.

(4.1) Let $X \supset C \simeq \mathbb{P}^{1}$ be an extremal nbd, and $P \in C$ a singular point of $X$ of index $m \geq 1$, subindex $\bar{m}$, splitting degree $s$. We note that $m=\bar{m} \cdot s$ and that $s=1$ iff $P$ is primitive. We consider the germs $(X, P) \supset(C, P)$, and use the notation in $\S 2$ like $\mu_{m}$-cover $\left(X^{\sharp}, P^{\sharp}\right) \supset\left(C^{\sharp}, P^{\sharp}\right)$ of $X \supset C$, etc. One has $\operatorname{emb}^{\sharp} C^{\sharp} \leq 2$ by (3.1) and (3.8). So, let $(x)=\left(x_{1}, \ldots, x_{4}\right)$ and $t$ be 
normalized $\ell$-coordinates (2.6) and $\phi$ an $\ell$-equation of $X \supset C \ni P$ (1a.5) such that $x_{1}$ and $x_{2}$ form coordinates for $\left(C^{\sharp}, P^{\sharp}\right)$. Then $\left(a_{1}, w_{1}\right)$ and $\left(a_{2}, w_{2}\right)$ generate ow $\left(C^{\sharp}\right)$, where $\mathrm{ow}_{i}=\left(a_{i}, w_{i}\right)=\mathrm{ow}\left(x_{i}\right) \quad(i \in[1,4])$.

We note that $U\left(s \cdot a_{1} a_{2}\right)=U\left(s a_{1} \cdot \mathrm{ow}_{2}\right)=U\left(s a_{2} \cdot \mathrm{ow}_{1}\right)$ (cf. (2.8)) depends only on ow $\left(C^{\sharp}\right)$ and $m$. Indeed if $\operatorname{ow}\left(C^{\sharp}\right) \simeq \mathbb{Z}_{+}$, then $a_{1}$ or $a_{2}=1$ and $a_{1}, a_{2} \leq m$, and $U\left(a_{1} a_{2}\right)=1$; if ow $\left(C^{\sharp}\right) \not \mathbb{Z}_{+}$, then

$$
\text { ow } \left._{1}, \text { ow }_{2}\right\}=\left(\text { ow }\left(C^{\sharp}\right)-\{0\}\right) \backslash\left(\left(\text { ow }\left(C^{\sharp}\right)-\{0\}\right)+\left(\operatorname{ow}\left(C^{\sharp}\right)-\{0\}\right)\right)
$$

and $U\left(s \cdot a_{1} a_{2}\right)$ depends only on $\operatorname{ow}\left(C^{\sharp}\right)$ and $m$.

(4.1.1) Definition. We call $U\left(s \cdot a_{1} a_{2}\right)$ the size of $X \supset C \ni P$ (or simply size of $P$ ) and denote it by $\operatorname{siz}_{P}$ (cf. (4.10)).

(4.2) Proposition-Definition. Modulo permutations of $x_{i}$ 's (and changes of $\ell$-characters in case of imprimitive points) which preserve the condition

$$
\operatorname{ow}\left(C^{\sharp}\right)=\mathbb{Z}_{+} \text {ow }_{1}+\mathbb{Z}_{+} \text {ow }_{2},
$$

exactly one of the following cases holds. This division is independent of the choice of normalized $\ell$-coordinates $(x)$ and $t$.

( Primitive point $P$ )

Case (I) $m>1, C^{\sharp}$-wt $\phi \equiv 0(m)$,

(IA) $a_{1}+a_{3} \equiv 0(m), a_{4}=m, m \in \mathbb{Z}_{++} a_{1}+\mathbb{Z}_{++} a_{2}$, where we may still permute $x_{1}$ and $x_{3}$ if $a_{2}=1$,

(IB) $a_{1}+a_{3} \equiv 0(m), a_{2}=m, 2 \leq a_{1}$,

(IC) $a_{1}+a_{2}=a_{3}=m, a_{4} \not \equiv a_{1}, a_{2}, 2 \leq a_{1}<a_{2}$.

Case (II) $m=4, C^{\sharp}$-wt $\phi \equiv 2$ (4), $\phi \in(x)^{2}$,

(IIA) $\left(a_{1}, \ldots, a_{4}\right)=(1,1,3,2)$,

(IIB) $\left(a_{1}, \ldots, a_{4}\right)=(3,2,5,5)$, and

Case (III) $m=1$. In this case, $X^{\sharp}=X$ and $C^{\sharp}=C$.

(Imprimitive point $P$ )

Case $\left(\mathrm{I}^{\vee}\right) \bar{m}, s>1, C^{\sharp}$-wt $\phi \equiv 0(m)$, $1 / 2$,

$\left(\mathrm{IA}^{\vee}\right) w_{1}+w_{3} \equiv 0(m)$, ow $\left(x_{4}\right)=(\bar{m}, 0) \in \mathbb{Z}_{++} \mathrm{ow}_{1}+\mathbb{Z}_{++}$ow $\mathrm{w}_{2}$, and $w_{P}(0) \geq$

$\left(\mathrm{IC}^{\vee}\right) s=2, \bar{m}$ is an even integer $\geq 4$, and

$$
\begin{array}{cccccc} 
& x_{1} & x_{2} & x_{3} & x_{4} & \\
\text { wt } & 1 & -1 & 0 & \bar{m}+1 & \bmod m, \\
\text { ord } & 1 & \bar{m}-1 & \bar{m} & \bar{m}+1 &
\end{array}
$$

Case (II $\left.{ }^{\vee}\right) s=\bar{m}=2$, wt $\phi \equiv 2$ (4), $\phi \in(x)^{2}$, and

$$
\begin{array}{cccccc} 
& x_{1} & x_{2} & x_{3} & x_{4} & \\
\text { wt } & 1 & 3 & 3 & 2 & \bmod 4 . \\
\text { ord } & 1 & 1 & 1 & 2 &
\end{array}
$$


Depending on the case occurring, we say that $X \supset C \ni P($ or $X \supset C$ at $P$, or simply $P$ (if there is no danger of confusion)) is of type (IA), .., (III), $\left(\mathrm{IA}^{\vee}\right), \ldots,\left(\mathrm{II}^{\vee}\right)$.

Proof. The case of imprimitive points was treated in (3.8). Now assume that $P$ is primitive. First we show that one of cases (IA), .., (III) occurs.

In case (I), one has $a_{i}+a_{j} \equiv 0(m), a_{k}=m$ for some distinct $i, j, k \in$ [1,4] by normalizedness of $(x)$ and $t$ and (1a.6). If $\operatorname{ord}\left(C^{\sharp}\right)=\mathbb{Z}_{+}$, then $\left(a_{\sigma(1)}, \ldots, a_{\sigma(4)}\right)=(1, b, m-1, m)$ or $(b, 1, m-b, m)$ for some $b \in[1, m-1]$ and $\sigma \in \mathfrak{S}_{4}$, where $\mathbb{Z}_{+} a_{\sigma(1)}+\mathbb{Z}_{+} a_{\sigma(2)}=\mathbb{Z}_{+}$is maintained. Thus we may assume $a_{1}, a_{2} \geq 2$, and treat three cases modulo (12) and (34) $\in \mathfrak{S}_{4}$.

(4.2.1) Case $a_{1}+a_{3} \equiv 0, a_{2}$ or $a_{4}=m$. If $a_{4}=m$, then $m \notin \mathbb{Z}_{+} a_{1} \cup \mathbb{Z}_{+} a_{2}$ by $\left(m, a_{1} a_{2}\right)=1$, and we have (IA). Otherwise, we have (IB).

(4.2.2) Case $a_{1}+a_{2} \equiv 0, a_{3}=m$, and $2 \leq a_{1} \leq a_{2}$. If $a_{4} \equiv a_{1}$ or $a_{2}$, this belongs to (4.2.1) modulo (34) $\in \mathfrak{S}_{4}$. Otherwise, we have (IC), because $a_{1} \neq a_{2}$ by $\left(m, a_{1}\right)=1$ and $m \in\left(a_{1}, a_{2}\right)$.

(4.2.3) Case $a_{3}+a_{4} \equiv 0, a_{2}=m$. Since $a_{2}=m$ and $a_{1} \not \equiv 0(m)$, we have $R\left(a_{1}\right) \in \mathbb{Z}_{++} a_{1}$. By $(2.3 .3)$ and (2.10), one has $R\left(a_{1}\right)=m \cdot w_{P}(0)<m$, whence $a_{1}<m$. Thus $a_{1}+R\left(a_{1}\right)=m$, because $a_{1}+R\left(a_{1}\right) \quad(<2 m)$ belongs to $\mathbb{Z}_{+} m$. Hence $m \in \mathbb{Z}_{+} a_{1}$, which contradicts $\left(m, a_{1}\right)=1$. Thus (4.2.3) does not occur, and case (I) is finished.

(4.2.4) In case (II), one has $a_{1}, \ldots, a_{4} \not \equiv 0$ (4) (1a.6) and $4 \in\left(\operatorname{ord}\left(C^{\sharp}\right)-\{0\}\right)+$ $\left(\operatorname{ord}\left(C^{\sharp}\right)-\{0\}\right)$, whence $2 \in \operatorname{ord}\left(C^{\sharp}\right)$. Since $4 w_{P}(0) \quad(\in \mathbb{N})$ is prime to 4 and $4 w_{P}(0)<4$ by $(2.3 .3)$ and $(2.10)$, one has $3 \in \operatorname{ord}\left(C^{\sharp}\right)$. Thus $\operatorname{ord}\left(C^{\sharp}\right)=\mathbb{Z}_{+}$ or $\mathbb{Z}_{+}-\{1\}$. Hence by $C^{\sharp}$-wt $(x) \equiv(1,2,3,1),(1,2,3,3) \bmod 4$ and modulo permutation, one has $C^{\sharp}$-ord $(x)=(1,2,3,1),(3,2,5,5),(1,2,3,3)$, or $(3,2,5,3)$ modulo permutation. The last case is impossible by (2.3.3), because one has $w_{P}(0)=5 / 4>1$ by $(2.10)$. In the third case, one sees that $x_{1}{ }^{2}$ must appear in the power series expansion of $\phi$ by (1a.6) and wt $x_{1} \not \equiv$ wt $x_{3}$, wt $x_{4}$ (4). Thus by $\phi \in\left(x_{2}-x_{1}{ }^{2}, x_{3}-x_{1}{ }^{3}, x_{4}-x_{1}{ }^{3}\right)$, one has $\partial \phi / \partial x_{2}(0) \neq 0$, which contradicts the assumption $\phi \in(x)^{2}$ of case (II). From the first two cases, one has (IIA) or (IIB).

(4.2.5) We will show that there are no overlaps in cases and the division does not depend on choice of $(x)$ and $t$. By normalizedness, $a_{1}, \ldots, a_{4}$ (modulo permutation) is independent of choice of $(x)$ and $t$ (2.6). In case (II), we have (IIA) iff ord $\left(C^{\sharp}\right)=\mathbb{Z}_{+}$. We only need to consider case (I). If $\operatorname{ord}\left(C^{\sharp}\right)=$ $\mathbb{Z}_{+}$, then we have (IA), and we are done. Assume that $\operatorname{ord}\left(C^{\sharp}\right) \neq \mathbb{Z}_{+}$, where $\operatorname{ord}\left(C^{\sharp}\right)$ needs exactly two generators $a_{1}$ and $a_{2}$. One has (IB) iff $m$ is one of the generators. In case (IC), there is exactly one solution $(i, j)=(1,2)$ for $a_{i}+a_{j} \equiv 0(m) \quad(1 \leq i<j \leq 4)$, and it is given by the generators $a_{1}$ and $a_{2}$. Therefore (IA) and (IC) do not occur at the same time. 
(4.3) Let $f_{3}$ and $f_{4}$ be monomials in $x_{1}$ and $x_{2}$ such that

$$
x_{i}=f_{i}\left(x_{1}, x_{2}\right) \text { on } C^{\sharp}(i=3,4) \text {. }
$$

Then

$$
F_{2}=x_{1}^{s a_{2}}-x_{2}^{s a_{1}}, \quad F_{3}=x_{3}-f_{3}, \quad F_{4}=x_{4}-f_{4}
$$

generate the defining ideal $J^{\sharp}$ of $\left(C^{\sharp}, P^{\sharp}\right)$ in $\left(\mathbb{C}^{4}, 0\right)$. Then

$$
\phi=g_{2} \cdot F_{2}+g_{3} \cdot F_{3}+g_{4} \cdot F_{4}
$$

for some semi-invariant $g_{i}$ 's $\in \mathbb{C}\{x\}$ with wt $g_{i} \equiv$ wt $\phi-$ wt $F_{i}$.

(4.4) Lemma. Under the notation of (4.2), one has

(i) $\left(X^{\sharp}, P^{\sharp}\right)$ is smooth and $J^{\sharp}=\left(F_{2}, F_{4}, \phi\right)$ if $P$ is of type (IC) or $\left(\mathrm{IC}^{\vee}\right)$, and

(ii) $J^{\sharp}=\left(F_{3}, F_{4}, \phi\right)$ if $P$ is of type (IIB) or ( $\left.\mathrm{II}^{\vee}\right)$.

Proof. (i) One sees that $f_{3}$ must be $x_{1} x_{2}$, and

$$
\phi=g_{2} \cdot\left(x_{1}^{s a_{2}}-x_{2}^{s a_{1}}\right)+g_{3} \cdot\left(x_{3}-x_{1} x_{2}\right)+g_{4} \cdot\left(x_{4}-f_{4}\right) \text {. }
$$

Since $P$ is of type (IC) or ( IC $\left.{ }^{\vee}\right)$, one sees that $m=s\left(a_{1}+a_{2}\right)>4$, and $g_{2} \in(x)$, and that $g_{4}, f_{4} \in(x)^{2}$ by wt $x_{4} \not \equiv 0, \pm \mathrm{wt} x_{1}, \pm \mathrm{wt} x_{2}(m)$. Since $m \geq 5$, one sees that $x_{1} x_{2}$ or $x_{3}$ must appear in the power series expansion of $\phi$ (1a.5). By $s a_{1}, s a_{2} \geq 2$, this is only possible if $g_{3}$ is a unit. Thus $\left(X^{\sharp}, P^{\sharp}\right)$ is smooth and $\left(F_{2}, F_{3}, F_{4}\right)=\left(F_{2}, F_{3}, \phi\right)$.

(ii) By (1a.6), $x_{1}{ }^{2}$ must appear in the power series expansion of $\phi$, since wt $x_{1} \not \equiv$ wt $x_{2}$, wt $x_{3}$, wt $x_{4}$ (4). One sees that $f_{3}$ and $f_{4}$ must be $x_{1} x_{2}$ if $P$ is of type (IIB) (resp. $f_{3}=x_{2}$ and $f_{4} \in(x)^{2}$ if $P$ is of type $\left(\mathrm{II}^{\vee}\right)$ ). Thus similarly in

$$
\phi=g_{2} \cdot\left(x_{1}{ }^{2}-x_{2}^{s a_{1}}\right)+g_{3} \cdot\left(x_{3}-f_{3}\right)+g_{4} \cdot\left(x_{4}-f_{4}\right),
$$

we see that $g_{4} \in(x)$ by $\phi \in(x)^{2}$, and hence that $g_{2}$ is a unit, whence $\left(F_{2}, F_{3}, F_{4}\right)=\left(F_{3}, F_{4}, \phi\right)$.

(4.5) Definition. Let $X \supset C \simeq \mathbb{P}^{1}$ be an extremal nbd, and $P \in C$ be a singular point of $X$.

(i) We say $X \supset C$ is ordinary at $P$ (or $P$ is an ordinary point) if $(X, P)$ is an ordinary double point or a cyclic quotient singularity (i.e. the canonical cover $\left(X^{\sharp}, P^{\sharp}\right)$ is smooth), and

(ii) We say that $P$ is almost ordinary iff $P$ is of type (IB) and $\left(C^{\sharp}, P^{\sharp}\right)$ is a complete intersection in $\left(X^{\sharp}, P^{\sharp}\right)$.

(4.5.1) Remark. (i) $P$ is an ordinary point iff either

(a) $P$ is of type (IA) or $\left(\mathrm{IA}^{\vee}\right)$, and $(X, P)$ is a cyclic quotient singularity $\left(\Leftrightarrow J^{\sharp}=\left(\phi, F_{2}, F_{3}\right) \Leftrightarrow\left(C^{\sharp}, P^{\sharp}\right)\right.$ is a complete intersection in $\left.\left(X^{\sharp}, P^{\sharp}\right)\right)$, 
(b) $P$ is of type (IC) or $\left(\mathrm{IC}^{\vee}\right)$, or

(c) $P$ is of type (III), and $(X, P)$ is an ordinary double point (i.e. $(X, P) \supset$ $C$ is given as $(\{x y=z u\}, 0) \supset x$-axis for suitable coordinates $(x, y, z, u))$,

(ii) a type (IB) point $P$ is almost ordinary iff $J^{\sharp}=\left(\phi, F_{3}, F_{4}\right)$.

Proof. We treat (i) first. If $P$ is of type (IA) or (IA $\left.{ }^{\vee}\right)$, then $F_{4}=x_{4}-f_{4}\left(x_{1}, x_{2}\right)$ and wt $\phi \not \equiv$ wt $F_{2}$, wt $F_{3}(m)$. Therefore the equivalence in case (a) is clear. If $P$ is of type (IB), then $\phi \in(x)^{2}$ by $\phi \in\left(F_{2}, F_{3}, F_{4}\right), F_{2} \in(x)^{2}$ and wt $\phi \not \equiv$ wt $F_{3}$, wt $F_{4}(m)$. Thus $P$ is not ordinary. (4.4(i)) treats points of type (IC), (IC ${ }^{\vee}$ ). Points of type (IIA), (IIB), (II $\left.{ }^{\vee}\right)$ are never ordinary by $\phi \in(x)^{2}$. (ii) follows from wt $\phi \not \equiv$ wt $F_{3}$, wt $F_{4}(m)$.

Points of type (IA) or $\left(\mathrm{IA}^{\vee}\right)$ require delicate analysis. For these, we state the following technical lemma to be used in $\S 9$.

(4.5.2) Lemma. If $P$ is an ordinary point of type (IA) or (IA $\left.{ }^{\vee}\right)$ such that $\operatorname{siz}_{P}=1$ and $\left(C^{\sharp}, P^{\sharp}\right)$ is singular, then $\left\{F_{2}, F_{3}\right\}$ is a free basis for $\operatorname{gr}_{C^{\sharp}}^{1} \mathscr{O}$ at $P^{\sharp}$ such that $\mathrm{ow}^{*}\left(F_{2}\right)=(\bar{m}, 0)-s a_{1} \cdot \mathrm{ow}_{2}, \mathrm{ow}^{*}\left(F_{3}\right)=\mathrm{ow}_{1} \quad(2.8), R\left(\right.$ wt $\left.F_{2}\right)>$ $R\left(\right.$ wt $\left.F_{3}\right)$, and

$$
\operatorname{ow}^{*}\left(F_{2}\right)-\mathrm{ow}^{*}\left(F_{3}\right),(\bar{m}, 0)+\mathrm{ow}^{*}\left(F_{3}\right)-\mathrm{ow}^{*}\left(F_{2}\right) \in \mathrm{ow}\left(C^{\sharp}\right) .
$$

Proof. By wt $F_{2} \equiv s a_{1} a_{2}$ and wt $F_{3} \equiv-$ wt $x_{1} \quad(m)$, we see $\mathrm{ow}^{*}\left(F_{2}\right)=(\bar{m}, 0)-$ $s a_{1} \cdot \mathrm{ow}_{2}$ and $\mathrm{ow}^{*}\left(F_{3}\right)=\mathrm{ow}_{1}$ by $\mathrm{siz}_{P}=U\left(s a_{1} \cdot \mathrm{ow}_{2}\right)=1$ and $a_{1}<\bar{m}$. We have ow ${ }^{*}\left(F_{2}\right) \in \mathbb{Z}_{+}$ow $_{1}+\mathbb{Z}_{+}$ow $_{2}(2.8)$, and claim further that ow $^{*}\left(F_{2}\right) \in$ $\mathbb{Z}_{++} \mathrm{ow}_{1}+\mathbb{Z}_{++} \mathrm{ow}_{2}$. Indeed if otherwise, we have $(\bar{m}, 0)=\alpha \cdot \mathrm{ow}_{i}$ for some $\alpha \in \mathbb{Z}_{+}, i \in[1,2]$, whence $\alpha \equiv 0(m)$ by $\left(\right.$ wt $\left.x_{1}, m\right)=\left(\right.$ wt $\left.x_{2}, m\right)=1$ and thus $\bar{m}=m$ and $a_{i}=1$ by $\bar{m}=\alpha a_{i}$, which implies that $P$ is a type (IA) point and $\left(C^{\sharp}, P^{\sharp}\right)$ is smooth. This contradicts our original assumption. Thus we have ow $\left(F_{2}\right) \in \mathbb{Z}_{++} \mathrm{ow}_{1}+\mathbb{Z}_{++} \mathrm{ow}_{2}$, hence $R\left(\mathrm{wt} F_{2}\right)-R\left(\mathrm{wt} F_{3}\right)>0$, and $\operatorname{ow}^{*}\left(F_{2}\right)-\mathrm{ow}^{*}\left(F_{3}\right) \in \mathrm{ow}\left(C^{\sharp}\right)$. Then it is obvious that $(\bar{m}, 0)+\mathrm{ow}^{*}\left(F_{3}\right)-$ $\mathrm{ow}^{*}\left(F_{2}\right)=s a_{1} \cdot \mathrm{ow}_{2}+\mathrm{ow}_{1} \in \mathrm{ow}\left(C^{\sharp}\right)$.

(4.6) Let $X \supset C \simeq \mathbb{P}^{1}$ be an extremal nbd with a singular point $P \in C$ of $X$ of index $m$, and let $P_{1}, \ldots, P_{r}$ be all the other singular points of $X$ on $C$. Let $\left(X^{\sharp}, P^{\sharp}\right) \supset\left(C^{\sharp}, P^{\sharp}\right)$ be the $\mu_{m}$-canonical cover of $(X, P)$ and the preimage of $C$. Let $x=\left(x_{1}, \ldots, x_{4}\right)$ and $t$ be normalized $\ell$-coordinates giving embedding $\left(X^{\sharp}, P^{\sharp}\right) \subset\left(\mathbb{C}^{4}, 0\right)$ such that $a_{i}=C^{\sharp}-\operatorname{ord}\left(x_{i}\right) \quad(i \in[1,4])$ are as in (4.2). Let $\phi \in \mathbb{C}\{x\}$ be an $\ell$-equation of $(X, P)$, and $J^{\sharp} \subset \mathbb{C}\{x\}$ the ideal defining $C^{\sharp}$ in $\left(\mathbb{C}^{4}, 0\right)$, and let $F_{2}, F_{3}, F_{4}$ be as in (4.3). The following is the deformation process.

Let $\theta=x_{2}$ if $P$ is an almost ordinary type (IB) point or a type (IIB) point, and $\theta=0$ otherwise. Let $C_{\lambda}^{\sharp} \subset\left(\mathbb{C}^{4}, 0\right)$ be defined by $F_{2}+\lambda \theta=F_{3}=F_{4}=0$ 
for $\lambda \in \mathbb{C}$. Then we follow step (L) in $\S 1$ b. We will choose semi-invariant $\psi \in(x) \mathbb{C}\{x\}$ with wt $\psi \equiv$ wt $\phi(m)$ such that $\phi_{\lambda}=\phi+\lambda \psi$ induces 0 on $C_{\lambda}^{\sharp}$ and such that $X_{\lambda, \varepsilon}(\ni P=0)$ has only terminal singularities if $|\lambda| \ll \varepsilon \ll 1$, where we introduce $B_{\varepsilon}=\left\{(x)|| x_{i} \mid<\varepsilon(\forall i)\right\}, X_{\lambda, \varepsilon}^{\sharp}=X_{\lambda}^{\sharp} \cap B_{\varepsilon}, X_{\lambda, \varepsilon}=X_{\lambda, \varepsilon}^{\sharp} / \mu_{m}$, $C_{\lambda, \varepsilon}^{\sharp}=C_{\lambda}^{\sharp} \cap B_{\varepsilon}, C_{\lambda, \varepsilon}=C_{\lambda, \varepsilon}^{\sharp} / \mu_{m}$ (this part will be studied more closely case by case later in the proof). Then step (LG) embeds the deformation $X_{\lambda, \varepsilon} \supset C_{\lambda, \varepsilon} \ni$ $P$ to a deformation $X_{\lambda} \supset C_{\lambda} \simeq \mathbb{P}^{1}$ which is trivial outside $X_{\lambda, \varepsilon}$ for $|\lambda| \ll \varepsilon \ll 1$ (1b.7). Thus by (G) in $\S 1 \mathrm{~b}$, one has nearby extremal nbds $X_{\lambda}^{\circ} \supset C_{\lambda} \simeq \mathbb{P}^{1}$ such that $X_{\lambda}^{\circ} \subset X_{\lambda}$ for $|\lambda| \ll 1$.

(4.7) Proposition-Definition. For suitable choice of $\psi$, each nearby extremal nbd $X_{\lambda}^{\circ} \supset C_{\lambda} \simeq \mathbb{P}^{1} \quad(|\lambda| \ll \varepsilon \ll 1)$ contains $P, P_{1}, \ldots, P_{r}$ in the natural way mentioned above so that $\left(X_{\lambda}^{\circ}, P_{i}\right) \supset\left(C_{\lambda}, P_{i}\right)$ is naturally isomorphic to $\left(X, P_{i}\right) \supset$ $\left(C, P_{i}\right)$ for $i \in[1, r]$ and $X_{\lambda, \varepsilon} \supset C_{\lambda, \varepsilon}$ contains all the singularities $\left(\in C_{\lambda}\right)$ of $X_{\lambda} \supset C_{\lambda}$ other than $P_{1}, \ldots, P_{r}$. If $P$ is a primitive (resp. an imprimitive) point, then $X_{\lambda, \varepsilon} \supset C_{\lambda, \varepsilon}$ is locally primitive (resp. $P$ is an imprimitive point of $X_{\lambda, \varepsilon} \supset C_{\lambda, \varepsilon}$ with the same subindex and splitting degree as $\left.X \supset C \ni P\right)$. Depending on the type of $X \supset C \ni P$, one has

Type (IA) (resp. (IB) but not almost ordinary, (IA $\left.{ }^{\vee}\right)$ ). $P$ is the only singularity of $X_{\lambda, \varepsilon}$ on $C_{\lambda, \varepsilon}$, and it is an ordinary type (IA) (resp. an almost ordinary type (IB), an ordinary type $\left(\mathrm{IA}^{\vee}\right)$ ) point, and one can use the same $(x), t, \mathrm{ow}_{i}$ 's, $F_{i}$ 's, except for the $\ell$-equation which is $\phi_{\lambda}=0$, whence size, $w(0)$ remain the same.

Type (IIA) (resp. ( $\left.\left.\mathrm{II}^{\vee}\right)\right) . P$ is the only singularity of $X_{\lambda, \varepsilon}$ on $C_{\lambda, \varepsilon}$, and it is an ordinary type (IA) (resp. $\left(\mathrm{IA}^{\vee}\right)$ ) point, with the same index, size, and $w(0)$ as $X \supset C$ at $P$.

Type (IB) and almost ordinary. $X_{\lambda, \varepsilon}$ has exactly $a_{1}$ singular points (including $P$ ) on $C_{\lambda, \varepsilon}$, which are ordinary points of type (IA) with index $m$, and the invariant $w(0)$ for $X_{\lambda, \varepsilon} \supset C_{\lambda, \varepsilon}$ takes the same value for these $a_{1}$ points.

Type (IIB). $X_{\lambda, \varepsilon}$ has exactly two singular points $P$ and $Q$ on $C_{\lambda, \varepsilon}$, which are ordinary points of type (IA), with indices 4 and 2 , respectively, and

Type (III). $X_{\lambda, \varepsilon}$ has exactly $n$ singular points, and they are on $C_{\lambda, \varepsilon}$ and of type (III), where $n$ is the invariant $i_{P}(1)$ for $X \supset C$ at $P$. If $n=1$, then the singular point on $C_{\lambda, \varepsilon}$ is ordinary.

We call all these processes ( or $X_{\lambda}^{\circ} \supset C_{\lambda}$, by abuse of language) L-deformations at $P$. The L-deformation at a type (III) point $P$ is also called a separating $L$-deformation.

In case of type (III), one can also make $X_{\lambda, \varepsilon}$ smooth when $0<|\lambda| \ll \varepsilon \ll 1$ by choosing some other suitable $\psi$. We call this process an $L$-smoothing at $P$. (L-deformation will mean separating L-deformation for type (III) points unless otherwise mentioned. ) 
Proof. First we note that, if $P$ is an almost ordinary point of type (IB) or a point of type (IIB), $F_{2}+\lambda x_{2}$ is semi-invariant and $C_{\lambda}^{\sharp}$ is smooth in a small enough nbd of $\left(\mathbb{C}^{4}, 0\right)$ when $0<|\lambda| \ll 1(1 \mathrm{~b} .4)$. Thus the assertion on local primitiveness (or imprimitiveness) follows from (1b.3.1) if other parts are done. We treat several cases depending on the type of $P$.

(4.7.1) (IA), (IIA), (IA $\left.{ }^{\vee}\right),\left(\mathrm{II}^{\vee}\right)$ (resp. (IB) but not almost ordinary). We choose semi-invariant general $\psi \in F_{4}+\mathbb{C}\{x\} F_{2}+\mathbb{C}\{x\} F_{3}$ (resp. $F_{2}+$ $\left.\left(\mathbb{C}^{*} x_{1}+(x)^{2} \mathbb{C}\{x\}\right) F_{3}+\mathbb{C}\{x\} F_{4}\right)$ with wt $\psi \equiv$ wt $\phi(m)$. We note that $\{\phi=$ $\psi=0\}$ is a normal surface by Bertini's Theorem because $\left((x)^{2} J^{\sharp}\right)^{\boldsymbol{\mu}_{m}} \cdot \mathbb{C}\{x\}$ defines a closed subscheme $\subset\left(\mathbb{C}^{4}, 0\right)$ which is equal to $C^{\sharp}$ except at a finite number of points. Thus $(1 \mathrm{~b} .3(\mathrm{i}))$ is satisfied, and $\left(1 \mathrm{~b} .5\left(\mathrm{ii}^{\prime}\right)\right)$ (resp. $\left(1 \mathrm{~b} .5\left(\mathrm{ii}^{\prime \prime}\right)\right)$ is satisfied by the choice of $\psi$. Thus $X_{\lambda, \varepsilon}$ has only terminal singularities and these come from points in $X_{\lambda, \varepsilon}^{\sharp} \cap x_{a}$-axis for $a=4$ (resp. $a=2$ ) (1b.3). Since $C^{\sharp} \cap x_{a}$-axis $=\{0\}, P$ is the only singular point of $X_{\lambda, \varepsilon}$ lying on $C$. We note $J^{\sharp}=\left(\phi_{\lambda}, F_{2}, F_{3}\right)$ (resp. $\left.J^{\sharp}=\left(\phi_{\lambda}, F_{3}, F_{4}\right)\right)$. The assertions on size, $w(0)$ follow from definition and (2.10).

(4.7.2) Almost ordinary and (IB), or (IIB). We write $\phi$ as $\phi=g_{2} F_{2}+g_{3} F_{3}+$ $g_{4} F_{4}$, where $g_{2}, g_{3}, g_{4}$ are semi-invariants such that wt $g_{i} \equiv$ wt $\phi-$ wt $F_{i}$. We note that $g_{2}$ is a unit by the hypothesis or (4.4(ii)). We choose semiinvariant general $\psi \in g_{2} x_{2}+\mathbb{C}\{x\} F_{3}+\mathbb{C}\{x\} F_{4}$ with wt $\psi \equiv$ wt $\phi(m)$. Thus $\phi_{\lambda} \in g_{2}\left(F_{2}+\lambda x_{2}\right)+\mathbb{C}\{x\} F_{3}+\mathbb{C}\{x\} F_{4}$, and $\phi_{\lambda} \equiv 0$ on $C_{\lambda}^{\sharp}$. Then $\psi \notin(x)^{2}$ since $g_{2}$ is a unit. One can check (1b.3(i)) similarly to the previous case. By (1b.3) and $\psi \notin(x)^{2}, X_{\lambda, \varepsilon}$ has only terminal cyclic quotient singularities and they are images of $X_{\lambda, \varepsilon}^{\sharp} \cap x_{2}$-axis. If $P$ is of type (IB), $C_{\lambda}^{\sharp} \cap x_{2}$-axis $=\{(0, \xi, 0,0) \mid$ $\left.\xi^{a_{1}}=\lambda \xi\right\}$ and $x_{1}, x_{3}, x_{4}$ form coordinates for $X_{\lambda}^{\sharp}$ at all these $a_{1}$ points by $\partial \phi_{\lambda} / \partial x_{2} \neq 0$. Since $x_{1}$ is a uniformizant for $C_{\lambda}^{\sharp}$ at all these points, $\boldsymbol{\mu}_{m}$ is the stabilizer of each point, and $\mathrm{wt}(x)$ are the same at these points, one sees that they have index $m$ and the same $w(0)$ by (2.10). If $P$ is of type (IIB), one has $C_{\lambda}^{\sharp} \cap x_{2}$-axis $=(0)$ or $(0, \pm \sqrt{\lambda}, 0,0)$. Stabilizer of $(0)$ is $\boldsymbol{\mu}_{4}$, and stabilizers of the conjugate $(0, \pm \sqrt{\lambda}, 0,0)$ are $\mu_{2}$. So we have two points with indices 4 and 2 .

(4.7.3) (III). In this case, we choose coordinates $\left(z_{1}, \ldots, z_{4}\right)$ so that $C$ is $z_{1}$-axis and $\phi \equiv z_{1}{ }^{n} z_{2}\left(z_{2}, z_{3}, z_{4}\right)^{2}$ (2.16). Then $n=i_{P}(1)$ by (2.16). Let $\psi \in z_{1} z_{2}+\left(z_{2}, z_{3}, z_{4}\right)^{2}$ be general enough so that $(\{\psi=0\}, 0)$ is an ordinary double point and $\{\phi=\psi=0\}$ is a surface smooth outside $z_{1}$-axis by Bertini's Theorem. Let $\phi_{\lambda}=\phi+\lambda \psi, X_{\lambda, \varepsilon}=\left\{(z)\left|\phi_{\lambda}(z)=0,\right| z_{i} \mid<\varepsilon(\forall i)\right\}$ and $C_{\lambda, \varepsilon}=X_{\lambda, \varepsilon} \cap C$ when $|\lambda| \ll \varepsilon \ll 1$. By (1b.4), $X_{\lambda, \varepsilon}$ has singularities only on $C_{\lambda, \varepsilon}$ if $|\lambda| \ll \varepsilon \ll 1$. Since $\phi_{\lambda} \equiv\left(z_{1}{ }^{n-1}+\lambda\right) z_{1} z_{2} \quad\left(z_{2}, z_{3}, z_{4}\right)^{2}$, one sees that $X_{\lambda, \varepsilon}$ has exactly $n$ singular points $\left\{(\xi, 0,0,0) \mid \xi^{n}+\lambda \xi=0\right\}$ in $C_{\lambda, \varepsilon}$ by solving 
$\partial \phi_{\lambda} / \partial z_{2}=0$ on $x_{1}$-axis. Again by $\phi_{\lambda} \equiv\left(z_{1}^{n-1}+\lambda\right) z_{1} z_{2}\left(z_{2}, z_{3}, z_{4}\right)^{2}, X_{\lambda, \varepsilon}$ has $c A$-points at these points, whence terminal (1a.2(ii)). If $n=1$, then $\left(X_{\lambda, \varepsilon}, 0\right)$ is an ordinary double point if $0<|\lambda| \ll 1$ because so is $(\{\psi=0\}, 0)$.

(4.7.4) To construct an $L$-smoothing for type (III) $P$, one may choose general $\psi \in z_{3}+\left(z_{2}, z_{3}, z_{4}\right)^{2}$ with the notation of (4.7.3), and the rest is similar to (4.7.3).

By using $L$-deformation (4.7), we can reduce many problems on extremal nbds to the ones on extremal nbds with only ordinary singularities. To derive formulae for ordinary singularities, we need an easy

(4.8) Lemma. Under the notation of (4.1), assume that

$$
I_{C^{\sharp}} / I_{C^{\sharp}}^{2}=\mathscr{O}_{C^{\sharp}} v_{1} \oplus \mathscr{O}_{C^{\sharp}} v_{2} \text { and } \omega_{X^{\sharp}} \otimes \mathscr{O}_{C^{\sharp}}=\mathscr{O}_{C^{\sharp}} \Omega
$$

near $P^{\sharp}$, where $I_{C^{\sharp}}$ is the defining ideal of $C^{\sharp}$ in $X^{\sharp}$, and $v_{1}, v_{2}$ and $\Omega$ are semi-invariants with $C^{\sharp}$-wts $b_{1}, b_{2}, k$. Then

$$
\begin{aligned}
\operatorname{gr}_{C}^{d} \mathscr{O} & =\bigoplus_{i, j \geq 0, i+j=d} \mathscr{O}_{C} t^{R\left(i b_{1}+j b_{2}\right)} v_{1}{ }^{i} v_{2}{ }^{j}, \\
\operatorname{gr}_{C}^{d} \omega & =\bigoplus_{i, j \geq 0, i+j=d} \mathscr{O}_{C} t^{R\left(i b_{1}+j b_{2}+k\right)} v_{1}{ }^{i} v_{2}{ }^{j} \Omega, \\
i_{P}(d) & ={ }_{m}^{1} \sum_{i, j \geq 0, i+j=d}\left\{i R\left(b_{1}\right)+j R\left(b_{2}\right)-R\left(i b_{1}+j b_{2}\right)\right\} \quad(d \geq 2), \quad \text { and } \\
w_{P}(d) & =\frac{1}{m} \sum_{i, j \geq 0, i+j=d}\left\{i R\left(b_{1}\right)+j R\left(b_{2}\right)+R(k)-R\left(i b_{1}+j b_{2}+k\right)\right\} \quad(d \geq 1) .
\end{aligned}
$$

Proof. By the assumptions, one has $I_{C^{\sharp}}=\left(v_{1}, v_{2}\right)$. Hence $I_{C^{\sharp}}^{(d)}=\left(v_{1}, v_{2}\right)^{d}$, $\mathrm{gr}_{C^{\sharp}}^{d} \mathscr{O} \simeq \bigoplus \mathscr{O}_{C^{\sharp}} v_{1}{ }^{i} v_{2}{ }^{j}$, and $\mathrm{gr}_{C^{\sharp}}^{d} \omega \simeq \bigoplus \mathscr{O}_{C^{\sharp}} v_{1}{ }^{i} v_{2}{ }^{j} \Omega$. The lemma follows from (2.8) and (2.9).

Then we have

(4.9) Theorem. Let $X \supset C \simeq \mathbb{P}^{1}$ be an extremal nbd, and let $P \in C$ be a singular point of $X$ of index $m \geq 1$, subindex $\bar{m}$, and splitting degree $s$. Let $(x)$ and $t$ be normalized $\ell$-coordinates as in (4.2), $a_{i}=\operatorname{ord}\left(x_{i}\right), \mathrm{ow}_{i}=\operatorname{ow}\left(x_{i}\right)$ $(i \in[1,4])$. Then

(i) the value of $w_{P}(0)$ is given depending on type of $P$ :

\begin{tabular}{c|c|c|c|c|c}
$w_{P}(0)$ & $\left(\bar{m}-a_{2}\right) / \bar{m}$ & $R\left(a_{4}\right) / \bar{m}$ & $3 / 4$ & $1 / 2$ & 0 \\
\hline type & $(\mathrm{IA}),\left(\mathrm{IA}^{\vee}\right)$ & $(\mathrm{IB}),(\mathrm{IC}),\left(\mathrm{IC}^{\vee}\right)$ & $(\mathrm{IIA}),(\mathrm{IIB})$ & $\left(\mathrm{II}^{\vee}\right)$ & $(\mathrm{III})$
\end{tabular}


(ii) if $P$ is ordinary, then depending on type of $P$, one has

Type (IA):

$$
\begin{aligned}
i_{P}(1) & =U\left(a_{1} a_{2}\right), \quad w_{P}(0)=\left(m-a_{2}\right) / m, \\
i_{P}(d) & =\sum_{i, j \geq 0, i+j=d}\left\{i U\left(a_{1} a_{2}\right)-U\left(i a_{1} a_{2}-j a_{1}\right)\right\} \quad(d \geq 2), \\
w_{P}^{*}(d) & =\sum_{i, j \geq 0, i+j=d} U\left(i a_{1} a_{2}-j a_{1}+a_{2}\right)-(d+1) \quad(d \geq 1),
\end{aligned}
$$

Type $\left(\mathrm{IA}^{\mathrm{v}}\right)$ :

$$
\begin{aligned}
& i_{P}(1)=U\left(s \cdot a_{1} a_{2}\right), \quad w_{P}(0)=\left(\bar{m}-a_{2}\right) / \bar{m}, \\
& i_{P}(d)=\sum_{i, j \geq 0, i+j=d}\left\{i U\left(s a_{2} \cdot \mathrm{ow}_{1}\right)-U\left(i s a_{2} \cdot \mathrm{ow}_{1}-j \cdot \mathrm{ow}_{1}\right)\right\} \quad(d \geq 2), \\
& w_{P}^{*}(d)=\sum_{i, j \geq 0, i+j=d} U\left(i s a_{2} \cdot \mathrm{ow}_{1}-j \cdot \mathrm{ow}_{1}+\mathrm{ow}_{2}\right)-(d+1) \quad(d \geq 1),
\end{aligned}
$$

Type (IC) or $\left(\mathrm{IC}^{\vee}\right)$ :

$$
\begin{array}{rlr}
i_{P}(1) & =U\left(s a_{1} a_{2}\right), \quad w_{P}(0)=R\left(a_{4}\right) / \bar{m}, \\
i_{P}(d) & =\sum_{i, j \geq 0, i+j=d}\left\{i U\left(s a_{1} a_{2}\right)+j U\left(a_{4}\right)-U\left(i s a_{1} a_{2}+j a_{4}\right)\right\} \quad(d \geq 2), \\
w_{P}^{*}(d) & =\sum_{i, j \geq 0, i+j=d}\left\{U\left(i s a_{1} a_{2}+(j+1) a_{4}\right)-(j+1) U\left(a_{4}\right)\right\} \quad(d \geq 1),
\end{array}
$$

and

Type (III):

$$
\begin{aligned}
i_{P}(1) & =1, \quad w_{P}(0)=0, \\
i_{P}(d) & =\left[d^{2} / 4\right] \quad(d \geq 2), \\
w_{P}^{*}(d) & =\left[(d+1)^{2} / 4\right] \quad(d \geq 1) .
\end{aligned}
$$

Proof. We omit the proof of (i) since it follows immediately from (2.10) for the case of type (IA) (resp. (IA $\left.{ }^{\vee}\right)$ ), where $R\left(a_{2}\right)=\bar{m}-a_{2}$ by $(\bar{m}, 0) \in \mathbb{Z}_{++} \mathrm{ow}_{1}+$ $\mathbb{Z}_{++} \mathrm{ow}_{2}$. We assume that $P$ is ordinary, and consider type (I) or $\left(I^{\vee}\right)$ case first. Let $F_{2}, F_{3}, F_{4}, J^{\sharp}$ be as in (4.3) (hence as in (2.11)). We use the notation of $\S 2$. We note $C^{\sharp}$-wt $\left(F_{i}\right) \equiv\left\|F_{i}\right\|(\bmod \bar{m}) \quad(i \in[2,4]) \quad$ (cf. $\left.(2.13)\right)$. Since $\phi$ is a part of basis for $J^{\sharp} / J^{\sharp 2}$, one has

$$
\begin{aligned}
{\left[\phi, J_{\{0\}}^{\sharp}, J_{\{0\}}^{\sharp}\right] } & =\left[J_{\{0\}}^{\sharp}, J_{\{0\}}^{\sharp}, J_{\{0\}}^{\sharp}\right] \\
& =\left[t^{R\left(\mathrm{wt} F_{2}\right)} F_{2}, t^{R\left(\mathrm{wt} F_{3}\right)} F_{3}, t^{R\left(\mathrm{wt} F_{4}\right)} F_{4}\right] \\
& =\bar{m} \sum_{i=2}^{4} U\left(\left\|F_{i}\right\|, \mathrm{wt} F_{i}\right)-\sum_{i=1}^{3} a_{i}
\end{aligned}
$$

by (2.8(ii)) and (2.14). Whence by (2.12), one has

$$
i_{P}(1)=1+\sum_{i=2}^{4} U\left(\left\|F_{i}\right\|, \mathrm{wt} F_{i}\right)-\frac{1}{\bar{m}}\left\{R(k)+\sum_{i=1}^{4} a_{i}\right\} \text {, }
$$


where $k=$ wt $x_{2}$ (resp. wt $\left.x_{4}\right)$ if $P$ is of type (IA) or (IA ${ }^{\vee}$ ) (resp. (IC) or $\left.\left(\mathrm{IC}^{\vee}\right)\right)$.

If $P$ is of type (IA) or $\left(\mathrm{IA}^{\vee}\right)$, we have $U\left(\left\|F_{2}\right\|\right.$, wt $\left.F_{2}\right)=U\left(s a_{2} \cdot\right.$ ow $\left._{1}\right)=$ $U\left(s a_{1} a_{2}\right), U\left(\left\|F_{4}\right\|\right.$, wt $\left.F_{4}\right)=U(\bar{m}, 0)=1$, and $U\left(\left\|F_{3}\right\|\right.$, wt $\left.F_{3}\right)=U\left(\right.$ ow $\left.x_{3}\right)=$ $\left(a_{1}+a_{3}\right) / \bar{m}$ by wt $x_{1}+$ wt $x_{3} \equiv 0(m)$ and $a_{1}<\bar{m}$. Since $(\bar{m}, 0) \in \mathbb{Z}_{++} \mathrm{ow}_{1}+$ $\mathbb{Z}_{++} \mathrm{ow}_{2}$, we have $(\bar{m}, 0)-\mathrm{ow}_{2} \in \mathrm{ow}\left(C^{\sharp}\right)$, and one has $R(k)+a_{2}=U\left(\mathrm{ow}_{2}\right)=$ $\bar{m}$, and thus $i_{P}(1)=U\left(s a_{1} a_{2}\right)$. The rest follows from (4.8) $\left(v_{1}=F_{2}, v_{2}=F_{3}\right)$ because $R(z)=U(z) \bar{m}-x \quad\left(z=(x, y) \in \mathrm{ow}\left(C^{\sharp}\right)\right)(2.8)$.

If $P$ is of type (IC) or $\left(\mathrm{IC}^{\vee}\right)$, we have $U\left(\left\|F_{2}\right\|\right.$, wt $\left.F_{2}\right)=U\left(s a_{1} a_{2}\right)$, $U\left(\left\|F_{3}\right\|\right.$, wt $\left.F_{3}\right)=U(\bar{m}, 0)=1$, and $U\left(\left\|F_{4}\right\|\right.$, wt $\left.F_{4}\right)=U\left(a_{4}\right)$ (we note $a_{4} \equiv$ wt $x_{4}(\bmod m)$ for type $\left(\mathrm{IC}^{\vee}\right)$ points). Whence we have $i_{P}(1)=U\left(s a_{1} a_{2}\right)$. The rest follows similarly from $(4.8)$ and $I_{C}=\left(F_{2}, F_{4}\right)(4.4(\mathrm{i}))$.

We consider type (III). Since $(X, P)$ is Gorenstein, $w_{P}(1)=0$ and $w_{P}(d)=$ $i_{P}(d) \quad(d \geq 2)$, whence $w_{P}^{*}(1)=i_{P}(1)$ and $w_{P}^{*}(d)=\left(\begin{array}{c}d+1 \\ 2\end{array}\right) i_{P}(1)-i_{P}(d)$ $(d \geq 2)$. We see $i_{P}(1)=1(2.16)$, and we need to calculate $i_{P}(d) \quad(d \geq 2)$. We may choose coordinates $\left(z_{1}, \ldots, z_{4}\right)$ so that $C$ is $z_{1}$-axis and $X=\left\{z_{1} z_{2}=\right.$ $\left.z_{3} z_{4}\right\}$ in $\left(\mathbb{C}^{4}, 0\right)$. Then $\mathscr{O}_{X, P}=\mathbb{C}\{z\} /\left(z_{1} z_{2}-z_{3} z_{4}\right)$, and let $I_{C}=\left(z_{2}, z_{3}, z_{4}\right)$, and $I_{C}{ }^{(d)}$ the symbolic $d$ th power of $I_{C}$. We note $z_{2} \in I_{C}{ }^{(2)}$ by $z_{1} z_{2}=z_{3} z_{4} \in$ $I_{C}{ }^{2}$. Let $J_{d}(d \in \mathbb{N})$ be the ideal generated by $z_{2}{ }^{i} z_{3}{ }^{j} z_{4}{ }^{k}$ for $i, j, k \in \mathbb{Z}_{+}$such that $2 i+j+k \geq d$. Then $I_{C} J_{d} \subset J_{d+1}$, and $J_{d} \subset I_{C}{ }^{(d)}$ by $z_{2} \in I_{C}^{(2)}$. By $z_{3} z_{4}=z_{1} z_{2}$, one sees that $v_{j k}:=z_{2}^{\min \{j, k\}} z_{3}^{j-\min \{j, k\}} z_{4}^{k-\min \{j, k\}} \in \mathscr{O}_{X, P}$ satisfies $z_{3}^{j} z_{4}^{k}=z_{1}^{\min \{j, k\}} v_{j k}$ for $j, k \in \mathbb{Z}_{+}$. Thus one easily sees that $J_{d}=$ $\left(v_{d, 0}, v_{d-1,1}, \ldots, v_{0, d}\right)$ and

$$
\bigoplus_{0 \leq i \leq d} \mathscr{O}_{C, P} v_{i, d-i} \rightarrow J_{d} / J_{d+1}
$$

Since $J_{1}=I_{C}$, we will see $J_{d}=I_{C}{ }^{(d)}$ by induction on $d$. Indeed if $J_{d}=I_{C}{ }^{(d)}$, then

$$
\bigoplus_{i=0}^{d} \mathscr{O}_{C, P} v_{i, d-i} \rightarrow\left(I_{C}^{(d)} / J_{d+1} \rightarrow I_{C}^{(d)} / I_{C}^{(d+1)}=\right) \mathrm{gr}_{C}^{d} \mathscr{O}
$$

is a surjection between free $\mathscr{O}_{C, P}$-modules of the same rank $d+1$, and hence it is an isomorphism and $J_{d+1}=I_{C}^{(d+1)}$. By $(*)$, one has

$$
\operatorname{gr}_{C}^{d} \mathscr{O} / S^{d} \operatorname{gr}_{C}^{1} \mathscr{O} \simeq \bigoplus_{i=0}^{d}\left(\mathscr{O}_{C, P} /\left(z_{1}^{\min \{i, d-i\}}\right)\right) \cdot v_{i, d-i}
$$


by $\operatorname{gr}_{C}^{1} \mathscr{O}=\mathscr{O}_{C, P} z_{3} \oplus \mathscr{O}_{C, P} z_{4}$ and $z_{3}{ }^{i} z_{4}{ }^{d-i}=z_{1}^{\min \{i, d-i\}} v_{i, d-i}$. Hence one sees $i_{P}(d)=\left[d^{2} / 4\right]$ and $w_{P}^{*}(d)=\left[(d+1)^{2} / 4\right](d \geq 2)$ by

$$
i_{P}(d)=\sum_{i=0}^{d} \min \{i, d-i\}= \begin{cases}2 \sum_{i=0}^{(d-1) / 2} i=\left(d^{2}-1\right) / 4 & \text { if } d \text { odd } \\ 2 \sum_{i=0}^{d / 2} i-d / 2=d^{2} / 4 & \text { if } d \text { even }\end{cases}
$$

We note that the formula for $w_{P}^{*}(d)$ works for $d=1$ as well.

(4.10) Remark. We note that $i_{P}(1)=\operatorname{siz}_{P}$ for ordinary $P$ by $(4.9(i i))$ (cf. (4.1.1)).

We can consider two slightly more delicate deformations than in (4.7).

(4.11) Lemma. Under the notation of (4.2), assume that $(X, P)$ is a cD-type terminal singularity of index 3 (1a.2) and that $P$ is a type (IA) point. Then $\left(a_{1}, \ldots, a_{4}\right)=(1,1,2,3)$ or $(2,1,1,3)$ and $w_{P}(0)=2 / 3$. Thus modulo permutation of $x_{1}$ and $x_{2}$ (4.2), we "may" assume $\left(a_{1}, \ldots, a_{4}\right)=(1,1,2,3)$.

Proof. By $m=3 \in \mathbb{Z}_{++} a_{1}+\mathbb{Z}_{++} a_{2}$, we see $a_{1}=1$ or $a_{2}=1$. If $a_{2}=1$, then $a_{1}+a_{3}=m=3$ by $\operatorname{ord}\left(C^{\sharp}\right)=\mathbb{Z}_{+}$and normalizedness, whence $\left(a_{1}, a_{3}\right)=(1,2)$ or $(2,1)$. We can also see $a_{2}=1,2$ and that $\left(a_{1}, a_{2}\right)=(1,2)$ if $a_{1}=1$. Thus it is enough to disprove $(a)=(1,2,2,3)$. Indeed it means that $\left.x_{1}\right|_{C^{\sharp}}=t$, $\left.x_{2}\right|_{C^{\sharp}}=\left.x_{3}\right|_{C^{\sharp}}=t^{2}$, and $\left.x_{4}\right|_{C^{\sharp}}=t^{3}$. By (1a.6), $\phi \in\left(x_{4}\right)^{2}+x_{4}\left(x_{1}, x_{2}, x_{3}\right)^{2}+$ $\left(x_{1}, x_{2}, x_{3}\right)^{3}$ and $x_{1}{ }^{3}$ appears in $\phi$ because wt $x_{1} \not \equiv$ wt $x_{2}$, wt $x_{3}$. Hence $\left.\phi\right|_{C}$ is of order 3 , which is a contradiction. Thus $(a)=(1,1,2,3)$ or $(2,1,1,3)$, whence $w_{P}(0)=2 / 3$ by $(4.9(\mathrm{i}))$.

(4.12) Under the notation and assumptions of (4.2), assume that $P$ is a type (IA) point with $a_{1}=1$ or a type (IIA) point. Then there exists an $\ell$-coordinate system $(z)=\left(z_{1}, \ldots, z_{4}\right)$ for $(X, P)$ and an $\ell$-coordinate for $(C, P)$ such that $C^{\sharp}$ is the $z_{1}$-axis, wt $z_{i} \equiv a_{i}(i \in[1,4])$, and $\phi \equiv z_{1}{ }^{n} z_{q}\left(z_{2}, z_{3}, z_{4}\right)^{2}$, where $n=\operatorname{len}_{P^{\sharp}} I_{C^{\sharp}}{ }^{(2)} / I_{C^{\sharp}}{ }^{2} \geq 0$ and $q \in[2,4](2.16)$.

We note that if $P$ is of type (IA) and $n \geq 2$, then wt $z_{2} z_{3} \equiv 0(m)$. Because otherwise, we see that $m \geq 3$ and $\phi \in \mathbb{C} z_{4}{ }^{2}+(z)^{3}$, hence $(X, P)$ is a $c D$-type terminal singularity of index 3 (1a.6) and wt $z_{2} z_{3} \equiv 0$ by (4.11), which is a contradiction. Thus we have

(4.12.1) Sublemma. If $P$ is a type (IA) point with $a_{1}=1$ and $\operatorname{len}_{P^{\sharp}} I_{C^{\sharp}}^{(2)} / I_{C^{\sharp}}^{2}$ $\geq 2$, then $a_{2}=1$.

If $P$ is of type (IA) and $n \geq m$ (resp. $P$ is of type (IIA)), then we can choose a general semi-invariant

$$
\psi \in \mathbb{C}^{*} z_{1}{ }^{m-a_{q}} z_{q}+\mathbb{C}^{*} z_{2} z_{3}+I_{C^{\sharp}}{ }^{3} \quad\left(\text { resp. } \psi \in \mathbb{C}^{*} z_{1} z_{2}+\mathbb{C}^{*} z_{4}{ }^{3}+I_{C^{\sharp}}{ }^{4}\right)
$$


such that $\{\phi=\psi=0\}$ is a surface which is smooth outside $z_{1}$-axis by Bertini's Theorem. Then as in (4.6) and (4.7), $\phi_{\lambda}=\phi+\lambda \psi$ induces a deformation $X_{\lambda, \varepsilon} \supset C_{\lambda, \varepsilon} \ni P$ of nbd of $P$ in $X \supset C$ such that $X_{\lambda, \varepsilon} \supset C_{\lambda, \varepsilon}$ is locally primitive, and nearby extremal nbds $X_{\lambda}^{\circ} \supset C_{\lambda}$.

(4.12.2) Proposition-Definition. (i) If $P$ is a type (IA) point with $a_{1}=1$ and $n=\operatorname{len}_{P^{\sharp}} I_{C^{\sharp}}{ }^{(2)} / I_{C^{\sharp}}{ }^{2} \geq m$, then $X_{\lambda, \varepsilon} \supset C_{\lambda, \varepsilon}$ has a type (IA) point with index $m$ and at least one type (III) point, and

(ii) if $P$ is of type (IIA), then $X_{\lambda} \supset C_{\lambda} \ni P$ has an $\ell$-coordinate system (y) (depending on $\lambda$ ) such that wt $y_{i} \equiv a_{i}(i \in[1,4]), C_{\lambda}^{\sharp}$ is the $y_{1}$-axis, $\phi_{\lambda}=y_{1} y_{2}+y_{3}{ }^{2}+y_{4}{ }^{3}$ for each small enough $\lambda>0$.

We call all these processes ( or $X_{\lambda}^{\circ} \supset C_{\lambda}$, by abuse of language) $L^{\prime}$-deformations at $P$.

Proof. Since $S^{\sharp}=\operatorname{Sing}\{\phi=\psi=0\}$ is contained in $z_{1}$-axis, $S^{\sharp} \cap F^{\sharp} \subset\{0\}$, where $F^{\sharp}$ is the fixed axis, i.e. $z_{4}$-axis (1a.5).

(i) Since $z_{2} z_{3}$ appears in $\psi$, the conditions in (1b.3) are satisfied by (1b.5), and (1b.3) applies. The argument is similar to (4.7), and we only show existence of an extra type (III) point. By

$$
\phi_{\lambda} \equiv\left(z_{1}^{n-\left(m-a_{4}\right)}+\lambda\right) z_{1}^{m-a_{q}} z_{q} \bmod \left(z_{2}, z_{3}, z_{4}\right)^{2},
$$

we see a moving singular point by $n-\left(m-a_{q}\right)>0$. This gives a type (III) point since it is not on the fixed axis (1b.3).

(ii) By (1a.5), $z_{3}{ }^{2}$ appears in $\phi$, whence the quadratic part of $\phi_{\lambda}$ is of the form $\alpha z_{1} z_{2}+\beta z_{2}{ }^{2}+\gamma z_{3}{ }^{2}(\alpha, \beta, \gamma \in \mathbb{C}, \alpha \gamma \neq 0)$. Thus using the Weierstrass Preparation Theorem, one can easily obtain an $\ell$-coordinate system $(y)$ such that wt $y_{i} \equiv a_{i} \quad(i \in[1,4]), C_{\lambda}^{\sharp}$ is the $y_{1}$-axis, $\phi_{\lambda}=y_{1} y_{2}+y_{3}{ }^{2}+y_{4}{ }^{2 r+1}$ $(r \geq 1)$. By construction of $\psi,\left.\phi\right|_{F^{\sharp}}$ has exactly a triple root at the origin, and we note that $F^{\sharp}=y_{4}$-axis in terms of the coordinates $y$. Thus $r=1$ (1a.5(iii)). By (1a.7(ii)), $\left(\left\{\phi_{\lambda}=0\right\}, 0\right) / \mu_{m}$ is terminal. Thus the conditions in (1b.3) are satisfied and the rest is the same.

5. Numerical Calculations for (IA), (IC), (IA $\left.{ }^{\vee}\right)$, AND $\left(I^{\vee}\right)$

The main purpose of this section is to make formulae (4.9) more explicit for later use. Throughout this section, we will use the notation of (4.9).

(5.1) Proposition. Assume that $P$ is an ordinary point of type (IA) with index $m$ such that $i_{P}(1)=1, w_{P}(0)<1 / 2$. Then

(i) one has $a_{1}=1$, and 
(ii) if $d \in \mathbb{N}$ satisfies $w_{P}(0)<1 / d$, then one has (we note $d \leq m-1$ and $1 /(d+1) \leq(m-1) / m d<1 / d)$

$$
\begin{aligned}
\left(i_{P}(d+1), w_{P}^{*}(d)\right) \\
\quad= \begin{cases}\left(0, \frac{1}{2} d(d+1)\right) & \text { if } w_{P}(0)<\frac{1}{d+1}, \\
\left(1, \frac{1}{2} d(d+1)-1\right) & \text { if } w_{P}(0) \geq \frac{1}{d+1}, w_{P}(0) \neq \frac{m-1}{m \cdot d}, \\
\left(2, \frac{1}{2} d(d+1)-2\right) & \text { if } w_{P}(0)=\frac{m-1}{m \cdot d}, d \neq m-1, \\
\left(d+2, \frac{1}{2}(d-2)(d+1)\right) & \text { if } w_{P}(0)=\frac{1}{m}, d=m-1 .\end{cases}
\end{aligned}
$$

Proof. One has $a_{1} a_{2} \leq m$ by $U\left(a_{1} a_{2}\right)=1$ and $a_{2}>m / 2$ by $w_{P}(0)=$ $\left(m-a_{2}\right) / m<1 / 2(4.9)$. Thus $a_{1}=1$ and $\operatorname{ord}\left(C^{\sharp}\right)=\mathbb{Z}_{+}$, which proves (i). Since $w_{P}(0) \geq 1 / m$, one has $d<m$ and $1 /(d+1) \leq(m-1) / m d<1 / d$ is immediately checked. We note that $U(n)=\lceil n / m\rceil$ for $n \in \mathbb{Z}$ by $\operatorname{ord}\left(C^{\sharp}\right)=\mathbb{Z}_{+}$. Then $r=m-a_{2} \in \mathbb{N}$ satisfies $d r<m$ by $w_{P}(0)=r / m<1 / d$. Let us consider arbitrary $i, j \in \mathbb{Z}_{+}$such that $i+j=d+1$. Then we claim

$$
U\left(i a_{2}-j\right)= \begin{cases}i-1 & \text { if }\left\{\begin{array}{ll}
j=0 & \text { and } w_{P}(0) \geq \frac{1}{d+1}, \\
j=1 & \text { and } w_{P}(0)=\frac{m-1}{m \cdot d}, \text { or } \\
j>1 & \text { and } w_{P}(0)=\frac{1}{m} \text { and } d=m-1,
\end{array}\right. \text { otherwise. }\end{cases}
$$

Indeed from

$$
m i-\left(i a_{2}-j\right)=\left(m-a_{2}\right) i+j>0,
$$

and

$$
\begin{aligned}
\left(i a_{2}-j\right)-m(i-2) & =2 m-j-i\left(m-a_{2}\right) \\
& =2 m-j-i r \\
& \geq 2 m-(d+1) r>0,
\end{aligned}
$$

one has $i \geq U\left(i a_{2}-j\right) \geq i-1$ and $U\left(i a_{2}-j\right)=i-1$ iff $X(i, j) \leq 0$, where $X(i, j)=i\left(a_{2}-j\right)-m(i-1)=m-j-i r$. It is clear that $X(d+1,0) \leq 0 \Leftrightarrow$ $m \leq(d+1) r \Leftrightarrow w_{P}(0) \geq 1 /(d+1)$. If $j \geq 1$, then

$$
\begin{aligned}
X(i, j) & =m-1-(j-1)-i r \\
& \geq m-1-(j-1) r-i r \\
& =m-1-d r \geq 0 .
\end{aligned}
$$

One sees that $X(d, 1) \leq 0$ iff $m-1=d r$, that is $w_{P}(0)=(m-1) / m d$. If $j>1$, then $X(i, j) \leq 0$ iff $m-1=d r$ and $r=1$, that is $w_{P}(0)=1 / m$ and 
$d=m-1$. Thus the claim is proved. Since (4.9) implies

$$
\begin{aligned}
i_{P}(d+1) & =\sum\left\{i-U\left(i a_{2}-j\right)\right\}, \quad \text { and } \\
w_{P}^{*}(d) & =\sum_{i \geq 1} U\left(i a_{2}-j\right)-(d+1) \\
& =\sum i-\sum\left\{i-U\left(i a_{2}-j\right)\right\}-U(-d-1)-(d+1) \\
& =\frac{1}{2} d(d+1)-i_{P}(d+1)-U(-d-1)
\end{aligned}
$$

the above claim implies (5.1).

(5.2) Proposition. Assume that $P$ is an ordinary point of type (IA) and index $m$. One has

(i) $i_{P}(1)=1$ and $a_{2}>1 \Rightarrow i_{P}(2) \leq 1$,

(ii) $a_{2}>1 \Rightarrow w_{P}^{*}(1) \geq i_{P}(1)-1$, and

(iii) $w_{P}^{*}(1) \geq-1$, where “=" holds iff $a_{2}=1\left(\Leftrightarrow w_{P}(0)=(m-1) / m\right)$. If furthermore $a_{2}=1$, then

$$
\begin{aligned}
i_{P}(d) & =\frac{1}{2} d(d+1)-\left\lceil\frac{d}{2}\right\rceil+\sharp S(d) \quad(d \geq 2), \\
w_{P}^{*}(d) & =-\left\lceil\frac{d}{2}\right\rceil+\sharp S(d) \quad(d \geq 1),
\end{aligned}
$$

where $S(d)=\{i \in \mathbb{Z} \mid 0<i \leq d, i \equiv d(2), i \equiv 0 \quad(m)\}$ (we note $\sharp S(d)=\delta_{d, m}$ if $\left.d \leq m\right)$.

Proof. Assume that $a_{2}>1$. Let $m=\alpha a_{1}+\beta a_{2} \quad(\alpha, \beta \in \mathbb{N})$. By $\left(a_{1}, m\right)=1$, one sees $\left(a_{1}, a_{2}\right)=1$. Thus $a_{1}-a_{2} \notin \mathbb{Z}_{+} a_{1}+\mathbb{Z}_{+} a_{2}$ and $m+a_{1}-a_{2}=$ $(\alpha+1) a_{1}+(\beta-1) a_{2} \in \mathbb{Z}_{+} a_{1}+\mathbb{Z}_{+} a_{2}$, whence $U\left(a_{2}-a_{1}\right)=1$. Hence

$$
\begin{aligned}
w_{P}^{*}(1) & =U\left(a_{1} a_{2}+a_{2}\right)+U\left(a_{2}-a_{1}\right)-2 \\
& =U\left(a_{1} a_{2}+a_{2}\right)-1 \geq U\left(a_{1} a_{2}\right)-1 \geq i_{P}(1)-1,
\end{aligned}
$$

which proves (ii). One sees $-m-\left(-2 a_{1}\right)=2 a_{1}-m=(2-\alpha) a_{1}-\beta a_{2} \notin$ $\mathbb{Z}_{+} a_{1}+\mathbb{Z}_{+} a_{2}$ by $2-\alpha \leq 1$ and $\beta>0$ (note $\left(a_{1}, a_{2}\right)=1$ and $a_{2}>1$ ). Thus $U\left(-2 a_{1}\right)=0$. Let us assume $i_{P}(1)=U\left(a_{1} a_{2}\right)=1$ to prove (i). Then $U\left(a_{1} a_{2}-a_{1}\right)=1$ by $a_{1} a_{2}-a_{1}>0$ and $U\left(a_{1} a_{2}\right) \geq U\left(a_{1} a_{2}-a_{1}\right) \geq 1$. One has $2=2 U\left(a_{1} a_{2}\right) \geq U\left(2 a_{1} a_{2}\right) \geq U\left(a_{1} a_{2}\right)=1$. Thus one has $i_{P}(2)=2 U\left(a_{1} a_{2}\right)-$ $U\left(2 a_{1} a_{2}\right)+U\left(a_{1} a_{2}\right)-U\left(a_{1} a_{2}-a_{1}\right)-U\left(-2 a_{1}\right)=0$ or 1 , which proves (i). By (ii) and (2.15), one sees $w_{P}^{*}(1) \geq 0$ if $a_{2}>1$. The formula in (iii) implies $w_{P}^{*}(1)=-1$ if $a_{2}=1$. We now need to prove the formulae in (iii) assuming $a_{2}=1$. Then $\operatorname{ord}\left(C^{\sharp}\right)=\mathbb{Z}_{+}, U(n)=\lceil n / m\rceil$ for $n \in \mathbb{Z}$, and $U\left(a_{1}, a_{2}\right)=1$. Hence (4.9) implies

$$
\begin{aligned}
i_{P}(d) & =\sum_{i, j \geq 0, i+j=d}\left(i-\left\lceil\frac{(i-j) a_{1}}{m}\right\rceil\right) \\
& =\frac{1}{2} d(d+1)-\sum_{0<i \leq d, i \equiv d(2)}\left(\left\lceil\frac{i a_{1}}{m}\right\rceil+\left\lceil-\frac{i a_{1}}{m}\right\rceil\right)
\end{aligned}
$$


for $d \geq 2$. By

$$
2=2+\frac{x}{m}+\frac{-x}{m}>\left\lceil\frac{x}{m}\right\rceil+\left\lceil\frac{-x}{m}\right\rceil \geq \frac{x}{m}+\frac{-x}{m}=0
$$

one sees

$$
\left\lceil\frac{x}{m}\right\rceil+\left\lceil\frac{-x}{m}\right\rceil= \begin{cases}1 & \text { if } x \neq 0(m) \\ 0 & \text { otherwise }\end{cases}
$$

whence formula for $i_{P}(d)$ follows. One also has

$$
\begin{aligned}
w_{P}^{*}(d) & =\sum_{i, j \geq 0, i+j=d}\left(\left\lceil\frac{(i-j) a_{1}+1}{m}\right\rceil-1\right) \\
& =\sum_{0<i \leq d, i \equiv d(2)}\left(\left\lceil\frac{i a_{1}+1}{m}\right\rceil+\left\lceil\frac{-i a_{1}+1}{m}\right\rceil-2\right)
\end{aligned}
$$

for $d \geq 1$. It is clear that

$$
1+\frac{x}{m}=\frac{m-1}{m}+\frac{x+1}{m} \geq\left\lceil\frac{x+1}{m}\right\rceil \geq \frac{x+1}{m} \quad(x \in \mathbb{Z})
$$

and that the first inequality is an inequality iff $x \equiv 0(m)$. Hence from

$$
2=2+\frac{x}{m}+\frac{-x}{m} \geq\left\lceil\frac{1+x}{m}\right\rceil+\left\lceil\frac{1-x}{m}\right\rceil \geq \frac{1+x}{m}+\frac{1-x}{m}=\frac{2}{m},
$$

one sees that

$$
2 \geq\left\lceil\frac{1+x}{m}\right\rceil+\left\lceil\frac{1-x}{m}\right\rceil \geq 1
$$

and the first inequality is an equality iff $x \equiv 0(m)$. Then the formula for $w_{P}^{*}(d)$ follows.

There is another case of (IA) with $i_{P}(1)=1$, in which we need to calculate $i_{P}(d)$ for large $d$.

(5.3) Proposition. Assume that $P$ is an ordinary point of type (IA) with index $m$. If $w_{P}(0)=1 / m$, then $a_{1}=1, a_{2}=m-1, i_{P}(1)=1$, and

$$
\begin{aligned}
i_{P}(d) & =(d+1)\left[\frac{d}{m}\right] \quad(d \geq 2), \\
w_{P}^{*}(d) & =\frac{1}{2}(d+1)\left\{d-2\left[\frac{d+1}{m}\right]\right\} \quad(d \geq 1) .
\end{aligned}
$$

Proof. By $w_{P}(0)=1 / m$, one has $a_{2}=m-1$ (4.9). Thus by $m \in \mathbb{Z}_{++} a_{1}+$ $\mathbb{Z}_{++} a_{2}$, one has $a_{1}=1$. Hence $i_{P}(1)=1, \operatorname{ord}\left(C^{\sharp}\right)=\mathbb{Z}_{+}$, and $U(n)=\lceil n / m\rceil$ for $n \in \mathbb{Z}$. Then (4.9) implies

$$
\begin{aligned}
i_{P}(d) & =\sum\{i-U(i m-d)\}=\sum\left[\frac{d}{m}\right]=(d+1)\left[\frac{d}{m}\right] & (d \geq 2), \\
w_{P}^{*}(d) & =\sum U((i+1) m-(d+1))-(d+1) & \\
& =\sum\left(i-\left[\frac{d+1}{m}\right]\right)=\frac{1}{2}(d+1)\left\{d-2\left[\frac{d+1}{m}\right]\right\} & (d \geq 1) .
\end{aligned}
$$


The following provides us with necessary information for our calculation in (6.5).

(5.4) Proposition. Assume that $P$ is an ordinary point of type (IA) with index $m$ such that $i_{P}(1)=2$ and $w_{P}(0)<1 / 2$. Let $a_{1}, a_{2}$ be as in the definition of type (IA) in (4.2). Then

(i) $a_{1}=2, a_{2} \geq(m+1) / 2, a_{2}, m \equiv 1$ (2), and

(ii)

$$
w_{P}^{*}(1) \geq 2, \quad w_{P}^{*}(2) \geq \begin{cases}5 & \text { if } w_{P}(0)<2 / 5, \\ 4-\delta_{a_{2},(m+1) / 2} & \text { if } w_{P}(0) \geq 2 / 5,\end{cases}
$$

and furthermore if $w_{P}^{*}(2)=3$, then $U\left((2 i+1) a_{2}-2(2-i)\right)=i+1$ for $i \in[0,2] \quad(c f .(2.8))$.

Proof. By $w_{P}(0)=\left(m-a_{2}\right) / m<1 / 2(4.9)$, one has $a_{2}>m / 2$. From $i_{P}(1)=$ $U\left(a_{1} a_{2}\right)=2$ (4.9), one sees $2 m \geq a_{1} a_{2}>a_{1} m / 2$, whence $a_{1} \leq 3$. Since $a_{1} \geq 2$ by $U\left(a_{1} a_{2}\right)=2$, one will see $a_{1}=2$. Indeed if $a_{1}=3$, then $2 m-3 a_{2}$ $\left(<a_{2}\right)$ belongs to $3 \mathbb{Z}_{+}+a_{2} \mathbb{Z}_{+}$, whence $2 m-3 a_{2} \equiv 0$ (3), which however contradicts $(m, 3)=1$. Hence $a_{1}=2$ and $m, a_{2} \equiv 1$ (2). This proves (i).

By $m \in \mathbb{Z}_{++} 2+\mathbb{Z}_{++} a_{2}$ and $a_{2}>m / 2$, one sees $m \geq 5$ and $m=a_{2}+2 r$ for some $r \in \mathbb{N}$ such that $2 r<a_{2}$. Let $i, j \in \mathbb{Z}_{+}$be such that $i+j \leq 2$. Then we claim

(5.4.1) $U\left((2 i+1) a_{2}-2 j\right) \geq i+1$, where

$$
“=" \Leftrightarrow\left\{\begin{array}{l}
i=0, \text { or } \\
(i+1) r+j \geq a_{2} .
\end{array}\right.
$$

To see this, let $X(\alpha, i, j)=(i+\alpha) m-\left\{(2 i+1) a_{2}-2 j\right\}$ for $\alpha \in \mathbb{Z}_{+}$. We note that $U\left((2 i+1) a_{2}-2 j\right) \leq i+\alpha$ iff $X(\alpha, i, j) \in \mathbb{Z}_{+} 2+\mathbb{Z}_{+} a_{2}$ by definition of $U()$, and that

$$
X(0, i, j)=-(i+1) a_{2}+2(i r+j)=(1-i) a_{2}+2\left(i r+j-a_{2}\right)
$$

by $m=a_{2}+2 r$. Since $\left(a_{2}, 2\right)=1$ and $i r+j-a_{2} \leq 2 r-a_{2}<0$, this means that $X(0, i, j) \notin \mathbb{Z}_{+} a_{2}+2 \mathbb{Z}_{+}$and $U\left((2 i+1) a_{2}-2 j\right) \geq i+1$. Then

$$
\begin{aligned}
X(1, i, j) & =X(0, i, j)+m=-i a_{2}+2((i+1) r+j) \\
& =(2-i) a_{2}+2\left((i+1) r+j-a_{2}\right)
\end{aligned}
$$

by $m=a_{2}+2 r$. Then $X(1,0, j)=2(r+j) \in \mathbb{Z}_{+} 2$ and thus $U\left((2 i+1) a_{2}-2 j\right)=$ $i+1$ if $i=0$. Assume that $i>0$, i.e. $i=1,2$. Then one similarly sees by (5.4.2) that $X(1, i, j) \in \mathbb{Z}_{+} a_{2}+2 \mathbb{Z}_{+}$iff $(i+1) r+j-a_{2} \geq 0$. Thus our claim (5.4.1) is proved. By (5.4.1) and $a_{2}>2 r$, one sees $U\left(3 a_{2}\right) \geq 3$ and $U\left(a_{2}-2\right)=1$, whence

$$
w_{P}^{*}(1)=U\left(3 a_{2}\right)+U\left(a_{2}-2\right)-2 \geq 2
$$


by $\left(4.9\right.$ (ii)). Similarly, one sees $U\left(a_{2}-4\right)=1$, and

$$
\begin{aligned}
& U\left(3 a_{2}-2\right) \geq 2, \quad \text { where } "=" \Leftrightarrow 2 r+1=a_{2}, \text { i.e. } a_{2}=(m+1) / 2, \\
& U\left(5 a_{2}\right) \geq 3, \quad \text { where } "=" \Leftrightarrow 3 r \geq a_{2}, \text { i.e. } w_{P}(0) \geq 2 / 5
\end{aligned}
$$

because $2 r+1 \leq a_{2}$ and $a_{2}=m-2 r$. By (4.9(ii)), one has

$$
w_{P}^{*}(2)=U\left(5 a_{2}\right)+U\left(3 a_{2}-2\right)+U\left(a_{2}-4\right)-3 .
$$

If $w_{P}(0)<2 / 5$, then $a_{2}>3 m / 5 \geq(m+1) / 2$ by $m \geq 5$. Hence $w_{P}^{*}(2) \geq 5$ if $w_{P}(0)<2 / 5$, and the estimate for $w_{P}^{*}(2)$ follows immediately. If $w_{P}^{*}(2)=3$, then the above argument shows $U\left(5 a_{2}\right)=3, U\left(3 a_{2}-2\right)=2, U\left(a_{2}-4\right)$ $=1$.

(5.5) Proposition. Assume that $P$ is of type (IC) with index $m$. Then one has

(i) $i_{P}(1)=a_{1}(\geq 2$ by (4.2)),

(ii)

$$
w_{P}^{*}(1)=\left\{\begin{array}{l}
i_{P}(1) \quad \text { if } w_{P}(0) \neq(m-1) / m, \\
i_{P}(1)-\delta_{a_{1}, 2}-\delta_{a_{1},(m-1) / 2} \text { otherwise, }
\end{array}\right.
$$

(iii) if $i_{P}(1)=2$ and $w_{P}(0) \neq(m-1) / m$, then $i_{P}(2)=0$,

(iv) if $d \in \mathbb{N}$ satisfies $w_{P}(0)<1 / d \leq 1 / 2$, then $i_{P}\left(d^{\prime}+1\right)=0$ and $w_{P}^{*}\left(d^{\prime}\right)=$ $a_{1} d^{\prime}\left(d^{\prime}+1\right) / 2$ for all $d^{\prime} \in[1, d]$, and

(v) if $w_{P}(0)=4 / 5$ and $m=5$, then $w_{P}^{*}(2)=0$ and $w_{P}^{*}(3)=4$.

Proof. By $w_{P}(0)=R\left(a_{4}\right) / m<1, R\left(a_{4}\right) \quad(<m)$ belongs to $\mathbb{Z}_{+} a_{1}+\mathbb{Z}_{+} a_{2}$. By $a_{4} \not \equiv a_{1}, a_{2}(m), a_{1}+a_{2}=m, 2 \leq a_{1}<a_{2}$, one sees $m \geq 5$ and $R\left(a_{4}\right)=r a_{1}$ for some $r \in\left[2, m / a_{1}\right)$. Let $u=U\left(a_{4}\right)$, whence $a_{4}=u m-r a_{1}$. Let us choose $d \in \mathbb{N}$ such that $w_{P}(0)<1 / d$, i.e. $r a_{1} d<m$. For $i, j \in \mathbb{Z}_{+}$such that $i+j \leq d+1$, we claim

(5.5.1) $U\left(i a_{1} a_{2}+j a_{4}\right)=i a_{1}+j u-\operatorname{Max}\left\{0, i a_{1}+r j-a_{2}\right\}$.

First we note

$$
\begin{aligned}
i a_{1}+r j-m & <i a_{1}+r j-r a_{1} d \\
& \leq(d+1) \cdot \operatorname{Max}\left\{a_{1}, r\right\}-r a_{1} d \\
& =\operatorname{Max}\left\{a_{1}, r\right\}\left(d+1-d \cdot \operatorname{Min}\left\{a_{1}, r\right\}\right) \leq 0
\end{aligned}
$$

by $a_{1}, r \geq 2$. For $\alpha \geq 0$, let $X(\alpha)=\left(i a_{1}+j u-\alpha\right) m-\left(i a_{1} a_{2}+j a_{4}\right)$ so that $U\left(i a_{1} a_{2}+j a_{4}\right) \leq i a_{1}+j u-\alpha$ iff $X(\alpha) \in \mathbb{Z}_{+} a_{1}+\mathbb{Z}_{+} a_{2}$. Then

$$
\begin{aligned}
X(\alpha) & =i a_{1}\left(m-a_{2}\right)+j\left(u m-a_{4}\right)-\alpha m \\
& =i a_{1}^{2}+j r a_{1}-\alpha\left(a_{1}+a_{2}\right) \\
& =\left(i a_{1}+r j-\alpha\right) a_{1}-\alpha a_{2} \\
& =\left(i a_{1}+r j-a_{2}-\alpha\right) a_{1}+\left(a_{1}-\alpha\right) a_{2} .
\end{aligned}
$$

Thus $X(0) \in \mathbb{Z}_{+} a_{1}$ and $U\left(i a_{1} a_{2}+j a_{4}\right) \leq i a_{1}+j u$. For $\alpha>0$, one has $i a_{1}+r j-a_{2}-\alpha \leq m-a_{2}-\alpha=a_{1}-\alpha<a_{1}<a_{2}$ by (5.5.2). Thus by $\left(a_{1}, a_{2}\right)=1$, one sees $X(\alpha) \in \mathbb{Z}_{+} a_{1}+\mathbb{Z}_{+} a_{2}$ iff $i a_{1}+r j-a_{2}-\alpha \geq 0$. Hence 
$\alpha=\operatorname{Max}\left\{0, i a_{1}+r j-a_{2}\right\}$ is the largest $\alpha \in \mathbb{Z}_{+}$such that $X(\alpha) \in \mathbb{Z}_{+} a_{1}+\mathbb{Z}_{+} a_{2}$, whence (5.5.1) follows. Setting $(i, j)=(1,0)$, one has $U\left(a_{1} a_{2}\right)=a_{1}$ and $(\mathrm{i})$ follows. Using $m \geq r a_{1}+1$ and setting $d=1$, one sees

$$
\begin{aligned}
i a_{1}+r j-a_{2} & =(i+1) a_{1}+r j-m \\
& \leq(i+1) a_{1}+r j-1-r a_{1} \\
& =-\left(a_{1}-j\right)(r-i-1)+j(i+1)-1 .
\end{aligned}
$$

For $(i, j)=(1,1)($ resp. $(0,2))$, one sees $j(i+1)=2, a_{1} \geq j, r \geq i+1$. Thus

$$
i a_{1}+r j-a_{2} \leq 1
$$

and the equality holds iff $m=r a_{1}+1$ and $r=2$ (resp. $a_{1}=2$ ), i.e. iff $a_{4} \equiv 1$ $(m)$ and $a_{1}=(m-1) / 2$ (resp. $\left.a_{1}=2\right)$. This is because $r=2 \Leftrightarrow a_{1}=(m-1) / 2$ when $m=r a_{1}+1$. Thus by (4.9) and (5.5.1), one has

$$
\begin{aligned}
w_{P}^{*}(1) & =\sum_{(i, j)=(1,1),(0,2)}\left\{U\left(i a_{1} a_{2}+j a_{4}\right)-j u\right\} \\
& =\left\{\begin{array}{l}
a_{1}-\delta_{a_{1}, 2}-\delta_{a_{1},(m-1) / 2} \\
a_{1} \text { otherwise, }
\end{array}\right.
\end{aligned}
$$

whence follows (ii). By (5.5.1) with $(i, j)=(2,0)$, one has $U\left(2 a_{1} a_{2}\right)=2 a_{1}-$ $\operatorname{Max}\left\{0,3 a_{1}-m\right\}$, whence one similarly obtains

$$
i_{P}(2)= \begin{cases}\operatorname{Max}\left\{0,3 a_{1}-m\right\}+\delta_{a_{1}, 2}+\delta_{a_{1},(m-1) / 2} & \text { if } a_{4} \equiv 1(m), \\ \operatorname{Max}\left\{0,3 a_{1}-m\right\} & \text { otherwise. }\end{cases}
$$

If $i_{P}(1)=2$ and $w_{P}(0) \neq(m-1) / m$, then $a_{1}=2$ and $a_{4} \not \equiv 1(m)$ by (i), whence $m \geq 2 r+2 \geq 6$ and $i_{P}(2)=0$. Hence follows (iii). When $d \geq 2$, one sees

$$
\begin{aligned}
i a_{1}+r j-a_{2} & \leq i a_{1}+r j-m+\operatorname{Max}\left\{a_{1}, r\right\} \\
& <\operatorname{Max}\left\{a_{1}, r\right\}\left(d+2-d \cdot \operatorname{Min}\left\{a_{1}, r\right\}\right) \leq 0
\end{aligned}
$$

by $a_{1}, r \geq 2$ and (5.5.2). Thus by (5.5.1), one sees

$$
i_{P}\left(d^{\prime}+1\right)=0 \text { and } w_{P}^{*}\left(d^{\prime}\right)=a_{1} d^{\prime}\left(d^{\prime}+1\right) / 2 \text { for } d^{\prime} \in[1, d]
$$

if $d \geq 2$. This proves (iv). Assume that $m=5$ and $a_{4} \equiv 1$ (5). Then $a_{1}=r=2, a_{2}=3, u=2$, and $a_{4}=6$. Thus $\mathbb{Z}_{+} a_{1}+\mathbb{Z}_{+} a_{2}=\mathbb{Z}_{+}-\{1\}$. Hence for $x, y, z \in \mathbb{Z}_{+}$such that $x+y=z$, one has $U\left(x a_{1} a_{2}+(y+1) a_{4}\right)=U(6 z+6)$. Thus by (4.9), one has

$$
\begin{aligned}
w_{P}^{*}(z) & =(z+1) \cdot U(6 z+6)-(z+1)(z+2)=(z+1) \cdot U(z-4) \\
& =(z+1)\left(\left\lceil\frac{z-4}{5}\right\rceil+\delta_{(z \bmod 5), 3}\right)
\end{aligned}
$$

where $\delta_{(x \bmod 5), y}$ denotes 1 if $x \equiv y(5)$ and 0 otherwise. This proves $(\mathrm{v})$.

The calculation needed for imprimitive points is much simpler. 
(5.6) Proposition. Let $P$ be an ordinary point of type $\left(\mathrm{IA}^{\vee}\right)$. Then we have

(i) $w_{P}^{*}(1) \geq i_{P}(1)-1$, and

(ii) $i_{p}(1) \geq 2$ if $w_{P}(0)<2 / 3$.

Proof. Let the notation be as in (4.9). Since $C^{\sharp}$ is not smooth, ow $\left(C^{\sharp}\right)$ is not generated by ow 2 (2.7). Hence ow $\mathrm{ow}_{1} \mathrm{ow}_{2} \notin \mathrm{ow}\left(C^{\sharp}\right)$ by ow $\left(C^{\sharp}\right)=\mathbb{Z}_{+} \mathrm{ow}_{1}+$ $\mathbb{Z}_{+}$ow $_{2}$. Hence $U\left(\mathrm{ow}_{2}-\mathrm{ow}_{1}\right) \geq 1$ (2.8). By (4.9(ii)), we have

$$
\begin{aligned}
w_{P}^{*}(1) & =U\left(s a_{2} \mathrm{ow}_{1}+\mathrm{ow}_{2}\right)+U\left(\mathrm{ow}_{2}-\mathrm{ow}_{1}\right)-2 \\
& =U\left(s a_{2} \mathrm{ow}_{1}+\mathrm{ow}_{2}\right)-1 .
\end{aligned}
$$

By definition of $U(2.8)$, we have $U\left(s a_{2} \mathrm{ow}_{1}+\mathrm{ow}_{2}\right) \geq U\left(s a_{2} \mathrm{ow}_{1}\right)=i_{P}(1)$. Whence $w_{P}^{*}(1) \geq i_{P}(1)-1$ and (i) is proved. Assume now that $i_{P}(1)=$ $U\left(s a_{1}\right.$ ow $) \leq 1$ and $w_{P}(0)=\left(\bar{m}-a_{2}\right) / \bar{m}<2 / 3$ (4.9) to prove (ii). Then $(\bar{m}, 0)-s a_{1} \cdot \mathrm{ow}_{2} \in \mathrm{ow}\left(C^{\sharp}\right)$ and $3 a_{2}>\bar{m}$. Comparing the order part, we see $s=2, a_{1}=1$, and $(\bar{m}, 0)-2 \mathrm{ow}_{2}=\alpha \mathrm{ow}_{1}$ for some $\alpha \in \mathbb{Z}_{+}$. Thus $\bar{m}=2 a_{2}+\alpha$ and $2 a_{2}+\alpha$ wt $x_{1} \equiv 0(2 \bar{m})$. Hence wt $x_{1} \not \equiv 1(2 \bar{m})$, and wt $x_{1} \equiv 1+\bar{m}(2 \bar{m})$. Since $\left(\right.$ wt $\left.x_{1}, 2 \bar{m}\right)=1, \bar{m}$ must be even, whence $\alpha=\bar{m}=2 a_{2}$ is even. Then $\bar{m}=2 a_{2}+\alpha \equiv 2 a_{2}+\alpha$ wt $x_{1} \equiv 0(2 \bar{m})$, which is a contradiction.

(5.7) Proposition. Let $P$ be a type $\left(\mathrm{IC}^{\vee}\right)$ point. Then $i_{P}(1)=2, w_{P}^{*}(1)=2$, and $w_{P}(0) \geq 3 / 4$.

Proof. Since ow $\left(C^{\sharp}\right)=\mathbb{Z}_{+}(1,1)+\mathbb{Z}_{+}(\bar{m}-1,-1)$, we can easily see

for integers $x \in[1,2 \bar{m}]$. Thus

$$
U(x)= \begin{cases}1 & \text { if } x=1, \\ 2 & \text { if } 2 \leq x \leq 2 \bar{m}\end{cases}
$$

$$
\begin{gathered}
i_{P}(1)=U(2 \bar{m}-2)=2, \\
w_{P}(0)=R(\bar{m}+1) / \bar{m}=U(\bar{m}+1)-(\bar{m}+1) / \bar{m}=(\bar{m}-1) / \bar{m} \geq 3 / 4,
\end{gathered}
$$

and

$$
\begin{aligned}
w_{P}^{*}(1) & =U(2 \bar{m}-2+\bar{m}+1)-U(\bar{m}+1)+U(2 \bar{m}+2)-2 U(\bar{m}+1) \\
& =2+U(\bar{m}-1)-U(\bar{m}+1)+2+U(2)-2 U(\bar{m}+1) \\
& =2
\end{aligned}
$$

by $\bar{m} \geq 4(4.9(\mathrm{ii}))$.

6. Possible singularities on AN EXTREMal NBD $X \supset C \simeq \mathbb{P}^{1}$

The main purpose of this section is to limit the possible combination of singularities of types (IA)-(III), $\left(\mathrm{IA}^{\vee}\right)-\left(\mathrm{II}^{\vee}\right)$ using invariants $i_{P}(d), w_{P}(0)$, and $w_{P}^{*}(d)$.

The results are summarized in (6.7). Easy cases (6.7.1)-(6.7.3) will be treated in $\S 7$ and the delicate case (6.7.4) will be treated in $\S 9$.

We consider imprimitive points first, since they are much easier to treat. 
(6.1) Theorem. Let $X \supset C \simeq \mathbb{P}^{1}$ be an extremal nbd with an imprimitive point $P$. Then

(i) $P$ is of type $\left(\mathrm{IA}^{\vee}\right)$ or $\left(\mathrm{II}^{\vee}\right)$ (i.e. not of type $\left(\mathrm{IC}^{\vee}\right)$ ),

(ii) $X \supset C$ has at most one more singular point, and

(iii) if $w_{P}(0)<2 / 3$ or $\operatorname{siz}_{P} \geq 2$ (in particular if $P$ is of type $\left(\mathrm{II}^{\vee}\right)$ ), then $P$ is the only singular point of $X$ on $C$.

Proof. By (1.13), $X \supset C$ has no other imprimitive points. Since $L$-deformation (4.7) at a type $\left(\mathrm{IA}^{\vee}\right)$ or $\left(\mathrm{II}^{\vee}\right)$ point $P$ produces an extremal nbd $Y \supset D$ with an ordinary type $\left(\mathrm{IA}^{\vee}\right)$ point $Q$ such that $\operatorname{siz}_{Q}=\operatorname{siz}_{P}, w_{Q}(0)=w_{P}(0)$, and $Y \supset D$ is isomorphic to $X \supset C$ outside small nbds of $Q$ and $P$, we may replace $X \supset C$ with an $L$-deformation at $P$. Also by replacing $X \supset C$ with an $L$-deformation at other points, we may assume that $X \supset C$ has only ordinary points $(4.7)$, hence has only points of type (IA), (IA $\left.{ }^{\vee}\right),(I C),\left(I^{\vee}\right)$, (III).

(i) Assume that $X \supset C$ has a type $\left(\mathrm{IC}^{\vee}\right)$ point $P$. Then by $w_{P}^{*}(1)=2$ (5.7), $X \supset C$ has another singular point $Q$ such that $w_{Q}^{*}(1) \leq-1$ (2.3.3). By $i_{P}(1)=2(5.7)$, we have $i_{Q}(1)=1$ by (2.3.3) and (2.15). Thus $Q$ is not of type (IC) by $(5.5(\mathrm{i}))$, and not of type (III) by (4.9(ii)), hence of type (IA). By $w_{Q}^{*}(1) \leq-1$, we have $w_{Q}(0) \geq 1 / 2$ (5.2(iii)), which contradicts $w_{P}(0)+w_{Q}(0)<1 \quad(2.3 .3)$ because $w_{P}(0) \geq 1 / 2(5.7)$. Hence $P$ cannot be a type $\left(\mathrm{IC}^{\vee}\right)$ point.

(ii) Assume that $X \supset C$ has two more singular points $Q$ and $R$. Then by (2.3.3) and (2.15), we have $i_{P}(1)=i_{Q}(1)=i_{R}(1)=1$ and no other singular points. Hence $Q$ and $R$ are not of type (IC) by (5.5(i)). By $w_{P}(0) \geq 1 / 2$ (4.2), we see $w_{Q}(0), w_{R}(0)<1 / 2$ by (2.3.3). Then we see $w_{Q}^{*}(1)=w_{R}^{*}(1)=1$, by (4.9(i)) for type (III) points, and by (5.1(ii)) with $d=1$ for type (IA) points. We also have $w_{P}^{*}(1) \geq i_{P}(1)-1=0$ by $(5.6(\mathrm{i}))$. Hence $w_{P}^{*}(1)+w_{Q}^{*}(1)+w_{R}^{*}(1) \geq$ 2 , which contradicts $(2.3 .3)$.

(iii) Assume that $w_{P}(0)<2 / 3$ or $\operatorname{siz}_{P} \geq 2$ and that $X \supset C$ has another singular point $Q$. Then $i_{P}(1) \geq 2$ by $(5.6(\mathrm{ii}))$ or $(4.10)$, and $w_{P}^{*}(1) \geq i_{P}(1)-$ $1 \geq 1(5.6(\mathrm{i}))$. Thus by (2.3.3) and (2.15), one sees that $P$ and $Q$ are the only singular points, $i_{Q}(1)=1$ and $w_{Q}^{*}(1) \leq 0$. Thus $Q$ is a type (IA) point as in (i). Then by $w_{Q}(0)<1-w_{P}(0) \leq 1 / 2(4.2)$, we have $w_{Q}^{*}(1)=1$ by $(5.1(\mathrm{ii}))$ with $d=1$. This is a contradiction.

(6.2) Theorem. Let $X \supset C \simeq \mathbb{P}^{1}$ be an extremal nbd. Then

(i) $X$ has at most three singular points on $C$ and at least one of them has index $>1$, and

(ii) if $X$ has three singular points on $C$, then they consist of a type (III) point, a type (IA) point of index 2 , and a type (IA) point (say, $P$ ) of odd index ( say, $m \geq 3$ ) and size 1 such that $w_{P}(0)=(m-1) /(2 \cdot m)$. 
Proof. By (6.1), we may assume that $X \supset C$ is locally primitive. By (2.3.3), we have $\sum_{P} i_{P}(1) \leq 3$. Since $i_{P}(1) \geq 0$ for all $p \in C$ and $i_{P}(1) \geq 1$ for singular $P$ by (2.15), one sees that $X$ has at most three singular points on $C$. For (i), it is enough to derive a contradiction assuming that $X \supset C$ has only type (III) singular points. Applying $L$-smoothing (4.7) repeatedly at type (III) points of $X \supset C$, one obtains a locally primitive extremal nbd $Y \supset D \simeq \mathbb{P}^{1}$ such that $Y$ is smooth. By (2.3.2), one has $\operatorname{deg} \operatorname{gr}_{D}^{1} \mathscr{O}=1$ because $i_{P}(1)=0$ for smooth points $P$. Thus $\operatorname{deg} N_{D / Y}=-\operatorname{deg} \operatorname{gr}_{D}^{1} \mathscr{O}=-1$ and the space of embedded deformation of $D$ in $Y$ has dimension $\geq \chi\left(N_{D / Y}\right)=2-1=1$. This means that $D$ moves inside $Y$ while it is the only reduced compact subvariety of dimension $\geq 1$ in its small enough neighborhood. This is a contradiction and (i) is proved.

We will prove (ii). Let $P, Q, R$ be the singular points with indices $k, m$, $n$, respectively. As in the proof of $(\mathrm{i})$, one has $i_{P}(1)=i_{Q}(1)=i_{R}(1)=1$ and $\operatorname{deg} \operatorname{gr}_{C}^{1} \mathscr{O}=-2$. We note that $X \supset C$ has no points of type (IC) by $(5.5(\mathrm{i}))$. Thus $P, Q, R$ are of type (IA), (IB), (IIA), (IIB), or (III). We treat four cases (modulo permutation of $P, Q, R$ ).

(6.2.1) Case ordinary $P, Q, R$ of type (IA), (III), (III), respectively. We will derive a contradiction in this case. By $w_{P}^{*}(1)+w_{Q}^{*}(1)+w_{R}^{*}(1) \leq 1$ (2.3.3) and $w_{Q}^{*}(1)=w_{R}^{*}(1)=1$ (4.9(ii)), one has $w_{P}^{*}(1) \leq-1$. Thus by $(5.2(\mathrm{iii}))$, one has $w_{P}^{*}(d)=-\lceil d / 2\rceil+\delta_{d, k}$ for $d \in[1, k]$. Let $d \in[1, k]$ and let $r \in[0,1]$ be such that $r \equiv d(2)$. By $w_{Q}^{*}(d)=w_{R}^{*}(d)=\left[(d+1)^{2} / 4\right]$ (4.9(ii)), one has

$$
\begin{aligned}
1+d+\operatorname{deggr}{ }_{C}^{d} \omega & =d+1+\frac{1}{2}(d+1)(d-2)+\left\lceil\frac{d}{2}\right\rceil-\delta_{d, k}-2\left\lceil\frac{(d+1)^{2}}{4}\right\rceil \\
& =\frac{1}{2} d(d+1)+\frac{d+r}{2}-\delta_{d, k}-\frac{(d+1)^{2}-(1-r)}{2}=-\delta_{d, k},
\end{aligned}
$$

by (2.3.2). Thus we have

$$
\sum_{d=1}^{k}\left(1+d+\operatorname{deg}^{d} r_{C}^{d} \omega\right)=-1<0,
$$

which contradicts (2.3.3).

(6.2.2) Case ordinary $P, Q, R$, all of type (IA) with $w_{P}(0) \geq w_{Q}(0) \geq w_{R}(0)$. We will also derive a contradiction in this case. By $w_{P}(0)+w_{Q}(0)+w_{R}(0)<$ 1 (2.3.3), one has $w_{Q}(0), w_{R}(0)<1 / 2$. Hence by $(5.1(\mathrm{ii}))$ with $d=1$, one has $i_{Q}(2)=i_{R}(2)=0$. Hence by $\operatorname{deggr}_{C}^{1} \mathscr{O}=-2$ and (2.3.3), one has $\operatorname{deg} \operatorname{gr}_{C}^{2} \mathscr{O} \geq-3$ and whence $-6+i_{P}(2)+i_{Q}(2)+i_{R}(2) \geq-3$, i.e. $i_{P}(2) \geq 3$ by (2.3.2). Applying (5.2(i)) to $P$, one has $a_{2}=1$ under the notation of (5.2), whence $3 \leq i_{P}(2)=2+\delta_{2, k}$ by $\left(5.2(\right.$ iii) $)$. Thus $k=2$ and $\operatorname{deg}_{g_{C}}^{2} \mathscr{O}=-3$, whence $w_{P}(0)=1 / 2$ and $i_{P}(d)=(d+1)[d / 2]$ for $d \geq 2$ by (5.3). One 
has $w_{R}(0)<\left(1-w_{P}(0)\right) / 2=1 / 4$, and $i_{R}(3)=0$ follows from $(5.1(\mathrm{ii}))$ with $d=2$. From $\operatorname{deg}^{d} \operatorname{gr}_{C}^{d} \mathscr{O}=-d-1(d=1,2)$, one has $-4 \leq \operatorname{deg}^{3} \operatorname{gr}_{C}^{3} \mathscr{O}=$ $-3 \cdot 4+i_{P}(3)+i_{Q}(3)+i_{R}(3)=-8+i_{Q}(3)$ by (2.3.2) and (2.3.3). Hence $w_{Q}(0)<1-w_{P}(0)=1 / 2$ and $i_{Q}(3) \geq 4$, whence $m=3$ and $w_{Q}(0)=1 / 3$ by (5.1(ii)) with $d=2$. Thus $i_{Q}(d)=(d+1)[d / 3]$ for $d \geq 2$ by (5.3). From $w_{R}(0)<1-w_{P}(0)-w_{Q}(0)=1 / 6$, one has $i_{R}(4)=i_{R}(5)=0$. Thus by (2.3.2), one has

$$
\begin{aligned}
& \operatorname{deg} \operatorname{gr}_{C}^{4} \mathscr{O}=-4 \cdot 5+i_{P}(4)+i_{Q}(4)=-20+10+5=-5 \text {, } \\
& \operatorname{deg} \operatorname{gr}_{C}^{5} \mathscr{O}=-5 \cdot 6+i_{P}(5)+i_{Q}(5)=-30+12+6=-12 \text {, }
\end{aligned}
$$

which contradicts $\operatorname{deg}^{4}{ }_{C}^{4} \mathscr{O}+\operatorname{deg}_{C} \operatorname{gr}_{C}^{5} \mathscr{O} \geq-5-6$ (2.3.3). So this case does not occur.

(6.2.3) Case ordinary $P, Q, R$ of type (IA), (IA), (III), respectively, with $w_{P}(0) \geq w_{Q}(0)$. We will prove $k=2, m \equiv 1(2)$, and $w_{Q}(0)=(m-1) / 2 m$ in this case. By $w_{Q}(0) \leq w_{P}(0)$ and $w_{P}(0)+w_{Q}(0)<1$ (2.3.3), one has $w_{Q}(0)<1 / 2$ and hence $w_{Q}^{*}(1)=1$ by $(5.1(\mathrm{ii}))$ with $d=1$. By $w_{R}^{*}(1)=1$ $(4.9(\mathrm{ii}))$ and $w_{P}^{*}(1) \leq 1-w_{Q}^{*}(1)-w_{R}^{*}(1)=-1(2.3 .3)$, one has $w_{P}^{*}(1)=-1$, $w_{P}(0)=(k-1) / k, w_{P}^{*}(2)=-1+\delta_{2, k}$ by $(5.2(\mathrm{iii}))$. Hence $\operatorname{deg} \operatorname{gr}_{C}^{1} \omega=$ $-1-w_{P}^{*}(1)-w_{Q}^{*}(1)-w_{R}^{*}(1)=-2$ (2.3.2) and $\operatorname{deg} \operatorname{gr}_{C}^{2} \omega \geq-3$ (2.3.3), whence $-3 \leq \operatorname{deg} \operatorname{gr}_{C}^{2} \omega=-w_{P}^{*}(2)-w_{Q}^{*}(2)-w_{R}^{*}(2)$ (2.3.2). By $w_{R}^{*}(2)=2$ (4.9(ii)), one has $w_{Q}^{*}(2) \leq 2-\delta_{2, k}$. We claim $k=2$. Indeed if $k \geq 3$, then $w_{Q}(0)<1-w_{P}(0)=1 / k \leq 1 / 3$ and one sees $w_{Q}^{*}(2)=3$ by $(5.1(\mathrm{ii}))$ with $d=2$, which contradicts $w_{Q}^{*}(2) \leq 2-\delta_{2, k}$. Thus $k=2$ is proved, and hence $w_{Q}^{*}(2) \leq 1$ and $w_{Q}(0)<1 / 2$. By $(5.1(\mathrm{ii}))$ with $d=2$, one sees $w_{Q}(0)=(m-1) / 2 m$ and $m \equiv 1$ (2) .

(6.2.4) General case. We claim that $X \supset C$ has no points of type (IB) or (IIB) (resp. (IIA)). Indeed if otherwise, we may apply $L$-deformation (4.7) (repeatedly) at such points to produce a locally primitive extremal nbd $Y \supset D$ with only ordinary singular points which has at least four singular points (resp. one point with index 4 and at least two more singular points), which contradicts (i) (resp. (6.2.1)-(6.2.3)). Thus the claim is proved and $X \supset C$ has only points of type (IA) or (III) with $i(1)=1$. When we apply $L$-deformation (4.7) repeatedly at these points to produce a locally primitive extremal nbd with only ordinary singular points, each singular point deforms to exactly one singular point of the same type with the same index, size, and $w(0)$. Hence we are done by $(6.2 .1)-(6.2 .3)$.

(6.3) Theorem. Let $X \supset C \simeq \mathbb{P}^{1}$ be an extremal nbd. Then $X \supset C$ has no type (IB) points.

Proof. We will derive a contradiction assuming that $X \supset C$ contains a type (IB) point $P$. We may apply $L$-deformation (4.7) repeatedly to $X \supset C$, and obtain 
an extremal nbd $Y \supset D \simeq \mathbb{P}^{1}$ with only ordinary singular points which has at least two points $P$ and $Q$ of type (IA) with the same index $m(>1)$ such that $w_{P}(0)=w_{Q}(0)$. By (6.1) and (6.2), $P$ and $Q$ are the only singular points of $Y$ on $D$. By $w_{P}(0)=w_{Q}(0)<1 / 2(2.3 .3)$, one has $w_{P}^{*}(1)=w_{Q}^{*}(1)=1$ by (5.1(ii)) with $d=1$, which contradicts (2.3.3). Hence $X \supset C$ has no type (IB) points.

(6.4) Theorem. Let $X \supset C \simeq \mathbb{P}^{1}$ be an extremal nbd. If $X \supset C$ has a point $P$ of type (IIB), then $P$ is the only singular point of $X$ on $C$.

Proof. If $X \supset C$ has singular points other than $P$, then one may apply $L$-deformation (4.7) at type (IIB) point $P$ to $X \supset C$ to get an extremal nbd $Y \supset D \simeq \mathbb{P}^{1}$ with at least three singular points, two of which have indices 2 and 4. This contradicts (6.2).

(6.5) Theorem. Let $X \supset C \simeq \mathbb{P}^{1}$ be an extremal nbd. Assume that $X \supset C$ has a type (IC) point $P$ with index $m$. Then $P$ is the only singular point of $X$ on $C$, and one has $i_{P}(1)=2$ and $w_{P}(0)=(m-1) / m$.

Proof. If $X \supset C$ has an imprimitive point $Q(\neq P)$, then we may pass to the splitting cover (1.12) $Y \supset D$ because the point over $Q$ has index $>1$ (1.16) and $Y$ is locally primitive along an arbitrary irreducible component of $D$ (1.13). Hence we may assume that $X \supset C$ is locally primitive.

Using $L$-deformation (4.7), one may assume that $X \supset C$ has only ordinary singularities. We note $i_{P}(1) \geq 2(5.5(\mathrm{i}))$. We treat three cases.

(6.5.1) Case $i_{P}(1) \geq 3$. We will derive a contradiction in this case. One has $i_{P}(1)=3$ and $P$ is the only singular point of $X$ on $C$ by (2.3.3) and (2.15), whence $w_{P}^{*}(1) \leq 1$ by (2.3.3). This is a contradiction, because $(5.5(\mathrm{i}))$ and (5.5(ii)) show $w_{P}^{*}(1) \geq 3-1=2$.

(6.5.2) Case $i_{P}(1)=2$ and $w_{P}(0) \neq(m-1) / m$. We will derive a contradiction in this case. By $(5.5(\mathrm{ii}))$ and $(5.5(\mathrm{iii}))$, one has $w_{P}^{*}(1)=2$ and $i_{P}(2)=0$. Hence by (2.3.3) and $i_{P}(1)=2$, one sees that there exists exactly one more singular point $Q$ of $X$ (say, with index $n$ ) on $C, i_{Q}(1)=1, \operatorname{deg} \operatorname{gr}_{C}^{1} \mathscr{O}=-2$ and $w_{Q}^{*}(1) \leq-1$. We see that $Q$ is not of type (IC) by (5.5(i)) and hence $Q$ is of type (IA) by (4.9(ii)). By (5.2(iii)), one has $w_{Q}(0)=(n-1) / n, i_{Q}(2)=2+\delta_{2, n}$, and $i_{Q}(3)=4+\delta_{3, n}$. By $\operatorname{deg} \operatorname{gr}_{C}^{1} \mathscr{O}=-2$, one sees

$$
-3 \leq \operatorname{deg}_{C}^{2} \mathscr{O}=-6+i_{P}(2)+i_{Q}(2)=-4+\delta_{2, n}
$$

by (2.3.2) and (2.3.3). Thus $n=2, \operatorname{deg}_{C}^{2} \mathscr{O}=-3$, and $w_{P}(0)<1-w_{Q}(0)=$ $1 / 2$. By $(5.5(\mathrm{iv}))$ with $d=2$, one has $i_{P}(3)=0$, and hence $\operatorname{deg}_{C}^{3} \mathscr{O}=$ $-12+i_{P}(3)+i_{Q}(3)=-8$ by (2.3.2). This contradicts $\operatorname{deggr}_{C}^{3} \mathscr{O} \geq-4$ (2.3.3).

(6.5.3) Case $i_{P}(1)=2$ and $w_{P}(0)=(m-1) / m$. We will derive a contradiction assuming that $X$ has another singular point $Q$ on $C(Q$ is unique by (2.3.3) 
and (2.15)). Since $P$ is of type (IC), one has $m \geq 5$, whence $w_{Q}(0)<1-$ $w_{P}(0) \leq 1 / 5$ (2.3.3). Since $i_{Q}(1)=1$ by (2.3.3), $Q$ is of type (IA) or (III). Let $Q$ be of type (IA) (resp. (III)). Then by $w_{Q}(0) \leq 1 / 5$ and (5.1(ii)) (resp. by $(4.9(\mathrm{ii})))$ with $d \in[1,3]$, one has $w_{Q}^{*}(d)=d(d+1) / 2\left(\operatorname{resp} .\left[(d+1)^{2} / 4\right]\right)$ for $d \in[1,3]$. By $(5.5(\mathrm{ii}))$, one has $w_{P}^{*}(1)=1-\delta_{m .5}$. Thus by $(2.3 .3)$, one has $1 \geq w_{P}^{*}(1)+w_{Q}^{*}(1)=2-\delta_{m, 5}$, whence $m=5$. Hence by $(5.5(\mathrm{v}))$ and $(2.3 .2)$, one has

$$
\begin{aligned}
\sum_{d=1}^{3}\left(1+d+\operatorname{deg} \operatorname{gr}_{C}^{d} \omega\right) & =\sum_{d=1}^{3} \frac{d(d+1)}{2}-4-\sum_{d=1}^{3} \frac{d(d+1)}{2}=-4<0 \\
& \left(\text { resp. } \sum_{d=1}^{3} \frac{d(d+1)}{2}-4-\sum_{d=1}^{3}\left[\frac{(d+1)^{2}}{4}\right]=-1<0\right) .
\end{aligned}
$$

This contradicts (2.3.3), and we are done.

(6.6) Theorem. Let $X \supset C \simeq \mathbb{P}^{1}$ be an extremal nbd. Assume that $X \supset C$ has a type (IA) point $P$ of size $>1$. Then $P$ is the only singular point of $X$ on $C, \operatorname{siz}_{P}=2$, and $w_{P}(0)>1 / 2$.

Proof. As in the proof of (6.5), we may assume that $X \supset C$ is locally primitive. By using $L$-deformation (4.7), one may assume that $X \supset C$ has only ordinary singular points of type (IA) or (III) (6.5). Then $i_{P}(1)=\operatorname{siz}_{P}>1$ (4.10). Let $m$ be the index of $P$, and $a_{1}, \ldots, a_{4}$ as in the definition of type (IA) in (4.2). We treat three cases.

(6.6.1) Case $i_{P}(1) \geq 3$. We will derive a contradiction in this case. By $i_{P}(1) \geq$ 3 , one has $\operatorname{ord}\left(C^{\sharp}\right) \neq \mathbb{Z}_{+}$and $a_{2}>1$, whence $w_{P}^{*}(1) \geq 2$ by (5.2(ii)). Thus $P$ is the only singular point of $X \supset C$ by $\sum_{Q \neq P} i_{Q}(1)+3 \leq 3$ (2.3.3) and (2.15), while $X \supset C$ must have some other singular point by $\sum_{Q \neq P} w_{Q}^{*}(1)+2 \leq 1$ (2.3.3). This is a contradiction.

(6.6.2) Case $i_{P}(1)=2$ and $w_{P}(0) \geq 1 / 2$. We will first derive a contradiction assuming that $X \supset C$ has another singular point $Q$. By $\sum_{R \neq P . Q} i_{R}(1)+2+$ $i_{Q}(1) \leq 3$ (2.3.3) and (2.15), $P$ and $Q$ are the only singular points of $X \supset C$ and $i_{Q}(1)=1$. One has $w_{Q}(0)<1-w_{P}(0) \leq 1 / 2$ by (2.3.3). Then one sees that $w_{Q}^{*}(1)=1$ by $(5.1(\mathrm{ii}))$ if $Q$ is of type (IA), or by (4.9(ii)) if $Q$ is of type (III). Since $i_{P}(1)=2$, one has $w_{P}^{*}(1) \geq i_{P}(1)-1=1$ by $(5.2(\mathrm{i}))$, as in (6.6.1). Thus we have $w_{P}^{*}(1)+w_{Q}^{*}(1) \geq 2$, which contradicts $(2.3 .3)$. Hence $P$ is the only singular point in this case. We can see $w_{P}(0)=\left(m-a_{2}\right) / m \neq 1 / 2$ (4.9(i)). Indeed if otherwise, one sees $m=2 a_{2}$ and $a_{2}=1$ by $\left(a_{2}, m\right)=1$, whence $i_{P}(1)=1(4.9(\mathrm{ii}))$, which contradicts the hypothesis (6.6.2). Hence $w_{P}(0)>1 / 2$, which was to be proved in this case.

(6.6.3) Case $i_{P}(1)=2$ and $w_{P}(0)<1 / 2$. In this case, we will also derive a contradiction. By $(5.4)$, we have $a_{1}=2$ and $w_{P}^{*}(1) \geq 2$. Hence by 
$\sum_{Q \neq P} w_{Q}^{*}(1)+2 \leq 1$ (2.3.3), $X \supset C$ has another singular point $Q$ (say, of index $n)$ such that $w_{Q}^{*}(1) \leq-1$. By $\sum_{Q \neq P, Q} i_{R}(1)+2+i_{Q}(1) \leq 3$ (2.3.3) and (2.15), we see that $X \supset C$ has no other singular points, $i_{Q}(1)=1, \operatorname{deggr}_{C}^{1} \mathscr{O}=-2$. Now we claim

$$
n=2, \quad a_{2}=(m+1) / 2, \quad w_{P}^{*}(2)=3, \quad \operatorname{deggr}_{C}^{d} \omega=-d-1 \quad(d=1,2) .
$$

Since $w_{Q}^{*}(1) \leq-1, Q$ is a type (IA) point by $(4.9(\mathrm{ii}))$, whence

$$
w_{Q}(0)=(n-1) / n, \quad w_{Q}^{*}(1)=-1, \quad w_{Q}^{*}(2)=-1+\delta_{2, n}
$$

by $(5.2(\mathrm{iii}))$. By $w_{P}^{*}(1) \geq 2(5.4(\mathrm{ii}))$ and $w_{P}^{*}(1)+w_{Q}^{*}(1) \leq 1$ (2.3.3), one has $w_{P}^{*}(1)=2$ and $\operatorname{deggr}_{C}^{1} \omega=-2$ (2.3.3). Hence $\operatorname{deg} \operatorname{gr}_{C}^{2} \omega \geq-3$ by (2.3.3). If $n \geq 3$, then $w_{P}(0)<1-w_{Q}(0)=1 / n \leq 1 / 3 \quad(<2 / 5)$, whence $w_{P}^{*}(2) \geq 5$ (5.4(ii)) and we have a contradiction $-3 \leq-w_{P}^{*}(2)-w_{Q}^{*}(2) \leq-5+1-\delta_{2, n} \leq$ -4 from (2.3.2). Hence $n=2$ and $w_{Q}^{*}(2)=0$, and (2.3.2) implies $-3 \leq$ $\operatorname{deg} \operatorname{gr}_{C}^{2} \omega=-w_{P}^{*}(2)$, i.e. $w_{P}^{*}(2) \leq 3$. Thus by $(5.4(\mathrm{ii}))$, one sees $w_{P}^{*}(2)=3$, $a_{2}=(m+1) / 2$, and $\operatorname{deggr}_{C}^{2} \omega=-3$. This proves our claim (6.6.4).

By (2.3.4), one has $\operatorname{gr}_{C}^{1} \mathscr{O} \simeq \mathscr{O}(-1)^{\oplus 2}$ and $\operatorname{gr}_{C}^{d} \omega \simeq \mathscr{O}(-1)^{\oplus(d+1)}$ for $d \in$ [0,2]. Applying (4.8) to $X \supset C \ni Q$ (with $b_{1}=b_{2}=k=1$ and index 2), one sees that

$$
\beta_{2}: S^{2}\left(\mathrm{gr}_{C}^{1} \mathscr{O}\right) \otimes \mathrm{gr}_{C}^{0} \omega \hookrightarrow \mathrm{gr}_{C}^{2} \omega
$$

(cf. (2.2)) is described at $Q$ (by notation of (4.8)) as

$$
\left(\beta_{2}\right)_{Q}: \sum_{i+j=2} \mathscr{O} t^{3} v_{1}{ }^{i} v_{2}^{j} \Omega \hookrightarrow \sum_{i+j=2} \mathscr{O} t v_{1}^{i} v_{2}^{j} \Omega
$$

where $t^{2}$ is the uniformizing parameter of $\mathscr{O}_{C, Q}$. Hence it induces an injection

$$
\gamma_{2}: S^{2}\left(\mathrm{gr}_{C}^{1} \mathscr{O}\right) \otimes \mathrm{gr}_{C}^{0} \omega \otimes \mathscr{O}(Q) \hookrightarrow \mathrm{gr}_{C}^{2} \omega
$$

which is an isomorphism at $Q$ and hence an isomorphism outside $P$. We will study $\gamma_{2}$ near $P$ to derive a contradiction. Let $(x)$ and $t$ be normalized $\ell$-coordinates at $P$, and we will use $\left(X^{\sharp}, P^{\sharp}\right),\left(C^{\sharp}, P^{\sharp}\right)$, and wt $\left(=C^{\sharp}\right.$-wt) in the sense of $\S 2$. From now on we use $a$ instead of $a_{2}=(m+1) / 2$ for simplicity of notation. We note $a, m \equiv 1$ (2) by (5.4(i)) and $a \geq 3$. One has $\mathscr{O}_{C^{\sharp}, P^{\sharp}} \simeq \mathbb{C}\left\{t^{2}, t^{a}\right\}(2.7)$ and $\operatorname{gr}_{C^{\sharp}}^{1} \mathscr{O}$ (resp. $\left.\operatorname{gr}_{C^{\sharp}}^{0} \omega\right)$ has $\boldsymbol{\mu}_{m}$-semi-invariant free $\mathscr{O}_{C^{\sharp}, P^{\sharp}}$-basis $A$ and $B$ (resp. $C$ ) near $P^{\sharp}$ so that wt $A \equiv 2 a \equiv 1$, wt $B \equiv a_{3} \equiv-2 \quad(m)$ (resp. wt $C \equiv a(m)$ by (2.10)). This is because $\left(C^{\sharp}, P^{\sharp}\right)$ is a complete intersection $F_{2}=F_{3}=0$ in $\left(X^{\sharp}, P^{\sharp}\right)$, where wt $F_{2} \equiv 2 a$, wt $F_{3} \equiv a_{3}(m)$ (4.5.1). Hence $\operatorname{gr}_{C}^{1} \mathscr{O}=\mathscr{O}_{C}\left(t^{m-1} A\right) \oplus \mathscr{O}_{C}\left(t^{2} B\right)$ near $P$ by (4.8), where $t^{m-1}=\left(t^{2}\right)^{a-1}$ and $t^{2} \in \mathscr{O}_{C^{\sharp}, P^{\sharp}}$. We recall that $\mathscr{O}_{C, P}=\mathbb{C}\left\{t^{m}\right\}$ is the 
subring of $\mu_{m}$-invariants of $\mathscr{O}_{C^{\sharp}, P^{\sharp}}$. Since $\operatorname{gr}_{C}^{1} \mathscr{O} \simeq \mathscr{O}(-1)^{\oplus 2}$, one can choose a splitting into invertible sheaves

$$
\operatorname{gr}_{C}^{1} \mathscr{O}=L \oplus M
$$

so that $L \simeq \mathscr{O}(-1), M \simeq \mathscr{O}(-1), L \otimes \mathbb{C}(P)=\mathbb{C}(P)\left(t^{m-1} A\right)$, and $M \otimes \mathbb{C}(P)=$ $\mathbb{C}(P)\left(t^{2} B\right)$, where $\mathbb{C}(P)=\mathscr{O} / t \mathscr{O}$. Thus $L$ and $M$ have bases $\bar{L}$ and $\bar{M}$ near $P$ so that

$$
\bar{L}=t^{m-1} A+\lambda t^{m+2} B \text { and } \bar{M}=\mu t^{2 m-1} A+t^{2} B
$$

for some $\lambda, \mu \in \mathbb{C}\left\{t^{m}\right\}$. Since $t^{2 m-3}=t^{m} \cdot\left(t^{2}\right)^{(m-3) / 2} \in \mathscr{O}_{C^{\sharp}, P^{\sharp}}$, we will replace $B$ with $\mu t^{2 m-3} A+B$ so that one may set $\mu=0$ in (6.6.5). Let $\bar{N}$ be an $\mathscr{O}_{C^{-}}$ basis of $N=\mathrm{gr}_{C}^{0} \omega(\simeq \mathscr{O}(-1))$ near $P$ so that $\bar{N}=t^{m-a} C$ near $P$, where $t^{m-a}=\left(t^{2}\right)^{(m-a) / 2} \in \mathscr{O}_{C^{\sharp}, P^{\sharp}}$. We will show that $\gamma_{2}$ induces an isomorphism

(6.6.6) $\quad L^{2} \otimes N(2 P+Q) \oplus L \otimes M \otimes N(P+Q) \oplus M^{2} \otimes N(Q) \stackrel{\sim}{\longrightarrow} \mathrm{gr}_{C}^{2} \omega$

which will contradict $H^{1}\left(\mathrm{gr}_{C}^{2} \omega\right)=0$ because the source is isomorphic to $\mathscr{O} \oplus$ $\mathscr{O}(-1) \oplus \mathscr{O}(-2)$. By (4.8) and $a \geq 3$ above, one has

$$
\begin{aligned}
\operatorname{gr}_{C}^{2} \omega & =\bigoplus_{i, j \geq 0, i+j=2} \mathscr{O}_{C}{ }^{R((2 i+1) a-2 j)} A^{i} B^{j} C \\
& =\mathscr{O}_{C} t^{a-3} A^{2} C \oplus \mathscr{O}_{C} t^{a} A B C \oplus \mathscr{O}_{C} t^{a+3} B^{2} C
\end{aligned}
$$

near $P$ by direct calculation, or by

$$
\begin{aligned}
R((2 i+1) a-2 j) & =m \cdot U((2 i+1) a-2 j)-((2 i+1) a-2 j) \\
& =(i+1) m-(2 i+1) a+2 j \\
& =m-a-i+2 j=a-1-i+2 j
\end{aligned}
$$

where we used $U((2 i+1) a-2 j)=i+1$ by $(5.4(\mathrm{ii}))$ and $2 a=m+1$. Then

$$
\begin{aligned}
\bar{L}^{2} \bar{N} & =t^{2 m}\left(t^{m-a-2} A^{2} C+2 \lambda t^{a} A B C+\lambda^{2} t^{a+3} B^{2} C\right), \\
\bar{L} \cdot \bar{M} \cdot \bar{N} & =t^{m}\left(t^{a} A B C+\lambda t^{a+3} B^{2} C\right), \\
\bar{M}^{2} \bar{N} & =t^{a+3} B^{2} C,
\end{aligned}
$$

and $t^{-2 m} \bar{L}^{2} \bar{N}, t^{-m} \bar{L} \cdot \bar{M} \cdot \bar{N}, \bar{M}^{2} \bar{N}$ form an $\mathscr{O}_{C}$-basis for $\operatorname{gr}_{C}^{2} \omega$ near $P$. Since $\bar{L}^{i} \bar{M}^{j} \bar{N}$ is a basis at $P$ of components $L^{i} \otimes M^{j} \otimes N(Q)$ of $S^{2}\left(\mathrm{gr}_{C}^{1} \mathscr{O}\right) \otimes$ $\operatorname{gr}_{C}^{1} \omega \otimes \mathscr{O}(Q), \gamma_{2}$ induces homomorphisms $L^{i} \otimes M^{j} \otimes N(i P+Q) \rightarrow \mathrm{gr}_{C}^{2} \omega$ $\left(i, j \in \mathbb{Z}_{+}, i+j=2\right)$, which give splitting (6.6.6).

Thus we have a contradiction and case (6.6.3) does not occur. This proves (6.6). 
Summarizing the results in this section, we have

(6.7) Theorem. Let $X \supset C \simeq \mathbb{P}^{1}$ be an extremal nbd. Then we have three cases:

(i) Case $X$ has only one point $P$ with index $>1$. Then

(6.7.1) $P$ is of type (IA), (IIA), (IA $\left.{ }^{\vee}\right)$, or $\left(\mathrm{II}^{\vee}\right)$, and $X \supset C$ has at most one more singular point, which is a type (III) point, or

(6.7.2) $P$ is of type (IC) or (IIB), $X \supset C$ has no other singular points,

(ii) Case $X$ has $P$ of index $>1$ and $Q$ of index 2. Then

(6.7.3) $P, Q$ are of type (IA), $\operatorname{siz}_{P}=1, X \supset C$ has at most one more singular point, which is a type (III) point,

(iii) Case $X$ has points $P, Q$ of index $>2$. Then

(6.7.4) $P, Q$ are of type (IA), (IIA), or (IA $\left.{ }^{\vee}\right)$, and are of size 1 , and $X \supset C$ has no other singular points.

Proof. First, $X \supset C$ has a point with index $>1$ by (6.2), no type $\left(\mathrm{IC}^{\vee}\right)$ points by (6.1) and no type (IB) points by (6.3).

Case (i). If $P$ is of type (IA), (IIA), $\left(\mathrm{IA}^{\vee}\right)$, or $\left(\mathrm{II}^{\vee}\right)$, then $X \supset C$ has at most one more type (III) point by (6.2), whence we have (6.7.1). If $P$ is of type (IIB) or (IC), then $X \supset C$ has no other singular points by (6.4) or (6.5), whence we have (6.7.2).

In other cases, $X \supset C$ has no type (IC) (resp. (IIB), $\left(\right.$ II $\left.^{\vee}\right)$ ) points by $(6.5)$ (resp. (6.4), (6.1(iii)) and $\left.w_{P}(0)=1 / 2(4.9(\mathrm{i}))\right)$.

Case (ii). $X \supset C$ has at most one more type (III) point by (6.2), and $Q$ is of type (IA) because it is of index $2(4.2)$. Thus $w_{Q}(0)=1 / 2(4.9(\mathrm{i}))$ and $w_{P}(0)<1-w_{Q}(0)=1 / 2$ by (2.3.3). Hence $P$ cannot be of type (IIA) or $\left(\mathrm{IA}^{\vee}\right)$ and it is a type (IA) point with size 1 by $(6.6)$.

Case (iii). $X$ has no other singular points by (6.2). One sees that type (IIA) points have size 1 by definition and type $\left(\right.$ IA) $\left(\right.$ resp. $\left(\right.$ IA $\left.\left.^{\vee}\right)\right)$ points have size 1 by (6.6) (resp. (6.1)).

\section{EXISTENCE OF "GOOD” ANTI(BI)CANONICAL DIVISOR (EASY CASE).}

The main purpose of this section is to prove the existence of "good" members in $\left|-K_{X}\right|$ or $\left|-2 K_{X}\right|$ when extremal nbd $X \supset C \simeq \mathbb{P}^{1}$ has at most one point of index $>2$ (i.e. cases (6.7.1)-(6.7.3)). We start with quoting

(7.1) Theorem (Reid [R4, (6.3)]). Let $(X, P)$ be a 3-fold terminal singularity. Then general members of $\left|-K_{X}\right|$ passing through $P$ have only rational double points at $P$.

Following the same idea, one can show the following by a simple explicit calculation for $c A$ type points. We note that one can reduce (7.2) for arbitrary 3-fold terminal singularities to (7.1) by Kawamata's method in [Ka2, §8]. 
(7.2) Proposition. Let $(X, P)$ be a 3-fold cA-type terminal singularity of index $m>2$. Let $T$ be a general member of $\left|-2 K_{X}\right|$, and $(Y, Q) \rightarrow(X, P)$ the double cover with branch locus $T$. Then $(Y, Q)$ has only a canonical singularity.

(7.2.1) Remark. We note that a normal Gorenstein singularity $(Y, Q)$ is a canonical singularity iff it is a rational singularity [El2, Fl, SB].

Proof. Let $(x, y, z, u)$ (resp. $\phi)$ be an $\ell$-coordinate system (resp. $\ell$-equation) for $(X, P)$ such that wt $x+$ wt $y \equiv 0$, wt $u \equiv$ wt $\phi(m)$ (1a.5). Then the preimage of $T$ has a semi-invariant equation $\psi=\lambda z^{2}+\cdots$, and $(Y, Q)$ is the $\boldsymbol{\mu}_{m}$-quotient of $\left(Y^{\sharp}, Q^{\sharp}\right): \phi=v^{2}-\psi=0 \subset\left(\mathbb{C}^{5}, 0\right)$, where $v$ is an extra coordinate with wt $v \equiv$ wt $z(m)$ (cf. (2.10)). Since $(Y, Q)$ is an isolated singularity by Bertini's Theorem and Gorenstein by the adjunction formula, we only have to show that $\left(Y^{\sharp}, Q^{\sharp}\right)$ has only canonical singularities. We treat three cases.

(7.2.2) Case $m=4$ and $\mathrm{wt} \phi \equiv 2$ (4). We may assume that $\mathrm{wt}(x, y, z, u, \phi, v)$ $\equiv(3,1,1,2,2,1), \phi=x^{2}+z^{2}-f(y, u)$, where $f(y, u) \in\left(y^{2}, y^{2} u, u^{3}\right)$ by consideration of wt (1a.6). Since $\psi=\lambda x^{2}+u+\cdots \quad(\lambda \in \mathbb{C}), \quad\left(Y^{\sharp}, Q^{\sharp}\right)$ is an isolated hypersurface singularity $x^{2}+z^{2}-f\left(y, v^{2}-\lambda z^{2}-\cdots\right)=0$ in $x y z v$-space. Thus it is a $c A$ point, and hence is canonical (1a.2(ii)).

(7.2.3) Case $\phi=x y+f(z, u)$, wt $z^{2} \equiv$ wt $x(m)$. We note $f \in\left(z^{m}, u\right)$ by consideration of wt and $f(0, u) \neq 0$ by $u$-axis $\not \subset\left(X^{\sharp}, P^{\sharp}\right)$ (1a.6). Since wt $z^{2} \not \equiv 0$, wt $y$, wt $z$ by $m>2$, we may write $\psi=h \cdot\{x-g(y, z, u)\}$ for some $g$ and $h$ such that $h(0) \neq 0, g \in(y, z)^{2}$, and $z^{2}$ appears in $g$ by Weierstrass Preparation Theorem. Since $g$ and $h$ are unique by Weierstrass Preparation Theorem and since $\psi$ is semi-invariant, $g$ and $h$ are also semi-invariant. Then $h^{-1} v^{2}+g=x$, and replacing $v$ by $v h^{1 / 2}$, one may write $\left(Y^{\sharp}, Q^{\sharp}\right)$ as a hypersurface $F(y, z, u, v)=\left(v^{2}-g\right) y+f(z, u)=0$ in $y z u v$-space. Choosing a general $\psi$, one may assume that the plane curve

$$
c(F)(y, z, u) \equiv\left\{v^{2}-g_{2}(y, z, 0)\right\} y-f_{3}(z, 0)=0 \subset \mathbb{P}(y, z, v)
$$

has only ordinary double points, where (e.g.) $g_{n}$ denotes the degree $n$ part of $g$. Since $f(0, u) \neq 0$, it is enough to prove

\section{(7.2.3.1) Sublemma. Assume that a hypersurface singularity}

$$
(V, R): F(y, z, u, v) \equiv\left(v^{2}-g(y, z, u)\right) y+f(z, u)=0 \subset\left(\mathbb{C}^{4}, 0\right)
$$

such that $g \in(y, z)^{2}, f \in \mathbb{C}+\left(z^{3}, u\right)$, and $f(0, u) \neq 0$, has only canonical singularities outside $\{R\}$. If $c(F)=0 \subset \mathbb{P}(y, z, v)$ is a plane curve with only ordinary double points, then $V$ has only canonical singularities.

We prove this by induction on $k=$ ord $f(0, u)$. If $k=0,1$, then $F=0$ is smooth, and if $k=2$, then $R$ is a $c A$ or $c D$ point by its normality and hence canonical (1a.2(ii)). Assume $k \geq 3$. The exceptional divisor $D$ 
of the blow-up $\sigma: \bar{V} \rightarrow V$ of $V$ at $R$ lying in $\mathbb{P}(y, z, u, v)$ is defined by $c(F)(y, z, u)+\lambda u^{3}=0 \quad(\lambda \in \mathbb{C})$, and it is easy to see that $D$ has at most $A_{\infty}$-type singularity outside $(0: 0: 1: 0)$. Thus $\bar{V}$ is normal and has only rational singularities outside the point $\bar{R}$ corresponding to $(0: 0: 1: 0)$ above. Let $\bar{y}=y / u, \bar{z}=z / u, \bar{v}=v / u$ and $\bar{g}=g(\bar{y} u, \bar{z} u, u) u^{-2}, \bar{f}=f(z u, u) u^{-3}$, and $\bar{F}=\left(\bar{v}^{2}-\bar{g}\right)+\bar{f}$. Then $\bar{g} \in(\bar{y}, \bar{z})^{2}, \bar{f} \in \mathbb{C}+\left(\bar{z}^{3}, u\right)$, ord $\bar{f}(0, u)=k-3$, and $c(\bar{F})(\bar{y}, \bar{z}, \bar{v})=c(F)(\bar{y}, \bar{z}, \bar{v})$. Thus $\bar{V}$ has only rational singularities by the induction hypothesis, and $(V, R)$ is a canonical singularity because $K_{\bar{V}}=\sigma^{*} K_{V}$ as is easily seen, for example, by

$$
\sigma^{*} \operatorname{Res} \frac{d y \wedge d z \wedge d u \wedge d v}{F}=\operatorname{Res} \frac{d \bar{y} \wedge d \bar{z} \wedge d u \wedge d \bar{v}}{F}
$$

and the claim (7.2.3.1) is proved.

(7.2.4) Case $\phi=x y+f(z, u)$, wt $z^{2} \not \equiv$ wt $x$, wt $y(m)$. This case is quite similar to (7.2.3). It is easily reduced to the following

(7.2.4.1) Sublemma. Assume that a 3-fold $(V, R) \subset\left(\mathbb{C}^{5}, 0\right)$ defined by $F \equiv x y+f(z, u)=0$ and $G \equiv v^{2}+g(x, y, z, u)=0$ such that $g \in(x, y, z)^{2}$, $f \in \mathbb{C}+\left(z^{3}, u\right)$, and $f(0, u) \neq 0$, has only canonical singularities outside $\{R\}$. Then $V$ has only canonical singularities if we assume the following condition $(*)_{\lambda}$ for $\lambda=0$ and 1 .

Condition $(*)_{\lambda}: x y+\lambda u^{2}=0$ and $c(G)(x, y, z, v) \equiv v^{2}+g_{2}(x, y, z, 0)$ $=0$ define a surface $S_{\lambda}$ in $\mathbb{P}(x, y, z, u, v)$ with at most $A_{\infty}$-type singularities outside $(0: 0: 0: 1: 0)$.

The proof is similarly done by induction on $k=\operatorname{ord} f(0, u)$.

(7.2.4.2) It remains to see that $(*)_{0}$ and $(*)_{1}$ are satisfied by general $G$ (or $T \in|-2 K|)$. Since $S_{0}$ is the cone over the curve $Z \subset \mathbb{P}(x, y, z, v)$ defined by $x y=0$ and $c(G)=0,(*)_{0}$ is equivalent to the assertion that $Z$ has at most ordinary double points. Thus $(*)_{0}$ is certainly satisfied by general $G$ because it is satisfied by $G=v^{2}+\mu z^{2} \quad(\mu \in \mathbb{C}-\{0\})$.

On the other hand, $S_{1}$ is a double cover of the surface $W \subset \mathbb{P}(x, y, z, v)$ defined by $c(G)=0$ with branch locus $Z$. Thus one can see that $S_{1}$ has at most $A_{\infty}$-type singularities for general $G$ by the fact that $W$ has at most $A_{\infty}$-type singularities when $z^{2}$ appears in $G$, as follows. If $\xi \in S_{1}$ does not lie over a point in $Z$, then $S_{1} \rightarrow W$ is étale at $\xi$, whence $S_{1}$ has at most $A_{\infty}$-type singularity at $\xi$ because so does $W$ everywhere. If $\xi \in S_{1}$ lies over $\zeta \in Z$, then the Cartier divisor $(\{v=0\}, \xi)$ of $\left(S_{1}, \xi\right)$ is the ramification divisor and isomorphic to $(Z, \zeta)$. Since $(Z, \zeta)$ has at most an ordinary double point as above, $\left(S_{1}, \xi\right)$ has at most an $A_{\infty}$-type singular point. Thus the case (7.2.4) is finished, and (7.2) is proved.

(7.3) Theorem. Let $X \supset C \simeq \mathbb{P}^{1}$ be an extremal nbd. Assume that $X \supset C$ has at most one point of index $>2(\Leftrightarrow X \supset C$ is in one of (6.7.1), (6.7.2), 
(6.7.3)). We have three cases by (6.7):

(7.3.1) a point $P$ is of type (IA), (IIA), $\left(\mathrm{IA}^{\vee}\right)$, or $\left(\mathrm{II}^{\vee}\right)$, and no other point of index $>1 \quad(\Leftrightarrow(6.7 .1))$,

(7.3.2) a point $P$ is of type (IC) or (IIB), and no other singular point $(\Leftrightarrow$ (6.7.2)), and

(7.3.3) $P, Q$ are of type (IA), index $Q=2, \operatorname{siz}_{P}=1$, and no other points of index $>1 \quad(\Leftrightarrow(6.7 .3))$.

Then (i) in case (7.3.1), general members $(D, P)$ of $\left|-K_{(X, P)}\right|$ satisfy $D \cap C=\{P\}, D \in\left|-K_{X}\right|$ and that $D$ has only rational double points as singularities, and

(ii) in case (7.3.2) or (7.3.3), general members $(D, P)$ of $\left|-2 K_{(X, P)}\right|$ satisfy $D \cap C=\{P\}, D \in\left|-2 K_{X}\right|$ and that the double cover $Y$ of $X$ with branch locus $D$ has at most canonical singularities.

Proof. Let $(x)=\left(x_{1}, \ldots, x_{4}\right)$ and $t$ be normalized $\ell$-coordinates and $\phi$ an $\ell$-equation of $X \supset C$ at $P$ as in (4.2). Let $a_{1}, \ldots, a_{4}$ be as in (4.2). Then elements of $\mathscr{O}\left(-K_{X}\right)$ (resp. $\mathscr{O}\left(-2 K_{X}\right)$ ) at $P$ are of the form $f \Omega^{-1}$ (resp. $f \Omega^{-2}$ ), where $f$ is a semi-invariant in $\mathscr{O}_{X^{\sharp}, P^{\sharp}}$ such that wt $f \equiv$ wt $\Omega$ (resp. $2 \mathrm{wt} \Omega)$ and $\Omega=\operatorname{Res} d x_{1} \wedge d x_{2} \wedge d x_{3} \wedge d x_{4} / \phi$.

In case (7.3.1), $P$ is of type (IA), (IIA), $\left(\right.$ IA $\left.^{\vee}\right)$, or $\left(\right.$ II $\left.^{\vee}\right)$, whence wt $\Omega \equiv$ wt $x_{2}(m)$ by wt $x_{1} x_{3} \equiv 0$ and wt $x_{4} \equiv \mathrm{wt} \phi(4.2)$. Then a general $f$ with wt $f \equiv$ wt $\Omega(m)$ is $\lambda x_{2}+\cdots$ and $(D, P) \in\left|-K_{(X, P)}\right|$ defined by $f$ satisfies $(m D) \cdot C=a_{2} P$, and $D$ is a $\mathbb{Q}$-Cartier Weil divisor of $X$. One has $D \sim-K_{X}$ as elements of $\mathrm{Cl}(X, P)$ because wt $\Omega^{-1} \equiv$ wt $f^{-1}(\bmod m)$, whence $D+K_{X}$ is in Pic $X$ with $\left(D+K_{X} \cdot C\right)=0$ because $\left(K_{X} \cdot C\right)=-1+w_{P}(0)=-a_{2} / m$ (2.3.2). Thus $D \in\left|-K_{X}\right|(1.3(\mathrm{ii}))$, whence we are done by (7.1).

We note that, in the remaining cases, $X \supset C$ is locally primitive. In case (7.3.2), let $P$ be of type (IC) (resp. (IIB)). Then one has $a_{1}=2, a_{2}=m-2$, $a_{3}=m, a_{4} \equiv 1(m)$ (resp. $\left.\left(a_{1}, \ldots, a_{4}\right)=(3,2,5,5)\right)$ by $(4.9(\mathrm{ii})),(5.5(\mathrm{i}))$ and (6.5) (resp. definition). Hence wt $\Omega \equiv a_{4} \equiv 1(m)$, and a general $f$ with wt $f \equiv 2 \mathrm{wt} \Omega \equiv 2(m)$ is $\lambda x_{1}+\cdots$ by $a_{2}=2\left(\operatorname{resp} . \lambda x_{2}+\cdots\right.$ by $\left.a_{2}=2\right)$ with $\lambda \in \mathbb{C}^{*}$. Thus $(D, P) \in\left|-2 K_{(X, P)}\right|$ defined by $f$ satisfies $(m D) \cdot C=2 P$, and $D$ is a $\mathbb{Q}$-Cartier Weil divisor of $X$. One has $D \sim-2 K_{X}$ as elements of $\mathrm{Cl}(X, P)$, whence $D+2 K_{X}$ is in $\mathrm{Pic} X$ with $\left(D+2 K_{X} \cdot C\right)=0$ because $\left(K_{X} \cdot C\right)=-1+w_{P}(0)=-1 / m$ by (2.3.2) and (6.5) (resp. (4.9(ii))). Thus $D \in\left|-2 K_{X}\right|(1.3(\mathrm{ii}))$. We note that $P$ is of $c A$-type, by (1a.6) and $m \geq 5$ (part of definition) if $P$ is of type (IC), and by definition otherwise. Hence we are done by (7.2).

Let us consider (7.3.3). First we note that $w_{Q}(0)=1 / 2$ by $(4.9(\mathrm{i}))$ and index $Q=2$. Then $w_{P}(0)=\left(m-a_{2}\right) / m<1-w_{Q}(0)=1 / 2$ by $(2.3 .3)$ and $(4.9(\mathrm{i}))$, whence $2 a_{2}>m$. This means $m \geq 3$ since $m>a_{2}$ (4.2). On the other hand, one has $a_{1} a_{2} \leq m$ by $\operatorname{siz}_{p}=U\left(a_{1} a_{2}\right)=1$. Hence $a_{1}=1$. One has wt $\Omega \equiv a_{2}$ by $(2.10)$, and a general $f$ with wt $f \equiv 2$ wt $\Omega \equiv 2 a_{2}-m(m)$ is 
$\lambda x_{2}{ }^{2}+\mu x_{1}{ }^{2 a_{2}-m}+\cdots\left(\lambda, \mu \in \mathbb{C}^{*}\right)$. Thus $(D, P) \in\left|-2 K_{(X, P)}\right|$ defined by $f$ satisfies $(m D) \cdot C=\left(2 a_{2}-m\right) P$, and $D$ is a $\mathbb{Q}$-Cartier Weil divisor of $X$. We note that $D$ and $-2 K_{X}$ are Cartier divisors outside $\{P\}$ because index $Q=2$. Thus by $D \sim-2 K_{X}$ as elements of $\mathrm{Cl}(X, P)$, one sees $D \sim-2 K_{X}$ in Pic $X$ by

$$
\begin{aligned}
\left(-2 K_{X} \cdot C\right) & =-2\left(-1+w_{P}(0)+w_{Q}(0)\right) \\
& =-2\left(-1+\left(m-a_{2}\right) / m+1 / 2\right) \\
& =\left(2 a_{2}-m\right) / m .
\end{aligned}
$$

Hence $D \in\left|-2 K_{X}\right|$. We need to show that $P$ is of $c A$-type (1a.6). Indeed if $P$ is not of $c A$-type, then by $m \geq 3$ we see that $P$ is of $c D$-type and $m=3$ (1a.6). Thus by $(4.11)$, we have $w_{P}(0)=2 / 3$, which contradicts $w_{P}(0)<1 / 2$. Thus $P$ is of $c A$-type.

\section{8. $J$-FILTRATION AND LIFTING STRUCTURE}

We need to introduce several notions for the arguments in the next section. The main ingredients are rather simple commutative algebra lemmas (8.3) and (8.11), and other parts are formal arguments to obtain the main (8.12) from these.

We note that Reid's notion of width in $[R 1,(5.3)]$ is different from our width in (8.2).

(8.1) Let $X$ be an analytic normal 3-fold and $C \subset X$ a reduced curve such that no irreducible components of $C$ are contained in the singular locus of $X$.

(8.1.1) Lemma. Let $\mathscr{S}=\mathscr{S}_{0} \supset \mathscr{S}_{1} \supset \cdots \supset \mathscr{S}_{n}$ be a chain of coherent sheaves on $X$. Then we have equivalent conditions:

(a) $\mathscr{S}_{i}$ is $\mathscr{S}_{i-1}$-saturated for all $i \in[1, n]$,

(b) $\mathscr{S}_{i}$ is $\mathscr{S}_{0}$-saturated for all $i \in[1, n]$, and

(c) $\mathscr{S}_{i}$ is $\mathscr{S}_{j}$-saturated for all $i, j \in[0, n]$ with $j<i$.

Proof. First it is obvious that (c) $\Rightarrow(\mathrm{a}),(\mathrm{b})$. When $n=2,(\mathrm{a}) \Rightarrow(\mathrm{c})$ and (b) $\Rightarrow$ (c) amount to saying the following:

(*) When $\mathscr{S}_{1}$ is $\mathscr{S}_{0}$-saturated, $\mathscr{S}_{2}$ is $\mathscr{S}_{1}$-saturated iff $\mathscr{S}_{2}$ is $\mathscr{S}_{0}$-saturated.

Thus $n=2$ case is easily checked. Indeed for every closed point $P \in X$, we have

$$
\operatorname{Hom}\left(\mathbb{C}(P), \mathscr{S}_{0} / \mathscr{S}_{2}\right) \simeq \operatorname{Hom}\left(\mathbb{C}(P), \mathscr{S}_{1} / \mathscr{S}_{2}\right)
$$

from the exact sequence

$$
0 \rightarrow \mathscr{S}_{1} / \mathscr{S}_{2} \rightarrow \mathscr{S}_{0} / \mathscr{S}_{2} \rightarrow \mathscr{S}_{0} / \mathscr{S}_{1} \rightarrow 0
$$

and $\operatorname{Hom}\left(\mathbb{C}(P), \mathscr{S}_{0} / \mathscr{S}_{1}\right)=0$.

(a) $\Rightarrow$ (c) This is proved by induction on $j-i$ by virtue of the exact sequence

$$
0 \rightarrow \mathscr{S}_{j+1} / \mathscr{S}_{i} \rightarrow \mathscr{S}_{j} / \mathscr{S}_{i} \rightarrow \mathscr{S}_{j} / \mathscr{S}_{j+1} \rightarrow 0
$$

and the above $(*)$. 
(b) $\Rightarrow$ (c) This is proved by the above $(*)$ and the exact sequence

$$
0 \rightarrow \mathscr{S}_{j} / \mathscr{S}_{i} \rightarrow \mathscr{S}_{0} / \mathscr{S}_{i} \rightarrow \mathscr{S}_{0} / \mathscr{S}_{j} \rightarrow 0
$$

(8.2) Definition. Let $\mathscr{S}=\mathscr{S}_{0} \supset \mathscr{S}_{1} \supset \cdots \supset \mathscr{S}_{n}$ be a chain of coherent sheaves on $X$. We say that it is saturated if the equivalent conditions in (8.1.1) hold. We call the chain $\mathscr{S}_{0} \supset \operatorname{Sat}_{\mathscr{S}} \mathscr{S}_{1} \supset \cdots \supset \operatorname{Sat}_{\mathscr{S}} \mathscr{S}_{n}$ the saturation of the chain $\mathscr{S}_{0} \supset \cdots \supset \mathscr{S}_{n}$.

Let $J \subset \mathscr{O}_{X}$ be a sheaf of ideals. We say that $J$ is $C$-laminal (or laminal, if there is no danger of confusion) if $J$ has no embedded primary components, $I_{C}=\sqrt{J}$, and $I_{D}{ }^{(2)} \not \supset J$ for an arbitrary irreducible component $D$ of $C$ (cf. (2.2)). The width of $J$ is the smallest $d \in \mathbb{N}$ such that $J \supset I_{C}{ }^{d}$, and $J$ is said to be of pure width $d$ if the $D$-primary component of $J$ is a $D$-laminal ideal of width $d$ for each irreducible component $D$ of $C$.

Let $J$ be a $C$-laminal ideal of pure width $d$ and let $\mathscr{S}$ be a coherent $\mathscr{O}_{X}$-module. For $n \in \mathbb{Z}_{+}$, let $q=[n / d]$ and $r=n-d q$ (note $r \in[0, d-1]$ ). Let $F^{n}(\mathscr{S}, J)=\operatorname{Sat}_{\mathscr{S}}\left(J^{q} I_{C}{ }^{r}+J^{q+1}\right) \mathscr{S}, \operatorname{gr}^{n}(\mathscr{S}, J)=F^{n}(\mathscr{S}, J) / F^{n+1}(\mathscr{S}, J)$. One easily sees that

(8.2.1) Lemma. $F^{n}\left(\mathscr{O}_{X}, J\right) \cdot F^{n^{\prime}}(\mathscr{S}, J) \subset F^{n+n^{\prime}}(\mathscr{S}, J)$.

Proof. Let $q=[n / d], r=n-d q$, and $q^{\prime}=\left[n^{\prime} / d\right], r^{\prime}=n^{\prime}-d q^{\prime}$. Then $q^{\prime \prime}=\left[\left(n+n^{\prime}\right) / d\right]$ is given as $q^{\prime \prime}=q+q^{\prime}+\varepsilon$ for some $\varepsilon=0,1$, and $r^{\prime \prime}=$ $\left(n+n^{\prime}\right)-d q^{\prime \prime}$ is given as $r^{\prime \prime}=r+r^{\prime}-\varepsilon d$. It is enough to see

$$
\left(J^{q} I_{C}{ }^{r}+J^{q+1}\right) \cdot\left(J^{q^{\prime}} I_{C}{ }^{r^{\prime}}+J^{q^{\prime}+1}\right) \subset J^{q^{\prime \prime}} I_{C}^{r^{\prime \prime}}+J^{q^{\prime \prime}+1} .
$$

If $\varepsilon=0$, then this is obvious because $q^{\prime \prime}=q+q^{\prime}$ and $r^{\prime \prime}=r+r^{\prime}$. If $\varepsilon=1$, then we use $I_{C}{ }^{d} \subset J$. Indeed $J^{q} I_{C}{ }^{r} \cdot J^{q^{\prime}} I_{C}{ }^{r^{\prime}}=J^{q^{\prime \prime}-1} I_{C}{ }^{r^{\prime \prime}+d} \subset J^{q^{\prime \prime}} I_{C}{ }^{r^{\prime \prime}}$, $J^{q} I_{C}{ }^{r} \cdot J^{q^{\prime}+1}=J^{q^{\prime \prime}} I_{C}{ }^{r} \subset J^{q^{\prime \prime}} I_{C}{ }^{r^{\prime \prime}}$ by $r^{\prime \prime}=r+r^{\prime}-d<r$, and the rest is similar.

(8.2.2) Then

$$
\mathscr{S}=F^{0}(\mathscr{S}, J) \supset \cdots \supset F^{n}(\mathscr{S}, J) \supset F^{n+1}(\mathscr{S}, J) \supset \cdots
$$

is called the $J$-filtration, and $\operatorname{gr}^{\cdot}(\mathscr{O}, J)=\bigoplus_{n \geq 0} \operatorname{gr}^{n}(\mathscr{O}, J)$ is a graded $\mathscr{O}_{C^{-}}$-algebra and $\operatorname{gr}^{\cdot}(\mathscr{S}, J)=\bigoplus_{n \geq 0} \operatorname{gr}^{n}(\mathscr{S}, J)$ is a graded $\operatorname{gr}^{\circ}(\mathscr{O}, J)$-module.

For $s \in \mathscr{S}$, the width of $s$ with respect to $J$ (denoted by width ${ }_{J} s$ ) is given by width $_{J} s=\inf \left\{i \in \mathbb{Z}_{+} \mid s \in F^{i}(\mathscr{S}, J)\right\}$. It is easy to see but worthwhile to note that $F^{1}\left(\mathscr{O}_{X}, J\right)=I_{C}, F^{d}\left(\mathscr{O}_{X}, J\right)=J, \operatorname{gr}^{d}(\mathscr{O}, J)=\operatorname{gr}_{C}^{0} J$, and in particular the usage of width $_{J} s$ is compatible with width of $J$.

For $n=q d+r$ as above, one has a natural homomorphism

$$
\gamma^{n}(\mathscr{S}, J): S^{q}\left(\operatorname{gr}^{d}(\mathscr{O}, J)\right) \otimes\left(\operatorname{gr}^{1}(\mathscr{O}, J)\right)^{\otimes r} \otimes \operatorname{gr}_{C}^{0} \mathscr{S} \rightarrow \operatorname{gr}^{n}(\mathscr{S}, J) .
$$


We construct a 0 -sequence

$$
E(J): 0 \rightarrow \operatorname{gr}^{1}(\mathscr{O}, J) \stackrel{\otimes d}{\longrightarrow} \stackrel{\Delta_{J}}{\longrightarrow} \operatorname{gr}_{C}^{0}(J) \stackrel{\alpha_{J}}{\longrightarrow} \operatorname{Ker}\left[\operatorname{gr}_{C}^{1} \mathscr{O} \stackrel{\beta_{J}}{\longrightarrow} \operatorname{gr}^{1}(\mathscr{O}, J)\right] \rightarrow 0,
$$

together with the remarks: If $d=1$, then $J=I_{C}, F^{n}(\mathscr{S}, J)=F^{n} \mathscr{S}$, $\gamma^{n}(\mathscr{S}, J)=\gamma_{C}^{n}(\mathscr{S})(\S 2)$, and we set $\alpha_{J}=0$ and $\beta_{J}=\Delta_{J}=\mathrm{id}$, and $E(J)$ is exact. If $d \geq 2$, then $\alpha_{J}, \beta_{J}, \gamma_{J}$ are naturally induced homomorphisms, $E(J)$ is a 0 -sequence by $I_{C}{ }^{d} \subset J$ and $I_{C}^{(2)}+J \subset F^{2}(\mathscr{O}, J)$, and we note that $\operatorname{Ker} \beta_{J}$ and $\operatorname{gr}^{1}(\mathscr{O}, J)$ are of rank 1 .

(8.3) Proposition. Under the notation and assumptions of (8.1), assume that $(u, v)=I_{C, P}\left(=I_{C} \otimes \mathscr{O}_{X, P}\right)$ and let $d \geq 1$. Then

(i) $J=\left(u^{d}, v\right)$ is a C-laminal ideal of pure width $d$ in a nbd of $P$, and one has the following for all $n \geq 0$ :

$$
F^{n}\left(\mathscr{O}_{X}, J\right)_{P}=\sum_{i, j \geq 0, i+d j \geq n}\left(u^{i} v^{j}\right),
$$

and

$$
\operatorname{gr}^{n}(\mathscr{O}, J)_{P}=\bigoplus_{i, j \geq 0, i+d j=n} \mathscr{O}_{C, P} u^{i} v^{j} .
$$

Thus $E(J)$ is exact and $\gamma^{n}\left(\mathscr{O}_{X}, J\right)$ is isomorphic at $P$, and

(ii) let $J$ be a $C$-laminal ideal of pure width $d$ in a nbd of $P \in C$. Then $\left(u^{d}, v\right)=J_{P}\left(=J \otimes \mathscr{O}_{X, P}\right)$ iff $v \in J_{P}$.

Proof. (i) Since $(C, P)$ is Cohen-Macaulay, so is $(X, P)$. Hence $(u, v):(X, P)$ $\rightarrow\left(\mathbb{C}^{2}, 0\right)$ is flat since it is equidimensional. Since $K_{n}=\sum_{i+d j \geq n}\left(u^{i} v^{j}\right) \mathbb{C}\{u, v\}$ contains some power of maximal ideal $(u, v)$, it is $(u, v)$-primary. Thus by flatness, $K_{n} \mathscr{O}_{X, P}$ has no embedded primes. Then following the definition, one easily sees $F^{n}\left(\mathscr{O}_{X}, J\right)_{P}=K_{n} \mathscr{O}_{X, P}$. It is clear that $K_{n} / K_{n+1}=\bigoplus_{i+d j=n} \mathbb{C} u^{i} v^{j}$, whence one sees the assertion on $\operatorname{gr}^{n}(\mathscr{O}, J)_{P}$ by flatness of $(X, P) \rightarrow\left(\mathbb{C}^{2}, 0\right)$, and $\gamma^{n}\left(\mathscr{O}_{X}, J\right)_{P}$ is an isomorphism for all $n \geq 0$. If $d \geq 2$, then one sees $\operatorname{gr}^{1}(\mathscr{O}, J)_{P}=\mathscr{O}_{C, P} u, \operatorname{gr}_{C}^{0}(J)=\mathscr{O}_{C, P} u^{d} \oplus \mathscr{O}_{C, P} v, \operatorname{gr}_{C}^{1} \mathscr{O}=\mathscr{O}_{C, P} u \oplus \mathscr{O}_{C, P} v$, and $\operatorname{Ker} \beta_{J}=\mathscr{O}_{C, P} v$, whence $E(J)$ is exact.

(ii) The only-if part is obvious. Assume that $v \in J_{P}$. By (i), $\left(u^{d}, v\right)$ has no embedded primes at $P$, and $\left(u^{d}, v\right) \subset J_{P}$ by the definition of width. Thus it is enough to prove $\left(u^{d}, v\right)_{Q}=J_{Q}$ for close enough points $Q \in C-\{P\}$. Let $Q$ be a point such that $X$ and $C$ are smooth at $Q$, whence $u$ and $v$ form a part of coordinates at $Q$. Then $J_{Q} /(v)_{Q}$ is a divisorial ideal of a smooth surface $(\{v=0\}, Q)$ and one easily sees $J_{Q} /(v)_{Q}=\{(u, v) /(v)\}_{Q}^{d}=\left(u^{d}, v\right)_{Q} /(v)_{Q}$, whence $J_{Q}=\left(u^{d}, v\right)_{Q}$.

(8.4) Definition-Corollary. Let the notation and assumptions be as in (8.1). Let $J$ be a C-laminal ideal of pure width $d$. We say that $J$ is a nested complete 
intersection (nested c.i., in short) at $P$ if $(u, v)=I_{C, P}$ and $\left(u^{d}, v\right)=J_{P}$ for some $u$ and $v$, and that $J$ is locally a nest c.i. on a subset if $J$ is a nested c. $i$. at each point of the set. Then

(i) let $P \in C$. Then $J$ is a nested c.i. at $P$ iff $(C, P)$ is a complete intersection in $(X, P), \operatorname{Im} \alpha_{J}=\operatorname{Ker} \beta_{J}$, and $\operatorname{gr}^{1}(\mathscr{O}, J)$ is a locally free $\mathscr{\sigma}_{C}$-module at $P$, and

(ii) $J$ is locally a nested c.i. on an open dense subset of $C$. In particular, $\operatorname{Ker} \beta_{J}$ is the $\mathrm{gr}_{C}^{1} \mathscr{O}$-saturation of $\operatorname{Im} \alpha_{J}$.

Proof. (i) If $d=1$ then this is obvious; if $d \geq 2$ then the only-if part is done in $(8.3(\mathrm{i}))$. If $(C, P)$ is a c.i. in $(X, P)$, then $\operatorname{gr}_{C}^{1} \mathscr{O} \simeq I_{C} / I_{C}{ }^{2}$ is locally free of rank 2 at $P$. From the other assumptions, $\operatorname{Im} \alpha_{J} \simeq \operatorname{Ker} \beta_{J}$ and $\operatorname{Im} \beta_{J}$ are invertible at $P$. Thus there exist $v \in J_{P}$ and $u \in I_{C, P}$ such that $\operatorname{Im} \alpha_{J}=\mathscr{O}_{C} v$ and $\operatorname{Im} \beta_{J}=\mathscr{O}_{C} u$ in a nbd of $P$. Then $(u, v)=I_{C, P}$ by Nakayama's lemma, and $(8.3(\mathrm{ii}))$ proves our claim.

(ii) Let $d \geq 2$. Since $\operatorname{gr}_{C}^{1} \mathscr{O}\left(\right.$ resp. $\left.\operatorname{Im} \alpha_{J}, \operatorname{Ker} \beta_{J}, \operatorname{gr}^{1}(\mathscr{O}, J)\right)$ is of rank 2 (resp. $1,1,1$ ) on each irreducible component of $C$, one sees that $E(J)$ is exact on an open dense subset.

(8.5) Corollary. Let the notation and assumptions be as in (8.1). Let $J$ be a C-laminal ideal of pure width $d$ and $\mathscr{S}$ a torsion-free $\mathscr{O}_{X}$-module which is locally free of rank $\rho$ on an open set of $X$ containing an open dense subset of C. Let $n \geq 0$. Then

(i) $\gamma^{n}(\mathscr{S}, J)$ is injective at smooth points of $C$, and

(ii) $\gamma^{n}(\mathscr{S}, J)$ is isomorphic on an open dense subset of $C$ consisting of points at which $\mathscr{S}$ is locally free and $J$ is a nested c.i. In particular, $\operatorname{gr}^{n}(\mathscr{S}, J)$ is pure of rank $\rho[n / d]+\rho$ (i.e. of the same rank $\rho[n / d]+\rho$ on each irreducible component of $C$ ).

Proof. The assertion (i) follows from (ii) since the source of $\gamma^{n}(\mathscr{S}, J)$ is free at smooth points. The first part in (ii) follows from $(8.3(\mathrm{i}))$ and $(8.4(\mathrm{ii}))$, and the second from the first.

(8.6) Definition-Corollary. Under the notation and assumptions of (8.5), assume that $d \geq 2$, and let $q=[n / d]$, and $r=n-q d$. Then $E(J)$ induces $a$ saturated filtration (or chain)

$$
\operatorname{gr}^{n}(\mathscr{S}, J)=\Phi^{0} \operatorname{gr}^{n}(\mathscr{S}, J) \supset \cdots \supset \Phi^{i} \operatorname{gr}^{n}(\mathscr{S}, J) \supset \cdots \supset \Phi^{q+1} \operatorname{gr}^{n}(\mathscr{S}, J)=0
$$

such that $\operatorname{gr}^{n, i}(\mathscr{S}, J)=\Phi^{i} \operatorname{gr}^{n}(\mathscr{S}, J) / \Phi^{i+1} \operatorname{gr}^{n}(\mathscr{S}, J)$ is a torsion-free $\mathscr{O}_{C}$-module of pure rank $\rho$ for $i \in[0, q]$ and there is a naturally induced map $(i \in[0, q])$

$$
\gamma^{n, i}(\mathscr{S}, J): \operatorname{gr}^{1}(\mathscr{O}, J)^{\otimes(d i+r)} \otimes\left(\operatorname{Im} \alpha_{J}\right)^{\otimes(q-i)} \otimes \operatorname{gr}_{C}^{0} \mathscr{S} \rightarrow \operatorname{gr}^{n, i}(\mathscr{S}, J) .
$$

For an arbitrary $i \in[0, q]$, one has

(i) $\gamma^{n, i}(\mathscr{S}, J)$ is injective at smooth points of $C$, and 
(ii) $\operatorname{Im} \alpha_{J}=\operatorname{Ker} \beta_{J}$ and $\gamma^{n, i}(\mathscr{S}, J)$ is an isomorphism on the open dense subset of $C$ consisting of the points $P$ at which $\mathscr{S}$ is a locally free $\mathscr{O}_{X}$-module and $J$ is a nested c.i. Furthermore, if $u, v$ are the elements in $\mathscr{O}_{X, P}$ as in (8.4), then we have

$$
\Phi^{i} \operatorname{gr}^{n}(\mathscr{S}, J)=u^{d i} \operatorname{gr}^{n-\mathrm{id}}(\mathscr{S}, J) \text { for } i \in[0, q] .
$$

Proof. By (8.4(ii)), $\operatorname{Ker} \alpha_{J}$ and $\operatorname{Im} \alpha_{J}$ are pure rank $1 \mathscr{O}_{C}$-modules fitting in the exact sequence $0 \rightarrow \operatorname{Ker} \alpha_{J} \rightarrow \operatorname{gr}_{C}^{0} J \rightarrow \operatorname{Im} \alpha_{J} \rightarrow 0$. Let $\Phi^{i}$ be the subsheaf of $\operatorname{gr}^{n}(\mathscr{S}, J)$ given as the saturation of the image of the map

$$
\left(\operatorname{Ker} \alpha_{J}\right)^{\otimes i} \otimes S^{q-i}\left(\operatorname{gr}^{d}(\mathscr{O}, J)\right) \otimes \operatorname{gr}^{1}(\mathscr{O}, J)^{\otimes r} \otimes \operatorname{gr}_{C}^{0} \mathscr{S} \rightarrow \operatorname{gr}^{n}(\mathscr{S}, J)
$$

induced by $\gamma^{n}(\mathscr{S}, J)$ for $i \in[0, q]$. Then $\Phi^{i}$ is a saturated $\mathscr{O}_{C}$-submodule of pure rank $(q+1-i) \rho$ by $(8.5)$. Since the sequence

$$
\left(\operatorname{Ker} \alpha_{J}\right) \otimes S^{q-i-1} \operatorname{gr}^{d}(\mathscr{O}, J) \stackrel{\delta(i)}{\longrightarrow} S^{q-i} \operatorname{gr}^{d}(\mathscr{O}, J) \rightarrow S^{q-i}\left(\operatorname{Im} \alpha_{J}\right) / \operatorname{Sat}(0) \rightarrow 0
$$

constructed in the obvious way is a 0 -sequence $(\operatorname{Sat}(0)$ is the saturation of 0$)$ and exact on an open dense subset of $C$, one has

$$
S^{q-i}\left(\operatorname{gr}^{d}(\mathscr{O}, J)\right) / \operatorname{Sat}(\operatorname{Im}(\delta(i))) \simeq S^{q-i}\left(\operatorname{Im} \alpha_{J}\right) / \operatorname{Sat}(0) .
$$

Thus by the right exactness of the tensor functor, one has

$$
\left(\operatorname{Ker} \alpha_{J}\right)^{\otimes i} \otimes S^{q-i}\left(\operatorname{Im} \alpha_{J}\right) \otimes \operatorname{gr}^{1}(\mathscr{O}, J)^{\otimes r} \otimes \operatorname{gr}_{C}^{0} \mathscr{S} \rightarrow \Phi^{i} / \Phi^{i+1} .
$$

Using the map $\Delta_{J}: \operatorname{gr}^{1}(\mathscr{O}, J)^{\otimes d} \rightarrow \operatorname{Ker} \alpha_{J}$ induced by $E(J)$, we have $\gamma^{n, i}(\mathscr{S}, J)$. The rest follows from (8.4) as in (8.5), except that the last equality follows from $(8.3(\mathrm{i}))$.

(8.7) Remark. If $X \subset C \simeq \mathbb{P}^{1}$ is an extremal nbd and $J$ is a $C$-laminal ideal of width $d$, then for $\mathscr{S}=\mathscr{O}_{X}, \omega_{X}$, one sees that $\operatorname{gr}^{n}(\mathscr{S}, J)$ is a locally free $\mathscr{O}_{C}$-module of rank $[n / d]+1$. Let $n \in \mathbb{Z}_{+}$. As in (2.3.3) and (2.3.4), one sees that

$$
\chi\left(F^{1}(\mathscr{S}, J) / F^{n+1}(\mathscr{S}, J)\right)=\sum_{1 \leq e \leq n}\left(\operatorname{deg} \operatorname{gr}^{e}(\mathscr{S}, J)+[e / d]+1\right) \geq 0,
$$

and that if $\operatorname{deg} \operatorname{gr}^{e}(\mathscr{S}, J) \leq-[e / d]-1$ for all $e \in[1, n]$, then

$$
\operatorname{gr}^{e}(\mathscr{S}, J) \simeq \mathscr{O}(-1)^{\oplus([e / d]+1)}
$$

for $e \in[1, n]$ and $\operatorname{deg} \operatorname{gr}^{e}(\mathscr{S}, J) \geq-[e / d]-1$ for $e=n+1$.

(8.8) Let $(X, P)$ be the germ of a 3-fold terminal singularity of index $m$ and $C \subset(X, P)$ a reduced curve. Let $\pi^{\sharp}:\left(X^{\sharp}, P^{\sharp}\right) \rightarrow(X, P)$ be the $\boldsymbol{\mu}_{m}$-canonical cover and $C^{\sharp}=\pi^{\sharp-1}(C)_{\text {red }} \subset\left(X^{\sharp}, P^{\sharp}\right)$. Meanings of these symbols will be fixed in paragraphs $(8.8)-(8.8 .6)$. 
Let $\mathscr{S}$ be a coherent sheaf (on $X$, or $X^{\sharp}$ ). A subquotient of $\mathscr{S}$ is a coherent sheaf $\mathscr{L}$ endowed with an identification $\mathscr{L}=\mathscr{M} \mid \mathcal{N}$, where $\mathscr{M}$ and $\mathscr{N}$ are coherent subsheaves of $\mathscr{S}$. The subquotient $\mathscr{L}$ is said to be $\mathscr{S}$-saturated if $\mathscr{S} \supset \mathscr{M} \supset \mathscr{N}$ is saturated. An isomorphism theorem says that an arbitrary subquotient $\mathscr{K}$ of $\mathscr{L}$ is a subquotient of $\mathscr{S}$, and if furthermore $\mathscr{L}$ is $\mathscr{S}$ saturated, then $\mathscr{K}$ is $\mathscr{L}$-saturated $\Leftrightarrow \mathscr{K}$ is $\mathscr{S}$-saturated (8.1.1). Therefore we may simply say, for instance, $\mathscr{L}$ is saturated if there is no danger of confusion.

Let $\operatorname{SSQ}(\mathscr{S})$ be the small category of saturated subquotients of $\mathscr{S}$, where $\operatorname{Hom}\left(\mathscr{M}_{1} / \mathscr{N}_{1}, \mathscr{M} / \mathscr{N}\right)$ consists only of the natural map if $\mathscr{M}_{1} \subset \mathscr{M}$ and $\mathscr{N}_{1} \subset \mathscr{N}$ (or $\varnothing$ if otherwise). If $\mathscr{S}$ is a $\boldsymbol{\mu}_{m}$-sheaf on $X^{\sharp}$, then $\operatorname{SSQ}\left(\mathscr{S}, \boldsymbol{\mu}_{m}\right)$ is the full subcategory of $\operatorname{SSQ}(\mathscr{S})$ consisting of $\mathscr{M} / \mathscr{N}$ such that $\mathscr{M}$ and $\mathscr{N}$ are $\boldsymbol{\mu}_{m^{-}}$ stable subsheaves of $\mathscr{S}$.

Let $\mathscr{S}$ be a coherent sheaf on $X$ such that $\mathscr{S} \supset 0$ is saturated. By an $\ell$-structure of $\mathscr{S}$ at $P$ (or, of $\mathscr{S}_{P}=\mathscr{S} \otimes \mathscr{O}_{X, P}$ ), we mean a coherent sheaf $\mathscr{S}^{\sharp}$ on $X^{\sharp}$ with $\boldsymbol{\mu}_{m}$-action endowed with an isomorphism $\mathscr{S}_{\{0\}}^{\sharp} \simeq \mathscr{S}$ (cf. (2.5)) such that $\mathscr{S}^{\sharp} \supset 0$ is saturated. We note that $\mathscr{O}_{X}$ has a trivial $\ell$-structure $\mathscr{O}_{X}^{\sharp} \supset \mathscr{O}_{X}$.

(8.8.1) Lemma-Definition. Let $\mathscr{S}$ be a coherent sheaf on $X$ with an $\ell$-structure $\mathscr{S}^{\sharp} \supset \mathscr{S}$. Then there is a $1-1$ correspondence between $\operatorname{SSQ}(\mathscr{S})$ and $\operatorname{SSQ}\left(\mathscr{S}^{\sharp}, \mu_{m}\right)$ :

$$
\operatorname{SSQ}(\mathscr{S}) \underset{\text { quo }}{\stackrel{\text { lif }}{\rightleftarrows}} \operatorname{SSQ}\left(\mathscr{S}^{\sharp}, \mu_{m}\right),
$$

where lif and quo are exact functors such that lif $\circ$ quo $=\mathrm{id}$ and quoolif $=\mathrm{id}$, and are defined for subsheaves $\mathscr{M}$ and $\mathscr{M}^{\sharp}$ as $\operatorname{lif}(\mathscr{M})=\operatorname{Sat}_{\mathscr{S}^{\sharp}}\left(\mathscr{O}_{X^{\sharp}} \mathscr{M}\right)$ and quo $\left(\mathscr{M}^{\sharp}\right)=\mathscr{M}_{\{0\}}^{\sharp}=\mathscr{M}^{\sharp} \cap \mathscr{S}$, where $\mathscr{O}_{X^{\sharp}} \mathscr{M}$ is the subsheave of $\mathscr{S}$ generated by $\pi^{\sharp *} \mathscr{M}$. For each saturated subquotient $\mathscr{M}$ of $\mathscr{S}, \operatorname{lif}(\mathscr{M})$ and $\operatorname{lif}(\mathscr{M}) \supset \mathscr{M}$ are called the canonical lifting of $\mathscr{M}$ and the induced $\ell$-structure of $\mathscr{M}$. (If there is a danger of confusion, $\mathscr{S}$-lif $(\mathscr{M})$ will be used. )

Proof. It is clear that lif and quo for subsheaves satisfy lif $\circ$ quo $=$ id and quo $\circ$ lif $=$ id, because $\pi^{\sharp}$ is étale outside $P^{\sharp}$. Therefore the definition of lif (similarly of quo) extends to subquotients $\mathscr{M} / \mathscr{N}$ by exactness: $\operatorname{lif}(\mathscr{M} / \mathscr{N})=$ $\operatorname{lif}(\mathscr{M}) / \operatorname{lif}(\mathscr{N})$.

(8.8.2) Proposition-Definition. Let $\mathscr{L}, \mathscr{M}$ and $\mathscr{N}$ be coherent sheaves on $X$ with $\ell$-structures $\mathscr{L} \subset \mathscr{L}^{\sharp}, \mathscr{M} \subset \mathscr{M}^{\sharp}$, and $\mathscr{N} \subset \mathscr{N}^{\sharp}$. Then

(i) the restriction map

$$
\text { res: } \operatorname{Hom}_{\mu_{m}-\mathscr{O}_{X^{\sharp}}}\left(\mathscr{M}^{\sharp}, \mathscr{N}^{\sharp}\right) \rightarrow \operatorname{Hom}_{\mathscr{O}_{X}}(\mathscr{M}, \mathscr{N})
$$

defined by $\operatorname{res}\left(\phi^{\sharp}\right)=\left.\phi\right|_{\mathscr{M}}$ is an injection. If an $\mathscr{O}_{X}$-homomorphism $\phi: \mathscr{M} \rightarrow \mathscr{N}$ extends to a $\mu_{m}-\mathscr{O}_{X^{\sharp}}$-homomorphism $\phi^{\sharp}: \mathscr{M}^{\sharp} \rightarrow \mathscr{N}^{\sharp}$ in this sense, we say that 
$\phi$ is an $\ell$-homomorphism and that $\phi^{\sharp}$ is the canonical lifting of $\phi$, since it is unique,

(ii) we say that an $\ell$-homomorphism $\phi$ is an $\ell$-surjection (resp. $\ell$-isomorphism, $\ell$-injection) if the canonical lifting $\phi^{\sharp}$ is a surjection (resp. isomorphism, injection). Then $\phi$ is an injection iff it is an $\ell$-injection, and $\phi$ is a

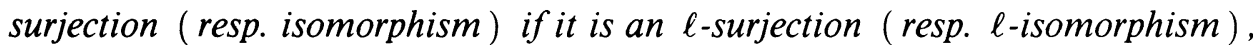

(iii) if $\mathscr{M}=\mathscr{A} \oplus \mathscr{B}$ is a splitting by $\mathscr{O}_{X}$-submodules $\mathscr{A}, \mathscr{B} \subset \mathscr{M}$, then it is called an $\ell$-splitting and written as $M=\mathscr{A} \widetilde{\oplus} \mathscr{B}$ if $\mathscr{M}^{\sharp}=\operatorname{lif}(\mathscr{A}) \oplus \operatorname{lif}(\mathscr{B})$,

(iv) let $\psi: \mathscr{L} \rightarrow \mathscr{M}$ and $\phi: \mathscr{M} \rightarrow \mathscr{N}$ be $\ell$-homomorphisms. Then $\mathscr{E}: 0 \rightarrow$ $\mathscr{L} \stackrel{\psi}{\longrightarrow} \mathscr{M} \stackrel{\phi}{\longrightarrow} \mathscr{N} \rightarrow 0$ is a 0 -sequence iff its canonical lifting $\mathscr{E}^{\sharp}: 0 \rightarrow \mathscr{L}^{\sharp} \stackrel{\psi^{\sharp}}{\longrightarrow}$ $\mathscr{M}^{\sharp} \stackrel{\phi^{\sharp}}{\longrightarrow} \mathscr{N}^{\sharp} \rightarrow 0$ is a 0 -sequence. We say that $\mathscr{E}$ is $\ell$-exact (resp. $\ell$-split) if $\mathscr{E}^{\sharp}$ is exact (resp. split) as a sequence of $\boldsymbol{\mu}_{m}-\mathscr{O}_{X^{\sharp}}$-homomorphisms. Then $\mathscr{E}$ is exact (resp. split) if it is $\ell$-exact (resp. $\ell$-split). We note that these are compatible with the definitions in (ii) and (iii), and

(v) let $\phi: \mathscr{M} \rightarrow \mathscr{N}$ be an $\ell$-homomorphism. If $\phi$ is an $\ell$-surjection (resp. $\ell$-injection and $\mathscr{N} \supset \phi(\mathscr{M})$ is saturated), then $\operatorname{Ker} \phi$ (resp. Coker $\phi)$ has an induced $\ell$-structure and $0 \rightarrow \operatorname{Ker} \phi \rightarrow \mathscr{M} \rightarrow \mathscr{N} \rightarrow 0$ (resp. $0 \rightarrow \mathscr{M} \rightarrow \mathcal{N} \rightarrow$ Coker $\phi \rightarrow 0)$ is $\ell$-exact.

Proof. (i) The map res is well defined because $\phi^{\sharp}(\mathscr{M}) \subset \mathscr{N}_{\{0\}}^{\sharp}=\mathscr{N}$. It is injective because $\mathscr{M}^{\sharp} \supset 0$ is saturated and $\left.\phi^{\sharp}\right|_{X^{\sharp}-\left\{P^{\sharp}\right\}}=\left(\left.\pi^{\sharp}\right|_{X-\{P\}}\right)^{*} \phi$ if $\phi=$ $\operatorname{res}\left(\phi^{\sharp}\right)$, which follows from the fact that $X^{\sharp}-\left\{P^{\sharp}\right\} \rightarrow X-\{P\}$ is a finite étale $\boldsymbol{\mu}_{m}$-Galois covering.

(ii) If $\operatorname{Ker} \phi^{\sharp} \neq 0$ then $\operatorname{Supp}\left(\operatorname{Ker} \phi^{\sharp}\right) \not \subset\left\{P^{\sharp}\right\}$ by $P^{\sharp} \notin \operatorname{Ass}\left(\mathscr{M}^{\sharp}\right)$, whence $\operatorname{Supp}(\operatorname{Ker} \phi) \not \subset\{P\}$ by flat descent. Thus $\phi$ is an $\ell$-injection if it is an injection. Other assertions are obvious because $\boldsymbol{\mu}_{m}$ is linearly reductive.

(iv) Since $\operatorname{res}\left(\phi^{\sharp} \circ \psi^{\sharp}\right)=\phi \circ \psi$, one sees $\phi \circ \psi=0 \Leftrightarrow \phi^{\sharp} \circ \psi^{\sharp}=0$ by (i). This proves the first assertion, and others follow from the linear reductivity of $\boldsymbol{\mu}_{m}$.

(v) By the assumptions, $\operatorname{Ker} \phi$ (resp. Coker $\phi$ ) is a saturated subquotient of $\mathscr{M}$ whence has an induced $\ell$-structure. Since $0 \rightarrow \operatorname{Ker} \phi^{\sharp} \rightarrow \mathscr{M}^{\sharp} \rightarrow \mathscr{N}^{\sharp} \rightarrow 0$ (resp. $0 \rightarrow \mathscr{M}^{\sharp} \rightarrow \mathscr{N}^{\sharp} \rightarrow$ Coker $\phi^{\sharp} \rightarrow 0$ ) is exact, the assertion follows by (iv).

(8.8.3) Definition. Let $\mathscr{M}$ be a coherent sheaf on $X$ with $\ell$-structure $\mathscr{M} \subset$ $\mathscr{M}^{\sharp}$. If $s_{1}^{\sharp}, \ldots, s_{r}^{\sharp} \in \mathscr{M}^{\sharp}$ are $\boldsymbol{\mu}_{m}$-semi-invariants generating $\mathscr{M}^{\sharp}$ as $\mathscr{O}_{X^{\sharp}}$-module at $P^{\sharp}$, we say that $\left\{s_{1}^{\sharp}, \ldots, s_{r}^{\sharp}\right\}$ is an $\ell$-basis of $\mathscr{M}$ at $P$ (or, of $\mathscr{M}_{P}$ ).

Let $Y$ be a closed subscheme of $X$ such that $P \notin \operatorname{Ass}\left(\mathscr{O}_{Y}\right)$ and let $Y^{\sharp}=$ $\operatorname{lif}(Y)$. Then it is easy to see that $\mathscr{M}$ is an $\mathscr{O}_{Y}$-module iff $\mathscr{M}^{\sharp}$ is an $\mathscr{O}_{Y^{\sharp}}$-module. We say that $\mathscr{M}$ is an $\ell$-free $\mathscr{O}_{Y}$-module at $P$ iff $\mathscr{M}^{\sharp}$ is a free $\mathscr{O}_{Y^{\sharp}}$-module at $P^{\sharp}$. If $\mathscr{M}$ is an $\ell$-free $\mathscr{O}_{Y}$-module at $P$, then an $\ell$-basis of $\mathscr{M}$ at $P$ is said to be $\ell$-free if it is a free $\mathscr{O}_{Y^{\sharp}}$-basis. We say that an $\ell$-free $\mathscr{O}_{Y}$-module is $\ell$-trivial 
if it has an $\ell$-free $\ell$-basis consisting of $\mu_{m}$-invariants. We note that $\ell$-trivial $\ell$-free $\mathscr{O}_{Y}$-modules are free, but in general, there is no implication between freeness and $\ell$-freeness in either direction. Therefore, we call a free $\ell$-free $\mathscr{O}_{Y^{-}}$ module at $P$ a $q \ell$-free $\mathscr{O}_{Y}$-module. We call $\ell$-free (resp. $q \ell$-free) $\mathscr{O}_{Y}$-modules at $P$ of rank $1 \ell$-invertible (resp. q $\ell$-invertible) $\mathscr{O}_{Y}$-modules at $P$.

(8.8.4) Let $Y, Y^{\sharp}$ be as above, and $Z$ a closed subscheme of $Y$ such that $P \notin$ $\operatorname{Ass}(Z)$. Let $\mathscr{L}, \mathscr{M}, \mathscr{N}$ be $\mathscr{O}_{Y}$-modules at $P$ with $\ell$-structures $\mathscr{L} \subset \mathscr{L}^{\sharp}$, $\mathscr{M} \subset \mathscr{M}^{\sharp}, \mathscr{N} \subset \mathscr{N}^{\sharp}$. We list the following obvious operations together with easy comments.

$(\widetilde{\oplus}) \mathscr{M} \widetilde{\oplus} \mathscr{N} \subset(\mathscr{M} \widetilde{\oplus} \mathscr{N})^{\sharp}$ is an $\mathscr{O}_{Y}$-module at $P$ with $\ell$-structure defined by

$$
(\mathscr{M} \tilde{\oplus} \mathscr{N})^{\sharp}=\mathscr{M}^{\sharp} \oplus \mathscr{N}^{\sharp} \text {. }
$$

We note that this is compatible with $\ell$-splitting in (8.8.2(iii)), and we have natural $\ell$-isomorphisms

$$
\begin{aligned}
(\mathscr{L} \widetilde{\oplus} \mathscr{M}) \widetilde{\oplus} & \simeq \mathscr{L} \widetilde{\oplus}(\mathscr{M} \widetilde{\oplus}), \\
\mathscr{M} \widetilde{\oplus} \mathscr{N} & \simeq \mathscr{N} \widetilde{\oplus} \mathscr{M} .
\end{aligned}
$$

Let $\chi$ be an $\ell$-character (2.5) for $X \supset C$ at $P$. Let $\mathscr{L}_{Y}[\nu]$, for $\nu \in \mathbb{Z}_{m}$, be the $\ell$-invertible $\mathscr{O}_{Y}$-module with an $\ell$-basis $e_{\nu}$ on which $\mu_{m}$ acts by $\zeta\left(e_{\nu}\right)=$ $\chi(\zeta)^{\nu} e_{\nu}\left(\zeta \in \mu_{m}\right)$. Then $\ell$-free $\mathscr{O}_{Y}$-module $\mathscr{M}$ of rank $r$ is determined by wt (say, $\nu_{1}, \ldots, \nu_{r} \bmod m$ ) of its $\ell$-free $\ell$-basis, that is there is an $\ell$-isomorphism $\mathscr{M} \simeq \mathscr{L}_{Y}\left[\nu_{1}\right] \widetilde{\oplus} \cdots \widetilde{\oplus} \mathscr{L}_{Y}\left[\nu_{r}\right]$. Hence there are at most $\left(\begin{array}{c}m \\ r\end{array}\right) \quad \ell$-free rank $r$ $\mathscr{O}_{Y}$-modules. We note that $\mathscr{M}$ is $q \ell$-free iff $\mathscr{L}_{Y}\left[\nu_{i}\right]$ is $q \ell$-free for all $i \in[1, r]$ by $(8.8 .2(i i))$, and that $\mathscr{M}$ is $\ell$-trivial iff $\nu_{i} \equiv 0(\bmod m)$ for all $i \in[1, r]$.

$(\widetilde{\otimes}) \mathscr{M} \widetilde{\otimes} \mathscr{N} \subset(\mathscr{M} \widetilde{\otimes} \mathscr{N})^{\sharp}$ is an $\mathscr{O}_{Y}$-module at $P$ with $\ell$-structure defined by

$$
(\mathscr{M} \tilde{\otimes} \mathscr{N})^{\sharp}=\left(\mathscr{M}^{\sharp} \otimes_{\mathscr{O}_{X^{\sharp}}} \mathscr{N}^{\sharp}\right) / \text { Sat }_{\mathscr{M}^{\sharp} \otimes \mathscr{N}^{\sharp}}(0) .
$$

It is a biadditive functor in the obvious sense, and we have natural $\ell$-isomorphisms.

$$
\begin{gathered}
\mathscr{M} \tilde{\otimes} \mathscr{O}_{Y} \simeq \mathscr{M} \quad\left(\mathscr{O}_{Y} \text { denotes the } \ell \text {-trivial } \mathscr{O}_{Y}\right), \\
(\mathscr{L} \tilde{\otimes} \mathscr{M}) \widetilde{\otimes} \mathscr{N} \simeq \mathscr{L} \widetilde{\otimes}(\mathscr{M} \tilde{\otimes} \mathscr{N}), \\
\mathscr{M} \widetilde{\otimes} \mathscr{N} \simeq \mathscr{N} \widetilde{\otimes} \mathscr{M} \\
\mathscr{L} \tilde{\otimes}(\mathscr{M} \tilde{\oplus} \mathscr{N}) \simeq(\mathscr{L} \widetilde{\otimes} \mathscr{M}) \tilde{\oplus}(\mathscr{L} \tilde{\otimes} \mathscr{N}),
\end{gathered}
$$

and easy comments

( $\widetilde{\otimes} .4) \mathscr{L} \widetilde{\otimes} \alpha$ is $\ell$-surjective if $\alpha: \mathscr{M} \rightarrow \mathscr{N}$ is $\ell$-surjective,

$(\widetilde{\otimes} .5) \mathscr{L} \widetilde{\otimes} \mathscr{E}^{\circ}$ is $\ell$-exact if $\mathscr{L}$ is $\ell$-free and $\mathscr{E}^{\circ}$ is $\ell$-exact,

$\left(\widetilde{\otimes}\right.$.6) $\mathscr{M} \widetilde{\otimes} \mathscr{N}$ is an $\ell$-free $\mathscr{O}_{Y}$-module at $P$ of rank $r \cdot s$ if $\mathscr{M}$ and $\mathscr{N}$ are $\ell$-free $\mathscr{O}_{Y}$-modules at $P$ of rank $r$ and $s$,

$(\widetilde{\otimes} .7) \mathscr{M} \widetilde{\otimes} \mathscr{O}_{Z}$ is an $\ell$-free $\mathscr{O}_{Z}$-module at $P$ if $\mathscr{M}$ is an $\ell$-free $\mathscr{O}_{Y}$-module at $P$, 
$(\widetilde{\otimes} .8) \mathscr{M} \widetilde{\otimes} \mathscr{N} \simeq \mathscr{M} \otimes \mathscr{N}$ as $\mathscr{O}_{Y}$-modules if $\mathscr{M}$ is $\ell$-trivial, and

$(\widetilde{\otimes} .9)$ the natural $\mathscr{O}_{X}$-homomorphism $\mathscr{M} \otimes_{\mathscr{O}_{X}} \mathscr{N} \rightarrow \mathscr{M} \widetilde{\otimes} \mathscr{N}$ is an isomorphism outside $P$.

$(\tilde{\otimes} \widetilde{\otimes}) \quad \ell$-invertible $\mathscr{O}_{Y}$-module $\mathscr{M}^{\widetilde{\otimes} n} \subset\left(\mathscr{M}^{\widetilde{\otimes} n}\right)^{\sharp}$ at $P$ is defined for $n \in \mathbb{Z}$ and $\ell$-invertible $\mathscr{O}_{Y}$-module $\mathscr{M}$ at $P$ by $\left(\mathscr{M}^{\widetilde{\otimes} n}\right)^{\sharp}=\left(\mathscr{M}^{\sharp}\right)^{\otimes n}$. We have natural $\ell$-isomorphisms

$$
\begin{aligned}
& \mathscr{L}_{Y}[\nu]^{\widetilde{\otimes} n} \simeq \mathscr{L}_{Y}[n \nu] \quad\left(n \in \mathbb{Z}, \nu \in \mathbb{Z}_{m}\right), \\
& \mathscr{M}^{\widetilde{\otimes} a} \widetilde{\otimes} \mathscr{M}^{\widetilde{\otimes} b} \simeq \mathscr{M}^{\widetilde{\otimes}(a+b)} \quad(a, b \in \mathbb{Z}),
\end{aligned}
$$

whence $\ell$-invertible $\mathscr{O}_{Z}$-modules $\left(\mathscr{O}_{Z} \tilde{\otimes} \mathscr{M}^{\tilde{\otimes} n}\right)$ at $P(\tilde{\otimes} .7)$ satisfy

$$
\left(\mathscr{O}_{Z} \tilde{\otimes} \mathscr{M}\right)^{\widetilde{\otimes} n} \simeq \mathscr{O}_{Z} \widetilde{\otimes}\left(\mathscr{M}^{\widetilde{\otimes} n}\right) \quad(n \in \mathbb{Z})
$$

by $(\tilde{\otimes} .1),(\tilde{\otimes} .2)$ and $\mathscr{O}_{Z} \tilde{\otimes}_{Z} \simeq \mathscr{O}_{Z}(\widetilde{\otimes} .0)$. We note by $(\tilde{\otimes} \widetilde{\otimes} .1)$ that

$(\tilde{\otimes} \widetilde{\otimes} .4) \mathscr{M}^{\tilde{\otimes} a}$ is $\ell$-trivial if $a \equiv 0(\bmod m)$,

whence we see by $(\tilde{\otimes} .8)$ that

$(\tilde{\otimes} \tilde{\otimes} .5) \quad(\mathscr{M} \widetilde{\otimes} \mathscr{N})^{\otimes} \tilde{\otimes} n \simeq\left(\mathscr{M}^{\widetilde{\otimes} m}\right)^{\otimes n} \otimes\left(\mathscr{N}^{\widetilde{\otimes} m}\right)^{\otimes n}$ as $\mathscr{O}_{Y}$-modules for $n \in \mathbb{Z}$, if $\mathscr{N}$ is also an $\ell$-invertible $\mathscr{O}_{Y}$-module at $P$.

( $\widetilde{\text { det}}) \ell$-invertible $\mathscr{O}_{Y}$-module $\widetilde{\operatorname{det}} \mathscr{M} \subset(\widetilde{\operatorname{det}} \mathscr{M})^{\sharp}$ at $P$ is defined for $\ell$-free $\mathscr{O}_{Y}$-module $\mathscr{M}$ at $P$ by $(\widetilde{\operatorname{det}} \mathscr{M})^{\sharp}=\operatorname{det}_{\mathscr{O}_{X}} \mathscr{M}^{\sharp}$.

$(\widetilde{S}) \ell$-free $\mathscr{O}_{Y}$-module $\widetilde{S}^{n}(\mathscr{M}) \subset \widetilde{S}^{n}(\mathscr{M})^{\sharp}$ at $P$ is defined for $n \in \mathbb{N}$ and $\ell$-free $\mathscr{O}_{Y}$-module $\mathscr{M}$ at $P$ by $\widetilde{S}^{n}(\mathscr{M})^{\sharp}=S_{\mathscr{O}_{X \sharp}}\left(\mathscr{M}^{\sharp}\right)$ (symmetric $n$th power). If $\mathscr{M}$ and $\mathscr{N}$ are $\ell$-free $\mathscr{O}_{Y}$-modules of rank 2 and 1 respectively, then we have a natural $\ell$-isomorphism for $n \in \mathbb{N}$

$$
\widetilde{\operatorname{det}}\left(\widetilde{S}^{n}(\mathscr{M}) \widetilde{\otimes} \mathscr{N}\right) \simeq(\widetilde{\operatorname{det}} \mathscr{M})^{\widetilde{\otimes} n(n+1) / 2} \widetilde{\otimes} \mathscr{N}^{\widetilde{\otimes}(n+1)} .
$$

(8.8.5) If furthermore $\operatorname{dim} Y=1$ and $\mathscr{M}$ is $q \ell$-free, then one can define local degree $q l \operatorname{deg}(\mathscr{M}, P) \in \mathbb{Z}_{+}$as follows: let $s_{1}, \ldots, s_{r}$ (resp. $s_{1}^{\sharp}, \ldots, s_{r}^{\sharp}$ ) be a free basis (resp. an $\ell$-free basis) of $\mathscr{M}$ at $P$. Then $s_{i}=\sum_{j} f_{i j} s_{j}^{\sharp} \quad(i \in[1, r])$ and $g=\operatorname{det}\left(f_{i j}\right)$ is a semi-invariant by $s_{1} \wedge \cdots \wedge s_{r}=g\left(s_{1}^{\sharp} \wedge \cdots \wedge s_{r}^{\sharp}\right)$, whence $g^{m} \in$ $\mathscr{O}_{Y, P}$. Then $\mathrm{gl} \operatorname{deg}(\mathscr{M}, P)=\operatorname{len}_{P}\left(\mathscr{O}_{Y, P} /\left(g^{m}\right)\right)$, which is obviously independent of choice of $\ell$-basis and basis.

If we set $B(Y, P)=\operatorname{Max}\left\{q l \operatorname{deg}\left(\mathscr{L}_{Y}[\nu], P\right) \mid \nu \in \mathbb{Z}_{m}, \mathscr{L}_{Y}[\nu]: q \ell\right.$-free $\}$, then $q l \operatorname{deg}(\mathscr{M}, P) \leq B(Y, P) \cdot r$ for arbitrary $q \ell$-free $\mathscr{M}$ of rank $r$.

We note that if $Y$ is a smooth curve at $P$ and if $\mathscr{M}$ is a $q \ell$-free (i.e. $\ell$-free) $\mathscr{O}_{C}$-module at $P$ with an $\ell$-free $\ell$-basis $\left(s_{1}^{\sharp}, \ldots, s_{r}^{\sharp}\right)$, then

$$
q l \operatorname{deg}(\mathscr{M}, P)=\sum_{i} R\left(Y^{\sharp}-\mathrm{wt} s_{i}^{\sharp}\right)
$$

(cf. (2.8)). 
As a corollary to these definitions, we have

(8.8.6) Corollary. Let $J$ be a C-laminal ideal of width $d$ and $\mathscr{S} \subset \mathscr{S}^{\sharp}$ an $\ell$-free $\mathscr{O}_{X}$-module at $P$. Then

(i) $\mathscr{O}_{C^{\sharp}}=\mathscr{O}_{X}$-lif $\left(\mathscr{O}_{C}\right) \quad$ (that is $\left.C^{\sharp}=\operatorname{lif}(C)\right), J^{\sharp}=\mathscr{O}_{X}$-lif $(J)$ is a $C^{\sharp}$-laminal ideal of width $d$, we have equalities of subquotients

$$
F^{n}\left(\mathscr{S}^{\sharp}, J^{\sharp}\right)=\mathscr{S}-\operatorname{lif} F^{n}(\mathscr{S}, J), \quad \operatorname{gr}^{n}\left(\mathscr{S}^{\sharp}, J^{\sharp}\right)=\mathscr{S}-\operatorname{lif}_{\operatorname{gr}^{n}}(\mathscr{S}, J)
$$

and hence $\ell$-isomorphisms

$$
F^{n}(\mathscr{S}, J) \simeq F^{n}(\mathscr{O}, J) \widetilde{\otimes} \mathscr{S} \text { and } \operatorname{gr}^{n}(\mathscr{S}, J) \simeq \operatorname{gr}^{n}(\mathscr{O}, J) \widetilde{\otimes} \mathscr{S}
$$

for all $n \geq 0$. If furthermore $d \geq 2$, then similarly we have

$$
\operatorname{gr}^{n, i}\left(\mathscr{S}^{\sharp}, J^{\sharp}\right)=\mathscr{S}-\operatorname{lif} \operatorname{gr}^{n, i}(\mathscr{S}, J), \quad \operatorname{gr}^{n, i}(\mathscr{S}, J) \simeq \operatorname{gr}^{n, i}(\mathscr{O}, J) \widetilde{\otimes}
$$

for all $n, i$ (8.6), and

(ii) homomorphisms $\alpha_{J^{\sharp}}, \beta_{J^{\sharp}}, \Delta_{J^{\sharp}}(8.2 .2), \gamma^{n}\left(\mathscr{S}^{\sharp}, J^{\sharp}\right)\left(\right.$ and $\gamma^{n, i}\left(\mathscr{S}^{\sharp}, J^{\sharp}\right)$ if $d \geq 2$ ) are $\boldsymbol{\mu}_{m}$-homomorphisms "lifting" $\alpha_{J}, \beta_{J}, \Delta_{J}, \gamma^{n}(\mathscr{S}, J)$ (and $\gamma^{n, i}(\mathscr{S}, J)$ if $\left.d \geq 2\right)$ in the obvious sense. In particular, $\alpha_{J}$ is an $\ell$-homomorphism, $\beta_{J}$ is an $\ell$-surjection, $\operatorname{Ker} \beta_{J} \in \operatorname{SSB}\left(\mathscr{O}_{X}\right), \operatorname{Ker} \beta_{J^{\sharp}}=\operatorname{lif}\left(\operatorname{Ker} \beta_{J}\right)$, $\gamma^{n}(\mathscr{S}, J)=\gamma^{n}(\mathscr{O}, J) \widetilde{\otimes} \mathscr{S}\left(\right.$ and $\gamma^{n, i}(\mathscr{S}, J)=\gamma^{n, i}(\mathscr{O}, J) \widetilde{\otimes} \mathscr{S}$ if $\left.d \geq 2\right)$.

Proof. Equalities in (i) are for saturated subquotients and they hold outside $P^{\sharp}$ $((8.3(\mathrm{i}))$ and $(8.6(\mathrm{ii})))$ where $\pi^{\sharp}$ is étale. Therefore they hold in a nbd of $P^{\sharp}$. The meaning of "lifting" in (ii) is the following commutative diagram

$$
\begin{aligned}
& 0 \rightarrow \operatorname{gr}^{1}(\mathscr{O}, J)^{\otimes d} \stackrel{\Delta_{J}}{\longrightarrow} \operatorname{gr}_{C}^{0}(J) \stackrel{\alpha_{J}}{\longrightarrow} \operatorname{Ker}\left[\operatorname{gr}_{C}^{1} \mathscr{O} \stackrel{\beta_{J}}{\longrightarrow} \operatorname{gr}^{1}(\mathscr{O}, J)\right] \rightarrow 0 \\
& \downarrow \quad \Omega \quad \Omega \\
& 0 \rightarrow \operatorname{gr}^{1}\left(\mathscr{O}^{\sharp}, J^{\sharp}\right) \otimes d \stackrel{\Delta_{J}^{\sharp}}{\longrightarrow} \operatorname{gr}_{C^{\sharp}}^{0}\left(J^{\sharp}\right) \stackrel{\alpha_{J}^{\sharp}}{\longrightarrow} \operatorname{Ker}\left[\operatorname{gr}_{C^{\sharp}}^{1} \mathscr{O}^{\sharp} \stackrel{\beta_{J^{\sharp}}}{\longrightarrow} \operatorname{gr}^{1}\left(\mathscr{O}^{\sharp}, J^{\sharp}\right) \rightarrow 0\right.
\end{aligned}
$$

where $\beta_{J \sharp}$ is surjective. The rest follows from the construction and definition.

(8.9) Definition. Let $X \supset C$ be an extremal nbd and $Y \subset X$ a closed subspace without embedded or isolated points. Let $P_{1}, \ldots, P_{t} \in X$ be points of indices $m_{1}, \ldots, m_{t}(\geq 1)$ such that $X-\left\{P_{1}, \ldots, P_{t}\right\}$ has no points of indices $>1$, and $Y$ is given the induced $\ell$-structure at these points. A coherent $\mathscr{O}_{Y}$-module is said to be $\ell$-coherent if it is given $\ell$-structure at each of $P_{1}, \ldots, P_{t}$. If an $\ell$-coherent $\mathscr{O}_{Y}$-module $\mathscr{M}$ is locally free of rank $r$ on $Y^{\circ}=Y-\left\{P_{1}, \ldots, P_{t}\right\}$ and $\ell$-free (resp. $q \ell$-free) at each $P_{i}$, then we say that $\mathscr{M}$ is a locally $\ell$ free (resp. $q \ell$-free) $\mathscr{O}_{Y}$-module of rank $r$, and we may also say that $\mathscr{M}$ is an $\ell$-invertible (resp. $q \ell$-invertible) $\mathscr{O}_{Y}$-module if $r=1$. Local definitions of $\widetilde{\oplus}$ and $\widetilde{\otimes}$ for $\ell$-coherent $\mathscr{O}_{Y}$-modules (cf. $(\widetilde{\otimes} .9)$ in (8.8.4)), powers for $\ell$ invertible sheaves, $\widetilde{S}^{n}$ and $\widetilde{\mathrm{det}}$ for $\ell$-free $\mathscr{O}_{Y}$-modules $(8.8 .4)$ patch with the corresponding operations on $Y^{\circ}$, and thus these operations give $\ell$-coherent $\mathscr{O}_{Y}$-modules. Formulae in (8.8.4) extending the usual ones to $\mathscr{O}_{Y}$-modules with $\ell$-structures at $P_{i}$ 's extend further to $\ell$-coherent $\mathscr{O}_{Y}$-modules since they coincide with the classical ones on the punctured nbds. Therefore we will quote the local 
formulae in (8.8.4) in global setting by abuse of language. For example, $\operatorname{gr}_{C}^{0} \omega \simeq$ $\mathscr{O}_{C} \widetilde{\otimes} \omega_{X}$ is an $\ell$-invertible $\mathscr{O}_{C}$-module by $(\widetilde{\otimes} .7)$ in (8.8.4) and (8.8.6(i)).

The notion of $\ell$-homomorphism, $\ell$-injection, $\ell$-surjection, $\ell$-isomorphism, $\ell$-splitting, and $\ell$-exactness for $\ell$-coherent sheaves are defined in the obvious way since these are local properties. An $\ell$-exact sequence $0 \rightarrow \mathscr{L} \rightarrow \mathscr{M} \rightarrow$ $\mathscr{N} \rightarrow 0$ is (globally) $\ell$-split if $\mathscr{M}$ has a coherent subsheaf $\mathscr{K}$ giving an $\ell$ splitting $\mathscr{M}=\operatorname{Im}(\mathscr{L} \rightarrow \mathscr{M}) \widetilde{\oplus} \mathscr{K}$.

We note that $\ell$-structure $(\ell$-coherence, $\ldots)$ does not depend on choice of above $P_{1}, \ldots, P_{t}$ as long as $X-\left\{P_{1}, \ldots, P_{t}\right\}$ has no points of indices $>1$.

(8.9.1) Proposition-Definition. Assume further that $Y$ is a complete pure 1-dimensional closed subscheme of $X$. Then

(i) if $\mathscr{M}$ is a locally $\ell$-free $\mathscr{O}_{Y}$-module, then $(\widetilde{\operatorname{det}} \mathscr{M})^{\widetilde{\otimes} \mu}$ is q $\ell$-invertible and

$$
(\widetilde{\operatorname{det}} \mathscr{M})^{\widetilde{\otimes} \mu n} \simeq\left((\widetilde{\operatorname{det}} \mathscr{M})^{\widetilde{\otimes} \mu}\right)^{\otimes n}
$$

as $\mathscr{O}_{Y}$-modules for all $n, \mu \in \mathbb{N}$ such that $\operatorname{lcm}\left\{m_{1}, \ldots, m_{r}\right\} \mid \mu$. Then we define

$$
l \operatorname{deg}_{Y} \mathscr{M}=\frac{1}{\mu} \operatorname{deg}_{Y}(\widetilde{\operatorname{det}} \mathscr{M})^{\widetilde{\otimes} \mu} \in \mathbb{Q}
$$

which is independent of choice of such $\mu$, where $\operatorname{deg}_{Y}$ is the usual degree for $\mathscr{O}_{Y}$-invertible sheaves. We note that $l \operatorname{deg}_{C}\left(\operatorname{gr}_{C}^{0} \omega\right)=\left(\omega_{X} \cdot C\right)$, and

$$
l \operatorname{deg}_{Y}(\mathscr{M} \widetilde{\otimes} \mathscr{N})=l \operatorname{deg}_{Y} \mathscr{M}+l \operatorname{deg}_{Y} \mathscr{N}
$$

if $\mathscr{M}$ and $\mathscr{N}$ are of rank 1 , and

$$
l \operatorname{deg}_{Y}\left(\mathscr{N} \tilde{\otimes} S^{n} \mathscr{M}\right)=\frac{1}{2} n(n+1) l \operatorname{deg}_{Y} \mathscr{M}+(n+1) l \operatorname{deg}_{Y} \mathscr{N} \quad(n \geq 0)
$$

if $\mathscr{M}$ is of rank 2 and $\mathscr{N}$ is of rank 1 ,

(ii) let $Q L(Y)=\mathbb{Z} \oplus\left(\bigoplus_{1 \leq i \leq t} \mathbb{Z} P_{i}^{\sharp}\right) /\left(\sum_{1 \leq i \leq t} \mathbb{Z}\left(1-m_{i} P_{i}^{\sharp}\right)\right)$, then

$$
\operatorname{deg}: Q L(Y) \rightarrow \mathbb{Q} ; \quad \operatorname{deg}\left(z+\sum z_{i} P_{i}^{\sharp}\right)=z+\sum z_{i} / m_{i}
$$

is a well-defined homomorphism. For a locally q $\ell$-free $\mathscr{O}_{Y}$-module $\mathscr{M}$, let

$$
q l_{Y}(\mathscr{M})=\operatorname{deg}_{Y} \mathscr{M}+\sum_{i=1}^{t} q l \operatorname{deg}\left(\mathscr{M}, P_{i}\right) P_{i}^{\sharp} \in Q L(Y),
$$

then $l \operatorname{deg}_{Y} \mathscr{M}=\operatorname{deg}\left(q l_{Y}(\mathscr{M})\right) ;$ and $q l_{Y}(\mathscr{M} \tilde{\otimes} \mathscr{N})=q l_{Y}(\mathscr{M})+q l_{Y}(\mathscr{N})$ for q $\ell$-invertible $\mathscr{M}$ and $\mathscr{N}$ such that $\mathscr{M} \widetilde{\otimes} \mathscr{N}$ is q $\ell$-invertible; and

$$
0 \leq l \operatorname{deg}_{Y} \mathscr{M}-\operatorname{deg}_{Y} \mathscr{M} \leq r \sum_{1 \leq i \leq t} B\left(Y, P_{i}\right) / m_{i}
$$

(cf. (8.8.5)) if $\mathscr{M}$ is of rank $r$, and

(iii) if $Y \simeq \mathbb{P}^{1}$ and $X \supset Y$ is locally primitive, then

$$
T L: Q L(Y) \rightarrow \mathbb{Z} ; \quad T L\left(z+\sum z_{i} P_{i}^{\sharp}\right)=z-\sum U_{i}\left(-z_{i}\right)
$$


is well defined, where $U_{i}$ is defined for $\left(X, P_{i}\right) \supset\left(Y, P_{i}\right)$ as in (2.8). Furthermore, if $\mathscr{M}$ and $\mathscr{N}$ are $\ell$-invertible $\mathscr{O}_{Y}$-modules, then $\operatorname{deg}_{Y} \mathscr{M}=T L\left(q l_{Y}(\mathscr{M})\right)$, whence

$$
\operatorname{deg}_{Y}\left(\mathscr{M}^{\tilde{\otimes} a} \tilde{\otimes} \mathscr{N}^{\tilde{\otimes} b}\right)=T L\left(a q l_{Y}(\mathscr{M})+b q l_{Y}(\mathcal{N})\right) \quad(a, b \in \mathbb{Z}) .
$$

We say that an expression $\zeta=z+\sum_{i} z_{i} P_{i}^{\sharp}$ of $\zeta \in Q L(Y)$ is normalized if $U_{i}\left(-z_{i}\right)=0$ for all $i$.

Proof. (i) The first assertion follows from $(\tilde{\otimes} \widetilde{\otimes} .5)$ in (8.8.4). By $\ell$-isomorphism $\mathrm{gr}_{C}^{0} \omega \simeq \mathscr{O}_{C} \tilde{\otimes} \omega_{X}$ in (8.9), one also has

$$
l \operatorname{deg}_{C}\left(\operatorname{gr}_{C}^{0} \omega\right)=\frac{1}{\mu} \operatorname{deg}_{C}\left(\mathscr{O}_{C} \widetilde{\otimes} \omega_{X}\right)^{\widetilde{\otimes} \mu} \cdot=\frac{1}{\mu} \operatorname{deg}_{C} \mathscr{O}_{C} \otimes\left(\omega_{X}{ }^{\widetilde{\otimes} \mu}\right)=\left(\omega_{X} \cdot C\right) .
$$

The last two assertions follow from the definition by $(\mathscr{M} \widetilde{\otimes} \mathscr{N})^{\widetilde{\otimes} \mu} \simeq\left(\mathscr{M}^{\widetilde{\otimes} \mu}\right) \otimes$ $\left(\mathscr{N}^{\widetilde{\otimes} \mu}\right)$, and $(\widetilde{\otimes} \widetilde{\otimes} .5)$ and $(\widetilde{S} .1)$ in (8.8.4).

(ii) The definition of $q l \operatorname{deg}\left(\mathscr{M}, P_{i}\right)$ shows

$$
\frac{\mu}{m_{i}} \cdot q l \operatorname{deg}\left(\mathscr{M}, P_{i}\right)=\operatorname{len}_{P_{i}} \operatorname{Coker}\left[(\operatorname{det} \mathscr{M})^{\otimes \mu} \rightarrow(\widetilde{\operatorname{det}} \mathscr{M})^{\widetilde{\otimes} \mu}\right]
$$

for locally $q \ell$-free $\mathscr{M}$ if $m_{i} \mid \mu$. By summing up $(*)_{\mathscr{M}, 1}, \ldots,(*)_{\mathscr{M}, t}$, one has $l \operatorname{deg}_{Y} \mathscr{M}=\operatorname{deg}\left(q l_{Y}(\mathscr{M})\right)$. Let $\mathscr{M}$ and $\mathscr{N}$ be $q$ l-invertible $\mathscr{O}_{Y}$-modules such that $\mathscr{M} \tilde{\otimes} \mathscr{N}$ is $q \ell$-invertible. By $(\tilde{\otimes} \tilde{\otimes} .5)$ in (8.8.4) and $(*)_{\mathscr{M}, i}+(*)_{\mathscr{N}, i}-$ ${ }^{(*)} \tilde{M} \widetilde{\otimes} \mathscr{N}, i$, one has

$$
\begin{aligned}
\frac{\mu}{m_{i}}\left(q l \operatorname{deg}\left(\mathscr{M}, P_{i}\right)+q l \operatorname{deg}\left(\mathscr{N}, P_{i}\right)-q l \operatorname{deg}\left(\mathscr{M} \widetilde{\otimes} \mathscr{N}, P_{i}\right)\right) \\
\quad=\operatorname{len}_{P_{i}} \operatorname{Coker}\left[(\mathscr{M} \otimes \mathscr{N})^{\otimes \mu} \rightarrow(\mathscr{M} \widetilde{\otimes} \mathscr{N})^{\widetilde{\otimes} \mu}\right] .
\end{aligned}
$$

Let $a_{i}$ be the length of $\mathscr{M} \tilde{\otimes} \mathscr{N} / \mathscr{M} \otimes \mathscr{N}$ at $P_{i}$. Then

$$
\operatorname{deg}_{Y} \mathscr{M} \tilde{\otimes} \mathscr{N}=\operatorname{deg}_{Y} \mathscr{M} \otimes \mathscr{N}+a_{1}+\cdots+a_{t},
$$

and

$$
q l \operatorname{deg}\left(\mathscr{M}, P_{i}\right)+q l \operatorname{deg}\left(\mathscr{N}, P_{i}\right)=q l \operatorname{deg}\left(\mathscr{M} \widetilde{\otimes} \mathscr{N}, P_{i}\right)+a_{i} m_{i}
$$

Thus

$$
\begin{aligned}
q l_{Y}(\mathscr{M})+q l_{Y}(\mathscr{N}) & =\operatorname{deg}_{Y} \mathscr{M} \otimes \mathscr{N}+\sum_{i}\left(q l \operatorname{deg}\left(\mathscr{M}, P_{i}\right)+q l \operatorname{deg}\left(\mathscr{N}, P_{i}\right)\right) P_{i}^{\sharp} \\
& =\operatorname{deg}_{Y} \mathscr{M} \otimes \mathscr{N}+\sum_{i}\left(q l \operatorname{deg}\left(\mathscr{M} \widetilde{\otimes} \mathscr{N}, P_{i}\right)+a_{i} m_{i}\right) P_{i}^{\sharp} \\
& =\operatorname{deg}_{Y} \mathscr{M} \otimes \mathscr{N}+\sum_{i} a_{i}+\sum_{i} q l \operatorname{deg}\left(\mathscr{M} \widetilde{\otimes} \mathscr{N}, P_{i}\right) P_{i}^{\sharp} \\
& =q l_{Y}(\mathscr{M} \widetilde{\otimes}) .
\end{aligned}
$$

The last assertion follows from (8.8.5). 
(iii) $T L$ is well defined by $U_{i}\left(m_{i}+z\right)=1+U_{i}(z) \quad(z \in \mathbb{Z})$ for all $i(2.8($ iii $))$. By (8.8.5) and the definition of $R_{i}$ for $\left(X, P_{i}\right) \supset\left(Y, P_{i}\right),(2.8)$ shows that

$$
U_{i}\left(-q l \operatorname{deg}\left(\mathscr{M}, P_{i}\right)\right)=0
$$

for all $i$, whence $T L\left(q l_{Y}(\mathscr{M})\right)=\operatorname{deg}_{Y} \mathscr{M}$. The rest follows from (ii).

(8.10) Definition-Corollary. Let $(X, P) \supset C$ be the germ of a 3-fold terminal singularity of index $m \geq 1$ and a reduced curve, and let $\left(X^{\sharp}, P^{\sharp}\right)$ be the canonical cover. Let $J$ be a $C$-laminal ideal of pure width $d$, and $J^{\sharp}$ the canonical lifting of $J$ at $P$. If an $\ell$-basis $\left\{s_{1}, s_{2}\right\}$ of $I_{C}$ at $P$ satisfies $\left(s_{1}{ }^{d}, s_{2}\right)=J^{\sharp}$ at $P^{\sharp}$, we say that the ordered $\ell$-basis $\left(s_{1}, s_{2}\right)$ is a $(1, d)$-monomializing $\ell$-basis of $I_{C} \supset J$ at $P$, and that $J$ is $(1, d)$-monomializable at $P$ if such $\left(s_{1}, s_{2}\right)$ exists. We note that width $_{J^{\sharp}} s_{1}=1$ and width $_{J^{\sharp}} s_{2}=d$ by (8.3(i)).

Assume that $J$ is $(1, d)$-monomializable at $P$ and let $\mathscr{S}$ be an $\ell$-free

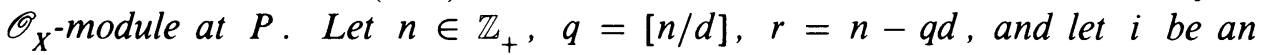
arbitrary integer in $[0, q]$. Then

(i) $\operatorname{gr}^{n}(\mathscr{S}, J), \operatorname{gr}_{C}^{0} \mathscr{S}, \operatorname{gr}_{C}^{1} \mathscr{O}, \operatorname{gr}^{1}(\mathscr{O}, J), \operatorname{gr}^{d}(\mathscr{O}, J), \operatorname{Ker} \beta_{J}$ are $\ell$-free $\mathscr{O}_{C}$-modules at $P$ with their induced $\ell$-structures (8.8.6(ii)), and

$$
\begin{aligned}
& \operatorname{gr}^{n}(\mathscr{S}, J)^{\sharp} \simeq\left(\bigoplus_{i+d j=n} \mathscr{O}_{C^{\sharp}} s_{1}{ }^{i} s_{2}{ }^{j}\right) \otimes_{\mathscr{O}_{C}} \mathscr{S}^{\sharp}, \\
& \left(\operatorname{gr}_{C}^{1} \mathscr{O}\right)^{\sharp}=\mathscr{O}_{C^{\sharp}} s_{1} \oplus \mathscr{O}_{C^{\sharp}} s_{2}, \\
& \operatorname{gr}^{1}(\mathscr{O}, J)^{\sharp}=\mathscr{O}_{C^{\sharp} s_{1}} \quad \text { if } d \geq 2 \text {, } \\
& \operatorname{gr}^{d}(\mathscr{O}, J)^{\sharp}=\mathscr{O}_{C^{\sharp} s_{1}}{ }^{d} \oplus \mathscr{O}_{C^{\sharp}} S_{2}, \\
& \left(\operatorname{Ker} \beta_{J}\right)^{\sharp}= \begin{cases}\mathscr{O}_{C^{\sharp} S_{2}} & \text { if } d \geq 2, \\
0 & \text { if } d=1,\end{cases}
\end{aligned}
$$

(ii) $\gamma^{n}(\mathscr{S}, J)$ induces an $\ell$-isomorphism

$$
\tilde{\gamma}^{n}(\mathscr{S}, J): \widetilde{S}^{q}\left(\operatorname{gr}^{d}(\mathscr{O}, J)\right) \widetilde{\otimes}\left(\operatorname{gr}^{1}(\mathscr{O}, J)\right)^{\tilde{\otimes} r} \tilde{\otimes} \operatorname{gr}_{C}^{0} \mathscr{S} \rightarrow \operatorname{gr}^{n}(\mathscr{S}, J),
$$

(iii) if $d \geq 2$, then we have an isomorphism

$$
\operatorname{gr}^{n, i}(\mathscr{S}, J)^{\sharp} \simeq \mathscr{O}_{C^{\sharp}} S_{1}{ }^{d i+r} s_{2}{ }^{q-i} \otimes_{\mathscr{C}^{\sharp}} \mathscr{S}^{\sharp},
$$

whence $\gamma^{n, i}(\mathscr{S}, J)$ induces an $\ell$-isomorphism

$$
\tilde{\gamma}^{n, i}(\mathscr{S}, J): \operatorname{gr}^{1}(\mathscr{O}, J)^{\widetilde{\otimes}(d i+r)} \widetilde{\otimes}\left(\operatorname{Ker} \beta_{J}\right)^{\widetilde{\otimes}(q-i)} \widetilde{\otimes} \operatorname{gr}_{C}^{0} \mathscr{S} \rightarrow \operatorname{gr}^{n, i}(\mathscr{S}, J) .
$$

Indeed (i) and (ii) are immediate corollaries to (8.3) and (8.8.6) via the definitions in (8.8). For (iii), we also use (8.6).

(8.10.1) Remark. If $m=1$ in $(8.10)$, then $J$ is $(1, d)$-monomializable at $P$ iff $J$ is a nested c.i. at $P$. 
(8.11) Definition-Proposition. Let $(X, P) \supset C$ be the germ of a 3-fold terminal singularity of index $m \geq 1$ and a reduced curve defined by ideal $I_{C}$ and let $\left(X^{\sharp}, P^{\sharp}\right)$ be the canonical cover. Let $C^{\sharp}, I_{C^{\sharp}}$ be the canonical liftings of $C, I_{C}$ at $P$. Let $\left\{s_{1}, s_{2}, e\right\}$ be an $\ell$-basis of $I_{C}$ at $P, b$ and $d$ integers $\geq 2$, and $f \in \mathscr{O}_{X^{\sharp}}$ a semi-invariant such that $\bar{f}=f \bmod I_{C^{\sharp}}$ is a nonzero divisor of $\mathscr{O}_{C^{\sharp}}$. For $n \geq 0$, let

$$
\begin{gathered}
\mathscr{K}_{n}=\sum_{i+b j+d k \geq n}\left(s_{1}{ }^{i} e^{j} s_{2}{ }^{k}\right) \mathscr{O}_{X^{\sharp}} \supset \mathscr{K}_{n}^{\circ}=\sum_{\substack{i+b j+d k \geq n \\
i+j+k \geq 2}}\left(s_{1}{ }^{i} e^{j} s_{2}{ }^{k}\right) \mathscr{O}_{X^{\sharp}}, \\
\mathscr{K}_{n}^{\prime}=\sum_{\substack{i+b j+d k \geq n \\
0 \leq i<b}}\left(s_{1}{ }^{i} e^{j} s_{2}{ }^{k}\right) \mathscr{O}_{X^{\sharp}} .
\end{gathered}
$$

Assume that $f e-s_{1}{ }^{b} \in \mathscr{K}_{b+1}$ at $P^{\sharp}$. Then one has

(i) $J^{\sharp}=\mathscr{K}_{d}$ is a $C^{\sharp}$-laminal ideal of pure width $d$, wt fe $\equiv b$ wt $s_{1} \quad(m)$, width $_{J^{\sharp}} s_{1}=1$, width $J_{J^{\sharp}} s_{2}=d$, width $J_{J^{\sharp}} e=b$, and furthermore for all $n \geq 0$, we have $F^{n}\left(\mathscr{O}_{X^{\sharp}}, J^{\sharp}\right)=\mathscr{K}_{n}=\mathscr{K}_{n}^{\prime}$,

$$
\delta_{J^{\sharp}}^{n}: \operatorname{gr}^{n}\left(\mathscr{O}_{X^{\sharp}}, J^{\sharp}\right) \simeq \bigoplus_{\substack{i+b j+d k=n \\ 0 \leq i<b, 0 \leq j, k}} \mathscr{O}_{C^{\sharp}} s_{1}{ }^{i} e^{j} s_{2}{ }^{k},
$$

and an isomorphism of graded $\mathscr{O}_{C^{\sharp}, P^{\sharp}}$-algebras with $\boldsymbol{\mu}_{m}$-action

$$
\delta_{J^{\sharp}, P^{\sharp}}^{\cdot}: \operatorname{gr}\left(\mathscr{O}_{X^{\sharp}}, J^{\sharp}\right)_{P^{\sharp}} \stackrel{\sim}{\longrightarrow} \mathscr{O}_{C^{\sharp}, P^{\sharp}}\left[S_{1}, S_{2}, E\right] /\left(\bar{f} E-S_{1}^{b}\right),
$$

sending $s_{1}, s_{2}$, e to $S_{1}, S_{2}, E$, respectively. In particular,

$$
\operatorname{Im} \alpha_{J^{\sharp}}=\mathscr{O}_{C^{\sharp}} s_{2}, \quad \operatorname{gr}^{1}\left(\mathscr{O}_{X^{\sharp}}, J^{\sharp}\right)=\mathscr{O}_{C^{\sharp}} s_{1}
$$

(cf. (8.8.6(ii)) for $\alpha_{J^{\sharp}}$ ),

(ii) if we further assume

(a) $d>b$ and $f e-s_{1}{ }^{b}-h s_{2} \in \mathscr{K}_{b+1}^{\circ}$ for some unit $h$ (resp. (b) fe$s_{1}^{b} \in \mathscr{K}_{b+1}^{\circ}$ ), then $\operatorname{gr}_{C^{\sharp}}^{1} \mathscr{O}$ is a free $\mathscr{O}_{C^{\sharp}}$-module with a free basis $\left\{s_{1}, e\right\}$ (resp. $\left.\left\{s_{1}, s_{2}\right\}\right)$ and $\operatorname{Ker} \beta_{J^{\sharp}}$ is an invertible sheaf (cf. (8.8.6(ii))) and

$$
\operatorname{Im} \alpha_{J^{\sharp}}=\bar{f} \cdot \operatorname{Ker} \beta_{J^{\sharp}} \quad\left(\operatorname{resp} . \operatorname{Ker} \beta_{J^{\sharp}}\right),
$$

i.e. $\operatorname{Ker} \beta_{J^{\sharp}}=\mathscr{O}_{C^{\sharp}} e \quad\left(\right.$ resp. $\left.\mathscr{O}_{C^{\sharp}} s_{2}\right)$,

(iii) let $J$ be a $C$-laminal ideal of width $d$ and let $J^{\sharp}=\operatorname{lif} J$. Then $J^{\sharp}=\mathscr{K}_{d}$ iff $s_{2} \in J^{\sharp}$.

If the condition in (iii) holds and if (a) (resp. (b)) in (ii) holds, then we say that the ordered $\ell$-basis $\left(s_{1}, s_{2}, e\right)$ is $a(1, d, b)$-monomializing $\ell$-basis of the first (resp. second) kind of $I_{C} \supset J$ at $P$, and that $f$ is the attached semiinvariant. We say that $J$ is $(1, d, b)$-monomializable at $P$ and $I_{C} \supset J$ at $P$ 
( or simply $P$ ) is of the first kind (resp. the second kind) if such an $\ell$-basis exists.

Proof. (i) Since $s_{1}{ }^{b} \equiv f e \quad\left(\bmod \mathscr{K}_{b+1}\right)$ and $\mathscr{K}_{b+1} \cdot \mathscr{K}_{n-b} \subset \mathscr{K}_{n+1}$, we have a natural surjection

$$
\gamma_{n}: \bigoplus_{\substack{i+b j+d k=n \\ 0 \leq i<b, 0 \leq j, k}} \mathscr{O}_{C^{\sharp}} s_{1}{ }^{i} e^{j} s_{2}{ }^{k} \rightarrow \mathscr{K}_{n} / \mathscr{K}_{n+1}
$$

from a free $\mathscr{O}_{C^{\sharp}}$-module of rank $[n / d]+1$. Let us fix an arbitrary $N \in \mathbb{N}$ for the moment. If $Q^{\sharp} \in C^{\sharp}-\left\{P^{\sharp}\right\}$ is a point close to $P^{\sharp}$ such that $f\left(Q^{\sharp}\right) \neq 0$, then $e \in \sum_{i+d k \geq b}\left(s_{1}{ }^{i} s_{2}{ }^{k}\right)$ by Nakayama's lemma. Thus $I_{C^{\sharp}, Q^{\sharp}}=\left(s_{1}, s_{2}\right)$ and $\mathscr{K}_{n, Q^{\sharp}}=\sum_{i+d k \geq n}\left(s_{1}{ }^{i} s_{2}{ }^{k}\right)$. By (8.3(i)), $\mathscr{K}_{n} / \mathscr{K}_{n+1}$ is free of rank $[n / d]+1$ at $Q^{\sharp}, \mathscr{K}_{d}$ is a $C^{\sharp}$-laminal ideal of pure width $d$ at $Q^{\sharp}$, and $\mathscr{K}_{n}=F^{n}\left(\mathscr{O}_{X^{\sharp}}, \mathscr{K}_{d}\right)$ for all $n \in[0, N]$ if $Q^{\sharp}$ is close enough to $P^{\sharp}$. Hence $\left(\operatorname{Ker} \gamma_{n}\right)_{Q^{\sharp}}=0$, and $\operatorname{Ker} \gamma_{n}=0$ by $\operatorname{Ker} \gamma_{n} \subset \operatorname{Sat}(0)=0(n \in[0, N])$. Hence $\gamma_{n}$ is an isomorphism for all $n \in[0, N]$ and we note $\sqrt{\mathscr{K}}_{n}=I_{C^{\sharp}}$ for all $n \geq 1$. Hence $\mathscr{O}_{X^{\sharp}} / \mathscr{K}_{n}$ has no embedded primes for all $n \in[0, N]$, whence $\mathscr{K}_{d}=\operatorname{Sat}\left(\mathscr{K}_{d}\right) \quad\left(=J^{\sharp}\right)$ is a $C^{\sharp}$-laminal ideal of pure width $d$ and $\mathscr{K}_{n}=\operatorname{Sat}\left(\mathscr{K}_{n}\right)=F^{n}\left(\mathscr{O}_{X^{\sharp}}, J^{\sharp}\right)$ for all $n \in$ $[0, N]$ because $\left(\mathscr{K}_{n}\right)_{Q^{\sharp}}=F^{n}\left(\mathscr{O}_{X^{\sharp}}, \mathscr{K}_{d}\right)_{Q^{\sharp}}$ at nearby points $Q^{\sharp}\left(\neq P^{\sharp}\right)$. Hence it holds for all $n \geq 0$ and $\delta_{J^{\sharp}}^{n}$ is a $\boldsymbol{\mu}_{m}$-isomorphism for all $n \geq 0$, because $N$ was chosen arbitrarily. Hence $\mathscr{K}_{n}=\mathscr{K}_{n+1}+\mathscr{K}_{n}^{\prime}$ for all $n>0$, and $\mathscr{K}_{n}=\mathscr{K}_{n+n^{\prime}}+\mathscr{K}_{n}^{\prime}$ for all $n, n^{\prime}>0$. If $n^{\prime}$ is large enough, then $\mathscr{K}_{n+n^{\prime}} \subset I_{C} \mathscr{K}_{n}$ and $\mathscr{K}_{n}=\mathscr{K}_{n}^{\prime}$ by Nakayama's lemma. By $\delta_{J^{\sharp}}^{b}\left(s_{1}^{b}\right)=\bar{f} \delta_{J^{\sharp}}^{b}(e)$, we have wt $f e \equiv b$ wt $s_{1} \quad(m)$. Since $d \geq 2$ and $\operatorname{gr}_{C^{\sharp}}^{0} J^{\sharp}=\operatorname{gr}^{d}\left(\mathscr{O}, J^{\sharp}\right)$ has a free basis $\left\{s_{2}, s_{1}{ }^{d-b[d / b]} \cdot e^{[d / b]}\right\}$ by $\delta_{J^{\sharp}}^{d}$, we see that $\operatorname{Im} \alpha_{J^{\sharp}}=\mathscr{O}_{C^{\sharp}} s_{2}$ if $d \neq b$ by $d-b[d / b]+[d / b] \geq 2$. If $d=b$, then $f e-s_{1}{ }^{b} \in \mathscr{K}_{b+1}$ implies $e \in I^{(2)}$ by $b \geq 2$, whence $\alpha_{J^{\sharp}}(e)=0$ and $\operatorname{Im} \alpha_{J^{\sharp}}=\mathscr{O}_{C^{\sharp}} s_{2}$ anyway. The rest is obvious.

(ii) The assertion on the free basis is straightforward. By (8.4), $\operatorname{Ker} \beta_{J^{\sharp}}=$ $\operatorname{Sat}\left(\operatorname{Im} \alpha_{J^{\sharp}}\right)$ and (ii) follows from (i).

(iii) The only-if part is obvious by definition. By (8.8.6), $J^{\sharp}$ is a $C^{\sharp}$-laminal ideal of width $d$. Assume that $s_{2} \in J^{\sharp}$. Let $Q^{\sharp} \in C^{\sharp}-\left\{P^{\sharp}\right\}$ be a point close to $P^{\sharp}$ such that $f\left(Q^{\sharp}\right) \neq 0$. Then one has $I_{C^{\sharp}, Q^{\sharp}}=\left(s_{1}, s_{2}\right)$, and the same argument as in the proof of $(8.3(\mathrm{ii}))$ can be used to get $J_{Q^{\sharp}}^{\sharp}=\left(\mathscr{K}_{d}\right)_{Q^{\sharp}}$. Then $J^{\sharp}=\operatorname{Sat}\left(J^{\sharp}\right)=\operatorname{Sat}\left(\mathscr{K}_{d}\right)=\mathscr{K}_{d}$.

(8.11.1) Remark. Let the notation and assumptions be as in (8.11(i)). Let $\mathscr{S}$ be a locally $\ell$-free $\mathscr{O}_{X}$-module and $J=$ quo $\left(J^{\sharp}\right)$ (8.8.1). Then

(i) if $\bar{f}$ is a unit then $\left(s_{1}, s_{2}\right)$ is a $(1, d)$-monomializing $\ell$-basis of $I_{C} \supset J$ at $P$, 
(ii) using (8.6) and (8.8.6), one sees that $\operatorname{gr}^{n}(\mathscr{S}, J), \operatorname{gr}_{C}^{0} \mathscr{S}, \mathscr{M}=\operatorname{gr}^{1}(\mathscr{O}, J)$, $\operatorname{gr}^{d}(\mathscr{O}, J), \mathscr{L}=\operatorname{Ker} \beta_{J}, \operatorname{gr}^{n, i}(\mathscr{S}, J)$ are $\ell$-free $\mathscr{O}_{C}$-modules at $P$ with the descriptions

$$
\begin{aligned}
& \mathscr{L}^{\sharp}= \begin{cases}\mathscr{O}_{C^{\sharp}} e & (P \text { first kind }), \\
\mathscr{O}_{C^{\sharp}} s_{2} & (P \text { second kind }),\end{cases} \\
& \mathscr{M}^{\sharp}=\mathscr{O}_{C^{\sharp}} s_{1}, \\
& \operatorname{gr}^{d}(\mathscr{O}, J)^{\sharp}=\mathscr{O}_{C^{\sharp}} s_{2} \oplus \mathscr{O}_{C^{\sharp}} e^{[d / b]} s_{1}{ }^{d-b[d / b]} \text {, } \\
& \operatorname{gr}^{n, i}(\mathscr{O}, J)^{\sharp}=\mathscr{O}_{C^{\sharp}} S_{2}{ }^{q-i} e^{[(d i+r) / b]} s_{1}{ }^{(d i+r)-b[(d i+r) / b]},
\end{aligned}
$$

and that $\gamma^{n, i}(\mathscr{S}, J) \quad(n \geq 0)$ induces an $\ell$-isomorphism

$$
\tilde{\gamma}^{n, i}(\mathscr{S}, J): \mathscr{M}^{\tilde{\otimes}(d i+r)} \widetilde{\otimes} \mathscr{D}_{P}^{\tilde{\otimes} e_{j}} \widetilde{\otimes} \mathscr{L}^{\widetilde{\otimes}(q-i)} \widetilde{\otimes} \operatorname{gr}_{C}^{0} \mathscr{S} \rightarrow \operatorname{gr}^{n, i}(\mathscr{S}, J)
$$

for $i \in[0, q]$, where $q=[n / d]$ and $r=n-q d, e_{j}=[(d i+r) / b]-(q-i)$ (resp. $e_{j}=[(d i+r) / b]$ ) if $P$ is of the first (resp. second) kind, and $\mathscr{D}_{P}$ is an $\ell$-invertible $\mathscr{O}_{C}$-module at $P$ given by

$$
\mathscr{D}_{P}=\left(\mathscr{O}_{C^{\sharp}} \bar{f}^{-1}\right)^{\mu_{m}} \subset \mathscr{D}_{P}^{\sharp}=\mathscr{O}_{C} \bar{f}^{-1} .
$$

This is because $s_{1}, s_{2}$ are $\ell$-free $\ell$-bases of $\mathscr{M}, \mathscr{L} \widetilde{\otimes} \mathscr{D}^{\tilde{\otimes}(-1)}$ (resp. $\mathscr{M}, \mathscr{L}$ ), and $s_{1}{ }^{d i+r} \equiv(f \cdot e)^{[(d i+r) / b]} \cdot s_{1}{ }^{d i+r-b[(d i+r) / b]}$, and

(iii) if there is a global curve $C$ extending $(C, P)$ (cf. (8.9)), one can trivially extend $\mathscr{D}_{P}$ to an $\ell$-invertible $\mathscr{O}_{C}$-module $\mathscr{D}$ on global $C$ so that the unit section 1 of $\mathscr{D}_{P}$ extends to a global section of $\mathscr{D}$ and generates $\mathscr{D}$ outside $P$. By construction, one sees $l \operatorname{deg}_{C} \mathscr{D}=m^{-1} \operatorname{len}_{P}\left(\mathscr{O}_{C} /\left(\bar{f}^{m}\right) \mathscr{O}_{C}\right)$.

(8.12) Theorem. Let $X \supset C \simeq \mathbb{P}^{1}$ be an extremal nbd and let $J$ be a $C$-laminal ideal of pure width $d$ and let $P_{1}, \ldots, P_{t} \in C$ be such that $X$ is smooth and $J$ is a nested c.i. at arbitrary points of $C-\left\{P_{1}, \ldots, P_{t}\right\}$. We assume that there exists $s \in[0, d](s=0$ if $d=1)$ such that $J$ is $\left(1, d, b_{j}\right)$-monomializable for some $b_{j}$ (resp. $(1, d)$-monomializable) at $P_{j}$ for all $j \leq s$ (resp. $j \geq s+1)$. We also assume that there exists $s^{\prime} \in[0, s]$ such that $P_{j}$ is of the first (resp. second) kind if $j \in\left[1, s^{\prime}\right]$ (resp. $\left.\left[s^{\prime}+1, s\right]\right)$. For each $j \leq s$, let $\mathscr{D}_{j}$ be the $\ell$-invertible $\mathscr{O}_{C}$-module obtained as $\mathscr{D}$ at $P_{j}$

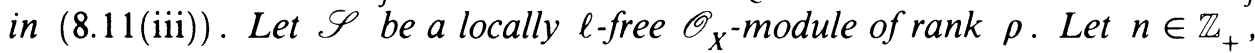
$q=[n / d], r=n-q d$, and let $i$ be an arbitrary integer in $[0, q]$. Then $\operatorname{gr}^{n}(\mathscr{S}, J), \operatorname{gr}_{C}^{0} \mathscr{S}, \operatorname{gr}^{d}(\mathscr{O}, J)$ are locally $\ell$-free $\mathscr{O}_{C}$-modules of rank $q \rho+\rho$, $\rho, 2$, respectively, and

(i) if $d=1$, then $J=I_{C}, \operatorname{gr}^{n}(\mathscr{S}, J)=\operatorname{gr}_{C}^{n} \mathscr{S}, \operatorname{gr}^{1}(\mathscr{O}, J)=\operatorname{gr}_{C}^{1} \mathscr{O}$, and $\gamma^{n}\left(\mathscr{S}, I_{C}\right)$ induces an $\ell$-isomorphism

$$
\tilde{\gamma}^{n}\left(\mathscr{S}, I_{C}\right): \tilde{S}^{n}\left(\mathrm{gr}_{C}^{1} \mathscr{O}\right) \widetilde{\otimes} \operatorname{gr}_{C}^{0} \mathscr{S} \stackrel{\sim}{\longrightarrow} \operatorname{gr}_{C}^{n} \mathscr{S}
$$


and one has $l \operatorname{deg}_{C} \operatorname{gr}_{C}^{1} \mathscr{O} \geq 0$, and

(ii) if $d \geq 2$, then $\mathscr{M}=\operatorname{gr}^{1}(\mathscr{O}, J), \mathscr{L}=\operatorname{Ker} \beta_{J}$ (8.2.2), and $\mathrm{gr}^{n, i}(\mathscr{S}, J)$ (8.6) are $\ell$-invertible $\mathscr{O}_{C}$-modules, $\beta_{J}$ induces an $\ell$-exact sequence

$$
0 \rightarrow \mathscr{L} \rightarrow \mathrm{gr}_{C}^{1} \mathscr{O} \rightarrow \mathscr{M} \rightarrow 0,
$$

and $\gamma^{n, i}(\mathscr{S}, J)$ (8.6) induces an l-isomorphism

$$
\tilde{\gamma}^{n, i}(\mathscr{S}, J): \mathscr{M}^{\widetilde{\otimes}(d i+r)} \tilde{\otimes}\left(\widehat{\bigotimes}_{j=1}^{s} \mathscr{D}_{j}^{\widetilde{\otimes} e_{j}}\right) \tilde{\otimes} \mathscr{L}^{\widetilde{\otimes}(q-i)} \tilde{\otimes} \mathrm{gr}_{C}^{0} \mathscr{S} \rightarrow \mathrm{gr}^{n, i}(\mathscr{S}, J),
$$

where $e_{j}=\left[(d i+r) / b_{j}\right]-(q-i) \quad\left(\right.$ resp. $\left.\left[(d i+r) / b_{j}\right]\right)$ for $j \in\left[1, s^{\prime}\right] \quad$ (resp. $\left.j \in\left[s^{\prime}+1, s\right]\right)$, and one has

$$
l \operatorname{deg}_{C} \mathscr{M}+\frac{1}{d} l \operatorname{deg}_{C} \mathscr{L}+\sum\left(\frac{1}{b_{j}}-\frac{1}{d}\right) l \operatorname{deg}_{C} \mathscr{D}_{j}+\sum^{\prime} \frac{1}{b_{j}} l \operatorname{deg}_{C} \mathscr{D}_{j} \geq 0,
$$

where $\sum$ (resp. $\left.\sum^{\prime}\right)$ is over $P_{j}$ of the first (resp. second) kind.

Proof. The assertions follow mostly from (8.10), (8.11), (8.11.1). For (i), we only need to show $l \operatorname{deg}_{C} \operatorname{gr}_{C}^{1} \mathscr{O} \geq 0$. By (8.9.1) and $\tilde{\gamma}^{n}\left(\mathscr{O}_{X}, I_{C}\right)$, one has

$$
\operatorname{deg}_{C} \operatorname{gr}_{C}^{n} \mathscr{O} \leq l \operatorname{deg}_{C} \operatorname{gr}_{C}^{n} \mathscr{O}=\frac{1}{2} n(n+1) l \operatorname{deg}_{C} \operatorname{gr}_{C}^{1} \mathscr{O} \text {. }
$$

Thus $l \operatorname{deg}_{C} \operatorname{gr}_{C}^{1} \mathscr{O} \geq 0$ by (8.7). For (ii), we need to prove the last inequality. We note that $l \operatorname{deg}_{C} \mathscr{D}_{j} \geq \operatorname{deg}_{C} \mathscr{D}_{j} \geq 0$ (8.9.1) since $\mathscr{D}_{j}$ has a nonzero global section (8.11(iii)). Thus by (8.9.1) and $\widetilde{\gamma}^{n, i}\left(\mathscr{O}_{X}, J\right)$, one has

$$
\begin{aligned}
\operatorname{deg}_{C} \operatorname{gr}^{n, i}\left(\mathscr{O}_{X}, J\right) \leq & l \operatorname{deg}_{C} \operatorname{gr}^{n, i}\left(\mathscr{O}_{X}, J\right) \\
\leq & (d i+r) l \operatorname{deg}_{C} \mathscr{M}+(q-i) l \operatorname{deg}_{C} \mathscr{L} \\
& +\sum_{j=1}^{s} \frac{d i+r}{b_{j}} l \operatorname{deg}_{C} \mathscr{D}_{j}-\sum_{j=1}^{s^{\prime}}(q-i) l \operatorname{deg}_{C} \mathscr{D}_{j},
\end{aligned}
$$

and summing these up for $i=0, \ldots, q(=[n / d])$, one has

$$
\begin{aligned}
& \operatorname{deg}_{C} \operatorname{gr}^{n}(\mathscr{O}, J) \\
& \leq \frac{n^{2}}{2 d}\left(l \operatorname{deg}_{C} \mathscr{M}+\frac{1}{d} l \operatorname{deg}_{C} \mathscr{L}+\sum_{j=1}^{s} \frac{1}{b_{j}} l \operatorname{deg}_{C} \mathscr{D}_{j}-\sum_{j=1}^{s^{\prime}} \frac{1}{d} l \operatorname{deg}_{C} \mathscr{D}_{j}\right)+O(n),
\end{aligned}
$$

where $O(n)$ is a term bounded by (constant) $\cdot n$ as $n \rightarrow \infty$. Thus again by (8.7), we are done.

(8.13) Let $X$ be an analytic 3-fold with only terminal singularities and $C$ a smooth curve in $X$ defined by ideal $I$. Let $J$ be a $C$-laminal ideal of width $d \geq 2$. Let

$$
0 \rightarrow \mathrm{gr}^{d, 1}(\mathscr{O}, J) \rightarrow \mathrm{gr}_{C}^{0} J \rightarrow \mathrm{gr}^{d, 0}(\mathscr{O}, J) \rightarrow 0
$$

be the $\ell$-exact sequence given in (8.6). 
Assume that it has a splitting

$$
\operatorname{gr}_{C}^{0} J=\operatorname{gr}^{d, 1}(\mathscr{O}, J) \oplus \mathscr{L}_{J}
$$

into invertible sheaves on $C$, where $\mathscr{L}_{J}$ is a subbundle of $\operatorname{gr}_{C}^{0} J$ of rank 1 and hence $\mathscr{L}_{J}$ has an induced $\ell$-structure as a $\operatorname{gr}_{C}^{0} J$-saturated subsheaf of $\operatorname{gr}_{C}^{0} J$ (8.8.1). We note that (8.13.1) is not necessarily an $\ell$-splitting. Let $K$ be a $C$-primary ideal of $\mathscr{O}_{X}$ such that $J \supset K \supset F^{1} J$ and $K / F^{1} J=\mathscr{L}_{J}$. Let $P$ be a point on $C,\left(X^{\sharp}, P^{\sharp}\right)$ the canonical cover of germ $(X, P)$, and $C^{\sharp} \subset\left(X^{\sharp}, P^{\sharp}\right)$ the canonical lifting of $C$. Then we can study $K$ in the following four cases.

(8.14) Proposition. Assume that $J$ is a nested c.i. at $P$ (of width $d \geq 2$ as above). Then $K$ is a nested c.i. at $P$ of width $d+1$ such that $F^{i}(\mathscr{O}, K)_{P}=$ $F^{i}(\mathscr{O}, J)_{P}$ for $i \leq d$. Thus $K$ is a $C$-laminal ideal of width $d+1$ such that $F^{i}(\mathscr{O}, K)=F^{i}(\mathscr{O}, J)$ for $i \leq d$, whence $\beta_{K}=\beta_{J}$ and $\operatorname{Ker} \beta_{K}=\operatorname{Ker} \beta_{J}$.

Proof. The ideal $I$ has a basis $\{u, v\}$ at $P$ such that $J_{P}=\left(u^{d}, v\right)$. Then $\mathscr{L}_{J}$ is generated by $w=v+\alpha u^{d}$ for some $\alpha \in \mathscr{O}_{X, P}$ by $(8.3(\mathrm{i}))$, whence $I_{P}=(u, w), J_{P}=\left(u^{d}, w\right)$, and $K_{P}=\left(u^{d+1}, w\right)(8.3(\mathrm{ii}))$, and the equality holds by $(8.3(\mathrm{i}))$. Thus $K_{P}$ is a $C$-laminal ideal of width $d+1$. Since $d \geq 2$, $\operatorname{gr}^{1}(\mathscr{O}, J)=\operatorname{gr}^{1}(\mathscr{O}, K)=(u, v) /\left(u^{2}, v\right)$, whence $\beta_{J}=\beta_{K}: \operatorname{gr}_{C}^{1} \mathscr{O} \rightarrow \operatorname{gr}^{1}(\mathscr{O}, J)$ because they are naturally induced maps (8.2.2).

Learning that $K$ is a $C$-laminal ideal of width $d+1$, we see

(8.14.1) Corollary. $F^{1} J / F^{1} K=\mathrm{gr}^{d+1,1}(\mathscr{O}, K)$ and the natural map $K \rightarrow J$ induces an $\ell$-isomorphism $\mathrm{gr}^{d+1,0}(\mathscr{O}, K)=K / F^{1} \mathrm{~J} \stackrel{\sim}{\longrightarrow} \mathscr{L}_{J}$.

Proof. Since $\operatorname{gr}^{d+1,1}(\mathscr{O}, K)$ is a $\operatorname{gr}_{C}^{0} K$-saturation of $\left(I^{d+1}+F^{1} K\right) / F^{1} K$ in $\operatorname{gr}_{C}^{0} K$ via $\gamma^{d+1,1}(\mathscr{O}, K)(8.6(\mathrm{ii}))$ and since $I^{d+1} \subset F^{1} J$ by width $J=d$, we see $\operatorname{gr}^{d+1,1}(\mathscr{O}, K) \subset F^{1} J / F^{1} K$, which are both $\operatorname{gr}_{C}^{0} K$-saturated submodules of $\mathrm{gr}_{C}^{0} K$ of rank 1. Hence $\mathrm{gr}^{d+1,1}(\mathscr{O}, K)=F^{1} J / F^{1} K$. Since $J, K$, $F^{1} J$ are $\mathscr{O}_{X}$-saturated submodules of $\mathscr{O}_{X}$ and since $\mathscr{L}_{J}=K+F^{1} J / F^{1} J$ is a $J / F^{1} J$-saturated submodule of $J / F^{1} J$, the two expressions $K / F^{1} J$ and $K+$ $F^{1} J / F^{1} J$ induce the same $\ell$-structure on $\mathscr{L}_{J}$ by $(8.8 .1)$.

(8.15) Proposition. Assume that $\left(C^{\sharp}, P^{\sharp}\right)$ is smooth and that $I \supset J$ has a $(1, d)$-monomializing $\ell$-basis $\left(s_{1}, s_{2}\right)$ at $P$. There exists a semi-invariant $\alpha \in$ $\mathscr{O}_{X^{\sharp}, P \sharp}$ such that wt $\alpha \equiv \mathrm{wt} s_{2}-d \mathrm{wt} s_{1}$, or $-\mathrm{wt} s_{2}+d \mathrm{wt} s_{1}(\bmod m)$ and such that

(8.15.1) if $\operatorname{gr}_{C}^{0} J=\operatorname{gr}^{d, 1}(\mathscr{O}, J) \oplus \mathscr{L}_{J}$ (8.13.1) is an $\ell$-splitting at $P$, then $\left(s_{1}, s_{2}+\alpha s_{1}{ }^{d}\right)$ is a $(1, d+1)$-monomializing $\ell$-basis for $I \supset K$ at $P$, and 
(8.15.2) if $\operatorname{gr}_{C}^{0} J=\operatorname{gr}^{d, 1}(\mathscr{O}, J) \oplus \mathscr{L}_{J}$ (8.13.1) is not an $\ell$-splitting at $P$, then $0<\operatorname{ord} \alpha<\infty$, and $\left(s_{1}, \alpha s_{2}+s_{1}{ }^{d}, s_{2}\right)$ is a $(1, d+1, d)$-monomializing $\ell$-basis of the first kind for $I \supset K$ at $P$ with attached semi-invariant $-\alpha$ and relation:

$$
(-\alpha) s_{2}-s_{1}^{d}=-\left(s_{2}+\alpha s_{1}^{d}\right)
$$

Proof. Since $C^{\sharp}$ is smooth, we see that $\operatorname{lif}\left(\mathscr{L}_{J}\right)=\mathscr{O}_{C^{\sharp}}\left(\bar{\beta} s_{1}+\bar{\gamma} s_{1}{ }^{d}\right)$ for some semi-invariants $\bar{\beta}(\neq 0), \bar{\gamma} \in \mathscr{O}_{C^{\sharp}}$ by $(8.10($ iii $))$, and that $\mathscr{L}_{J}$ defines an $\ell$-splitting iff ord $\bar{\beta} \leq$ ord $\bar{\gamma}$. In case of (8.15.1) (resp. (8.15.2)), we take a semiinvariant representative $\alpha \in \mathscr{O}_{X^{\sharp}, P^{\sharp}}$ of $\bar{\gamma} \cdot \bar{\beta}^{-1}$ (resp. $\bar{\beta} \cdot \bar{\gamma}^{-1}$ ). Since $s_{2}+\alpha s_{1}{ }^{d}$ (resp. $\alpha s_{2}+s_{1}{ }^{d}$ ) belongs to $K,(8.10)($ resp. (8.11)) applies.

(8.16) Proposition. Assume that $\left(C^{\sharp}, P^{\sharp}\right)$ is smooth, that $I \supset J$ has a $(1, d, b)$-monomializing $\ell$-basis $\left(s_{1}, s_{2}, e\right)$ at $P$ with attached semi-invariant $f$, and that $\operatorname{gr}_{C}^{0} J=\operatorname{gr}^{d, 1}(\mathscr{O}, J) \oplus \mathscr{L}_{J}(8.13 .1)$ is an $\ell$-splitting at $P$. Let $q=[d / b]$ and $r=d-q b$. Assume that $I \supset J$ is of the first (resp. second) kind at $P$.

Then there exists a semi-invariant $\alpha \in \mathscr{O}_{X^{\sharp}, P \sharp}$ such that wt $\alpha \equiv \mathrm{wt} s_{2}$ $r$ wt $s_{1}-q$ wt $e(\bmod m)$ and $\left(s_{1}, s_{2}+\alpha s_{1}{ }^{r} e^{q}, e\right)$ is a $(1, d+1, b)$-monomializing $\ell$-basis of the first (resp. second) kind for $I \supset K$ at $P$ with attached semi-invariant $f$.

Proof. By $(8.11(\mathrm{i}))$, there exists $\bar{\alpha} \in \mathscr{O}_{C^{\sharp}}$ such that $\mathscr{L}_{J}=\mathscr{O}_{C^{\sharp}}\left(s_{2}+\bar{\alpha} s_{1}^{r} e^{q}\right)$. Let $s_{3}=s_{2}+\alpha s_{1}{ }^{r} e^{q}$ for some semi-invariant representative $\alpha$ of $\bar{\alpha}$, whence $s_{3} \in K$. Let $f e-s_{1}^{b}=g\left(s_{1}, s_{2}, e\right)$ be the relation given in $(8.11(\mathrm{ii}))$. Then $f e-s_{1}{ }^{b}=g\left(s_{1}, s_{3}-\alpha s_{1}{ }^{r} e^{q}, e\right)$ is the relation for a $(1, d+1, b)$-monomializing $\ell$-basis $\left(s_{1}, s_{3}, e\right)$. If $\left(s_{1}, s_{2}, e\right)$ is of the first kind, then $q+r \geq 2$ by $d>b$, whence $\left(s_{1}, s_{3}, e\right)$ is of the first kind. If $\left(s_{1}, s_{2}, e\right)$ is of the second kind, then $g\left(s_{1}, s_{2}, e\right) \in\left(s_{1}, s_{2}, e\right)^{2}$ and $g\left(s_{1}, s_{3}-\alpha s_{1}{ }^{r} e^{q}, e\right) \in\left(s_{1}, s_{3}, e\right)^{2}$. The rest is similar.

\section{EXISTENCE OF "GOOD" ANTICANONICAL DiViSOR (DELICATE CASE).}

The main purpose of this section is to treat the delicate case (6.7.4), and our main result is $(9.10)$.

Unless otherwise mentioned, we will work under the notation and assumptions of the following (9.1) throughout this section.

(9.1) Let $X \supset C \simeq \mathbb{P}^{1}$ be an extremal nbd with 2 points $P, P^{\prime}$ of indices $m, m^{\prime} \geq 3$. Then by (6.7), this is case (6.7.4);

(*) $\quad X \supset C$ has no other singular points, and $P, P^{\prime}$ are of size 1 and of type (IA), (IA ${ }^{\vee}$ ), or (IIA). 
Since $X \supset C$ has only one imprimitive point (1.13), we will assume that $P^{\prime}$ is a primitive point. Let $\bar{m}$ and $s$ be the subindex and the splitting degree of $P$. We note that $m=s \bar{m}$, and that $s=1$ iff $P$ is primitive.

Let $I \subset \mathscr{O}_{X}$ be the sheaf of defining ideals of $C$ in $X$. Let $\pi^{\sharp}:\left(X^{\sharp}, P^{\sharp}\right) \rightarrow$ $(X, P)$ (resp. $\left.\pi^{b}:\left(X^{b}, P^{b}\right) \rightarrow\left(X, P^{\prime}\right)\right)$ be the canonical cover and $\left(C^{\sharp}, P^{\sharp}\right)=$ $\pi^{-1}(C)_{\text {red }}\left(\operatorname{resp} . \quad\left(C^{b}, P^{b}\right)=\pi^{b^{-1}}(C)_{\text {red }}\right)$. Let $I^{\sharp}$ (resp. $I^{b}$ ) be the canonical lifting of $I$ at $P$ (resp. $P^{\prime}$ ). Let $x=\left(x_{1}, \ldots, x_{4}\right)$ and $t$ (resp. $x^{\prime}=$ $\left(x_{1}^{\prime}, \ldots, x_{4}^{\prime}\right)$ and $\left.t^{\prime}\right)$ be normalized $\ell$-coordinates $(2.6)$ at $P$ (resp. $P^{\prime}$ ) such that $a_{i}=C^{\sharp}$-ord $x_{i}$ (resp. $a_{i}^{\prime}=C^{b}$-ord $x_{i}^{\prime}$ ) with $i \in[1,4]$ as in $(4.2)$. We will use ord $=C^{\sharp}$-ord, wt $=C^{\sharp}$-wt, ow $=C^{\sharp}$-ow, $R, U$ for $X \supset C$ at $P$ (resp. $\operatorname{ord}^{\prime}=C^{b}$-ord, $\mathrm{wt}^{\prime}=C^{b}$-wt, ow ${ }^{\prime}=C^{b}$-ow, $R^{\prime}, U^{\prime}$ for $X \supset C$ at $\left.P^{\prime}\right)$ in the sense introduced in (2.5) and (2.8).

We note that if $P$ (resp. $P^{\prime}$ ) is of type (IA) and $a_{2}=1$ (resp. $a_{2}^{\prime}=1$ ), then one may permute $x_{1}$ and $x_{3}$ (resp. $x_{1}^{\prime}$ and $x_{3}^{\prime}$ ) (cf. (4.2)).

We make a preliminary observation at $P$, which applies also to $P^{\prime}$ modulo obvious changes.

(9.1.1) Lemma. Under the above notation and assumptions, assume that $\left(C^{\sharp}, P^{\sharp}\right)$ is smooth (resp. $P$ is ordinary (4.5)). Let $\mathscr{E}$ be a torsion-free $\mathscr{O}_{C}$-module of rank 2 with $\ell$-structure (resp. $\left.\mathscr{E}=\mathrm{gr}_{C}^{0} I\right)$. Then

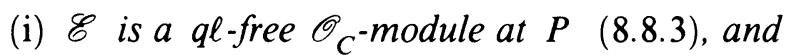

(ii) for an arbitrary splitting $\mathscr{E}=\mathscr{O}_{C} s_{1} \oplus \mathscr{O}_{C} s_{2}$ near $P$, there exists $\mu \in \mathbb{C}$ with the following property.

For an arbitrary $s_{3} \in \mathscr{E} \otimes \mathscr{O}_{C, P}$ with $s_{3}(P)=\mu s_{1}(P)+s_{2}(P) \quad(\in \mathscr{E} \otimes \mathbb{C}(P))$, there exists an $\ell$-free $\ell$-basis $\left\{u_{1}, u_{2}\right\}$ of $\mathscr{E}$ at $P$ such that $s_{1} \in \mathscr{O}_{C^{\sharp}, P^{\sharp}} u_{1}$ and $s_{3} \in \mathscr{O}_{C^{\sharp}, P \sharp} u_{2}$.

In particular, $\mathscr{O}_{C} s_{1}$ is an $\ell$-invertible $\mathscr{O}_{C}$-module at $P$ (with its induced $\ell$-structure (8.8.1)) with an $\ell$-free $\ell$-basis $u_{1}$ at $P$, and $\mathscr{E}=\mathscr{O}_{C} s_{1} \widetilde{\oplus} \mathscr{O}{ }_{C} s_{3}$ is an $\ell$-splitting at $P$.

Proof. (i) is obvious because $\mathscr{E}^{\sharp}$ is $\mathscr{O}_{C^{\sharp}}$-free of rank 2 (8.8.6) by the assumption. Let $\left\{v_{1}, v_{2}\right\}$ be an $\ell$-free $\ell$-basis of $\mathscr{E}$ at $P$ such that $s_{i}=f_{i 1} v_{1}+f_{i 2} v_{2}$ $(i=1,2)$ and ord $f_{11} \leq$ ord $f_{12}$, where $f_{i j}$ are semi-invariants in $\mathscr{O}_{C^{\sharp}, P^{\sharp}}$. Then we have

(9.1.2) Claim. $R\left(\right.$ wt $\left.v_{1}\right), R\left(\right.$ wt $\left.v_{2}\right)<\bar{m}$, and if

$$
(z, w) \in \pm\left(C^{\sharp}-\mathrm{wt}\left(v_{1}\right)-C^{\sharp}-\mathrm{wt}^{*}\left(v_{2}\right)\right)+\mathbb{Z}_{+}(\bar{m}, 0) \subset \mathbb{Z} \times \mathbb{Z}_{m}
$$

satisfies $z \geq 0$ (cf. $(2.8))$, then $(z, w) \in$ ow $\left(C^{\sharp}\right)$.

Indeed, if $\left(C^{\sharp}, P^{\sharp}\right)$ is smooth then (9.1.2) follows from ow $\left(C^{\sharp}\right) \simeq \mathbb{Z}_{+}$(2.5). If $P$ is ordinary, then one sees that $P$ is of type (IA) or $\left(I^{\vee}\right)$ and that $\operatorname{siz}_{P}=1$ by $(*)$ in $(9.1)$, where the claim follows from (4.5.2). 
By the choice of $f_{i j}$, we see that

(9.1.3) ow $f_{i j} \in C^{\sharp}$-ow ${ }^{*}\left(v_{j}\right)+\mathbb{Z}_{+}(\bar{m}, 0)$ for all $i, j(2.8(\mathrm{i}))$,

(9.1.4) for each $i \in[1,2]$, ow $f_{i j}=C^{\sharp}$-ow $^{*}\left(v_{j}\right)$ for some $j$.

This is because $\mathscr{O}_{C} s_{i}$ is an $\mathscr{E}$-saturated submodule of $\mathscr{E}$. Then we will prove

(9.1.5) Claim. ow $f_{21}-$ ow $f_{11} \in \mathbb{Z}_{+}(\bar{m}, 0)$, and if ord $f_{21}>$ ord $f_{11}$, then $u_{1}=s_{1} / f_{11}$ and $u_{2}=s_{2} / f_{22}$ form an $\ell$-free $\ell$-basis for $\mathscr{E}$ at $P$.

Proof of Claim. Since ord $f_{11} \leq$ ord $f_{12}$ by the choice of $v_{j}$, we have ord $f_{11}=$ $R\left(\right.$ wt $\left.v_{1}\right)$ or ord $f_{11} \leq$ ord $f_{12}=R\left(\right.$ wt $\left.v_{2}\right)$ by (9.1.4), whence ord $f_{11}<\bar{m}$ in either case by (9.1.2), and ow $f_{11}=C^{\sharp}$-ow $^{*}\left(v_{1}\right)$ by (9.1.3). Thus ow $f_{21}-$ ow $f_{11} \in \mathbb{Z}_{+}(\bar{m}, 0) \subset$ ow $\left(C^{\sharp}\right)$ as claimed. Assume now ord $f_{21}>$ ord $f_{11}$ as in (9.1.5). Then ord $f_{21} \geq \bar{m}+R\left(\right.$ wt $\left.v_{1}\right)$ (9.1.3), whence ow $f_{22}=C^{\sharp}$-ow ${ }^{*}\left(v_{2}\right)$ by (9.1.4). Thus ord $f_{12}-$ ord $f_{11}=$ ord $f_{12}-R\left(\mathrm{wt} v_{1}\right) \geq 0$ and ord $f_{21}-\operatorname{ord} f_{22}=$ ord $f_{21}-R\left(\right.$ wt $\left.v_{2}\right)>0$ by $R\left(\right.$ wt $\left.v_{2}\right)<\bar{m}(9.1 .2)$, and one sees ow $f_{12}$ ow $f_{11}$, ow $f_{21}-$ ow $f_{22} \in \operatorname{ow}\left(C^{\sharp}\right)$ by (9.1.2) and (9.1.3). Since $f_{i j}$ have representatives of the form

$$
\text { (invariant unit) } \cdot(\text { monomial in } x \text { ) }
$$

in $\mathscr{O}_{X^{\sharp}, P^{\sharp}}$ (cf. (2.7)), this implies that $f_{12} / f_{11}, f_{21} / f_{22} \in \mathscr{O}_{C^{\sharp}, P^{\sharp}}$ and $\left(f_{21} / f_{22}\right)\left(P^{\sharp}\right)=0$ by $(2.7)$ and $u_{1}=v_{1}+\left(f_{12} / f_{11}\right) v_{2}$ and $u_{2}=\left(f_{21} / f_{22}\right) v_{1}+v_{2}$ form an $\ell$-free $\ell$-basis of $\mathscr{E}$ at $P$ and the claim follows.

(9.1.6) Since ow $f_{21}-$ ow $f_{11} \in \mathrm{ow}\left(C^{\sharp}\right)$, there exists $\mu \in \mathbb{C}$ such that

$$
\operatorname{ord}\left(\mu f_{11}+f_{21}\right)>\text { ord } f_{11}
$$

If $s_{3} \in \mathscr{E} \otimes \mathscr{O}_{C, P}$ satisfies $s_{3}(P)=\mu s_{1}(P)+s_{2}(P)$, then $\mathscr{E}=\mathscr{O}_{C} s_{1} \oplus \mathscr{O}_{C} s_{3}$ near $P$ and the coefficients $f_{31}$ and $f_{32}$ in $s_{3}=f_{31} v_{1}+f_{32} v_{2}$ satisfy ord $f_{31}>$ ord $f_{11}$. Hence we can apply (9.1.5) to $s_{1}, s_{3}$.

(9.1.7) Corollary. Assume that $\left(C^{\sharp}, P^{\sharp}\right)$ and $\left(C^{b}, P^{b}\right)$ are smooth (resp. $P$ and $P^{\prime}$ are ordinary). Let $\mathscr{E}$ be a torsion-free $\ell$-coherent $\mathscr{O}_{C}$-module of rank 2 (resp. $\mathscr{E}=\operatorname{gr}_{C}^{0} I$ ). Then

(i) $\mathscr{E}$ is a locally $\ell$-free $\mathscr{O}_{C}$-module of rank 2 ,

(ii) every $\mathscr{E}$-saturated invertible $\mathscr{O}_{C}$-submodule $\mathscr{L}$ of $\mathscr{E}(2.2)$ is an $\ell$-invertible $\mathscr{O}_{C}$-module with its induced $\ell$-structure, and

(iii) if $\mathscr{E}=\mathscr{L} \oplus \mathscr{M}$ is a splitting into invertible $\mathscr{O}_{C}$-modules such that $\operatorname{deg} \mathscr{L}>\operatorname{deg} \mathscr{M}$, then we have an l-splitting $\mathscr{E}=\mathscr{L} \widetilde{\oplus}\left\{\left(\operatorname{id}_{M}, f\right)(\mathscr{M})\right\}$ for some $f \in \operatorname{Hom}(\mathscr{L}, \mathscr{M})$, where $\left(\operatorname{id}_{M}, f\right): \mathscr{M} \rightarrow \mathscr{L} \oplus \mathscr{M}=\mathscr{E}$.

Proof. (i) and (ii) follow from (9.1.1(i)) and (9.1.1(ii)), respectively. For (ii), we see that $\mathscr{M}^{*} \otimes \mathscr{L}$ is generated by global sections and $\operatorname{dim} H^{0}\left(\mathscr{M}^{*} \otimes \mathscr{L}\right) \geq 2$ by $\operatorname{deg} \mathscr{L}>\operatorname{deg} \mathscr{M}$, whence (iii) follows from (9.1.1(ii)). 
(9.1.8) Corollary. Assume that $\left(C^{\sharp}, P^{\sharp}\right)$ and $\left(C^{b}, P^{b}\right)$ are smooth. Let $\mathscr{E}, \mathscr{L}$, $\mathscr{M}$ be $\ell$-free $\mathscr{O}_{C}$-modules of rank $2,1,1$, and let

$$
0 \rightarrow \mathscr{L} \rightarrow \mathscr{E} \rightarrow \mathscr{M} \rightarrow 0
$$

be an $\ell$-exact sequence. Then

(i) for a point $Q$ such that $q l \operatorname{deg}(\mathscr{L}, Q) \geq q l \operatorname{deg}(\mathscr{M}, Q)$, an arbitrary splitting $\mathscr{E}=\mathscr{L} \oplus \mathscr{N}$ at $Q$ is an $\ell$-splitting at $Q$, and

(ii) if (a) $\operatorname{deg} \mathscr{L}>\operatorname{deg} \mathscr{M}$, or

(b) $\operatorname{deg} \mathscr{L} \geq \operatorname{deg} \mathscr{M}-1$ and $q l \operatorname{deg}(\mathscr{L}, Q) \geq q l \operatorname{deg}(\mathscr{M}, Q)$ for $Q=P$ and $P^{\prime}$,

then the sequence is $\ell$-split.

Proof. As for (ii), (a) follows from (9.1.7), and (b) follows from (i). As for (i), it is enough to prove that a splitting $\mathscr{E}=\mathscr{L} \oplus \mathscr{N}$ at $P$ is an $\ell$-splitting at $P$ assuming that $q l \operatorname{deg}(\mathscr{L}, P) \geq q l \operatorname{deg}(\mathscr{M}, P)$. Let $\left\{u_{1}, u_{2}\right\}$ be an $\ell$-free $\ell$-basis for $\mathscr{E}$ at $P$ such that $u_{1}$ is an $\ell$-free $\ell$-basis for $\mathscr{L}$ at $P$. Then $R\left(\right.$ wt $\left.u_{1}\right) \geq R\left(\right.$ wt $\left.u_{2}\right)(8.8 .5)$ by the assumption. We note that $\mathscr{N}$ is generated by $f_{1} u_{1}+f_{2} u_{2}$ such that $f_{1}, f_{2} \in \mathscr{O}_{C^{\sharp}, P^{\sharp}}$ are semi-invariants with ord $f_{1} \geq$ $R\left(\right.$ wt $\left.u_{1}\right)$ and ord $f_{2}=R\left(\right.$ wt $\left.u_{2}\right)$. Thus $f_{1} / f_{2} \in \mathscr{O}_{C^{\sharp}, P^{\sharp}}$ and $\left\{u_{1},\left(f_{1} / f_{2}\right) u_{1}+u_{2}\right\}$ is an $\ell$-free $\ell$-basis for $\mathscr{E}$ at $P$, and $\mathscr{E}=\mathscr{L} \widetilde{\oplus} \mathscr{N}$.

(9.2) Under the notation and assumptions of (9.1), assume that $P$ and $P^{\prime}$ are ordinary. Then they are of type (IA) or $\left(\mathrm{IA}^{\vee}\right)$ and $i_{P}(1)=\operatorname{siz}_{P}=1$, $i_{P^{\prime}}(1)=\operatorname{siz}_{P^{\prime}}=1$ by $(*)$ in (9.1) and (4.10). Hence $\operatorname{deg} \operatorname{gr}_{C}^{1} \mathscr{O}=-1$ (2.3.2) and $\operatorname{gr}_{C}^{1} \mathscr{O} \simeq \mathscr{O} \oplus \mathscr{O}(-1)$, since $H^{1}\left(\operatorname{gr}_{C}^{1} \mathscr{O}\right)=0$ (2.3.4). We note that the subsheaf $\mathscr{L} \subset \operatorname{gr}_{C}^{1} \mathscr{O}$ such that $\mathscr{L} \simeq \mathscr{O}$ is unique and that $\mathscr{L}$ is an $\ell$ invertible $\mathscr{O}_{C}$-module, and let $u_{1}\left(\right.$ resp. $\left.u_{1}^{\prime}\right)$ be an $\ell$-free $\ell$-basis of $\mathscr{L}$ at $P$ (resp. $\left.P^{\prime}\right)(8.8 .3)$.

(9.3) Theorem. Assume that $P$ and $P^{\prime}$ are ordinary as in $(9.2)$. Then $\left(C^{\sharp}, P^{\sharp}\right)$ and $\left(C^{b}, P^{b}\right)$ are smooth (whence $P$ and $P^{\prime}$ are primitive), wt $u_{1} \equiv-1(m)$, and $\mathrm{wt}^{\prime} u_{1}^{\prime} \equiv-1\left(m^{\prime}\right)$. Furthermore, $a_{1}=a_{1}^{\prime}=1$ after necessary permutations are made as in (4.2) .

(9.3.1) Remark. By (4.5.1), an $\ell$-free $\ell$-basis of $\operatorname{gr}_{C}^{1} \mathscr{O}$ at $P$ has wt $\equiv s a_{1} a_{2}$, $-a_{1}(\bmod m)$. If $P$ is of type $\left(\mathrm{IA}^{\vee}\right)$, then we treat it as a separate case:

$$
s \geq 2 \text {, }
$$

and then consider locally primitive $X \supset C$ as follows.

We note that if $a_{2}=1$, then one may permute $x_{1}$ and $x_{3}$ (9.1), by which $a_{1} a_{2} \equiv a_{1}$ and $a_{3}$ are permuted. We also note that if $a_{2}=m-1$, then $a_{1} a_{2} \equiv a_{3}$. Thus one may assume either (i) wt $u_{1} \equiv a_{1} a_{2}$ and $2 \leq a_{2} \leq m-2$, or (ii) wt $u_{1} \equiv a_{3}$, and the same remark applies to $P^{\prime}$. Therefore there are three cases (modulo permutation of $P$ and $P^{\prime}$ ):

(9.3.a) wt $u_{1} \equiv a_{1} a_{2}, \quad a_{2} \not \equiv \pm 1(m) ; \quad \mathrm{wt}^{\prime} u_{1}^{\prime} \equiv a_{1}^{\prime} a_{2}^{\prime}, \quad a_{2}^{\prime} \not \equiv \pm 1\left(m^{\prime}\right)$, 


$$
\text { wt } u_{1} \equiv a_{1} a_{2}, \quad a_{2} \not \equiv \pm 1(m) ; \quad \text { wt }^{\prime} u_{1}^{\prime} \equiv a_{3}^{\prime}\left(m^{\prime}\right) \text {, }
$$

and

$$
\text { wt } u_{1} \equiv a_{3}(m) ; \quad \text { wt }^{\prime} u_{1}^{\prime} \equiv a_{3}^{\prime}\left(m^{\prime}\right) \text {. }
$$

Proof. We will prove (9.3) by showing that only (9.3.c) is possible and that $a_{1}=a_{1}^{\prime}=1$. Since $\operatorname{gr}_{C}^{1} \mathscr{O}=\mathscr{L} \oplus \mathscr{O}(-1)$ and $\mathscr{L} \simeq \mathscr{O}(9.2)$, one may choose subbundle $\mathscr{M} \simeq \mathscr{O}(-1)$ of $\operatorname{gr}_{C}^{1} \mathscr{O}$ such that $\operatorname{gr}_{C}^{1} \mathscr{O}=\mathscr{L} \oplus \mathscr{M}$ is an $\ell$-splitting (9.1.7(iii)). Let $u_{2}$ (resp. $u_{2}^{\prime}$ ) be an $\ell$-free $\ell$-basis of $\mathscr{M}$ at $P$ (resp. $P^{\prime}$ ).

(9.3.2) Let $J$ be the $C$-laminal ideal of width 2 such that $J / I^{(2)}=\mathscr{L}$. Since $J / I^{(2)}=\mathscr{L}$, the surjection $\alpha_{J}: \operatorname{gr}_{C}^{0} J \rightarrow J / I^{(2)}=\mathscr{L}$ induced by identity is an $\ell$-surjection by (8.8.1). Thus we have equalities $\operatorname{Im} \alpha_{J}=\operatorname{Ker} \beta_{J}=\mathscr{L}$ with $\ell$-structure by $(8.4(\mathrm{ii}))$ and an $\ell$-isomorphism $\operatorname{gr}^{1}(\mathscr{O}, J) \simeq \mathscr{M}$. In particular $J$ is a nested c.i. outside $\left\{P, P^{\prime}\right\}$ by $(8.4(\mathrm{i}))$.

Let $J^{\sharp}$ (resp. $\left.J^{b}\right)$ be the canonical lifting of $J$ at $P$ (resp. $P^{\prime}$ ). We may replace $u_{1}, u_{2} \in I^{\sharp} / I^{\sharp(2)}$ (resp. $u_{1}^{\prime}, u_{2}^{\prime} \in I^{\mathrm{b}} / I^{\mathrm{b}(2)}$ ) with their representatives so that we may assume $u_{1} \in J^{\sharp}$ and $u_{2} \in I^{\sharp}$ (resp. $u_{1}^{\prime} \in J^{\mathrm{b}}$ and $u_{2}^{\prime} \in I^{\mathrm{b}}$ ). Thus one sees $I^{\sharp}=\left(u_{1}, u_{2}\right)$ and $J^{\sharp}=\left(u_{1}, u_{2}^{2}\right)$ (resp. $I^{b}=\left(u_{1}^{\prime}, u_{2}^{\prime}\right)$ and $\left.J^{b}=\left(u_{1}^{\prime}, u_{2}^{\prime 2}\right)\right)$ by $(8.3)$, and $\left(u_{2}, u_{1}\right)$ (resp. $\left.\left(u_{2}^{\prime}, u_{1}^{\prime}\right)\right)$ is a $(1,2)$-monomializing $\ell$-basis for $I \supset J$ at $P$ (resp. $\left.P^{\prime}\right)$. Hence

$$
\begin{aligned}
0 \leq & l \operatorname{deg}_{C} \mathscr{M}+\frac{1}{2} l \operatorname{deg}_{C} \mathscr{L} \\
= & \left(-1+\frac{1}{\bar{m}} R\left(\mathrm{wt} u_{2}\right)+\frac{1}{m^{\prime}} R^{\prime}\left(\mathrm{wt}^{\prime} u_{2}^{\prime}\right)\right) \\
& +\frac{1}{2}\left(\frac{1}{\bar{m}} R\left(\mathrm{wt} u_{1}\right)+\frac{1}{m^{\prime}} R^{\prime}\left(\mathrm{wt}^{\prime} u_{1}^{\prime}\right)\right)
\end{aligned}
$$

by (8.12). By (2.3) and (4.9(i)), one has

$$
1<a_{2} \cdot \bar{m}^{-1}+a_{2}^{\prime} \cdot m^{\prime-1} \text {. }
$$

(9.3.5) Case (9.3.ipr). We will disprove this case. Since $\operatorname{siz}_{P}=i_{P}(1)=1$, we have $w_{P}(0) \geq 2 / 3$ by $(5.6(\mathrm{ii}))$. Whence $w_{P^{\prime}}(0)<1 / 3$ by $(2.3 .3)$ and $a_{1}^{\prime}=1$ by $(5.1(\mathrm{i}))$. Since $\operatorname{siz}_{P}=\operatorname{siz}_{P^{\prime}}=1$, we see that $\left\{R\left(\right.\right.$ wt $\left.u_{1}\right), R\left(\right.$ wt $\left.\left.u_{2}\right)\right\}$ $=\left\{\bar{m}-s a_{1} a_{2}, a_{1}\right\}$ and $a_{1} \leq \bar{m}-s a_{1} a_{2}$ by (4.5.2) and that $\left\{R^{\prime}\left(\mathrm{wt}^{\prime} u_{1}^{\prime}\right)\right.$, $\left.R^{\prime}\left(\mathrm{wt}^{\prime} u_{2}^{\prime}\right)\right\}=\left\{m^{\prime}-a_{2}^{\prime}, 1\right\}$ and $1 \leq m^{\prime}-a_{2}^{\prime}$. Then (9.3.3) gives

$$
\begin{aligned}
0 & \leq 2\left(-1+\frac{\bar{m}-s a_{1} a_{2}}{\bar{m}}+\frac{m^{\prime}-a_{2}^{\prime}}{m^{\prime}}\right)+\frac{a_{1}}{\bar{m}}+\frac{1}{m^{\prime}} \\
& <\bar{m}^{-1}\left(-2 s a_{1} a_{2}+a_{1}+3 a_{2}\right),
\end{aligned}
$$

where $1 / m^{\prime} \leq\left(m^{\prime}-a_{2}^{\prime}\right) / m^{\prime}<a_{2} \cdot \bar{m}^{-1} \quad(9.3 .4)$ is used in the second inequality. Hence we have a contradiction:

$$
\begin{aligned}
0 & <-2 s a_{1} a_{2}+a_{1}+3 a_{2} \leq-4 a_{1} a_{2}+a_{1}+3 a_{2} \\
& =a_{1}\left(1-4 a_{2}\right)+3 a_{2} \leq 1 \cdot\left(1-4 a_{2}\right)+3 a_{2}=1-a_{2} \leq 0
\end{aligned}
$$


by $s \geq 2, a_{1}, a_{2} \geq 1$. Thus case (9.3.ipr) does not occur, and $P$ is a primitive point and $\bar{m}=m$ in the rest of proof of (9.3).

(9.3.6) Case (9.3.a). We will disprove this case. By $U\left(a_{1} a_{2}\right)=1$ and $U^{\prime}\left(a_{1}^{\prime} a_{2}^{\prime}\right)$ $=1$, one has $R\left(\mathrm{wt} u_{1}\right)=m-a_{1} a_{2}$ and $R^{\prime}\left(\mathrm{wt}^{\prime} u_{1}^{\prime}\right)=m^{\prime}-a_{1}^{\prime} a_{2}^{\prime}$, and by definition one has $R\left(\mathrm{wt} u_{2}\right)=a_{1}$ and $R^{\prime}\left(\mathrm{wt}^{\prime} u_{2}^{\prime}\right)=a_{1}^{\prime}$. Thus (9.3.3) reduces to

$$
0 \leq \frac{1}{2 m} a_{1}\left(2-a_{2}\right)+\frac{1}{2 m^{\prime}} a_{1}^{\prime}\left(2-a_{2}^{\prime}\right)
$$

which implies $a_{2}=a_{2}^{\prime}=2$, because $a_{2}, a_{2}^{\prime} \geq 2$. By $a_{2} \not \equiv \pm 1(m)$ and $\left(a_{2}, m\right)=1$, one sees that $m \geq 5$, and similarly $m^{\prime} \geq 5$. This contradicts (9.3.4).

(9.3.7) Case (9.3.b). We will disprove this case. We have $R\left(\right.$ wt $\left.u_{1}\right)=m-a_{1} a_{2}$ and $R\left(\right.$ wt $\left.u_{2}\right)=a_{1}, R^{\prime}\left(\mathrm{wt}^{\prime} u_{1}^{\prime}\right)=a_{1}^{\prime}$ and $R^{\prime}\left(\mathrm{wt}^{\prime} u_{2}^{\prime}\right)=m^{\prime}-a_{1}^{\prime} a_{2}^{\prime}$, and $m \geq 5$ as above. Thus (9.3.3) reduces to

$$
1 \geq a_{1}\left(a_{2}-2\right) / m+a_{1}^{\prime}\left(2 a_{2}^{\prime}-1\right) / m^{\prime} \text {. }
$$

(9.3.7.2) Claim $a_{1}^{\prime}=1$. First by $a_{2} \not \equiv \pm 1$ and $a_{2}<m$, one has $m \geq a_{2}+2$ and $1=m / m \geq 2 / m+a_{2} / m>2 / m+1-a_{2}^{\prime} / m^{\prime}$ by (9.3.4), whence $0>2 / m-a_{2}^{\prime} / m^{\prime}$. By (9.3.7.1), one has

$$
1 \geq\left(a_{2}-2\right) / m+a_{1}^{\prime}\left(2 a_{1}^{\prime}-1\right) / m^{\prime}>1-a_{2}^{\prime} / m^{\prime}-2 / m+a_{1}^{\prime}\left(2 a_{2}^{\prime}-1\right) / m^{\prime},
$$

where we used (9.3.4) in the second inequality, whence

$$
2 / m-a_{2}^{\prime} / m^{\prime}>-2 a_{2}^{\prime} / m^{\prime}+a_{1}^{\prime}\left(2 a_{2}^{\prime}-1\right) / m^{\prime}
$$

Hence one has

$$
0>2 / m-a_{2}^{\prime} / m^{\prime}>-2 a_{2}^{\prime} / m^{\prime}+a_{1}^{\prime}\left(2 a_{2}^{\prime}-1\right) / m^{\prime}=\left\{\left(a_{1}^{\prime}-1\right)\left(2 a_{2}^{\prime}-1\right)-1\right\} / m^{\prime} \text {. }
$$

Whence $0>\left(a_{1}^{\prime}-1\right)\left(2 a_{2}^{\prime}-1\right)-1 \geq\left(a_{1}^{\prime}-1\right)-1=a_{1}^{\prime}-2$. Thus $a_{1}^{\prime}=1$ and (9.3.7.2) is proved.

(9.3.7.3) Claim $a_{1}=1$. We first consider the case $m=5$. If $a_{1} \geq 2$, then $a_{1}=a_{2}=2$ by $R\left(\right.$ wt $\left.u_{1}\right)=5-a_{1} a_{2}>0$, which contradicts $5 \in a_{1} \mathbb{Z}_{+}+a_{2} \mathbb{Z}_{+}$. Thus $a_{1}=1$ if $m=5$. Let us assume $m \geq 6$ and $a_{1} \geq 2$. Then by (9.3.7.1), one has

$$
1 \geq 2\left(a_{2}-2\right) / m+\left(2 a_{2}^{\prime}-1\right) / m^{\prime}>2-4 / m-1 / m^{\prime}
$$

where (9.3.4) is used in the second inequality. Thus by $m \geq 6$, one has $1 / m^{\prime}>$ $1-4 / m \geq 1 / 3$, which contradicts our assumption $m^{\prime} \geq 3$. Hence $a_{1}=1$ if $m \geq 6$, and (9.3.7.3) is proved.

(9.3.7.4) Thus $\left(C^{\sharp}, P^{\sharp}\right)$ and $\left(C^{b}, P^{b}\right)$ are smooth, whence $U(z)=\lceil z / m\rceil$ and $U^{\prime}(z)=\left\lceil z^{\prime} / m^{\prime}\right\rceil \quad(z \in \mathbb{Z})$ and $T L\left(z+z^{\sharp} P^{\sharp}+z^{b} P^{b}\right)=z+\left[z^{\sharp} / m\right]+\left[z^{b} / m^{\prime}\right]$ (cf. (8.9.1(iii)) for definition). One also sees $q l_{C}(\mathscr{L})=\left(m-a_{2}\right) P^{\sharp}+P^{b}$ and $q l_{C}(\mathscr{M})=-1+P^{\sharp}+\left(m^{\prime}-a_{2}^{\prime}\right) P^{b}$ by (8.8.5) (cf. (8.9.1(ii)) for definition). 
(9.3.7.5) Claim $2 a_{2}^{\prime}<m^{\prime}$. We will derive a contradiction assuming that $2 a_{2}^{\prime} \geq$ $m^{\prime}$. We note that if $2 a_{2}^{\prime}=m^{\prime}$ then $m^{\prime}=2$ and $a_{2}^{\prime}=1$ by $\left(a_{2}^{\prime}, m^{\prime}\right)=1$. This contradicts $m^{\prime} \geq 3$, and we have $2 a_{2}^{\prime}-1 \geq m^{\prime}$. Hence by (9.3.7.1), we have $a_{2}=2$ and $2 a_{2}^{\prime}=m^{\prime}+1$, whence $m^{\prime} \equiv 1$ (2). Then (9.3.4) reduces to $1<2 / m+\left(m^{\prime}+1\right) / 2 m^{\prime}$, i.e. $1<4 / m+1 / m^{\prime}$. Since $m \geq 5$, one has $m^{\prime}<5$ by $1 / m^{\prime}>1-4 / m \geq 1 / 5$. Thus by $m^{\prime} \equiv 1$ (2) and $m^{\prime}>1$, one has $m^{\prime}=3$, whence $m=5$ by $4 / m>1-1 / 3=2 / 3$. Since $a_{2}=2$ and $a_{2}^{\prime}=\left(m^{\prime}+1\right) / 2=2$, one sees $q l_{C}(\mathscr{L})=3 P^{\sharp}+P^{b}$ and $q l_{C}(\mathscr{M})=-1+P^{\sharp}+P^{b}$ (9.3.7.4). Hence subquotients of $F^{1}(\mathscr{O}, J) / F^{4}(\mathscr{O}, J)$ fit in exact sequences $(8.6)$

$$
\begin{gathered}
\operatorname{gr}^{1}(\mathscr{O}, J)=\mathrm{gr}^{1,0}(\mathscr{O}, J), \\
0 \rightarrow \mathrm{gr}^{2,1}(\mathscr{O}, J) \rightarrow \operatorname{gr}^{2}(\mathscr{O}, J) \rightarrow \mathrm{gr}^{2,0}(\mathscr{O}, J) \rightarrow 0, \\
0 \rightarrow \mathrm{gr}^{3,1}(\mathscr{O}, J) \rightarrow \operatorname{gr}^{3}(\mathscr{O}, J) \rightarrow \mathrm{gr}^{3,0}(\mathscr{O}, J) \rightarrow 0 .
\end{gathered}
$$

Since $J$ is a nested c.i. outside $\left\{P, P^{\prime}\right\}$ and $(1,2)$-monomializable at $P$ and $P^{\prime}$ (9.3.2), we can apply (8.12(ii)) (with $d=2, s=s^{\prime}=0$ ) to get

$$
\begin{array}{ll}
\operatorname{gr}^{1,0}(\mathscr{O}, J) \simeq \mathscr{M} & (n=1, q=0, r=1), \\
\operatorname{gr}^{2,0}(\mathscr{O}, J) \simeq \mathscr{L} & (n=2, q=1, r=0), \\
\operatorname{gr}^{2,1}(\mathscr{O}, J) \simeq \mathscr{M}^{\widetilde{\otimes} 2}, & \\
\operatorname{gr}^{3,0}(\mathscr{O}, J) \simeq \mathscr{L} \widetilde{\otimes} \cdot \mathscr{M} & (n=3, q=1, r=1), \\
\operatorname{gr}^{3,1}(\mathscr{O}, J) \simeq \mathscr{M}^{\widetilde{\otimes} 3} . &
\end{array}
$$

These are calculated and arranged as

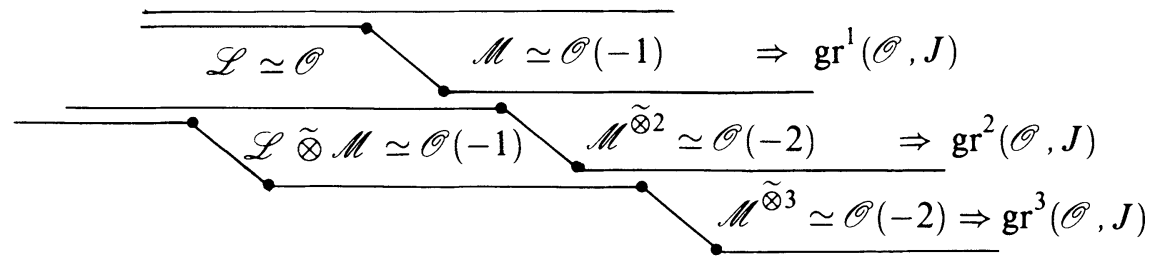

because $q l_{C}(\mathscr{L} \tilde{\otimes} \mathscr{M})=q l_{C}(\mathscr{L})+q l_{C}(\mathscr{M})=-1+4 P^{\sharp}+2 P^{b}, q l_{C}\left(\mathscr{M}^{\widetilde{\otimes} 2}\right)=$ $2 q l_{C}(\mathscr{M})$

$=-2+2 P^{\sharp}+2 P^{b}$, and $q l_{C}\left(\mathscr{M}^{\widetilde{\otimes} 3}\right)=3 q l_{C}(\mathscr{M})=-2+3 P^{\sharp}$ are normalized expressions $(8.9 .1)$. Hence $\chi\left(F^{1}(\mathscr{O}, J) / F^{4}(\mathscr{O}, J)\right)=-1<0$, which is a contradiction to $H^{1}(\mathscr{O})=0$ as in (8.7). Thus (9.3.7.5) is proved.

(9.3.7.6) By (9.3.7.5), one has $q l_{C}\left(\mathscr{M}^{\widetilde{\otimes} 2}\right)=2 q l_{C}(\mathscr{M})=-1+2 P^{\sharp}+\left(m^{\prime}-2 a_{2}^{\prime}\right) P^{b}$ and $\operatorname{deg}_{C} \mathscr{M}^{\widetilde{\otimes} 2}=-1$. Thus the $\mathscr{O}_{C}$-module exact sequence

$$
0 \rightarrow \mathscr{M}^{\widetilde{\otimes} 2} \rightarrow \mathrm{gr}_{C}^{0} J \rightarrow \mathscr{L} \rightarrow 0
$$


is split, and $\operatorname{gr}_{C}^{0} J$ contains a unique subbundle $\mathscr{L}_{J}$ such that $\mathscr{L}_{J} \stackrel{\sim}{\longrightarrow} \mathscr{L}$ and $\mathrm{gr}_{C}^{0} J=\mathscr{M}^{\tilde{\otimes} 2} \oplus \mathscr{L}_{J}$ as $\mathscr{O}_{C}$-modules.

Let $K$ be the $C$-primary ideal such that $J \supset K \supset F^{1} J$ and $K / F^{1} J=$ $\mathscr{L}_{J}$. Then $K$ is a $C$-laminal ideal of width 3 which is a nested c.i. outside $\left\{P, P^{\prime}\right\}$, and we have $\ell$-isomorphisms $\operatorname{Ker} \beta_{K}=\operatorname{Ker} \beta_{J}=\mathscr{L}$ and $\operatorname{gr}^{1}(\mathscr{O}, K)=$ $\operatorname{gr}^{1}(\mathscr{O}, J) \simeq \mathscr{M}$ by (8.14). We see that $\operatorname{gr}_{C}^{0} J=\mathscr{L}_{J} \oplus \mathscr{M}^{\widetilde{\otimes} 2}$ is an $\ell$-splitting at $P^{\prime}$ by $(9.1 .8(\mathrm{i}))$, because $q l \operatorname{deg}\left(\mathscr{M}^{\widetilde{\otimes} 2}, P^{b}\right)=m^{\prime}-2 a_{2}^{\prime} \geq q l \operatorname{deg}\left(\mathscr{L}, P^{b}\right)=1$ by (9.3.7.4) and $2 a_{2}^{\prime}<m^{\prime}$ (9.3.7.5). Hence the $(1,2)$-monomializing $\ell$-basis $\left(u_{2}^{\prime}, u_{1}^{\prime}\right)$ of $I \supset J$ at $P^{\prime}$ lifts to a $(1,3)$-monomializing $\ell$-basis (denoted by the same $\left(u_{2}^{\prime}, u_{1}^{\prime}\right)$, by abuse of language) of $I \supset K$ at $P^{\prime}$ (8.15.1). Let

$$
\left(\mathscr{L}_{J}\right)_{P}=\mathscr{L}_{J} \otimes \mathscr{O}_{C, P}=\left(\mu t^{m-a_{2}} u_{1}+t^{m k+2} u_{2}^{2}\right) \text {, }
$$

where $\mu$ is a unit and $0 \leq k \leq \infty$. If $k=0$ (resp. $k \geq 1$ ), then $u_{2}$, $v=\mu x_{1}^{m-a_{2}-2} u_{1}+u_{2}{ }^{2}, u_{1}$ form a $(1,3,2)$-monomializing $\ell$-basis of the first kind (resp. $\left(u_{2}, u_{1}\right)$ lifts to a $(1,3)$-monomializing $\ell$-basis denoted by the same $\left(u_{2}, u_{1}\right)$, by abuse of language) of $I \supset K$ at $P$ by $m-a_{2} \geq 2$ and (8.15), where $\left.x_{1}\right|_{C^{\sharp}}=t$ by $a_{1}=1$ (9.1). One can see $\ell$-isomorphisms

$$
\mathscr{L}_{J}=\mathrm{gr}^{3,0}(\mathscr{O}, K) \simeq \mathscr{L} \widetilde{\otimes} \mathscr{D}^{\widetilde{\otimes}(-\varepsilon)}
$$

by (8.14.1) (with $d=2$ ) for the first equality and by (8.12(ii)) (with $d=n=3$, $q=1, r=i=0$ ) for the second, where $\varepsilon=1$ (resp. 0 ) and $\mathscr{D}$ is as defined in $(8.11 .1)$ :

$$
\mathscr{D}_{P}=\mathscr{O}_{C, P} \subset \mathscr{D}_{P}^{\sharp}=\mathscr{O}_{C^{\sharp}, P^{\sharp}} t^{-\left(m-a_{2}-2\right)}
$$

(note $m>m-a_{2}-2 \geq 0$ ).

(9.3.7.7) Claim $a_{2}^{\prime}=1$. Indeed by (9.3.7.6) and (8.12), one has

$$
0 \leq l \operatorname{deg}_{C} \mathscr{M}+\frac{1}{3} l \operatorname{deg}_{C} \mathscr{L}+\varepsilon\left(\frac{1}{2}-\frac{1}{3}\right) \frac{1}{m}\left(m-a_{2}-2\right)
$$

under the notation of (9.3.7.6). Thus in any case, we have

$$
0 \leq\left(1 / m-a_{2}^{\prime} / m^{\prime}\right)+\left(\left(m-a_{2}\right) / m+1 / m^{\prime}\right) / 3+\left(m-a_{2}-2\right) / 6 m,
$$

whence $m^{\prime}\left(3\left(m-a_{2}\right)+4\right) \geq 2 m\left(3 a_{2}^{\prime}-1\right)$. Thus one sees

$$
\frac{m-a_{2}}{a_{2}^{\prime}} m^{\prime}<m \leq \frac{3\left(m-a_{2}\right)+4}{2 \cdot\left(3 a_{2}^{\prime}-1\right)} m^{\prime},
$$

where (9.3.4) was used for the first inequality. Whence follows $\left\{3\left(m-a_{2}\right)+\right.$ $4\} \cdot a_{2}^{\prime}>2\left(3 a_{2}^{\prime}-1\right)\left(m-a_{2}\right)$, hence $4 a_{2}^{\prime}>\left(m-a_{2}\right)\left(3 a_{2}^{\prime}-2\right) \geq 2\left(3 a_{2}^{\prime}-2\right)$ by $m-a_{2} \geq 2$, whence $a_{2}^{\prime}=1$. Thus (9.3.7.7) is proved.

(9.3.7.8) By (8.12(ii)) (with $d=n=3, q=i=1, r=0$ ), we see $\mathrm{gr}^{3.1}(\mathscr{O}, K)$ $=\mathscr{M}^{\tilde{\otimes} 3} \widetilde{\otimes} \mathscr{D}{ }^{\widetilde{\otimes} \varepsilon}$. Hence by $\mathscr{L}_{J}=\mathrm{gr}^{3,0}(\mathscr{O}, K)(9.3 .7 .6)$, we have an $\ell$-exact sequence

$$
0 \rightarrow \mathscr{M}^{\widetilde{\otimes} 3} \widetilde{\otimes} \mathscr{D}^{\tilde{\otimes} \varepsilon} \rightarrow \mathrm{gr}_{C}^{0} K \rightarrow \mathscr{L}_{J} \rightarrow 0,
$$


where $\operatorname{deg}_{C} \mathscr{M}^{\widetilde{\otimes} 3} \widetilde{\otimes} \mathscr{D}^{\widetilde{\otimes} \varepsilon}=-1$ and $\operatorname{deg}_{C} \mathscr{L}_{J}=0$ by

$$
\begin{aligned}
q l_{C}\left(\mathscr{M}^{\tilde{\otimes} 3} \tilde{\otimes} \mathscr{D}^{\tilde{\otimes} \varepsilon}\right) & =3\left\{-1+P^{\sharp}+\left(m^{\prime}-1\right) P^{b}\right\}+\varepsilon\left(m-a_{2}-2\right) P^{\sharp} \\
& =\left\{-1+3 P^{\sharp}+\left(m^{\prime}-3\right) P^{b}\right\}+\varepsilon\left(m-a_{2}-2\right) P^{\sharp} \\
& =-1+\left\{3+\varepsilon\left(m-a_{2}-2\right)\right\} P^{\sharp}+\left(m^{\prime}-3\right) P^{b}, \\
q l_{C}\left(\mathscr{L}_{J}\right) & =q l_{C} \mathscr{L}-\varepsilon\left(m-a_{2}-2\right) P^{\sharp} \\
& =\left\{\left(m-a_{2}\right)-\varepsilon\left(m-a_{2}-2\right)\right\} P^{\sharp}+P^{b},
\end{aligned}
$$

where $0<3+\varepsilon\left(m-a_{2}-2\right)<m$ and $0<\left(m-a_{2}\right)-\varepsilon\left(m-a_{2}-2\right)<m$ by $2 \leq a_{2} \leq m-2$. Hence the exact sequence splits, and we write $\operatorname{gr}_{C}^{0} K=$ $\mathscr{M}^{\widetilde{\otimes} 3} \widetilde{\otimes} \mathscr{D}^{\widetilde{\otimes} \varepsilon} \oplus \mathscr{L}_{K}$ as $\mathscr{O}_{C}$-modules.

Let $H$ be a $C$-primary ideal such that $K \supset H \supset F^{1} K$ and $H / F^{1} K=\mathscr{L}_{K}$. Then $H$ is a $C$-laminal ideal of width 4 which is a nested c.i. outside $\left\{P, P^{\prime}\right\}$, and we have $\ell$-isomorphisms $\operatorname{Ker} \beta_{H}=\operatorname{Ker} \beta_{K}=\mathscr{L}$ and $\operatorname{gr}^{1}(\mathscr{O}, H)=$ $\operatorname{gr}^{1}(\mathscr{O}, K) \simeq \mathscr{M}$ by $(8.14)$.

(9.3.7.9) Claim $\operatorname{gr}_{C}^{0} K=\mathscr{M}^{\widetilde{\otimes} 3} \widetilde{\otimes} \mathscr{D}^{\widetilde{\otimes} \varepsilon} \oplus \mathscr{L}_{K}$ is an $\ell$-splitting at $P$ if $\varepsilon=1$. Indeed since $q l \operatorname{deg}\left(\mathscr{M}^{\widetilde{\otimes} 3} \tilde{\otimes} \mathscr{D}, P\right)=m+1-a_{2}>2=q l \operatorname{deg}\left(\mathscr{L}_{J}, P\right)$, it is an $\ell$-splitting at $P$ by $(9.1 .8(\mathrm{i}))$.

(9.3.7.10) The $(1,3,2)$-monomializing $\ell$-basis $\left(u_{2}, v, u_{1}\right)$ of the first kind (resp. $(1,3)$-monomializing $\ell$-basis $\left.\left(u_{2}, u_{1}\right)\right)$ induces a $(1,4,2)$-monomializing $\ell$ basis $\left(u_{2}, v, u_{1}\right)$ of the first kind with the same attached semi-invariant (resp. $(1,4,3)$-monomializing $\ell$-basis of the first kind or $(1,4)$-monomializing $\ell$ basis as in (9.3.7.6)) for $I \supset H$ at $P$ by (8.16) (resp. by (8.15)). At $P^{\prime}$, one has

$$
\left(\mathscr{L}_{K}\right)_{P^{\prime}}=\mathscr{L}_{P^{\prime}}=\mathscr{O}_{C^{\prime}, P^{\prime}}\left(\mu^{\prime} t^{\prime} u_{1}^{\prime}+t^{m^{\prime} k^{\prime}+m^{\prime}-3} u_{2}^{\prime 3}\right),
$$

where $\mu^{\prime}$ is a unit and $0 \leq k^{\prime} \leq \infty$. If $k^{\prime} \geq 1$ or $m^{\prime} \geq 4$, then $\operatorname{gr}_{C}^{0} K=$ $\mathscr{L}_{K} \oplus \mathscr{M}^{\widetilde{\otimes} 3} \tilde{\otimes} \mathscr{D}^{\widetilde{\otimes} \varepsilon}$ is an $\ell$-splitting at $P^{\prime}$ and $H$ is $(1,4)$-monomializable at $P^{\prime}(8.15 .1)$. If $\left(k^{\prime}, m^{\prime}\right)=(0,3)$, then we have a $(1,4,3)$-monomializing $\ell$ basis $u_{2}^{\prime}, v^{\prime}=\mu^{\prime} x_{1}^{\prime} u_{1}^{\prime}+u_{2}^{\prime 3}, u_{1}^{\prime}$ of the first kind for $I \supset H$ at $P^{\prime}$ (8.15.2), where we note $\left.x_{1}^{\prime}\right|_{C^{b}}=t^{\prime}$ by $a_{1}^{\prime}=1(9.1)$.

Thus we have by (8.12)

$$
\begin{aligned}
0 \leq & l \operatorname{deg}_{C} \mathscr{M}+\frac{1}{4} l \operatorname{deg}_{C} \mathscr{L}+\left(\frac{1}{2}-\frac{1}{4}\right) \frac{1}{m}\left(m-a_{2}-2\right)+\varepsilon^{\prime}\left(\frac{1}{3}-\frac{1}{4}\right) \frac{1}{m^{\prime}} \\
& \left(\text { resp. } 0 \leq l \operatorname{deg}_{C} \mathscr{M}+\frac{1}{4} l \operatorname{deg}_{C} \mathscr{L}+\left(\frac{1}{3}-\frac{1}{4}\right) \frac{1}{m}\left(m-a_{2}-2\right)+\varepsilon^{\prime}\left(\frac{1}{3}-\frac{1}{4}\right) \frac{1}{m^{\prime}}\right),
\end{aligned}
$$

where $\varepsilon^{\prime}=1$ or 0 according as $\left(k^{\prime}, m^{\prime}\right)=(0,3)$ or otherwise.

If $m^{\prime} \geq 4$, then we have $\varepsilon^{\prime}=0$, and in either case, we get

$$
0 \leq\left(\frac{1}{m}-\frac{1}{m^{\prime}}\right)+\frac{1}{4}\left(\frac{m-a_{2}}{m}+\frac{1}{m^{\prime}}\right)+\frac{m-a_{2}-2}{4 m},
$$


whence $m \leq\left(2 m-2 a_{2}+2\right) m^{\prime} / 3$. Using (9.3.4), one sees

$$
\left(m-a_{2}\right) m^{\prime}<m \leq\left(2 m-2 a_{2}+2\right) m^{\prime} / 3
$$

whence $m-a_{2}<\left(2 m-2 a_{2}+2\right) / 3$, i.e. $m-a_{2}<2$. This implies $m-a_{2}=1$, which contradicts $a_{2} \not \equiv \pm 1(m)$.

If $m^{\prime}=3$, then in either case, we get

$$
0 \leq\left(\frac{1}{m}-\frac{1}{3}\right)+\frac{1}{4}\left(\frac{m-a_{2}}{m}+\frac{1}{3}\right)+\frac{m-a_{2}-2}{4 m}+\frac{1}{36},
$$

whence $m \leq 9\left(m-a_{2}+1\right) / 4$. Thus by (9.3.4), we have

$$
3\left(m-a_{2}\right)<m \leq 9\left(m-a_{2}+1\right) / 4,
$$

and $4\left(m-a_{2}\right)<3\left(m-a_{2}+1\right)$, i.e. $m-a_{2}<3$. Thus $m-a_{2}=2$ by $a_{2} \not \equiv \pm 1$ $(m)$. Then one has $6<m \leq 27 / 4=6.75$, which is a contradiction. Thus (9.3.b) does not occur.

(9.3.8) Case (9.3.c). Our purpose here is to prove $a_{1}=a_{1}^{\prime}=1$ and smoothness of $\left(C^{\sharp}, P^{\sharp}\right)$ and $\left(C^{b}, P^{b}\right)$. By symmetry of $P$ and $P^{\prime}$, we may assume $a_{2}^{\prime} / m^{\prime}>$ $1 / 2$ in view of (9.3.4). Since $\operatorname{siz}_{P^{\prime}}=1$, we have $m^{\prime} \geq a_{1}^{\prime} a_{2}^{\prime}>a_{1}^{\prime} m^{\prime} / 2$. Thus $a_{1}^{\prime}=1$. We will prove $a_{1}=1$ by deriving a contradiction assuming $a_{1} \geq 2$ till end of (9.3.8.8). We have $q l_{C}(\mathscr{L})=a_{1} P^{\sharp}+P^{b}$ and $q l_{C}(\mathscr{M})=$ $-1+\left(m-a_{1} a_{2}\right) P^{\sharp}+\left(m^{\prime}-a_{2}^{\prime}\right) P^{b}$. Thus (9.3.3) reduces to

$$
0 \leq\left(-1+\frac{m-a_{1} a_{2}}{m}+\frac{m^{\prime}-a_{2}^{\prime}}{m^{\prime}}\right)+\frac{1}{2}\left(\frac{a_{1}}{m}+\frac{1}{m^{\prime}}\right) .
$$

Using (9.3.4), we rewrite it as

$$
0<-\frac{a_{1} a_{2}}{m}+\frac{a_{2}}{m}+\frac{1}{2}\left(\frac{a_{1}}{m}+\frac{1}{m^{\prime}}\right)
$$

that is

$$
\frac{1}{m}\left(2 a_{1} a_{2}-2 a_{2}-a_{1}\right)<\frac{1}{m^{\prime}} \text {. }
$$

(9.3.8.3) Claim $a_{2}=1$. If $a_{2} \geq 2$, then

$$
\left(2 a_{1} a_{2}-2 a_{2}-a_{1}\right)-a_{2}=\left(2 a_{1}-3\right) a_{2}-a_{1} \geq 4 a_{1}-6-a_{1} \geq 0,
$$

whence we have $1 / m^{\prime}>a_{2} / m$. Then (9.3.4) implies $1<a_{2} / m+a_{2}^{\prime} / m^{\prime}<$ $\left(1+a_{2}^{\prime}\right) / m^{\prime} \leq 1$, which is a contradiction and $a_{2}=1$ is proved.

(9.3.8.4) Claim $a_{1}=2$. By $a_{2}=1,(9.3 .4)$ implies $1 / m>\left(m^{\prime}-a_{2}^{\prime}\right) / m^{\prime} \geq$ $1 / m^{\prime}$. (9.3.8.2) implies $1 / m^{\prime}>\left(a_{1}-2\right) / m$, whence $1>a_{1}-2$. By the hypothesis $a_{1} \geq 2$, we have $a_{1}=2$.

(9.3.8.5) Since $a_{2}=1$ and $a_{1}^{\prime}=1,\left(C^{\sharp}, P^{\sharp}\right)$ and $\left(C^{b}, P^{b}\right)$ are smooth, whence

$$
U(z)=\lceil z / m\rceil \text { and } U^{\prime}(z)=\left\lceil z / m^{\prime}\right\rceil \quad(z \in \mathbb{Z}),
$$


and

$$
T L\left(z+z^{\sharp} P^{\sharp}+z^{b} P^{b}\right)=z+\left[z^{\sharp} / m\right]+\left[z^{b} / m^{\prime}\right] .
$$

One also sees $q l_{C}(\mathscr{L})=2 P^{\sharp}+P^{b}, q l_{C}(\mathscr{M})=-1+(m-2) P^{\sharp}+\left(m^{\prime}-a_{2}^{\prime}\right) P^{b}$, and $q l_{C}\left(\mathrm{gr}_{C}^{0} \omega\right)=-1+(m-1) P^{\sharp}+\left(m^{\prime}-a_{2}^{\prime}\right) P^{b} \quad(2.10)$ and (8.9.1).

(9.3.8.6) Claim $m \geq 5$. Since $a_{1}=2$ is prime to $m$ and $m>1$, it is enough to derive a contradiction assuming $m=3$. Then (9.3.4) implies $3\left(m^{\prime}-\right.$ $\left.a_{2}^{\prime}\right)<m^{\prime}$. Thus by (9.3.8.5) and the additivity of $q l_{C}$, we can calculate the values of $q l_{C}$ for subquotients $\omega \widetilde{\otimes} \mathscr{L}^{\widetilde{\otimes} i} \widetilde{\otimes} \mathscr{M}^{\widetilde{\otimes}^{j}}(i, j \geq 0,4>2 i+j>0)$ of $F^{1}(\omega, J) / F^{4}(\omega, J)$ as follows:

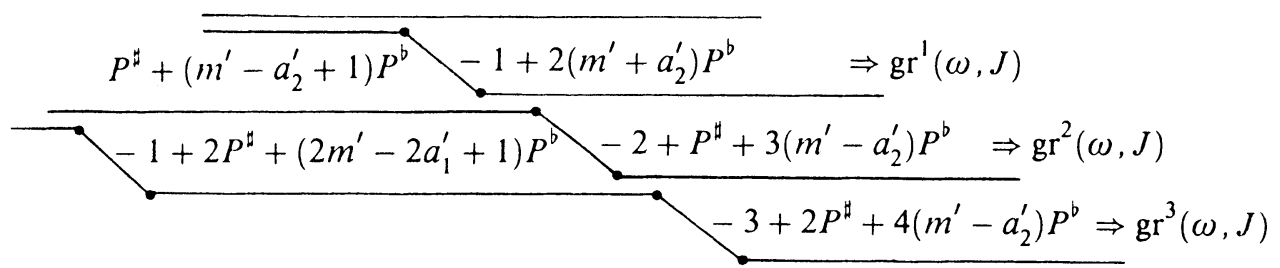

as in (9.3.7.5). The expressions are normalized except for $q l_{C}\left(\omega \widetilde{\otimes} \mathscr{M}^{\widetilde{\otimes} 3}\right)=$ $-3+2 P^{\sharp}+4\left(m^{\prime}-a_{2}^{\prime}\right) P^{b}$; it is not normalized only when $4\left(m^{\prime}-a_{2}^{\prime}\right) \geq m^{\prime}$ and its normalized form in this case is $-2+2 P^{\sharp}+\left(3 m^{\prime}-4 a_{2}^{\prime}\right) P^{b}$. Thus $\chi\left(F^{1}(\omega, J) / F^{4}(\omega, J)\right)=-1$ or -2 , which contradicts (8.7). Hence $m \geq 5$.

(9.3.8.7) By $m \geq 5$, we see $q l_{C}\left(\mathscr{M}^{\widetilde{\otimes} 2}\right)=-1+(m-4) P^{\sharp}+2\left(m^{\prime}-a_{2}^{\prime}\right) P^{b}$ and $\operatorname{deg}_{C} \mathscr{M}^{\widetilde{\otimes} 2}=-1$. Thus

$$
0 \rightarrow \mathscr{M}^{\widetilde{\otimes} 2} \rightarrow \mathrm{gr}_{C}^{0} J \rightarrow \mathscr{L} \rightarrow 0
$$

splits as $\mathscr{O}_{C}$-modules and $\operatorname{gr}_{C}^{0} J$ contains unique submodules isomorphic to $\mathscr{L}$, whence we write as $\operatorname{gr}_{C}^{0} J=\mathscr{M}^{\widetilde{\otimes} 2} \oplus \mathscr{L}_{J}$.

Let $K$ be the $C$-primary ideal such that $J \supset K \supset F^{1} J$ and $K / F^{1} J=$ $\mathscr{L}_{J}$. Then $K$ is a $C$-laminal ideal of width 3 which is a nested c.i. outside $\left\{P, P^{\prime}\right\}$, and we have $\ell$-isomorphisms $\operatorname{Ker} \beta_{K}=\operatorname{Ker} \beta_{J}=\mathscr{L}$ and $\operatorname{gr}^{1}(\mathscr{O}, K)=$ $\operatorname{gr}^{1}(\mathscr{O}, J) \simeq \mathscr{M}$ by (8.14). We see that $\operatorname{gr}_{C}^{0} J=\mathscr{L}_{J} \oplus \mathscr{M}^{\widetilde{\otimes} 2}$ is an $\ell$-splitting at $P^{\prime}(9.1 .8)$ because $q l \operatorname{deg}\left(\mathscr{L}, P^{\prime}\right)=1<q l \operatorname{deg}\left(\mathscr{M}^{\widetilde{\otimes} 2}, P^{\prime}\right)=2\left(m^{\prime}-a_{2}^{\prime}\right)$ by $m\left(m^{\prime}-a_{2}^{\prime}\right)<m^{\prime}$ (9.3.4). Hence the $(1,2)$-monomializing $\ell$-basis of $I \supset J$ at $P^{\prime}$ lifts to a $(1,3)$-monomializing $\ell$-basis of $I \supset K$ at $P^{\prime}$ (8.15). Let

$$
\left(\mathscr{L}_{J}\right)_{P}=\mathscr{O}_{C, P}\left(\mu t^{2} u_{1}+t^{m k+m-4} u_{2}{ }^{2}\right) \text {, }
$$

where $\mu$ is a unit and $0 \leq k \leq \infty$. If $(k, m)=(0,5) \quad($ resp. $(k, m) \neq(0.5))$, then $u_{2}, v=\mu x_{2} u_{1}+u_{2}^{2}, u_{1}$ form a $(1,3,2)$-monomializing $\ell$-basis of the 
first kind with attached semi-invariant $\mu x_{2}$ (resp. $\left(u_{2}, u_{1}\right)$ lifts to a $(1,3)$ monomializing $\ell$-basis) of $I \supset K$ at $P(8.15)$, where we note $\left.x_{2}\right|_{C^{\sharp}}=t$ by $a_{2}=1$ (9.1). Thus by $(8.12(\mathrm{ii}))$ and $(8.11(\mathrm{iii}))$, we have

$$
\begin{aligned}
0 & \leq l \operatorname{deg}_{C} \mathscr{M}+\frac{1}{3} l \operatorname{deg}_{C} \mathscr{L}+\left(\frac{1}{2}-\frac{1}{3}\right) \frac{\varepsilon}{m} \\
& =\left(-1+\frac{m-2}{m}+\frac{m^{\prime}-a_{2}^{\prime}}{m^{\prime}}\right)+\frac{1}{3}\left(\frac{2}{m}+\frac{1}{m^{\prime}}\right)+\frac{\varepsilon}{6 m},
\end{aligned}
$$

where $\varepsilon=1$ (resp. $\varepsilon=0)$. Combining this with $\left(m^{\prime}-a_{2}^{\prime}\right) m<m^{\prime}$ (9.3.4), we have

$$
(8-\varepsilon)\left(m^{\prime}-a_{2}^{\prime}\right) m<(8-\varepsilon) m^{\prime} \leq m\left\{6\left(m^{\prime}-a_{2}^{\prime}\right)+2\right\} .
$$

Thus $(8-\varepsilon)\left(m^{\prime}-a_{2}^{\prime}\right)<6\left(m^{\prime}-a_{2}^{\prime}\right)+2$, whence $m^{\prime}-a_{2}^{\prime}=\varepsilon=1$ because $m^{\prime}-a_{2}^{\prime} \geq 1$ and $\varepsilon=0,1$. Hence $m=5$ by $\varepsilon=1$. Then (9.3.8.8) implies $7 \cdot 5<7 \cdot m^{\prime} \leq 5 \cdot 8$, which is impossible. Thus we have shown $a_{1}=a_{1}^{\prime}=1$ in case (9.3.c), and we are done.

(9.4) Theorem. Let the notation and assumptions be as in (9.1). Then $P$ and $P^{\prime}$ are type (IA) points.

Proof. It is enough to derive a contradiction assuming that $P$ is of type (IIA) or $\left(\right.$ IA $\left.^{\vee}\right)$. Indeed it would imply that $P$ is of type (IA) and hence $P^{\prime}$ is also of type (IA) by symmetry of $P$ and $P^{\prime}$. Before we start the calculation leading to a contradiction, we will replace $X \supset C$ with some nearby extremal nbd since it is enough to disprove the latter.

(9.4.1) By replacing $X \supset C$ with its $L$-deformation at $P^{\prime}$ (4.7), we may assume that $P^{\prime}$ is ordinary (hence of type (IA)). Then $\left(C^{b}, P^{b}\right)$ is smooth and $a_{1}^{\prime}=1$ modulo necessary permutations as in (4.2). Indeed a further $L$-deformation of $X \supset C$ at $P$ would have only ordinary points, whence $\left(C^{b}, P^{b}\right)$ is smooth and $a_{1}^{\prime}=1$ modulo permutations by (9.3).

Then $P$ is a type (IIA) point. Indeed if it is of type $\left(\mathrm{IA}^{\vee}\right)$, then replacing $X \supset C$ with its $L$-deformation (4.7) at $P$, we have an extremal nbd $X \supset$ $C \simeq \mathbb{P}^{1}$ with ordinary $P, P^{\prime}$ of indices $\geq 3$ such that $P$ is imprimitive, which contradicts $(9.3)$.

(9.4.2) By replacing $X \supset C$ with its $L^{\prime}$-deformation at $P(4.12 .2($ ii) ), we may take an $\ell$-coordinate system $y=\left(y_{1}, \ldots, y_{4}\right)$ and an $\ell$-equation $\psi$ for $(X, P)$ such that wt $y_{i} \equiv a_{i} \quad(i \in[1,4]), C^{\sharp}$ is the $y_{1}$-axis, $\phi=y_{1} y_{2}+y_{3}{ }^{2}+y_{4}{ }^{3}$ (we note that there exists no singularity other than $P, P^{\prime}$ by $\left.(6.2)\right)$. Hence $i_{P}(1)=1$ by (2.16). Thus by $i_{P^{\prime}}(1)=\operatorname{siz}_{P^{\prime}}=1$, we have $\operatorname{deg} \operatorname{gr}_{C}^{1} \mathscr{O}=-1$ (2.3.2) and $\operatorname{gr}_{C}^{1} \mathscr{O} \simeq \mathscr{O} \oplus \mathscr{O}(-1)$. Then $\mathscr{L} \subset \operatorname{gr}_{C}^{1} \mathscr{O}$ such that $\mathscr{L} \simeq \mathscr{O}$ is unique, and $\mathscr{L}$ is an $\ell$-invertible $\mathscr{O}_{C}$-module (9.1.7).

We note that $y_{2} \in I^{\sharp(2)}$, and $\left\{y_{3}, y_{4}\right\}$ is an $\ell$-free $\ell$-basis of $\operatorname{gr}_{C}^{1} \mathscr{O}$ at $P$ such that $R\left(\right.$ wt $\left.y_{3}\right)=R\left(a_{3}\right)=1$ and $R\left(\right.$ wt $\left.y_{4}\right)=R\left(a_{4}\right)=2$ (4.2). Let 
$y_{1}\left(f_{3} y_{3}+f_{4} y_{4}\right) \quad\left(f_{3}, f_{4} \in \mathscr{O}_{C^{\sharp}, P \sharp}\right)$ be a basis of $\mathscr{L}$ at $P$. Then $q l \operatorname{deg}(\mathscr{L}, P)=1$ if $f_{3}$ is a unit ( 2 if otherwise) by (8.8.5).

(9.4.3) We consider the case $q l \operatorname{deg}(\mathscr{L}, P) \neq 1$ (otherwise this process is not needed). In this case, we see ord $f_{4}=1$ as above. Let $X_{\lambda} \supset C_{\lambda}$ be the twisted extensions (1b.8.1) of the trivial deformation

$$
(X, P) \supset(C, P) \quad \text { by }\left(y_{1}\left(f_{3} y_{3}+f_{4} y_{4}+\lambda y_{3}\right), y_{1} y_{3}\right) \text {. }
$$

Then $\operatorname{deg} \operatorname{gr}_{C_{\lambda}}^{1} \mathscr{O}=-1$ by (2.3.2) since the deformation is locally trivial. Then $I^{(2)}$ deforms as $I_{\lambda}^{(2)}$ by (1b.8.3(i)). Since $\mathscr{L}_{P}$ is generated by $y_{1}\left(f_{3} y_{3}+f_{4} y_{4}\right)$, $\mathscr{L}$ deforms as an invertible subsheaf $\mathscr{L}_{\lambda}$ of $I_{\lambda} / I_{\lambda}^{(2)}$ such that $\mathscr{L}_{\lambda}$ is generated by $y_{1}\left(\left(f_{3}+\lambda\right) y_{3}+f_{4} y_{4}\right)$ at $P_{\lambda}(=P)$ and $\mathscr{L}_{\lambda}$ is the trivial deformation of $\mathscr{L}$ in $X_{\lambda, \text { out }}=X_{0, \text { out }}(1 \mathrm{~b} .8 .3(\mathrm{ii}))$. Then replacing $X \supset C$ by nearby extremal nbd $X_{\lambda}^{\circ} \supset C_{\lambda}$, we may assume $q l \operatorname{deg}(\mathscr{L}, P)=1$, anyway.

(9.4.4) Thus we may assume $q l \operatorname{deg}(\mathscr{L}, P)=1$. We also see $q l \operatorname{deg}\left(\mathscr{L}, P^{\prime}\right)=1$ or $R^{\prime}\left(a_{2}^{\prime}\right)$ by $(9.3 .1)$ and $(8.8 .5)$ because $a_{1}^{\prime}=1(9.4 .1)$. Hence if $q l \operatorname{deg}\left(\mathscr{L}, P^{\prime}\right)$ $>1$, then we can apply a process similar to $(9.4 .3)$, and thus we may also assume $q l \operatorname{deg}\left(\mathscr{L}, P^{\prime}\right)=1$.

(9.4.5) Since we have $\operatorname{deg} \operatorname{gr}_{C}^{1} \mathscr{O}=-1$ and $\operatorname{gr}_{C}^{1} \mathscr{O} \supset \mathscr{L} \simeq \mathscr{O}$, we have a splitting $\operatorname{gr}_{C}^{1} \mathscr{O}=\mathscr{L} \oplus \mathscr{O}(-1)$ and hence an $\ell$-splitting $\operatorname{gr}_{C}^{1} \mathscr{O}=\mathscr{L} \widetilde{\oplus} \mathscr{M}$ for some submodule $\mathscr{M} \simeq \mathscr{O}(-1)$ (9.1.7(iii)). By (9.4.4), we have $q l_{C}(\mathscr{L})=P^{\sharp}+P^{b}$ and $q l_{C}(\mathscr{M})=-1+2 P^{\sharp}+\left(m^{\prime}-a_{2}^{\prime}\right) P^{b} \quad(8.8 .5)$ and (9.4.2). Since process (9.4.3) and (9.4.4) does not change $(X, P) \supset(C, P)$, we may use $(y)$ given in (9.4.2). Since $q l \operatorname{deg}(\mathscr{L}, P)=1, \mathscr{L}$ has an $\ell$-free $\ell$-basis $y_{3}+f y_{1} y_{4}$ at $P$ (9.4.2), where $f$ is an invariant in $\mathbb{C}\{y\}$. If we set $y_{3}^{\prime \prime}=y_{3}+f y_{1} y_{4}$ and $y_{2}^{\prime \prime}=y_{2}-2 f y_{3} y_{4}-f^{2} y_{1} y_{4}{ }^{2}$, then $\left(y_{1}, y_{2}^{\prime \prime}, y_{3}^{\prime \prime}, y_{4}\right)$ is an $\ell$-coordinate system with similar properties (e.g. $\psi=y_{1} y_{2}^{\prime \prime}+y_{3}^{\prime \prime 2}+y_{4}{ }^{3}$ ). Thus choosing $\left(y_{1}, y_{2}^{\prime \prime}, y_{3}^{\prime \prime}, y_{4}\right)$ as new $\left(y_{1}, y_{2}, y_{3}, y_{4}\right)$, we may simply assume that $y_{3}$ is an $\ell$-free $\ell$-basis of $\mathscr{L}$ at $P$.

(9.4.6) Let $J$ be a $C$-laminal ideal of width 2 such that $J / I^{(2)}=\mathscr{L}$. Then as in (9.3.2), $J$ is a nested c.i. outside $\left\{P, P^{\prime}\right\}$ and we have equalities $\operatorname{Im} \alpha_{J}=$ $\operatorname{Ker} \beta_{J}=\mathscr{L}$ with $\ell$-structure and an $\ell$-isomorphism $\operatorname{gr}^{1}(\mathscr{O}, J) \simeq \mathscr{M}$. Also as in (9.3.2), we can see that $J$ is $(1,2)$-monomializable at ordinary point $P^{\prime}$ by the $\ell$-splitting $\operatorname{gr}_{C}^{0} I=\operatorname{Im} \alpha_{J} \widetilde{\oplus} \mathscr{M}$. Then (9.4.5) shows that $\left(y_{4}, y_{3}, y_{2}\right)$ is a $(1,2,3)$-monomializing $\ell$-basis of the second kind for $I \supset J$ at $P$. Let $\mathscr{D}$ be the $\ell$-invertible $\mathscr{O}_{C}$-module defined in $\left(8.11 .1\right.$ (iii)) from $\left(y_{4}, y_{3}, y_{2}\right)$, then we have a normalized expression $q l_{C} \mathscr{D}=P^{\sharp}$. We now have an $\ell$-isomorphism

$$
\mathrm{gr}^{n, i}(\omega, J) \simeq \mathscr{M}^{\widetilde{\otimes}(2 i+r)} \tilde{\otimes} \mathscr{D}^{\widetilde{\otimes}[(2 i+r) / 3]} \tilde{\otimes} \mathscr{L}^{\widetilde{\otimes}(q-i)} \tilde{\otimes} \mathrm{gr}_{C}^{0} \omega
$$

for all $n=2 q+r \geq 0$ with $r \in[0,1]$ and $i \in[0, q]$ by (8.12) (with $d=2$, $\left.t=2, s=1, s^{\prime}=0\right)$. Now the way we derive a contradiction is very similar to 
(9.3.8.6). We note $q l_{C}\left(\mathrm{gr}_{C}^{0} \omega\right)=-1+3 P^{\sharp}+\left(m^{\prime}-a_{2}^{\prime}\right) P^{\mathrm{b}}(2.10)$ and (8.9.1), and calculate the values of $q l_{C}$ for subquotients $\operatorname{gr}^{n, i}(\omega, J) \quad(0 \leq i \leq[n / 2], n \in$ $[1,3])$ of $F^{1}(\omega, J) / F^{4}(\omega, J)$ as in (9.3.8.6) by (9.4.5) and additivity of $q l_{C}$. We get

$$
\begin{aligned}
q l_{C}\left(\mathrm{gr}^{1,0}(\omega, J)\right) & =q l_{C}\left(\mathscr{M} \widetilde{\otimes} \operatorname{gr}_{C}^{0} \omega\right) \\
& =q l_{C}(\mathscr{M})+q l_{C}\left(\mathrm{gr}_{C}^{0} \omega\right) \\
& =-1+P^{\sharp}+2\left(m^{\prime}-a_{2}^{\prime}\right) P^{b}, \\
q l_{C}\left(\mathrm{gr}^{2,0}(\omega, J)\right) & =q l_{C}\left(\mathscr{L} \widetilde{\otimes} \operatorname{gr}_{C}^{0} \omega\right) \\
& =\left(m^{\prime}-a_{2}^{\prime}+1\right) P^{b}, \\
q l_{C}\left(\operatorname{gr}^{2,1}(\omega, J)\right) & =q l_{C}\left(\mathscr{M}^{\widetilde{\otimes} 2} \tilde{\otimes} \operatorname{gr}_{C}^{0} \omega\right) \\
& =-2+3 P^{\sharp}+3\left(m^{\prime}-a_{2}^{\prime}\right) P^{b}, \\
q l_{C}\left(\operatorname{gr}^{3,0}(\omega, J)\right) & =q l_{C}\left(\mathscr{M} \widetilde{\otimes} \mathscr{L} \widetilde{\otimes} \operatorname{gr}_{C}^{0} \omega\right) \\
& =-1+2 P^{\sharp}+\left\{2\left(m^{\prime}-a_{2}^{\prime}\right)+1\right\} P^{b}, \\
q l_{C}\left(\operatorname{gr}^{3,1}(\omega, J)\right) & =q l_{C}\left(\mathscr{M}^{\widetilde{\otimes} 3} \widetilde{\otimes} \mathscr{D} \widetilde{\otimes} \operatorname{gr}_{C}^{0} \omega\right) \\
& =-2+2 P^{\sharp}+4\left(m^{\prime}-a_{2}^{\prime}\right) P^{b},
\end{aligned}
$$

which are arranged as

$$
\longrightarrow \frac{-1+P^{\sharp}+2\left(m^{\prime}-a_{2}^{\prime}\right) P^{b} \Rightarrow g^{1}(\omega, J)}{\left(1+m^{\prime}-a_{2}^{\prime}\right) P^{b}} \Rightarrow g^{2}(\omega, J)
$$

The expressions are normalized $(8.9 .1)$ because $4\left(m^{\prime}-a_{2}^{\prime}\right)<m^{\prime}$ by $w_{P^{\prime}}(0)=$ $\left(m^{\prime}-a_{2}^{\prime}\right) / m^{\prime}<1-w_{P}(0)=1 / 4(2.3 .3)$ and $(4.9(\mathrm{i}))$. Thus

$$
\chi\left(F^{1}(\omega, J) / F^{4}(\omega, J)\right)<0
$$

(8.9.1(iii)), which contradicts $H^{1}(\omega)=0$ as in (8.7) and we are done.

(9.4.7) Corollary-Definition. Points $P$ and $P^{\prime}$ are type (IA) points such that $a_{1}=a_{1}^{\prime}=1$ (after necessary permutations are made as in (4.2)), and $\ell(P)<m$ and $\ell\left(P^{\prime}\right)<m^{\prime}$, where we define $\ell(P)=\operatorname{len}_{P \sharp} I^{\sharp(2)} / I^{\sharp 2}$ and $\ell\left(P^{\prime}\right)=$ $\operatorname{len}_{P^{b}} I^{b(2)} / I^{b 2}$.

We note that $P$ is ordinary iff $\ell(P)=0$.

Proof. By symmetry between $P$ and $P^{\prime}$, we need to check the assertions only for $P$ and $P^{\sharp}$. We will derive a contradiction assuming that $a_{1}=1$ cannot be 
achieved, that is either (i) $1<a_{1}<m-1$, or (ii) $a_{1}=m-1$ and $a_{2}>1$. Since it is enough to disprove nearby extremal nbds, we may replace $X \supset C$ with some nearby extremal nbd. So we may replace $X \supset C$ with an $L$-deformation $Y \supset D$ at $P(4.7)$, and further with an $L$-deformation at $P^{\prime}$ of $Y \supset D$. In this process $a_{i}$ 's are not changed since $P$ and $P^{\prime}$ are of type (IA). Thus our $X \supset C$ has two ordinary points $P, P^{\prime}$ with indices $m, m^{\prime} \geq 3$ such that (i) $1<a_{1}<m-1$, or (ii) $a_{1}=m-1$ and $a_{2}>1$. This contradicts (9.3). Thus $a_{1}=1$ and similarly $a_{1}^{\prime}=1$ (modulo necessary permutations). If $\ell(P) \geq m$, then an $L^{\prime}$-deformation of $X \supset C$ at $P(4.12 .2)$ is locally primitive and has at least one point of type (III) besides $P$ and $P^{\prime}$ of indices $m, m^{\prime} \geq 3$. This contradicts (6.2), and hence $\ell(P)<m$. Thus we are done.

(9.4.8) Corollary. We have $i_{P}(1)=i_{P^{\prime}}(1)=1$ and an isomorphism $\operatorname{gr}_{C}^{1} \mathscr{O} \simeq$ $\mathscr{O} \oplus \mathscr{O}(-1)$.

Proof. By (2.16), we have $i_{P}(1)=i_{P^{\prime}}(1)=1$. Thus $\operatorname{deg} \operatorname{gr}_{C}^{1} \mathscr{O}=-1$ by (2.3.2). Hence we have $\operatorname{gr}_{C}^{1} \mathscr{O} \simeq \mathscr{O} \oplus \mathscr{O}(-1)$ by $H^{1}\left(\operatorname{gr}_{C}^{1} \mathscr{O}\right)=0$ (2.3.4).

(9.5) Let the notation and assumptions be as in (9.1). Then $P$ and $P^{\prime}$ are of type (IA) and we may assume $a_{1}=a_{1}^{\prime}=1$. Then $\operatorname{gr}_{C}^{1} \mathscr{O} \simeq \mathscr{O} \oplus \mathscr{O}(-1)$, hence there exists exactly one submodule $\mathscr{L}$ of $\operatorname{gr}_{C}^{1} \mathscr{O}$ such that $\mathscr{L} \simeq \mathscr{O}_{C}$, and it is $\operatorname{gr}_{C}^{1} \mathscr{O}$-saturated. We note by $(9.1 .7(\mathrm{ii})), \mathscr{L}$ is an $\ell$-invertible $\mathscr{O}_{C}$-module.

Though the following is formulated only for $P$, it also applies to $P^{\prime}$ modulo obvious changes.

(9.5.1) Definition-Lemma. Let $q(P)=q l \operatorname{deg}(\mathscr{L}, P)$. Then there exists $\sigma \in \mathfrak{S}_{4}$ with $\sigma(1)=1$ such that $\ell(P)=R\left(a_{\sigma(4)}\right), q(P)=R\left(a_{\sigma(3)}\right)$, and $\left\{y_{\sigma(2)}, y_{\sigma(3)}\right\}$ is an $\ell$-free $\ell$-basis of $\operatorname{gr}_{C}^{1} \mathscr{O}$ at $P$.

Proof. There exists an $\ell$-coordinate system $(y)=\left(y_{1}, \ldots, y_{4}\right)$ and an $\ell$-equation $\phi$ at $P$ such that $C^{\sharp}=y_{1}$-axis, wt $y_{i} \equiv a_{i} \quad(i \in[1,4])$, and $\phi \equiv y_{1}^{\ell(P)} y_{\sigma(4)} \bmod \left(y_{2}, y_{3}, y_{4}\right)^{2}$ for some $\sigma \in \mathfrak{S}_{4}$ such that $\sigma(1)=1$ (2.16). Then $\ell(P)=R\left(a_{\sigma(4)}\right)$ by $\ell(P)<m(2.16)$, and $\left\{y_{\sigma(2)}, y_{\sigma(3)}\right\}$ is an $\ell$-free $\ell$-basis of $\operatorname{gr}_{C}^{1} \mathscr{O}$ at $P$. Thus $q(P)=R\left(a_{\sigma(2)}\right)$ or $R\left(a_{\sigma(3)}\right)$.

We will define deformation processes of $X \supset C$ which are more delicate than the ones in (4.7). We begin with a general description.

(9.6) Let the notation and assumptions be as in (9.5). We will choose an $\ell$-coordinate system $(y)=\left(y_{1}, \ldots, y_{4}\right)$ and an $\ell$-equation $\phi$ at $P$ such that $C^{\sharp}=y_{1}$-axis and wt $y_{i} \equiv a_{i}(m) \quad(i \in[1,4])$, and invariant $u_{1}, u_{2} \in$ $\left(y_{2}, y_{3}, y_{4}\right) \mathbb{C}\{y\}$ such that $\operatorname{gr}_{C}^{1} \mathscr{O} /\left(u_{1}, u_{2}\right)$ is of finite length and $\mathscr{L}$ is generated by $u_{1}$ at $P$. We will choose an invariant $\psi \in\left(y_{2}, y_{3}, y_{4}\right) \mathbb{C}\{y\}$ such that

$$
\text { g.c.d. }\{\phi, \psi\}=1 \text { and } \operatorname{Sing}\{\phi=\psi=0\} \cap y_{4} \text {-axis } \subset\{0\} \text {, }
$$


and

$$
\left(X_{\lambda}, P_{\lambda}\right) \text { is terminal if } 0<|\lambda| \ll 1 \text {, }
$$

where $\phi_{\lambda}=\phi+\lambda \psi$, and $\left(X_{\lambda}, P_{\lambda}\right)=\left(\left\{\phi_{\lambda}=0\right\}, 0\right) / \mu_{m} \supset\left(C_{\lambda}, P_{\lambda}\right)=$ $\left(y_{1}\right.$-axis, 0$) / \boldsymbol{\mu}_{m}(\lambda \in \mathbb{C})$. We note

(9.6.3) Remark. ( $y)$ is an $\ell$-coordinate system for $X_{\lambda} \supset C$ at $P_{\lambda}=P$, whence $\left(a_{1}, \ldots, a_{4}\right)$ remain the same for $P_{\lambda}$.

Then we can apply (1b.3) as in step (L) in $\S 1 \mathrm{~b}$, and following step (LG) in $\S 1 \mathrm{~b}$, let $X_{\lambda} \supset C_{\lambda}$ be the twisted extension of $\left(X_{\lambda}, P_{\lambda}\right) \supset\left(C_{\lambda}, P_{\lambda}\right)$ by $u=\left(u_{1}, u_{2}\right)$. We note that $X_{\lambda} \supset C_{\lambda}(=C)$ may be identified with $X \supset C$ outside a small nbd of $P_{\lambda}$ :

$$
X_{\lambda, \text { out }}=X-X\left(\begin{array}{c}
{[0, \beta] \times \gamma} \\
u<\delta
\end{array}\right) \supset C_{\lambda, \text { out }}=C \cap X_{\lambda, \text { out }},
$$

where $\beta, \gamma, \delta \in \mathbb{C}$ are as in (1b.8.1). Then by step $(\mathrm{G})$ in $\S 1 \mathrm{~b}$, we have nearby extremal nbds $X_{\lambda}^{\circ} \supset C_{\lambda}=C$, where $X_{\lambda}^{\circ}$ is a nbd of $C_{\lambda}$ in $X_{\lambda}$. Though we have not yet explicitly constructed $X_{\lambda}^{\circ} \supset C_{\lambda}$, we can say the following.

(9.6.4) Lemma. For an arbitrary small enough $\lambda, X_{\lambda}^{\circ} \supset C_{\lambda}$ is a locally primitive extremal nbd with exactly two singular points $P_{\lambda}$ and $P_{\lambda}^{\prime}$, which are of type (IA) and have indices $m$ and $m^{\prime}$. Let $I_{\lambda}$ be the sheaf of defining ideals of $C_{\lambda}$ in $X_{\lambda}$, then the coherent sheaves $\operatorname{gr}^{1}\left(\mathscr{O}, I_{\lambda}\right)$ on $C_{\lambda}$ form a flat family with parameter $\lambda$, and $\mathscr{L}$ deforms as submodules $\mathscr{L}_{\lambda}(\simeq \mathscr{O})$ of $\operatorname{gr}^{1}\left(\mathscr{O}, I_{\lambda}\right)$ such that

$$
\left.\mathscr{L}_{\lambda}\right|_{C_{\lambda, \text { out }}}=\left.\mathscr{L}\right|_{C_{0, \text { out }}} \text { and } \mathscr{L}_{\lambda} \otimes \mathscr{O}_{C_{\lambda}, P_{\lambda}}=\mathscr{O}_{C_{\lambda}, P_{\lambda}} u_{1} .
$$

In particular we have $\ell\left(P_{\lambda}^{\prime}\right)=\ell\left(P^{\prime}\right)$ and $q\left(P_{\lambda}^{\prime}\right)=q\left(P^{\prime}\right)$.

Proof. By smoothness of $\left(C^{\sharp}, P^{\sharp}\right)$, we have local primitiveness of $X_{\lambda} \supset C_{\lambda}$ in a nbd of $P_{\lambda}$ (1b.3.1), whence local primitiveness of $X_{\lambda} \supset C_{\lambda}$ follows by the definition of twisted extension. It is clear that $X_{\lambda}^{\circ} \supset C_{\lambda}$ has two singular points $P_{\lambda}$ and $P_{\lambda}^{\prime}$ with indices $m, m^{\prime}(\geq 3)$, whence $X_{\lambda}^{\circ}$ has no other singular points on $C_{\lambda}$ by (6.2). They are of type (IA) by (9.6.3) and triviality of deformation near $P^{\prime}$. Thus deg $\operatorname{gr}^{1}\left(\mathscr{O}, I_{\lambda}\right)=-1$ by $(9.5)$, and $\operatorname{gr}^{1}\left(\mathscr{O}, I_{\lambda}\right)$ 's form a flat family by (1b.8.3(i)). The assertions on $\mathscr{L}$ follow from (1b.8.3(ii)). We see that $\ell\left(P_{\lambda}^{\prime}\right)=\ell\left(P^{\prime}\right)$ by $X_{\lambda, \text { out }}{ }^{\text {" }}=" X_{0, \text { out }}$, and that $q\left(P_{\lambda}^{\prime}\right)=q\left(P^{\prime}\right)$ by $\mathscr{L}_{\lambda}$ "“ $=" \mathscr{L}$ after the previous identification.

(9.7) Proposition-Definition. In each of the following five cases (a)-(e), there exists a deformation $X_{\lambda} \supset C_{\lambda}$ as described above such that $\ell\left(P_{\lambda}\right)$ and $q\left(P_{\lambda}\right)$ satisfy the conditions ( $c f$. (9.6.3)).

(a) $\ell(P)=R\left(a_{2}\right)>1, q(P)=1 \Rightarrow \ell\left(P_{\lambda}\right)=1, q\left(P_{\lambda}\right)=R\left(a_{2}\right)>1$,

(b) $\ell(P)=R\left(a_{2}\right)>1, q(P)=0 \Rightarrow \ell\left(P_{\lambda}\right)=0, q\left(P_{\lambda}\right)=R\left(a_{2}\right)>1$,

(c) $\ell(P)=1, q(P)=R\left(a_{2}\right)>1 \Rightarrow \ell\left(P_{\lambda}\right)=0, q\left(P_{\lambda}\right)=R\left(a_{2}\right)>1$, 
(d) $\ell(P)=1, q(P)=0 \Rightarrow \ell\left(P_{\lambda}\right)=0, q\left(P_{\lambda}\right)=1$, and if $a_{2}=m-1$, then

(e) $\ell(P)=q(P)=1 \Rightarrow \ell\left(P_{\lambda}\right)=q\left(P_{\lambda}\right)=1$ and $P_{\lambda}$ has axial multiplicity 2 .

We call all these processes ( or $X_{\lambda}^{\circ} \supset C_{\lambda}$ ) the $L G$-deformations of $X \supset C$ at $P$.

(9.7.1) Remark. We put a table of possible values of $\ell(P), q(P)$ with the changes (a)-(e) described (cf. (9.5.1) and (9.6.3)).

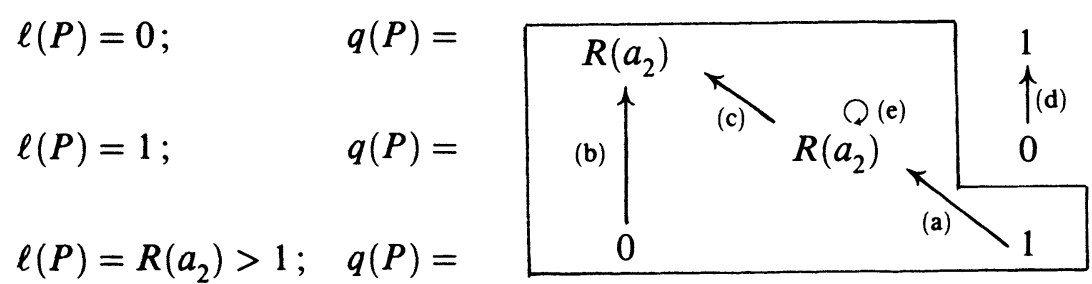

The enclosed cases correspond to $\ell(P)+q(P)>1$, and will be disproved in (9.8).

Proof. (a) (resp. (b)) We can choose (y) in (9.6) such that

$$
\phi \equiv y_{1}^{\ell(P)} y_{2}\left(y_{2}, y_{3}, y_{4}\right)^{2}
$$

and $y_{1} y_{3}$ (resp. $\left.y_{4}\right)$ generates $\mathscr{L}$ at $P$. Then we set $u=\left(y_{1} y_{3}, y_{4}\right)$ (resp. $\left.\left(y_{4}, y_{1} y_{3}\right)\right)$ and choose a general invariant $\psi \equiv y_{1} y_{3}\left(\right.$ resp. $\left.y_{4}\right) \bmod \left(y_{2}, y_{3}, y_{4}\right)^{2}$ such that g.c.d $\{\phi, \psi\}=1$ and $\operatorname{Sing}\{\phi=\psi=0\} \subset y_{1}$-axis. Thus (9.6.1) is satisfied. Since $y_{1} y_{3}$ (resp. $\left.y_{4}\right)$ appears in $\phi_{\lambda},\left(1 \mathrm{~b} .5\left(\mathrm{ii}^{\prime \prime}\right)\right)\left(\right.$ resp. $\left.\left(1 \mathrm{~b} .5\left(\mathrm{ii}^{\prime}\right)\right)\right)$ is satisfied and (9.6.2) is satisfied. Let $0<\lambda \ll 1$. Since $\phi_{\lambda}=0$ on $X_{\lambda}$, we see

$$
-y_{1}^{\ell(P)} y_{2} / \lambda \equiv y_{1} y_{3} \quad\left(\text { resp. } y_{4}\right) \quad \bmod I_{\lambda}^{2} \text {. }
$$

Thus $y_{3}+y_{1}^{\ell(P)-1} y_{2} / \lambda \in I_{\lambda}^{(2)}$ (resp. $y_{4}+y_{1}^{\ell(P)} y_{2} / \lambda \in I_{\lambda}{ }^{2}$ ), and $\ell\left(P_{\lambda}\right)=1$ (resp. 0) (cf. $(2.16(\mathrm{i}))$ ). Hence $\left\{y_{2}, y_{3}\right\}$ (resp. $\left\{y_{2}, y_{3}\right\}$ ) is an $\ell$-free $\ell$-basis of $\operatorname{gr}^{1}\left(\mathscr{O}, I_{\lambda}\right)$ at $P_{\lambda}$ (cf. (9.5.1)). Using (9.6.4), we see that $y_{2}$ is an $\ell$-free $\ell$-basis of $\mathscr{L}_{\lambda}$ at $P_{\lambda}$ by (9.7.2), whence $q\left(P_{\lambda}\right)=R\left(a_{2}\right)$.

(c) (resp. (d), (e)). Let $b=R\left(a_{2}\right)$. We can choose $(y)$ in (9.6) such that $\phi \equiv$ $y_{1} y_{3}\left(y_{2}, y_{3}, y_{4}\right)^{2}$ and $y_{1}{ }^{b} y_{2}$ (resp. $\left.y_{4}, y_{1}{ }^{b} y_{2}\right)$ generates $\mathscr{L}$ at $P$. Then we set $u=\left(y_{1}^{b} y_{2}, y_{4}\right)$ (resp. $\left.\left(y_{4}, y_{1}^{b} y_{2}\right),\left(y_{1}^{b} y_{2}, y_{4}\right)\right)$ and choose a general invariant $\psi \equiv y_{4}\left(\right.$ resp. $\left.y_{4}, y_{4}{ }^{2}\right) \bmod \left(y_{2}, y_{3}, y_{4}\right)^{3}$ such that g.c.d. $\{\phi, \psi\}=1$ and $\operatorname{Sing}\{\phi=\psi=0\} \subset y_{1}$-axis. Thus (9.6.1) is satisfied. Since $y_{1} y_{3}$ appears in $\phi,\left(1 \mathrm{~b} .5\left(\mathrm{ii}^{\prime \prime}\right)\right)$ is satisfied and $(9.6 .2)$ is satisfied. Let $0<\lambda \ll 1$. As in the cases (a) and (b), we also see

$$
-y_{1} y_{3} / \lambda \equiv y_{4} \quad\left(\text { resp. } y_{4}, 0\right) \quad \bmod I^{\lambda 2} .
$$


It is thus similarly seen that $\ell\left(P_{\lambda}\right)=0$ (resp. 0,1$),\left\{y_{2}, y_{3}\right\}$ (resp. $\left\{y_{2}, y_{3}\right\}$, $\left.\left\{y_{2}, y_{4}\right\}\right)$ is an $\ell$-free $\ell$-basis of $\operatorname{gr}_{C}^{1} \mathscr{O}$ at $P$, and $q\left(P_{\lambda}\right)=R\left(a_{2}\right)$ (resp. $\left.1, R\left(a_{2}\right)\right)$. In case $(\mathrm{e})$, we note that $\phi_{\lambda}$ restricted to the fixed axis $\left(y_{4}\right.$-axis) has a double zero, whence $P_{\lambda}$ has axial multiplicity 2 (1a.5).

(9.8) Theorem. We have $\ell(P)=q(P)=1$ and $\ell\left(P^{\prime}\right)+q\left(P^{\prime}\right)=1$.

Proof. By symmetry, it is enough to disprove $\ell(P)+q(P)>1$. Since it is enough to disprove a nearby extremal nbd of $X \supset C$, we will replace $X \supset C$ with its $L G$-deformation $(9.7)$ at $P$ or $P^{\prime}$ for several times. We treat three cases.

(9.8.1) Case $\ell(P)=0$ and $q(P)>1$. By replacing $X \supset C$ with its $L G$-deformation at $P^{\prime}(9.7 .1)$ for several times, we get a locally primitive $X \supset C$ with exactly two points $P$ and $P^{\prime}$ with indices $m, m^{\prime}(\geq 3)$ (9.6.4) such that $\ell\left(P^{\prime}\right)=0$. Since $L G$-deformation at $P^{\prime}$ does not change "nbd" of $P(9.6 .4)$, we have $\ell(P)=0$ and $q(P)>1$. By definition of $\ell(P)$ and $\ell\left(P^{\prime}\right)$, $P$ and $P^{\prime}$ are ordinary. Hence this contradicts (9.3) and we are done.

(9.8.2) Case $R\left(a_{2}\right)>1$. By replacing $X \supset C$ with its $L G$-deformation at $P$ (9.7.1) for several times, we get $X \supset C$ with $\ell(P)=0$ and $q(P)>1$ as in (9.8.1), whence we are done by (9.8.1).

(9.8.3) Case $R\left(a_{2}\right)=1$. Similarly to the previous case, we may assume that $\ell(P)=q(P)=1, P$ has axial multiplicity 2 , and that $P^{\prime}$ is ordinary, i.e. $\ell\left(P^{\prime}\right)=0$. We have $q\left(P^{\prime}\right)=1$ by $(9.8 .1)$.

(9.8.3.1) Since $\operatorname{gr}_{C}^{1} \mathscr{O}=\mathscr{L} \oplus \mathscr{O}(-1)$ (9.5), we have an $\ell$-splitting $\operatorname{gr}_{C}^{1} \mathscr{O}=$ $\mathscr{L} \widetilde{\oplus} \mathscr{M}$ for some submodule $\mathscr{M} \simeq \mathscr{O}(-1)$ (9.1.7(iii)), which is hence an $\ell$ invertible

$\mathscr{O}_{C}$-module (9.1.1). We have $q l_{C}(\mathscr{L})=P^{\sharp}+P^{b}$ and $q l_{C}(\mathscr{M})=-1+\left(m^{\prime}-a_{2}^{\prime}\right) P^{b}$ (9.5.1) because $q l \operatorname{deg}(\mathscr{M}, P)=R\left(a_{4}\right)=0$ by $\ell(P)=q(P)=R\left(a_{2}\right)=R\left(a_{3}\right)=$ 1 and $q l \operatorname{deg}\left(\mathscr{M}, P^{\prime}\right)=R^{\prime}\left(a_{2}^{\prime}\right)$ by $\ell\left(P^{\prime}\right)=0=R^{\prime}\left(a_{4}^{\prime}\right)$ and $q\left(P^{\prime}\right)=1=R^{\prime}\left(a_{3}^{\prime}\right)$.

(9.8.3.2) Let $y=\left(y_{1}, \ldots, y_{4}\right)$ be an $\ell$-coordinate system and an $\ell$-equation $\phi$ at $P$ such that $C^{\sharp}=y_{1}$-axis, $\phi \equiv y_{1} y_{3}\left(y_{2}, y_{3}, y_{4}\right)^{2}(2.16)$ and $\mathscr{L}$ is generated by $y_{1} y_{2}$ at $P(9.1 .1)$ (or cf. (9.4.5)). Since $P$ has axial multiplicity 2 , we see $\phi \equiv y_{1} y_{3}+g y_{4}{ }^{2} \bmod \left(y_{2}, y_{3}\right)^{2}+\left(y_{2}, y_{3}\right) y_{4}+y_{4}{ }^{3}$, where $g$ is an invariant unit $\in \mathbb{C}\{y\}$. Thus by replacing $y_{4}$ by $g^{ \pm 1 / 2} y_{4}$, we may further assume

$$
\phi \equiv y_{1} y_{3}+y_{4}^{2} \bmod \left(y_{2}, y_{3}\right)^{2}+\left(y_{2}, y_{3}\right) y_{4}+\left(y_{4}{ }^{3}\right) \text {. }
$$

(9.8.3.3) Let $J$ be a $C$-laminal ideal of width 2 such that $J / I^{(2)}=\mathscr{L}$. Then an argument very similar to (9.3.2) or (9.4.6) shows that $J$ is a nested c.i. outside $\left\{P, P^{\prime}\right\}$, that we have equalities $\operatorname{Im} \alpha_{J}=\operatorname{Ker} \beta_{J}=\mathscr{L}$ with $\ell$-structure and an $\ell$-isomorphism $\operatorname{gr}^{1}(\mathscr{O}, J) \simeq \mathscr{M}$, and that $J$ is $(1,2)$-monomializable 
at ordinary point $P^{\prime}$. We see by $(9.8 .3 .2)$ that $\left(y_{4}, y_{2}, y_{3}\right)$ is a $(1,2,2)$ monomializing $\ell$-basis of the second kind for $I \supset J$ at $P$. Let $\mathscr{D}$ be the $\ell$-invertible $\mathscr{O}_{C}$-module defined in (8.11.1(iii)) from $\left(y_{4}, y_{2}, y_{3}\right)$, then we have a normalized expression $q l_{C} \mathscr{D}=P^{\sharp}$. Hence we have an inequality

$$
-l \operatorname{deg}_{C} \mathscr{M} \leq \frac{1}{2} l \operatorname{deg}_{C} \mathscr{L}+\frac{1}{2} l \operatorname{deg}_{C} \mathscr{D}
$$

by (8.12(ii)) (with $d=2, t=2, s=1, s^{\prime}=0$ ). This reduces to

$$
\frac{a_{2}^{\prime}}{m^{\prime}} \leq \frac{1}{2}\left(\frac{1}{m}+\frac{1}{m^{\prime}}\right)+\frac{1}{2 \cdot m}=\frac{1}{m}+\frac{1}{2 m^{\prime}} \leq \frac{1}{2}
$$

by $m, m^{\prime} \geq 3$, where we used $l \operatorname{deg}_{C} \mathscr{M}=-a_{2}^{\prime} / m^{\prime}, l \operatorname{deg}_{C} \mathscr{L}=1 / m+1 / m^{\prime}$ (9.8.3.1), and $l \operatorname{deg}_{C} \mathscr{D}=1 / m$. Thus $2 a_{2}^{\prime} \leq m^{\prime}$ and we see $2 a_{2}^{\prime}<m^{\prime}$ by $m^{\prime} \geq 3$ and $\left(a_{2}^{\prime}, m^{\prime}\right)=1$. Also by (8.12), we have $\ell$-isomorphisms

$$
\begin{aligned}
& \operatorname{gr}^{2,0}(\mathscr{O}, J) \simeq \mathscr{L} \quad(d=2, n=2, q=1, r=0), \\
& \operatorname{gr}^{2,1}(\mathscr{O}, J) \simeq \mathscr{M}^{\widetilde{\otimes} 2} \widetilde{\otimes} \mathscr{D} .
\end{aligned}
$$

and an $\ell$-exact sequence

$$
0 \rightarrow \mathscr{M}^{\widetilde{\otimes} 2} \widetilde{\otimes} \mathscr{D} \rightarrow \mathrm{gr}_{C}^{0} J \rightarrow \mathscr{L} \rightarrow 0 .
$$

We note that $q l_{C}\left(\mathscr{M}^{\widetilde{\otimes} 2} \widetilde{\otimes} \mathscr{D}\right)=2 q l_{C}(\mathscr{M})+q l_{C}(\mathscr{D})=-1+P^{\sharp}+\left(m^{\prime}-2 a_{2}^{\prime}\right) P^{b}$ is a normalized expression, and that the sequence is $\ell$-split as

$$
\operatorname{gr}_{C}^{0} J=\left(\mathscr{M}^{\widetilde{\otimes} 2} \widetilde{\otimes} \mathscr{D}\right) \widetilde{\oplus} \mathscr{L}_{J}
$$

for some $\mathscr{L}_{J} \subset \operatorname{gr}_{C}^{0} J \quad \ell$-isomorphic to $\mathscr{L}\left(9.1 .8(\right.$ ii) $)$ by $q l_{C}(\mathscr{L})=P^{\sharp}+P^{b}$ and $m^{\prime}>2 a_{2}^{\prime}$.

(9.8.3.4) Let $K$ be a $C$-primary ideal such that $J \supset K \supset F^{1} J$ and $K / F^{1} J=$ $\mathscr{L}_{J}$. Then $K$ is a $C$-laminal ideal of width 3 which is a nested c.i. outside $\left\{P, P^{\prime}\right\}$, and we have $\ell$-isomorphisms $\operatorname{Ker} \beta_{K}=\operatorname{Ker} \beta_{J}=\mathscr{L}$ and $\operatorname{gr}^{1}(\mathscr{O}, K)=$ $\operatorname{gr}^{1}(\mathscr{O}, J) \simeq \mathscr{M}$ by $(8.14)$. Then $K$ is $(1,3)$-monomializable at $P^{\prime}(8.15 .1)$ and the $(1,2,2)$-monomializing $\ell$-basis $\left(y_{4}, y_{2}, y_{3}\right)$ for $I \supset J$ at $P$ lifts to a $(1,3,2)$-monomializing $\ell$-basis (denoted by the same) $\left(y_{4}, y_{2}, y_{3}\right)$ (by abuse of language) of the second kind for $I \supset K$ at $P$ with the same attached semiinvariant by (8.16). Thus the invertible sheaf $\mathscr{D}$ for $K$ is the same as the one in (9.8.3.3). Then we have an inequality by $(8.12$ (ii))

$$
-l \operatorname{deg}_{C} \mathscr{M} \leq \frac{1}{3} l \operatorname{deg}_{C} \mathscr{L}+\frac{1}{2} l \operatorname{deg}_{C} \mathscr{D},
$$

which reduces to

$$
\frac{1}{m}<\frac{a_{2}^{\prime}}{m^{\prime}} \leq \frac{1}{3}\left(\frac{1}{m}+\frac{1}{m^{\prime}}\right)+\frac{1}{2 \cdot m}=\frac{5}{6 \cdot m}+\frac{1}{3 m^{\prime}},
$$

where the first inequality follows from $w_{P}(0)=1 / m<1-w_{P^{\prime}}(0)=a_{2}^{\prime} / m^{\prime}$ (2.3.3) and (4.9(i)). Then we treat two cases. 
(9.8.3.6) Case $m=3$. By (9.8.3.5), we have $1 / 3<5 / 18+1 / 3 m^{\prime}$, whence $m^{\prime}=3,4,5$. If $m^{\prime}=3$, then $a_{2}^{\prime}=2$ by (9.8.3.5), which contradicts $2 a_{2}^{\prime}<m^{\prime}$ proved in (9.8.3.3). We see that $(9.8 .3 .5)$ reduces to $4 / 3<a_{2}^{\prime} \leq 5 \cdot 4 /(6 \cdot 3)+$ $1 / 3=13 / 9$ if $m^{\prime}=4$, and to $5 / 3<a_{2}^{\prime} \leq 5 \cdot 5 /(6 \cdot 3)+1 / 3=31 / 18$ if $m^{\prime}=5$. These are both impossible, and the case $m=3$ cannot occur.

(9.8.3.7) Case $m \geq 4$. Since

$$
\frac{5}{6 \cdot m}+\frac{1}{3 m^{\prime}} \leq \frac{5}{6 \cdot 4}+\frac{1}{3 \cdot 3}=\frac{23}{72}<\frac{1}{3},
$$

we see that $3 a_{2}^{\prime}<m^{\prime}$ by (9.8.3.5). Then we continue as in (9.8.3.3). By (8.12(ii)), we have $\ell$-isomorphisms

$$
\begin{aligned}
& \operatorname{gr}^{3,0}(\mathscr{O}, K) \simeq \mathscr{L} \quad(d=3, n=3, q=1, r=0), \\
& \operatorname{gr}^{3,1}(\mathscr{O}, K) \simeq \mathscr{M}^{\widetilde{\otimes} 3} \widetilde{\otimes} \mathscr{D} .
\end{aligned}
$$

and an $\ell$-exact sequence

$$
0 \rightarrow \mathscr{M}^{\widetilde{\otimes} 3} \widetilde{\otimes} \mathscr{D} \rightarrow \mathrm{gr}_{C}^{0} K \rightarrow \mathscr{L} \rightarrow 0 .
$$

Since $q l_{C}\left(\mathscr{M}^{\widetilde{\otimes} 3} \widetilde{\otimes} \mathscr{D}\right)=3 q l_{C}(\mathscr{M})+q l_{C}(\mathscr{D})=-1+P^{\sharp}+\left(m^{\prime}-3 a_{2}^{\prime}\right) P^{\mathrm{b}}$ is a normalized expression, the sequence is also $\ell$-split as $\operatorname{gr}_{C}^{0} K=\mathscr{M}^{\widetilde{\otimes} 3} \widetilde{\otimes} \mathscr{D} \widetilde{\oplus} \mathscr{L}_{K}$ for some submodule $\mathscr{L}_{K} \ell$-isomorphic to $\mathscr{L}$ (9.1.8). Then as in (9.8.3.4), we can define a $C$-laminal ideal $H$ of width 4 such that $K \supset H \supset F^{1} K$ and $H / F^{1} K=\mathscr{L}_{K}$. We see that $H$ is a nested c.i. outside $\left\{P, P^{\prime}\right\} ; H$ is $(1,4)$-monomializable at $P^{\prime}(8.15) ;\left(y_{4}, y_{2}, y_{3}\right)$ lifts to a $(1,4,2)$-monomializing $\ell$-basis of the second kind for $I \supset H$ at $P$ with the same attached semi-invariant; we have $\ell$-isomorphisms $\operatorname{gr}^{1}(\mathscr{O}, H) \simeq \mathscr{M}$ and $\operatorname{Ker} \beta_{H} \simeq \mathscr{L}$ and the same $\mathscr{D}$. Thus we have

$$
\frac{1}{m}<\frac{a_{2}^{\prime}}{m^{\prime}} \leq \frac{1}{4} l \operatorname{deg}_{C} \mathscr{L}+\frac{1}{2} l \operatorname{deg}_{C} \mathscr{D}=\frac{1}{4}\left(\frac{1}{m}+\frac{1}{m^{\prime}}\right)+\frac{1}{2 \cdot m}=\frac{3}{4 \cdot m}+\frac{1}{4 m^{\prime}}
$$

just as (9.8.3.5). Then by $1 / m<3 /(4 m)+1 /\left(4 m^{\prime}\right)$, we see $1 / m<1 / m^{\prime}$, whence $a_{2}^{\prime} / m^{\prime} \leq 3 /(4 m)+1 /\left(4 m^{\prime}\right)<1 / m^{\prime}$. Thus $a_{2}^{\prime}=0$, which is a contradiction. Thus we must have $\ell(P)+q(P)=1$ and $(9.8)$ is proved.

(9.9) Let the notation and assumptions be as in (9.1). Then $\operatorname{gr}_{C}^{1} \mathscr{O}=\mathscr{L} \oplus$ $\mathscr{O}(-1)$, and by (9.1.1) there is a submodule $\mathscr{M} \simeq \mathscr{O}(-1)$ such that $\operatorname{gr}_{C}^{1} \mathscr{O}=$ $\mathscr{L} \widetilde{\oplus} \mathscr{M}$. Then by $(9.8), q l \operatorname{deg}(\mathscr{M}, P)=R\left(a_{2}\right)$ and $q l \operatorname{deg}\left(\mathscr{M}, P^{\prime}\right)=R^{\prime}\left(a_{2}^{\prime}\right)$ (9.5.1). In particular $q l_{C}(\mathscr{M})=-1+\left(m-a_{2}\right) P^{\sharp}+\left(m^{\prime}-a_{2}^{\prime}\right) P^{b}$. Thus by $(2.10)$ and $\operatorname{gr}_{C}^{0} \omega \simeq \mathscr{O}(-1)$, we have

(9.9.1) Proposition. There is an $\ell$-isomorphism $\mathscr{M} \simeq \operatorname{gr}_{C}^{0} \omega$.

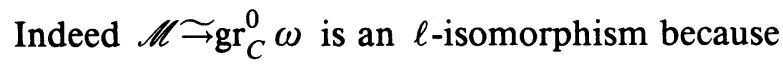

$$
q l \operatorname{deg}(\mathscr{M}, Q)=q l \operatorname{deg}\left(\operatorname{gr}_{C}^{0} \omega, Q\right) \text { for } Q=P, P^{\prime}
$$

(cf. comment after ( $\widetilde{\oplus} .2)$ in (8.8.4)). 
(9.9.2) We note that $\omega_{X}^{*}$ has a structure of an $\ell$-invertible $\mathscr{O}_{X}$-module $\omega_{X}{ }^{\widetilde{\otimes}(-1)}$. Hence $\operatorname{gr}_{C}^{0}\left(\omega_{X}^{*}\right)$ is $\ell$-isomorphic to

$$
\mathscr{O}_{C} \tilde{\otimes} \omega_{X}^{\widetilde{\otimes}(-1)} \simeq\left(\mathscr{O}_{C} \widetilde{\otimes} \omega_{X}\right)^{\widetilde{\otimes}(-1)} \simeq\left(\mathrm{gr}_{C}^{0} \omega\right)^{\widetilde{\otimes}(-1)}
$$

by (8.8.6(i)) and $(\tilde{\otimes} \tilde{\otimes} .3)$ in (8.8.4), whence we have a normalized expression $q l_{C}\left(\mathrm{gr}_{C}^{0} \omega_{X}^{*}\right)=-q l_{C}\left(\mathrm{gr}_{C}^{0} \omega\right)=-q l_{C}(\mathscr{M})=-1+a_{2} P^{\sharp}+a_{2}^{\prime} P^{b}$. We also have an $\ell$-isomorphism

$$
\operatorname{gr}_{C}^{1}\left(\omega_{X}^{*}\right) \simeq\left(\operatorname{gr}_{C}^{1} \mathscr{O}\right) \widetilde{\otimes} \omega_{X}^{*} \simeq \mathscr{L} \widetilde{\otimes} \mathscr{M}^{\widetilde{\otimes}(-1)} \widetilde{\oplus} \mathscr{O}_{C},
$$

by $(8.8 .6(i))$ and (8.8.4). Then

(9.9.3) Lemma. We have an isomorphism

$$
H^{0}\left(\omega_{X}^{*} / F^{2} \omega_{X}^{*}\right) \simeq H^{0}\left(F^{1} \omega_{X}^{*} / F^{2} \omega_{X}^{*}\right) \simeq H^{0}\left(\mathscr{L} \widetilde{\otimes} \mathscr{M}^{\widetilde{\otimes}(-1)}\right) \oplus H^{0}\left(\mathscr{O}_{C}\right),
$$

and let

$$
h: H^{0}\left(\omega_{X}^{*}\right) \rightarrow H^{0}\left(\omega_{X}^{*} / F^{2} \omega_{X}^{*}\right) \rightarrow H^{0}\left(\mathscr{O}_{C}\right) \simeq \mathbb{C}
$$

be the projection to the factor $H^{0}\left(\mathscr{O}_{C}\right)$. Then every global section $s \in H^{0}\left(\omega_{X}^{*}\right)$ such that $h(s) \neq 0$ defines a zero locus $(s)_{0}$ which is a normal surface with only rational double points. (To be precise, $(s)_{0}$ is smooth outside $\left\{P, P^{\prime}\right\}$, has $A_{m w-1}$-type (resp. $A_{m^{\prime} w^{\prime}-1^{-t y p e}}$ ) singularity at $P\left(\right.$ resp. $\left.P^{\prime}\right)$, where $w$ (resp. $\left.w^{\prime}\right)$ is the axial multiplicity of $(X, P) \quad\left(\right.$ resp. $\left.\left.\left(X, P^{\prime}\right)\right)\right)$.

Proof. The first assertion follows from $H^{0}\left(\mathrm{gr}_{C}^{0}\left(\omega_{X}^{*}\right)\right) \simeq H^{0}\left(\mathscr{O}_{C}(-1)\right)=0$ (9.9.2). Let $s \in H^{0}\left(\omega_{X}^{*}\right)$ be such that $h(s) \neq 0$. Then $s \in H^{0}\left(F^{1} \omega_{X}^{*}\right)$ by $H^{0}\left(\operatorname{gr}_{C}^{0} \omega_{X}^{*}\right)=0$.

If $Q \neq P, P^{\prime}$, then the image of $s(Q)$ in $\left(\mathrm{gr}_{C}^{1} \omega_{X}^{*}\right) \otimes \mathbb{C}(Q)=\omega_{X}^{*} \otimes I_{C} / I_{C}{ }^{2} \otimes$ $\mathbb{C}(Q)$ is nonzero because $h(s)$ generates the subspace $\mathscr{O}_{C} \otimes \mathbb{C}(Q) \simeq \mathbb{C}$. Hence $(s)_{0}$ is smooth at $Q$.

By symmetry we need to consider $(s)_{0}$ only at $P^{\sharp}$. By $(9.5 .1)$ and $\ell(P)=$ 0,1 , we can take an $\ell$-coordinate system $y_{1}, \ldots, y_{4}$ such that $C^{\sharp}=y_{1}$-axis, wt $y_{i} \equiv a_{i}(m) \quad(i \in[1,4]), y_{2}$ is an $\ell$-free $\ell$-basis of $\mathscr{M}$ at $P, y_{3}$ (resp. $\left.y_{4}\right)$ is an $\ell$-free $\ell$-basis of $\mathscr{L}$ at $P$, and $\phi \equiv y_{4}\left(\right.$ resp. $\left.y_{1} y_{3}\right) \bmod \left(y_{2}, y_{3}, y_{4}\right)^{2}$ if $\ell(P)=0$ (resp. 1). Let $\theta$ be an $\ell$-free $\ell$-basis of $\omega_{X}^{*}$ at $P$. By the assumption $h(s) \neq 0, \mathscr{O}_{C^{\sharp}}$-component of $s_{P}$ in the decomposition at $P^{\sharp}$

$$
\operatorname{gr}^{1}\left(\omega_{X}^{*}\right)^{\sharp}=\left(\mathscr{L} \widetilde{\otimes} \mathscr{M}^{\otimes(-1)} \widetilde{\otimes} \operatorname{gr}_{C}^{0} \omega\right)^{\sharp} \oplus \mathscr{O}_{C^{\sharp}}=\mathscr{O}_{C^{\sharp}}\left(y_{3}, y_{4}\right) \theta \oplus \mathscr{O}_{C^{\sharp}} y_{2} \theta
$$

generates $\mathscr{O}_{C^{\sharp}} y_{2} \theta$. Then $s=f \theta$ for some semi-invariant $f \in\left(y_{2}, y_{3}, y_{4}\right) \mathbf{C}\{y\}$ such that wt $f \equiv a_{2} \equiv$ wt $y_{2}(m)$ and $\partial f / \partial y_{2}(0) \neq 0$. Thus there exist an invariant unit $u \in \mathbb{C}\{y\}$ and a semi-invariant $g \in\left(y_{3}, y_{4}\right) \mathbb{C}\left\{y_{1}, y_{3}, y_{4}\right\}$ such that wt $g \equiv a_{2}(m)$ and $f=u \cdot\left(y_{2}-g\right)$ (Weierstrass Preparation Theorem). 
The zero locus $(s)_{0}^{\sharp}$ of $s$ in the canonical cover $\left(X^{\sharp}, P^{\sharp}\right)$ is thus isomorphic to a hypersurface in $\left(y_{1} y_{3} y_{4}\right.$-space, 0$)$ with defining equation $\psi\left(y_{1}, y_{3}, y_{4}\right)=$ $\phi\left(y_{1}, g, y_{3}, y_{4}\right) \in \mathbb{C}\left\{y_{1}, y_{3}, y_{4}\right\}$. By the congruence relation on $\phi$, we have $\psi \equiv y_{4}\left(\right.$ resp. $\left.y_{1} y_{3}\right) \bmod \left(y_{3}, y_{4}\right)^{2}$.

We will see that $(s)_{0}^{\sharp}$ is a $A_{w-1}$-type singularity. Indeed if $\psi \equiv y_{4}$ then the axial multiplicity $w$ is 1 and $(s)_{0}^{\sharp}$ is smooth. We consider the case $\psi \equiv y_{1} y_{3}$. Since $\partial \psi / \partial y_{1} \equiv y_{3} \bmod \left(y_{3}, y_{4}\right)^{2}$ has $w t \equiv a_{3} \not \equiv 0(m)$ and $\partial \psi / \partial y_{3} \equiv y_{1} \bmod \left(y_{3}, y_{4}\right)$ has $\mathrm{wt} \equiv a_{1} \not \equiv 0(m)$, we see $\partial \psi / \partial y_{1} \equiv y_{3} \bmod$ $\left(y_{3}{ }^{2}, y_{3} y_{4}, y_{1} y_{4}{ }^{2}\right)$ and $\partial \psi / \partial y_{3} \equiv y_{1} \bmod \left(y_{3}, y_{1} y_{4}\right)$ because wt $y_{4} \equiv 0(m)$. Hence $\partial \psi / \partial y_{1}$ and $\partial \psi / \partial y_{3}$ generate the ideal $\left(y_{1}, y_{3}\right)$ in a nbd of origin by Nakayama's lemma, and they define $y_{4}$-axis. On the other hand, by wt $g \equiv a_{2}$ $(m)$, we see $g\left(0,0, y_{4}\right)=0$ and $\psi\left(0,0, y_{4}\right)=\phi\left(0,0,0, y_{4}\right)=c y_{4}{ }^{w}+\cdots$, where $w$ is the axial multiplicity of $(X, P)(1 \mathrm{a} .5)$. Thus $(s)_{0}^{\sharp}$ is an isolated singularity, and it is easy to see $(s)_{0}^{\sharp}$ is $\boldsymbol{\mu}_{m}$-isomorphic to a $A_{w-1}$-singularity $y_{1} y_{3}=y_{4}{ }^{w}$ (by analytic approximation).

Hence $\left((s)_{0}, P\right)=(s)_{0}^{\sharp} / \mu_{m}$ is isomorphic to a $A_{m w-1}$-type singularity: $z_{1} z_{3}=z_{4}^{m w}$, where $z_{1}=y_{1}{ }^{m}, z_{3}=y_{3}{ }^{m}, z_{4}=y_{4}$.

(9.9.4) Theorem. The homomorphism

$$
h: H^{0}\left(\omega_{X}^{*}\right) \rightarrow H^{0}\left(\mathscr{O}_{C}\right)=\mathbb{C}
$$

defined in (9.9.3) is a surjection.

We will prove the theorem in several steps.

(9.9.5) Let $J_{2}$ be the $C$-laminal ideal of width 2 such that $J_{2} / I^{(2)}=\mathscr{M}$. Since $J_{2}, I^{(2)}, \mathscr{M}$ are saturated subquotients of $\mathscr{O}_{X}$, the surjection $J_{2} \rightarrow \mathscr{M}$ induces a surjection of canonical liftings at $P$ (and also at $P^{\prime}$ ) by (8.8.1). Thus the homomorphism $\alpha_{J}$ for $J=J_{2}(8.2 .2)$ is an $\ell$-surjection

$$
\alpha_{2}: \operatorname{gr}_{C}^{0} J_{2} \rightarrow \mathscr{M} \text {. }
$$

We will inductively define $C$-laminal ideals $J_{n}$ of width $n$.

(9.9.6) Lemma-Definition. Let $J_{n}$ be a C-laminal ideal of width $n(\geq 2)$ such that the homomorphism $\alpha_{J}$ for $J=J_{n}$ (8.2.2) is an $\ell$-surjection

$$
\alpha_{n}: \operatorname{gr}_{C}^{0} J_{n} \rightarrow \mathscr{M} \text {. }
$$

Then the $\ell$-exact sequence $(8.8 .2(\mathrm{v}))$

$$
0 \rightarrow \operatorname{Ker} \alpha_{n} \rightarrow \operatorname{gr}_{C}^{0} J_{n} \rightarrow \mathscr{M} \rightarrow 0
$$

is $\ell$-split, that is $\mathrm{gr}_{C}^{0} J_{n}=\left(\operatorname{Ker} \alpha_{n}\right) \widetilde{\oplus} \mathscr{M}_{n}$ for some submodule $\mathscr{M}_{n}$ of $\operatorname{gr}_{C}^{0} J_{n}$ such that the induced map $\mathscr{M}_{n} \rightarrow \mathscr{M}^{n}$ is an l-isomorphism. Let $J_{n+1}$ be the $C$ primary ideal such that $J_{n} \supset J_{n+1} \supset F^{1} J_{n}$ and $J_{n+1} / F^{1} J_{n}=\mathscr{M}_{n}$. Then the 
natural $\ell$-surjections $\operatorname{gr}_{C}^{0} J_{n+1} \rightarrow \mathscr{M}_{n}\left(\subset \operatorname{gr}_{C}^{0} J_{n}\right)\left(\right.$ cf. (8.8.1)) and $\mathscr{M}_{n} \stackrel{\sim}{\longrightarrow} \mathscr{M}$ induce an $\ell$-surjection

$$
\alpha_{n+1}: \mathrm{gr}_{C}^{0} J_{n+1} \rightarrow \mathscr{M} .
$$

In particular, $J_{n+1}$ is a $C$-laminal ideal, hence of width $n+1$.

Proof. We only need to prove $\ell$-splitness by induction on $n$. By inductive construction of $J_{n}$, we have $\mathscr{M}=\operatorname{Ker} \beta_{n}$ and $\mathscr{L}=\operatorname{gr}^{1}\left(\mathscr{O}, J_{n}\right)$ (8.2), where $\beta_{n}=\beta_{J}$ for $J=J_{n}$ (8.2.2). Hence by $E\left(J_{n}\right)$ (8.2.2) and (8.4), we have an injection $\mathscr{O}_{C} \simeq \mathscr{L}^{\otimes d} \rightarrow \operatorname{Ker} \alpha_{n}$. Thus $\operatorname{deg}_{C} \operatorname{Ker} \alpha_{n}>\operatorname{deg} \mathscr{M}$ and the sequence is $\ell$-split $(9.1 .8(\mathrm{ii}))$.

(9.9.6.1) Let us fix a number $e \in \mathbb{N}$ which is divisible by axial multiplicities of $(X, P)$ and $\left(X, P^{\prime}\right)$. Let $C(n) \subset X$ be the closed subscheme defined by $F^{n e}\left(\mathscr{O} ; J_{e}\right)$. Then we have

(9.9.7) Key Lemma. For an arbitrary $n \geq 2, F^{n e}\left(\mathscr{O}, J_{e}\right)$ is equal to the symbolic nth power $J_{e}^{(n)}, J_{n e} / F^{n e}\left(\mathscr{O}, J_{e}\right)$ is an $\ell$-invertible $\mathscr{O}_{C(n-1)}$-module, and we have an $\ell$-isomorphism

$$
\theta(n): F^{n e}\left(\omega_{X}^{*}, J_{n e}\right) / F^{n e}\left(\omega_{X}^{*}, J_{e}\right) \stackrel{\sim}{\longrightarrow} \omega_{X}^{*} \widetilde{\otimes}\left(J_{n e} / F^{n e}\left(\mathscr{O}, J_{e}\right)\right) \stackrel{\sim}{\longrightarrow} \mathscr{O}_{C(n-1)}
$$

and inclusion $F^{n e m m^{\prime}}\left(\omega_{X}^{*}, J_{e}\right) \subset J_{e}^{n} \cdot \omega_{X}^{*}$.

Proof. Since $\omega_{X}^{*}$ is an $\ell$-invertible $\mathscr{O}_{X}$-module, we have natural $\ell$-isomorphisms by $(8.8 .6(\mathrm{i}))$

$$
\omega_{X}^{*} \tilde{\otimes} J_{n e} \simeq F^{n e}\left(\omega_{X}^{*}, J_{n e}\right) \quad \text { and } \omega_{X}^{*} \widetilde{\otimes} F^{n e}\left(\mathscr{O}, J_{e}\right) \simeq F^{n e}\left(\omega_{X}^{*}, J_{e}\right)
$$

inducing an $\ell$-isomorphism

$$
F^{n e}\left(\omega_{X}^{*}, J_{n e}\right) / F^{n e}\left(\omega_{X}^{*}, J_{e}\right) \simeq \omega_{X}^{*} \widetilde{\otimes}\left(J_{n e} / F^{n e}\left(\mathscr{O}, J_{e}\right)\right)
$$

by $(\tilde{\otimes} .5)$ in (8.8.4), provided that $J_{n e} \supset F^{n e}\left(\mathscr{O}, J_{e}\right)$ which is to be proved later.

(9.9.8) Let us first assume that $\mathscr{F}=J_{n e} / F^{n e}\left(\mathscr{O}, J_{e}\right)$ is an $\ell$-invertible $\mathscr{O}_{C(n-1)}$-module and $F^{n e}\left(\mathscr{O}, J_{e}\right)=J_{e}^{(n)}$. Then $F^{n e}\left(\mathscr{O}, J_{e}\right) \subset I^{(2)}$ and $\alpha_{n e}$ gives the identification $J_{n e}+I^{(2)} / I^{(2)}=\mathscr{M}$, whence the identity map induces the surjection $\mathscr{F} \rightarrow \mathscr{M}$ of saturated subquotients and it is an $\ell$-surjection (8.8.1). Thus by $(\widetilde{\otimes} .0)$ and $(\widetilde{\otimes} .4)$ in (8.8.4), we have an $\ell$-surjection

$$
\sigma: \omega_{X}^{*} \tilde{\otimes} \mathscr{F} \widetilde{\otimes} \mathscr{O}_{C} \rightarrow\left(\omega_{X}^{*} \tilde{\otimes} \mathscr{M} \simeq\right) \mathscr{O}_{C},
$$

where the last $\ell$-isomorphism is due to (9.9.1). Then it is an $\ell$-isomorphism (8.8.2(v)) by $\operatorname{Ker} \sigma=0$. Hence the $\ell$-invertible $\mathscr{O}_{C(n-1)}$-module $\omega_{X}^{*} \widetilde{\otimes} \mathscr{F}$ has an $\ell$-free $\ell$-basis with $\mathrm{wt} \equiv 0 \bmod m$ (resp. $m^{\prime}$ ), hence $\ell$-trivial at $P$ (resp. $\left.P^{\prime}\right)$. Then we see that $\omega_{X}^{*} \widetilde{\otimes} \mathscr{F}$ is an invertible $\mathscr{O}_{C(n-1)}$-module such that

$$
\left(\omega_{X}^{*} \tilde{\otimes} \mathscr{F}\right) \otimes \mathscr{O}_{C} \simeq\left(\left(\omega_{X}^{*} \tilde{\otimes} \mathscr{F}\right) \tilde{\otimes} \mathscr{O}_{C} \simeq\right) \mathscr{O}_{C}
$$


by $(\widetilde{\otimes} .8)$ in (8.8.4). Since $H^{1}\left(\mathscr{O}_{C(n-1)}\right)=0$, we have an isomorphism $\theta(n)$ : $\omega_{X}^{*} \widetilde{\otimes} \mathscr{F} \stackrel{\sim}{\longrightarrow} \mathscr{O}_{C(n-1)}$ of invertible $\mathscr{O}_{C(n-1)}$-modules. Since $\omega_{X}^{*} \widetilde{\otimes} \mathscr{F}$ and $\mathscr{O}_{C(n-1)}$ are $\ell$-trivial at $P$ and $P^{\prime}, \theta(n)$ is an $\ell$-isomorphism.

(9.9.9) It remains to show that $F^{n e}\left(\mathscr{O}, J_{e}\right)=J_{e}^{(n)}, J_{n e} / F^{n e}\left(\mathscr{O}, J_{e}\right)$ is an $\ell$-invertible $\mathscr{O}_{C(n-1)}$-module, and the last inclusion of (9.9.7) at each point $Q \in C$. Let $Q \in C-\left\{P, P^{\prime}\right\}$. By the surjectivity of $\alpha_{n e}$, let $\left\{u_{1}, u_{2}\right\}$ be a basis of $\operatorname{gr}_{C}^{1}=I / I^{2}$ at $P$ such that $\mathscr{O}_{C, Q} u_{1}=\mathscr{M}_{Q}$ and $u_{1} \in J_{n e}$. Then by (8.3(i)), we see that $J_{e}=\left(u_{1}, u_{2}{ }^{e}\right), J_{n e}=\left(u_{1}, u_{2}^{n e}\right)$, and $F^{n e}\left(\mathscr{O}, J_{e}\right)=J_{e}{ }^{n}$ near $Q$. In particular, we have the global equality $F^{n e}\left(\mathscr{O}, J_{e}\right)=J_{e}^{(n)}$ since both are $C$-primary. Furthermore, by $u_{2}^{n e} \in F^{n e}\left(\mathscr{O}, J_{e}\right)$, we see that $J_{n e} / F^{n e}\left(\mathscr{O}, J_{e}\right) \simeq$ $\mathscr{O} /\left\{\left(u_{1}, u_{2}^{e}\right)^{n}: u_{1}\right\} \simeq \mathscr{O} / J_{e}^{n-1}$. Thus the sheaf is invertible and the inclusion at $Q$ is obvious by $F^{n e}\left(\mathscr{O}, J_{e}\right)=J_{e}^{n}$.

(9.9.10) By symmetry, it is enough to consider at $P$. Let $y=\left(y_{1}, \ldots, y_{4}\right)$ and $\phi$ be an $\ell$-coordinate system and an $\ell$-equation at $P$ such that $C^{\sharp}=y_{1}$ axis, wt $y_{i} \equiv a_{i}(m) \quad(i \in[1,4]), \phi \equiv y_{4}\left(\right.$ resp. $\left.y_{1} y_{3}\right) \bmod \left(y_{2}, y_{3}, y_{4}\right)^{2}$ if $\ell(P)=0$ (resp. 1), $y_{2} \in J_{n e}^{\sharp}$, where $J_{n e}^{\sharp}$ is the canonical lifting of $J_{n e}$ at $P$. We note that we used $\ell$-surjection $\alpha_{n e}$ for the last condition. We treat two cases.

(9.9.11) Case $\ell(P)=0$. Again by $(8.3(\mathrm{i})),\left(y_{3}, y_{2}\right)$ is a $(1, e)$-monomializing (resp. $(1, n e)$-monomializing) $\ell$-basis for $I \supset J_{e}$ (resp. $I \supset J_{n e}$ ) at $P$. Thus as in (9.9.9), the canonical liftings are calculated as $J_{e}^{\sharp}=\left(y_{2}, y_{3}{ }^{e}\right), J_{n e}^{\sharp}=$ $\left(y_{2}, y_{3}{ }^{n e}\right)$, and $F^{n e}\left(\mathscr{O}, J_{e}\right)^{\sharp}=\left(J_{e}^{\sharp}\right)^{n}$. Hence $J_{n e}^{\sharp} / F^{n e}\left(\mathscr{O}, J_{e}\right)^{\sharp} \simeq \mathscr{O} / J_{3}^{\sharp n-1}$ is similarly checked. By $F^{n e}\left(\mathscr{O}, J_{e}\right)^{\sharp}=J_{e}^{\sharp n}$, the inclusion follows from the easy

(9.9.11.1) Sublemma. If $J^{\sharp}, K^{\sharp} \subset \mathscr{O}_{X^{\sharp}, P^{\sharp}}$ are ideals generated by semi-invariants and $k \in \mathbb{Z}_{m}$, then

$$
\left(J^{\sharp m} K^{\sharp}\right)_{\{k\}} \subset\left(J_{\{0\}}^{\sharp}\right) \cdot K_{\{k\}}^{\sharp} \quad(c f .(2.5)) .
$$

Indeed for arbitrary semi-invariants $u_{1}, \ldots, u_{m} \in J^{\sharp}$ and $v \in K^{\sharp}$ such that wt $u_{1}+\cdots+$ wt $u_{m}+$ wt $v \equiv k(m)$, there exist $a, b \in[0, m]$ such that $a<b$ and $\sum_{i \leq a}$ wt $u_{i} \equiv \sum_{i \leq b}$ wt $u_{i} \quad(m)$, whence $\sum_{a<i \leq b}$ wt $u_{i} \equiv 0 \quad(m)$. Then (9.9.11.1) follows from

$$
u_{i} \cdots u_{m} v=\left(\prod_{a<i \leq b} u_{i}\right) \cdot\left(\prod_{i \leq a} u_{i} \cdot \prod_{b<i} u_{i} \cdot v\right) \in J_{\{0\}}^{\sharp} \cdot K_{\{k\}}^{\sharp} \cdot
$$

(9.9.12) Case $\ell(P)=1$. By $\ell(P)>0, X^{\sharp}$ is singular and we see that the axial multiplicity $w$ satisfies $w>1$. Then we may rewrite $\phi \equiv y_{1} y_{3}\left(y_{2}, y_{3}, y_{4}\right)^{2}$ as

$$
\left(g_{1} y_{1}+g_{3}\right) y_{3}=y_{2} g_{2}+y_{4}{ }^{w},
$$


where $g_{1}$ is an invariant unit, and $g_{2}, g_{3} \in\left(y_{2}, y_{3}, y_{4}\right) \mathbb{C}\{y\}$ are semi-invariants with $\mathrm{wt} \equiv-a_{2}, a_{1}(m)$. Then $\left(y_{4}, y_{2}, y_{3}\right)$ is a $(1, e, w)$-monomializing (resp. $(1, n e, w)$-monomializing) $\ell$-basis of the second kind for $I \supset J_{e}\left(\right.$ resp. $\left.I \supset J_{n e}\right)$ at $P$ by $(8.11(i i))$. Since $w \mid e$, we have $J_{e}^{\sharp}=\left(y_{2}, y_{3}^{e / w}\right), J_{n e}^{\sharp}=\left(y_{2}, y_{3}^{n e / w}\right)$, and $F^{n e}\left(\mathscr{O}, J_{e}\right)^{\sharp}=\left(y_{2}, y_{3}^{e / w}\right)^{n}$ by $(8.11)$. Hence

$$
J_{n e}^{\sharp} / J^{n e}\left(\mathscr{O}, J_{e}\right)^{\sharp} \simeq \mathscr{O} /\left\{\left(J_{e}^{\sharp}\right)^{n}: y_{2}\right\} \simeq \mathscr{O} /\left(J_{e}^{\sharp}\right)^{n-1}
$$

as in (9.9.11) and the inclusion follows from (9.9.11.1). Thus (9.9.7) is proved.

(9.9.13) For an arbitrary $n \in \mathbb{N}, h$ factors through

$$
h_{n e}: H^{0}\left(\omega_{X}^{*} / F^{n e}\left(\omega_{X}^{*}, J_{e}\right)\right) \rightarrow H^{0}\left(\mathscr{O}_{C}\right)=\mathbb{C}
$$

by construction. By the construction of $J_{n e}$, we see that the image of the natural map $J_{n e} \rightarrow \operatorname{gr}_{C}^{1} \mathscr{O}$ is $\mathscr{M}$ and the composite map

$$
\omega_{X}^{*} \widetilde{\otimes} J_{n e}=F^{n e}\left(\omega_{X}^{*}, J_{n e}\right) \rightarrow \omega_{X}^{*} / F^{n e}\left(\omega_{X}^{*}, J_{e}\right) \rightarrow \omega_{X}^{*} \widetilde{\otimes} \mathscr{M}
$$

is an $\ell$-surjection of $\mathscr{O}_{X}$-modules (cf. (9.9.8)). Thus by (9.9.7), we get the commutative diagram with a surjection $\pi$

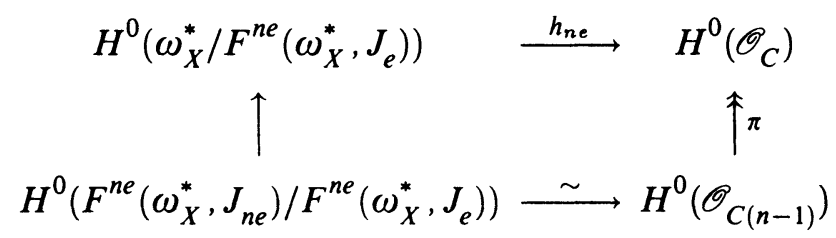

Whence $h_{n e}$ is surjective. Since the topology induced by $\left\{F^{n e}\left(\omega_{X}^{*}, J_{e}\right)\right\}_{n \in \mathbb{N}}$ is equivalent to the usual one (9.9.7), we can apply [Gro, Théorème (4.1.5)] to the total contraction $f: X \rightarrow(Y, Q)$ of $X \supset C$ to get

$$
f_{*} \omega_{X}^{*} \otimes \mathscr{O}_{Y, Q}^{-} \simeq \underset{n}{\lim } H^{0}\left(\omega_{X}^{*} / F^{n e}\left(\omega_{X}^{*}, J_{e}\right)\right)
$$

where $\mathscr{O}_{Y, Q}$ is the completion of $\mathscr{O}_{Y, Q}$. Since $\operatorname{Ker} h_{n e}$ satisfies the MittagLeffler condition, we have a surjection

$$
f_{*} \omega_{X}^{*} \otimes \mathscr{O}_{Y, Q}^{\hat{n}} \rightarrow H^{0}\left(\mathscr{O}_{C}\right) \text {. }
$$

Hence $h$ is a surjection, and (9.9.4) is proved.

Thus by (9.9.3) and (9.9.4), we have the main result of this section.

(9.10) Theorem. Let $X \supset C \simeq \mathbb{P}^{1}$ be an extremal nbd with two points $P, P^{\prime}$ with indices $\geq 3$. Then general members of $\left|-K_{X}\right|$ are normal surfaces containing $C$, smooth outside $\left\{P, P^{\prime}\right\}$, with rational double points of $A$-type at $P, P^{\prime}$. 
Hence the proof of $(0.4 .5)$ is completed and thus the Flip Theorems (0.4.1) and (0.2.5) are proved and the results mentioned in $\S 0$ follow.

\section{A SLight GeNERALIZATION OF (0.4.5) AND COMMENTS ON (1a.7)}

In this section, we state a slight generalization (10.2) of $(0.4 .5)$ and then make comments on (1a.7).

The proof of (10.2) is basically the same as that of (0.4.5). Therefore, we will only indicate changes to be made together with simple explanations.

(10.1) Let $k$ be an algebraically closed field of characteristic 0 . Let $C$ be a connected reduced projective curve over $k$ and let $X \supset C$ be a 3-dimensional normal formal scheme along $C$ which has only terminal singularities. We consider the following condition:

(*) $\quad-K_{X}$ is ample on $C$ and $H^{1}\left(X, \mathscr{O}_{X}(L)\right)=0$ for all Weil divisors $L$ on $X$ such that $n L \sim 0$ or $n K_{X}$ for some $n>0$.

We note that $(*)$ is satisfied by the formal schemes associated to extremal nbds by (1.2.1) and (1.12). The following is the slight generalization of (0.4.5).

(10.2) Proposition. Let $X \supset C$ be as in (10.1) satisfying the condition (*) and such that $C$ is irreducible. Then one of the following on the linear system $\left|-a K_{X}\right|(a=1$, or 2) holds.

(i) $\left|-K_{X}\right|$ has a member $D$ with only rational double points, or

(ii) $\left|-2 K_{X}\right|$ has a member $D$ so that the double cover $Z$ of $X$ with branch locus $D$ has only canonical singularities.

For instance, this applies to a divisorial contraction of a 3-fold contracting an irreducible divisor to a curve.

(10.3) Except for the obvious changes due to the fact that $X \supset C$ is a formal scheme along $C$ rather than the germ of an analytic space along $C$, most arguments use only the condition $(*)$. The only nontrivial changes are the following.

(10.4) Changes in $\S 1$. Let $X \supset C$ be as in (10.1) with the condition $(*)$. Since $H^{1}\left(X, \mathscr{O}_{X}\right)=0(*)$, the description of Pic $X(1.3)$ and $\mathrm{Cl}^{\text {sc }} X$ (1.9) for irreducible $C$ are also proved by the same argument. By $(*)$, the splitting cover $X^{\prime} \supset C^{\prime}(1.12)$ satisfies the condition:

(**) $\quad-K_{X^{\prime}}$ is ample on $C^{\prime}$ and $H^{1}\left(X^{\prime}, \mathscr{O}_{X^{\prime}}\right)=H^{1}\left(X^{\prime}, \omega_{X^{\prime}}\right)=0$.

Since (1.13)-(1.16) need only the property $(* *)$, the results corresponding to (1.13)-(1.16) hold, which are the results needed in later sections.

(10.5) Changes in $\S 1$ b. A "nearby extremal nbd" for a parameter $\lambda$ should be replaced by the generic deformation $X_{\lambda} \supset C_{\lambda}$ over $\overline{k((\lambda))}$ which is to be defined in a similar way. It is easy to see that $X_{\lambda} \supset C_{\lambda}$ satisfies the condition $(*)$. 
(10.6) Changes to be made in $\S \S 6$ and 7. The only place in this paper which uses the property that the total contraction $X \rightarrow Y$ is an isomorphism outside $C$ is $(6.2(\mathrm{i}))$ which asserts that $X$ has index $>1$. Therefore, for our formal scheme $X \supset C \simeq \mathbb{P}^{1}$ with $(*),(6.2(\mathrm{i}))$ should be changed to " $X$ has at most three singular points in $C$ " and thus (6.7) should have an extra case (6.7.0) where $X$ is Gorenstein.

Hence $\S 7$ should treat the extra case (6.7.0), which is very easy: $\left|-K_{X}\right|$ has a smooth member by $(1.3)$ since $\mathscr{O}_{C}\left(-K_{X}\right) \simeq \mathscr{O}(-1)$.

(10.7) It was after this paper had been written that the author learned that [KSB] contained the complete classification of 3-fold terminal singularities [KSB, Theorem 6.5] and settled

(10.8) Theorem (Kollár, Shepherd-Barron). Small deformations of 3-fold terminal singularities are terminal.

(10.9) If we use it, the conditions (1b.3(ii)) and hence (1b.5) became unnecessary, and therefore the arguments for (1b.3), (4.7), (4.12), and (9.6) became simpler but not much. Therefore the revision at this point does not seem worth the trouble, and we decided not to revise the paper.

\section{APPENDIX A. SUMmaRY OF local Classification of $X \supset C \ni P$}

In this appendix, we will summarize the classification of the local structure of an extremal nbd $X \supset C \simeq \mathbb{P}^{1}$ at a singular point $P$.

(A.1) Let $X \supset C \simeq \mathbb{P}^{1}$ be an extremal nbd and $P \in C$ a singular point of $X$ of index $m$. Let $\pi^{\sharp}:\left(X^{\sharp}, P^{\sharp}\right) \rightarrow(X, P)$ be the canonical cover of the germ, and let $C^{\sharp}=\pi^{\sharp-1}(C)_{\text {red }} \subset X^{\sharp}$. We note that $\left(X^{\sharp}, P^{\sharp}\right)$ is at most an isolated $c D V$ singularity (hence embeddable in $\left(\mathbb{C}^{4}, 0\right)$ ) with $\mu_{m}$-action which is free on $X^{\sharp}-P^{\sharp}$ and that $\pi^{\sharp}$ is the $\boldsymbol{\mu}_{m}$-quotient morphism.

There exists a set $(y)=\left(y_{1}, y_{2}, y_{3}, y_{4}\right)$ of $\boldsymbol{\mu}_{m}$-semi-invariant convergent power series giving a $\mu_{m}$-equivariant embedding of $\left(X^{\sharp}, P^{\sharp}\right)$ into $\left(\mathbb{C}^{4}, 0\right)$.

(A.2) Notation. Given a generating character $\chi \in \operatorname{Hom}\left(\boldsymbol{\mu}_{m}, \mathbb{C}^{*}\right) \simeq \mathbb{Z}_{m}$, we write wt $f \equiv a$ for a $\boldsymbol{\mu}_{m}$-semi-invariant $f$ if $g(f)=\chi(g)^{a} f$ for all $g \in \boldsymbol{\mu}_{m}$; $\operatorname{wt}\left(f_{1}, \ldots, f_{r}\right) \equiv\left(a_{1}, \ldots, a_{r}\right)$ for $\boldsymbol{\mu}_{m}$-semi-invariant $f_{1}, \ldots, f_{r}$ if wt $f_{i} \equiv a_{i}$ for all $i$.

(A.3) Summary. By choosing the above $(y)$ and $\chi$ properly, we have the following case-by-case description of $\mathbb{C}^{4} \supset X^{\sharp} \supset C^{\sharp}$, in which $g_{i}$ 's are $\boldsymbol{\mu}_{m}$-semi-invariant convergent power series in $y$ with the specified weights such that $X^{\sharp}$ has at most an isolated $c D V$ point at the origin. Each result is in (4.2)-(4.4) unless otherwise mentioned. 
(IA) There exist positive integers $a_{1}, a_{2}, \alpha, \beta$ such that $\left(a_{1} a_{2}, m\right)=1$, $\alpha a_{1}+\beta a_{2}=m, \operatorname{wt}(y) \equiv\left(a_{1}, a_{2},-a_{1}, 0\right)$,

$$
\begin{gathered}
C^{\sharp}: y_{1}^{a_{2}}-y_{2}^{a_{1}}=y_{3}=y_{4}=0, \\
X^{\sharp}: g_{2} \cdot\left(y_{1}^{a_{2}}-y_{2}^{a_{1}}\right)+g_{3} \cdot y_{3}+g_{4} \cdot y_{4}=0,
\end{gathered}
$$

where $\operatorname{wt}\left(g_{2}, g_{3}, g_{4}\right) \equiv\left(-a_{1} a_{2}, a_{1}, 0\right)$.

(IB) This does not occur by (6.3).

(IC) $\operatorname{wt}(y) \equiv(2, m-2,0,1)$ and $m$ is an odd integer $\geq 5$,

$$
\begin{gathered}
C^{\sharp}: y_{1}^{m-2}-y_{2}{ }^{2}=y_{3}=y_{4}=0, \\
X^{\sharp}: g_{2} \cdot\left(y_{1}^{m-2}-y_{2}^{2}\right)+y_{3}+g_{4} \cdot y_{4}=0,
\end{gathered}
$$

where $\operatorname{wt}\left(g_{2}, g_{4}\right) \equiv(4,-1)$.

In this case, $\left(y_{1}, y_{2}, y_{4}\right)$ induces an embedding $\left(X^{\sharp}, 0\right) \simeq\left(\mathbb{C}^{3}, 0\right)$ in which $C^{\sharp}$ is given by $y_{1}{ }^{m-2}-y_{2}{ }^{2}=y_{4}=0$. The assertion on $\mathrm{wt}(y)$ is a combination of (4.9.(i)), (5.5(i)) and (6.5).

(IIA) $w t(y) \equiv(1,1,3,2), m=4$,

$$
\begin{gathered}
C^{\sharp}: y_{1}-y_{2}=y_{3}=y_{4}=0, \\
X^{\sharp}: g_{2} \cdot\left(y_{1}-y_{2}\right)+g_{3} \cdot y_{3}+g_{4} \cdot y_{4}=0,
\end{gathered}
$$

where $\operatorname{wt}\left(g_{2}, g_{3}, g_{4}\right) \equiv(1,3,0), g_{2}, g_{3} \notin(y)^{2}, g_{4}(0)=0$.

(IIB) $w t(y) \equiv(3,2,1,1), m=4$,

$$
\begin{gathered}
C^{\sharp}: y_{1}{ }^{2}-y_{2}{ }^{3}=y_{3}=y_{4}=0, \\
X^{\sharp}: y_{1}{ }^{2}-y_{2}{ }^{3}+g_{3} \cdot y_{3}+g_{4} \cdot y_{4}=0,
\end{gathered}
$$

where $\operatorname{wt}\left(g_{3}, g_{4}\right) \equiv(1,1), g_{3} \cdot y_{3}+g_{4} \cdot y_{4} \notin(y)^{3}$.

(III) $m=1$,

$$
\begin{gathered}
C^{\sharp}: y_{1}-y_{2}=y_{3}=y_{4}=0, \\
X^{\sharp}: y_{1} \cdot\left(y_{1}-y_{2}\right)+g_{3} \cdot y_{3}+g_{4} \cdot y_{4}=0,
\end{gathered}
$$

where $g_{3}(0)=g_{4}(0)=0$.

This will be proved at the end of (A.3).

$\left(\mathrm{IA}^{\vee}\right)$ There exist positive integers $a_{1}, a_{2}, w_{1}, w_{2}, \alpha, \beta, s, \bar{m}$ such that $s$, $\bar{m}>1, s \bar{m}=m, a_{2} \leq \bar{m} / 2, \alpha a_{1}+\beta a_{2}=\bar{m}, \alpha w_{1}+\beta w_{2} \equiv 0(m), w_{i} \equiv a_{i}$ $(\bar{m})$ for $i=1,2,\left(w_{1} w_{2}, m\right)=1, w \mathfrak{t}(y) \equiv\left(w_{1}, w_{2},-w_{1}, 0\right)$,

$$
\begin{gathered}
C^{\sharp}: y_{1}^{a_{2} s}-y_{2}^{a_{1} s}=y_{3}=y_{4}=0, \\
X^{\sharp}: g_{2} \cdot\left(y_{1}^{a_{2} s}-y_{2}^{a_{1} s}\right)+g_{3} \cdot y_{3}+g_{4} \cdot y_{4}=0,
\end{gathered}
$$

where $\operatorname{wt}\left(g_{2}, g_{3}, g_{4}\right) \equiv\left(-s a_{1} a_{2}, a_{1}, 0\right)$; and $\partial g_{3} / \partial y_{1}(0) \neq 0$ or $g_{4}=1$. 
$\left(I^{\vee}\right)$ This does not occur by $(6.1)$.

$\left(\right.$ II $\left.^{\vee}\right) \operatorname{wt}(y) \equiv(1,3,3,2), m=4$,

$$
\begin{gathered}
C^{\sharp}: y_{1}{ }^{2}-y_{2}{ }^{2}=y_{3}=y_{4}=0, \\
X^{\sharp}: y_{1}{ }^{2}-y_{2}{ }^{2}+g_{3} \cdot y_{3}+g_{4} \cdot y_{4}=0,
\end{gathered}
$$

where $\operatorname{wt}\left(g_{3}, g_{4}\right) \equiv(3,0)$ and $g_{4}(0)=0$.

We note that the $\boldsymbol{\mu}_{m}$-action on $X^{\sharp}-\{0\}$ is free in all the above cases by the smoothness of $X^{\sharp}-\{0\}$.

Proof for case (III). The assertion on the equation of $X^{\sharp}$ is equivalent to $i_{P}(1)$ $=1\left(2.16(\right.$ ii) $)$. If $i_{P}(1)>1$, then by a separating $L$-deformation (4.7) one obtains an extremal nbd $X^{\prime} \supset C^{\prime}$ with two ordinary double points $Q, R$ on $C^{\prime}$. Since $w_{Q}^{*}(1)=w_{R}^{*}(1)=1(4.9(\mathrm{ii})), X^{\prime}$ has another singular point on $C^{\prime}$ by $\sum_{x \in C^{\prime}} w_{x}^{*}(1) \leq 1$ (2.3.3). It contradicts $(6.2(\mathrm{ii}))$.

(A.4) Remarks. (i) For the simplicity of the formulation, $(y)$ above is different from $(x)$ which is used in (4.2). Our $y_{i}$ is $x_{i}$ in (4.2) for $i=1,2$ and it is $F_{i}$ in (4.3) for $i=3,4$,

(ii) we note that $\left(X^{\sharp}, 0\right)$ is smooth or a $c A$ type point (1a.2), except in the case (IA),

(iii) when $X \supset C$ is as in (10.2) and not an extremal nbd, we still have the same local results (cf. $\S 10$, especially (10.6)), and

(iv) we note that $a_{1}, a_{2}$ in ( $\left.\mathrm{IA}^{\vee}\right)$ need not be prime to $m$, as in an example of $X \supset C \ni P$ for which $a_{1}=3, a_{2}=2, w_{1}=3+41 \cdot 2=85, w_{2}=2+41 \cdot 3=$ $125, \alpha=13, \beta=1, s=6, \bar{m}=41, m=41 \cdot 6=246$.

\section{APPendiX B. Possible singularities on AN EXTREMAL NBD}

In this appendix, we make a list of possible singularities of an extremal nbd as a summary.

(B.1) Summary. Let $X \supset C \simeq \mathbb{P}^{1}$ be an extremal nbd. The following is the list of all the possible singularities of $X$ on $C$.

Case (1) (treated in (6.7.1) and (7.3.1)):

(a) a type (IA) point $P$ and at most one type (III) point $Q$,

(b) a type (IIA) point $P$ and at most one type (III) point $Q$,

(c) a type (IA ${ }^{\vee}$ ) point $P$ and at most one type (III) point $Q$,

(d) a type (II ${ }^{\vee}$ ) point $P$ (cf. (6.1(iii))).

In (1), a general member $D \in\left|-K_{X}\right|$ satisfies $D \cap C=\{P\}$ (7.3) and has only rational double points as singularities.

Case (2) (treated in (6.7.2) and (7.3.2)):

(e) a type (IC) point $P$, or

(f) a type (IIB) point $P$. 
Case (3) (treated in (6.7.3) and (7.3.3)):

(g) two type (IA) points $P, Q$ and at most one type (III) point $R$ such that index $Q=2$ and $P$ is a type $c A$ point of index $\geq 3$ and size $=1$ (cf. (7.3)).

In (2) and (3), a general member $E \in\left|-2 K_{X}\right|$ is such that $E \cap C=\{P\}$ and such that the double cover of $X$, branched along $E$ and at $Q$, has only canonical singularities.

Case (4) (treated in (6.7.4) and in $\S 9$ ):

(h) two type (IA) points $P, Q$ with indices $\geq 3$.

In (4), a general member $D \in\left|-K_{X}\right|$ contains $C$, is smooth outside of $\{P, Q\}$, and has rational double points of type $A$ at $P$ and $Q(9.10)$.

(B.2) Remark. When $X \supset C$ is as in (10.2) and not an extremal nbd, the results are the same except that we have an extra case:

Case (5) (this is not an extremal nbd by (6.2(i))):

(i) at most one type (III) point $P$.

In this case $\left(-K_{X} \cdot C\right)=1, \mathscr{O}\left(-K_{X}\right)$ is generated by global sections, and $\left|-K_{X}\right|$ has a smooth member (10.6).

This is the Gorenstein case as mentioned at (10.6) and the same argument as in the proof of (III) of (A.3) shows that $X$ has at most one type (III) singular point on $C$.

\section{LIST OF NOTATION AND TERMINOLOGY}

Numbers on the right show the paragraphs where the notations/symbols are defined/explained.

[H]

(1.12), (1a.1), (1b.1), (2.4), (9.1)

[b] b

[(] $(1, d)$-monomializable

$(1, d)$-monomializing $\ell$-basis

$(1, d, b)$-monomializable at $P$

$(1, d, b)$-monomializing $\ell$-basis of the first kind

$(1, d, b)$-monomializing $\ell$-basis of the second kind

$\left(C^{\sharp}, P^{\sharp}\right)$

(2.4), (9.1)

$\left(C^{b}, P^{b}\right)$

$\left(C^{\dagger}, P^{\dagger}\right)$

(I), (IA), (IB), (IC)

(II), (IIA), (IIB)

(III)

$\left(I^{\vee}\right),\left(I A^{\vee}\right),\left(I C^{\vee}\right)$

(II ${ }^{\vee}$ )

[0] 0-sequence

Conventions

[A] $a_{i}$

(2.6), (4.2), (9.1) 


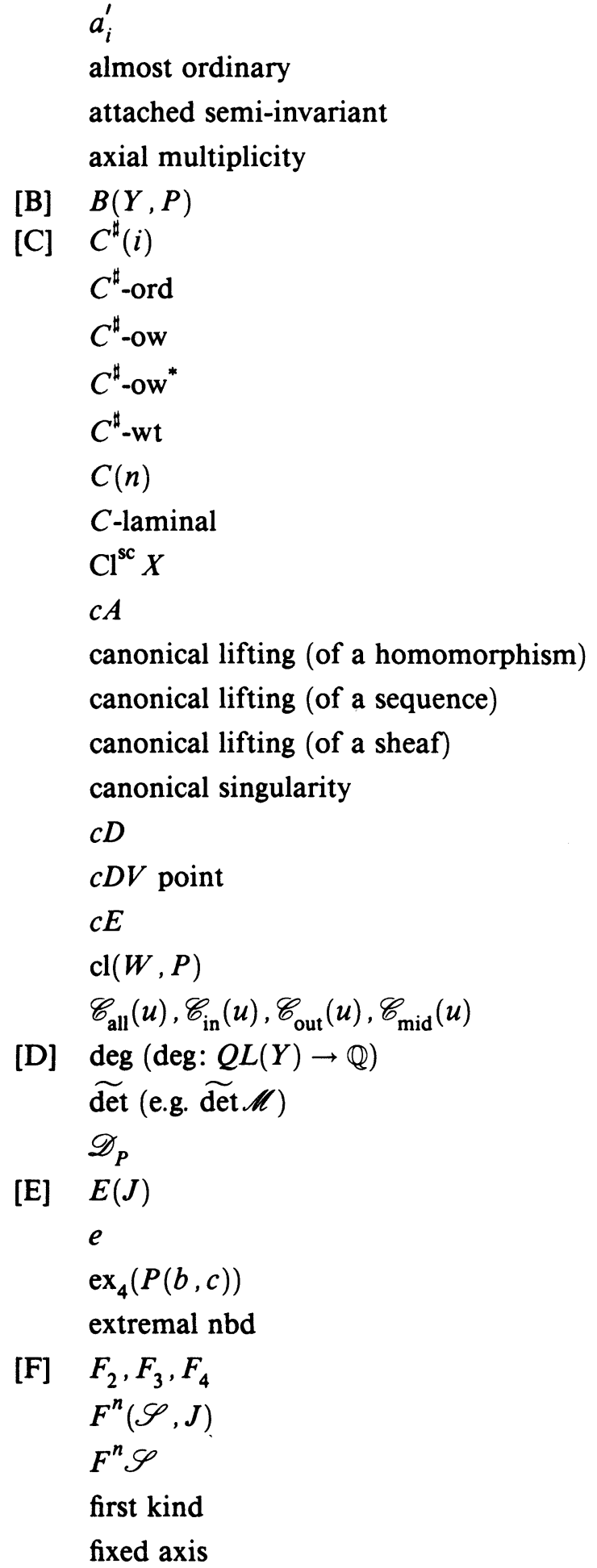

[D] $\operatorname{deg}(\operatorname{deg}: Q L(Y) \rightarrow \mathbb{Q})$

$\widetilde{\operatorname{det}}($ e.g. $\widetilde{\operatorname{det}} \mathscr{M})$

$\mathscr{D}_{P}$

[E] $E(J)$

$e$

$\operatorname{ex}_{4}(P(b, c))$

extremal nbd

[F] $F_{2}, F_{3}, F_{4}$

$F^{n}(\mathscr{S}, J)$

$F^{n} \mathscr{S}$

first kind

fixed axis 
[G] $\operatorname{gr} \mathscr{O}\left(=\bigoplus_{n \geq 0} \operatorname{gr}_{C}^{n} \mathscr{O}\right)$

$\operatorname{gr}(\mathscr{S}, J)$

$\operatorname{gr}^{n}(\mathscr{S}, J)$

$\mathrm{gr}^{n, i}(\mathscr{S}, J)$

$\mathrm{gr}_{C}=\bigoplus_{n \geq 0} \mathrm{gr}_{C}^{n}$

$\mathrm{gr}_{C}^{n}$

[H] $h: H^{0}\left(\omega_{X}^{*}\right) \rightarrow H^{0}\left(\mathscr{O}_{C}\right)=\mathbb{C}$

[I] $I^{\sharp}, I^{\mathrm{b}}$

$i_{P}(n)$

imprimitive

index

induced $\ell$ - structure

[J] $J_{n}$

$J$-filtration

[K] $\mathscr{K}_{n}, \mathscr{K}_{n}^{\prime}, \mathscr{K}_{n}^{\circ}$

[L] $\quad L^{\prime}$-deformation

$L$-deformation

$L$-smoothing

$L G$-deformation

laminal

$l$ deg $\left(\right.$ e.g. $\left.l \operatorname{deg}_{Y} \mathscr{M}\right)$

lif

locally a nested c.i.

locally $\ell$-free

locally primitive

locally primitive at $P$

locally $q \ell$-free

$\mathscr{L}$

$\ell($ e.g. $\ell(P))$

$\ell$-basis

$\ell$-character

$\ell$-coherent

$\ell$-coordinate

$\ell$-coordinate system 
$\ell$-equation

(1a.5)

$\ell$-exact

(8.8.2), (8.9)

$\ell$-free $\ell$-basis at $P$

$\ell$-free $\mathscr{O}_{Y}$-module at $P$

$\ell$-homomorphism

(8.8.2), (8.9)

$\ell$-injection

$(8.8 .2),(8.9)$

$\ell$-invertible

(8.8.3), (8.9)

$\ell$-isomorphism

(8.8.2), (8.9)

$\ell$-split

(8.8.2), (8.9)

$\ell$-splitting

(8.8.2), (8.9)

$\ell$-structure at $P$

$\ell$-surjection

(8.8.2), (8.9)

$\ell$-trivial

[M] minimal

(8.10), (8.11)

monomializable

(8.10), (8.11)

monomializing

9.8.3.1), (9.9)

[N] nearby extremal nbd

nested c.i.

nested complete intersection

normalized expression

normalized $\ell$-coordinates

$\mathbb{N}$

Conventions

[O] ord

(2.5), (9.1)

ord $^{\prime}$

$\operatorname{ord}\left(C^{\sharp}\right)$

ordered ( $\ell$-basis)

(8.10), (8.11)

ordinary

ow

(2.5), (9.1)

$\mathrm{ow}_{i}$

ow'

ow $\left(C^{\sharp}\right)$

ow ${ }^{*}$

[P] $P(b, c)$

primitive point 
pure of rank

pure width

[Q] $Q L($ e.g. $Q L(Y))$

$q$ (e.g. $q(P))$

$q l$ (e.g. $\left.q l_{Y}(\mathscr{M})\right)$

$q l \operatorname{deg}(\mathscr{M}, P)$

$q \ell$-free

$q \ell$-invertible

(8.8.3), (8.9)

quo

(8.8.1)

$\mathbb{Q}$

[R] $R$

$R^{\prime}$

Conventions

rational singularity

(2.8), (9.1)

reduced

res

[S] $\quad S(d)$

$\operatorname{SSQ}(\mathscr{S}), \operatorname{SSQ}\left(\mathscr{S}, \mu_{m}\right)$

$\tilde{S}$ (e.g. $\left.\tilde{S}^{n}(\mathscr{M})\right)$

saturated submodule

saturated chain

saturated subquotient

saturation of submodule

saturation of chain

second kind

semi-Cartier divisor class group

separating $L$-deformation

size, $\operatorname{siz}_{P}$

splitting cover

splitting degree

subindex

subquotient

$\mathfrak{S}_{n}$ (symmetric group of degree $n$ )

[T] $\quad T L(T L: Q L(Y) \rightarrow \mathbb{Z})$

$$
t
$$

$t^{\prime}$ 
terminal singularity

total contraction

twisted extension

[U] $U$

$U^{\prime}$

$u_{1}, u_{1}^{\prime}$

$u_{2}, u_{2}^{\prime}$

$\mathscr{U}\left(\begin{array}{c}I \times \delta \times \delta \\ u\end{array}\right)$

The paragraph preceding (9.3.2)

[W] $w, w^{\prime}$

$w_{P}(n)$

$w_{P}^{*}(n)=\left(\begin{array}{c}n+1 \\ 2\end{array}\right) i_{P}(1)-w_{P}(n)$

width (of an element), width ${ }_{J} S$

width (of a laminal ideal)

wt

$\mathrm{wt}^{\prime}$

[X] $X_{\lambda, \text { in }}, X_{\lambda, \text { mid }}, X_{\lambda, \text { out }}$

$X\left(\begin{array}{l}I \times \gamma \\ v<\delta\end{array}\right)$

$X[D, d, \phi]$

$x=\left(x_{1}, \ldots, x_{4}\right)$

$x^{\prime}=\left(x_{1}^{\prime}, \ldots, x_{4}^{\prime}\right)$

$\mathscr{X}_{\text {all }}(u), \mathscr{X}_{\text {in }}(u), \mathscr{X}_{\text {mid }}(u), \mathscr{X}_{\text {out }}(u)$

$\mathscr{X}\left(\begin{array}{c}I \times \gamma \times \delta \\ u<\delta\end{array}\right)$

[Z] $\mathbb{Z}_{+}, \mathbb{Z}_{++}, \mathbb{Z}_{m}$

Conventions

[ [] 「 1 (round up)

Conventions

[ [ ] [ ] (Gaussian symbol)

Conventions

$[\cdot, \cdot, \cdot]$

[n] (e.g. $M[n]$ for $n \in \mathbb{Z}_{m}$ )

Rem : $M[n]$ is defined in two places in equivalent ways.

[ \{]$_{\{n\}}\left(\right.$ e.g. $\left.M_{\{n\}}\right)$

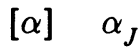

$\alpha_{n}$

$\alpha_{n}: \operatorname{gr}_{C}^{0} J_{n} \rightarrow \mathscr{M}$

Rem : The above two $\alpha_{n}$ 's have nothing to do with each other. 
$[\beta] \quad \beta_{J}$

$\beta_{n}$

$[\gamma] \quad \gamma^{n, i}(\mathscr{S}, J)$

$\gamma^{n}(\mathscr{S}, J)$

$\tilde{\gamma}^{n, i}(\mathscr{S}, J)$

(8.10), (8.11.1), (8.12)

$\tilde{\gamma}^{n}(\mathscr{S}, J)$

(8.10), (8.12)

$\gamma_{C}^{n}$

[ $\Delta] \quad \Delta_{J}$

[ $\delta] \quad \delta_{i, j}($ Kronecker's $\delta)$

Conventions

$\delta_{J^{\sharp}, P^{\sharp}}^{\cdot}, \delta_{J^{\sharp}}^{n}$

$[\theta] \quad \theta(n)$

[ $\pi] \quad \pi^{\sharp}:\left(X^{\sharp}, P^{\sharp}\right) \rightarrow(X, P), \pi^{b}:\left(X^{b}, P^{b}\right) \rightarrow\left(X, P^{\prime}\right)$

$\pi[D, d, \phi]$

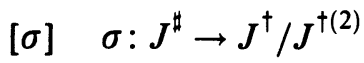

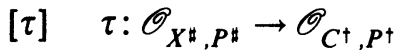

[Ф] $\Phi^{i} \operatorname{gr}^{n}(\mathscr{S}, J)$

[ $\mu$ ] $\boldsymbol{\mu}_{m}$

$\boldsymbol{\mu}_{d}$-cover associated to $[D, \phi]$

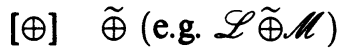

$[\otimes] \quad \tilde{\otimes},{ }^{\otimes}\left(\right.$ e.g. $\left.\mathscr{L} \widetilde{\otimes} \mathscr{M}, \mathscr{L}^{\widetilde{\otimes} n}\right)$

[ | ] \| \|

\section{REFERENCES}

[A1] M. Artin, On the solutions of analytic equations, Invent. Math. 5 (1968), 277-291.

[A2] _ Algebraic approximation of structures over complete local rings, Inst. Hautes Études Sci. Publ. Math. 36 (1969), 23-58.

[B1] X. Benveniste, Sur l'anneau canonique de certaines variétés de dimension 3, Invent. Math. 73 (1983), 157-164.

[B2] __, Sur le cone des 1-cycles effectifs en dimension 3, Math. Ann. 272 (1985), 257-265.

[BS] J. Bingener and U. Storch, Zur Berechnung der Divisorenklassengruppen kompletter lokaler Ringe, Nova Acta Leopoldina (N.F.) 52 Nr. 240 (1981), 7-63.

[Da] V. I. Danilov, Birational geometry of toric 3-folds, Math. USSR-Izv. 21 (1983), 269-279.

[E11] R. Elkik, Singularités rationnelles et déformations, Invent. Math. 47 (1978), 139-147.

[E12] __, Rationalité des singularités canoniques, Invent. Math. 64 (1981), 1-6.

[Fl] H. Flenner, Rational singularities, Arch. Math. 36 (1981), 35-44.

[Fr] P. Francia, Some remarks on minimal models. I, Compositio Math. 40 (1980), 301-313.

[Ft] T. Fujita, Zariski decomposition and canonical rings of elliptic threefolds, J. Math. Soc. Japan 38 (1986), 20-37. 
[Go] R. Godement, Topologie algébrique et théorie des faisceaux, Hermann, Paris, 1958.

[Gra] H. Grauert, On Levi's problem and the embedding of real-analytic manifolds, Ann. of Math. 68 (1958), 460-472.

[GR] H. Grauert and O. Riemenshneider, Verschwindungssätze für analytische Kohomologiegruppen auf Komplexen Raumen, Invent. Math. 11 (1970), 263-292.

[Gro] A. Grothendieck, Eléments de géométrie algébrique. III, Inst. Hautes Études Sci. Publ. Math. 20 (1964).

[H] H. Hironaka, On the theory of birational blowing-up, Thesis, Harvard Univ., 1960.

[I] V. A. Iskovskih, Minimal models of rational surfaces over arbitrary fields, Math. USSR-Izv. 14 (1980), 17-39.

[Ka1] Y. Kawamata, On the finiteness of generators of a pluricanonical ring for a 3-fold of general type, Amer. J. Math. 106 (1984), 1503-1512.

[Ka2] _ , The crepant blowing-ups of 3-dimensional canonical singularities and its application to degenerations of surfaces, Ann. of Math. (to appear).

[KMM] Y. Kawamata, K. Matsuda, and K. Matsuki, Introduction to the minimal model problem, Proc. Sympos. Algebraic Geom., Sendai, 1985, Adv. in Pure Math. 10 (1987), 283-360.

[KI] S. Kleiman, Toward a numerical theory of ampleness, Ann. of Math. 84 (1966), 293-344.

[Ko1] J. Kollár, Higher direct images of dualizing sheaves, Ann. of Math. 123 (1986), 11-42.

[Ko2] _ , The structure of algebraic threefolds-an introduction to Mori's program, Bull. Amer. Math. Soc. 17 (1987), 215-277.

[Ko3] _ Flops, Nagoya Math. J. (to appear).

[KSB] J. Kollár and N. Shepherd-Barron, Threefolds and deformation of surface singularities, Preprint.

[Ku] V. Kulikov, Degenerations of K3 surfaces and Enriques surfaces, Math. USSR-Izv. 11 (1977), 957-989.

[Ma] Yu. I. Manin, Rational surfaces over perfect fields, Inst. Hautes Études Sci. Publ. Math. 30 (1966), 55-114.

[Mn] M. Miyanishi, Projective degeneration of surfaces according to S. Tsunoda, Proc. Sympos. Algebraic Geom., Sendai, 1985, Adv. in Pure Math. 10 (1987), 415-447.

[My1] Y. Miyaoka, Deformations of a morphism along a foliation and a birational criterion of conic bundles, Proc. Sympos. Pure Math. (Summer Research Inst., Bowdoin, 1985), Vol. 46 (1987), pp. 245-268.

[My2] _ , The Chern classes and Kodaira dimension of a minimal variety, Proc. Sympos. Algebraic Geom., Sendai, 1985, Adv. in Pure Math. 10 (1987), 449-476.

[My3] _ On the Kodaira dimension of Minimal Threefolds, Preprint.

[MM] Y. Miyaoka and S. Mori, A numerical criterion of uniruledness, Ann. of Math. 124 (1986), 65-69.

[Mr1] S. Mori, Projective manifolds with ample tangent bundles, Ann. of Math. 110 (1979), 593-606.

[Mr2] _ Threefolds whose canonical bundles are not numerically effective, Ann. of Math. 116 (1982), 133-176.

[Mr3] _, On 3-dimensional terminal singularities, Nagoya Math. J. 98 (1985), 43-66.

[Mr4] _ Classification of higher dimensional varieties, Proc. Sympos. Pure Math. (Summer Research Inst., Bowdoin, 1985), Vol. 46 (1987), pp. 269-331.

[Mrr1] D. Morrison, Semistable degenerations of Enriques and hyperelliptic surfaces, Duke Math. J. 48 (1981), 197-249.

[Mrr2] _ A remark on Kawamata's paper "On the plurigenera of minimal algebraic 3-folds with $K \approx 0$ ", Math. Ann. 275 (1986), 547-553.

[MS] D. Morrison and G. Stevens, Terminal quotient singularities in dimension 3 and 4 , Proc. Amer. Math. Soc. 90 (1984), 15-20. 
[N] N. Nakayama, Invariance of the plurigenera of algebraic varieties under minimal model conjectures, Topology 25 (1986), 237-251.

[PP] U. Persson and H. Pinkham, Degeneration of surfaces with trivial canonical divisor, Ann. of Math. 113 (1981), 45-66.

[R1] M. Reid, Minimal models of canonical 3-folds, Algebraic Varieties and Analytic Varieties (S. Iitaka, ed.), Advanced Studies in Pure Math. 1 (1983), Kinokuniya, Tokyo, and NorthHolland, Amsterdam, pp. 131-180.

[R2] _ Decomposition of toric morphisms, Arithmetic and geometry. II, Progress in Math. 36 (1983), 395-418.

[R3] _ _ Tendencious survey of 3-folds, Proc. Sympos. Pure Math. (Summer Research Inst., Bowdoin, 1985), Vol. 46 (1987), 333-344.

[R4] _ Young person's guide to canonical singularities, Proc. Sympos. Pure Math. (Summer Research Inst., Bowdoin, 1985), Vol. 46 (1987), pp. 345-414.

[SB] N. Shepherd-Barron, Some questions on singularities in 2 and 3 dimensions, Thesis, Univ. of Warwick, 1980.

[Sh] V. V. Shokurov, The non-vanishing theorem, Izv. Akad. Nauk. SSSR Ser. Mat. 49 (1985), 635-651; Math. USSR-Izv. 26 (1986), 591-604.

[T] S. Tsunoda, Degenerations of surfaces, Proc. Sympos. Algebraic Geom., Sendai, 1985, Adv. in Pure Math. 10 (1987), 755-764.

[Wa] J. Wahl, Equisingular deformations of normal surface singularities, Ann. of Math. 104 (1976), 325-356.

[Wi] P. M. H. Wilson, Towards birational classification of algebraic varieties, Bull. London Math. Soc. 19 (1987), 1-48.

Department of Mathematics, Nagoya University, Nagoya 464 Japan (Current address)

Department of Mathematics, Columbia University, New York, New York 10027 